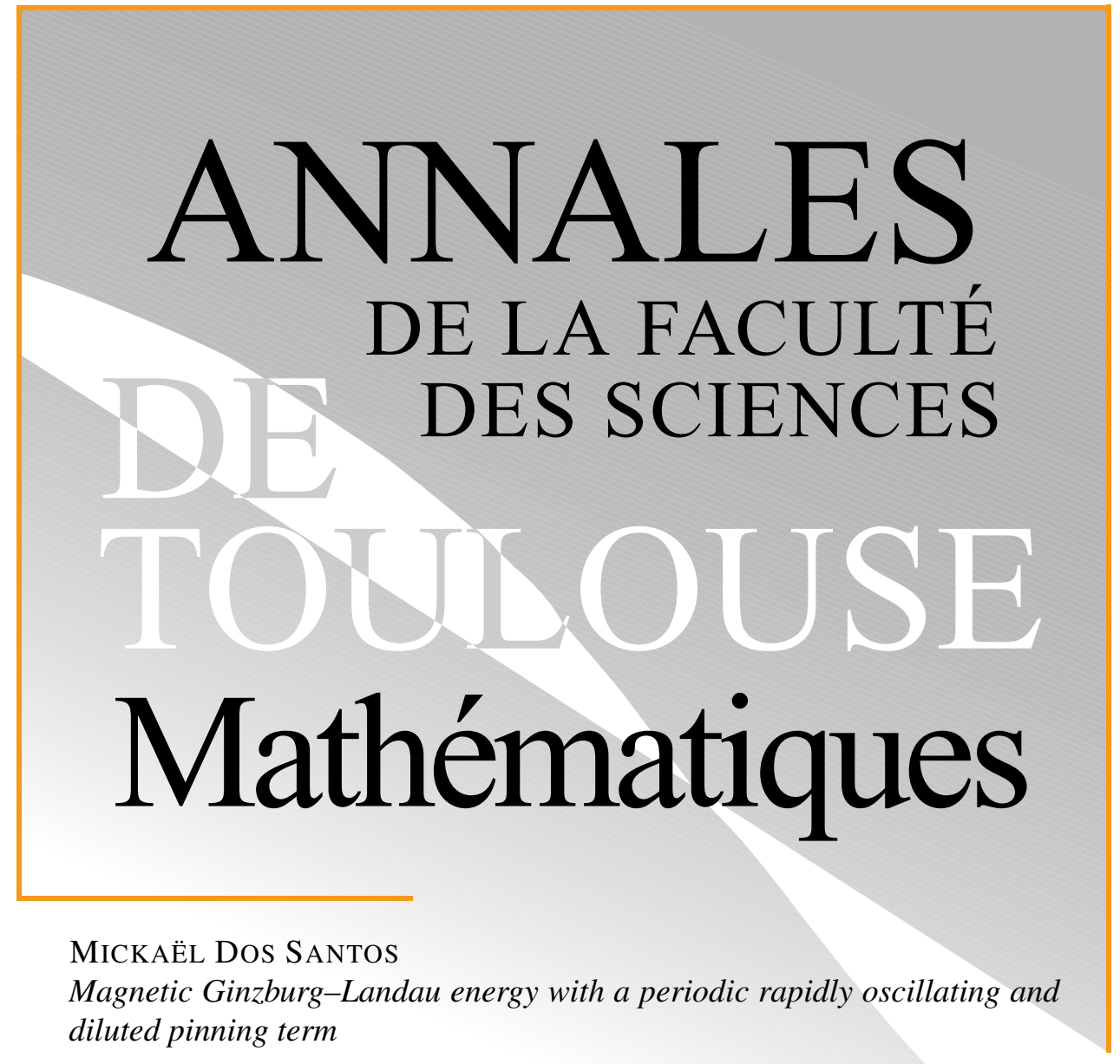

Tome XXX, no 4 (2021), p. 705-799.

https://doi.org/10.5802/afst.1688

(C) Université Paul Sabatier, Toulouse, 2021.

L'accès aux articles de la revue «Annales de la faculté des sciences de Toulouse Mathématiques » (http://afst.centre-mersenne.org/) implique l'accord avec les conditions générales d'utilisation (http://afst.centre-mersenne.org/legal/). Les articles sont publiés sous la license CC-BY 4.0.

(c)

Publication membre du centre

Mersenne pour l'édition scientifique ouverte MERSENNE http://www.centre-mersenne.org/ 


\title{
Magnetic Ginzburg-Landau energy with a periodic rapidly oscillating and diluted pinning term ${ }^{(*)}$
}

\author{
MiCKAËL DOS SANTOS ${ }^{(1)}$
}

\begin{abstract}
We study the $2 D$ full Ginzburg-Landau energy with a periodic rapidly oscillating, discontinuous and (strongly) diluted pinning term using a perturbative argument. This energy models the state of an heterogeneous type II superconductor submitted to a magnetic field. We calculate the value of the first critical field which links the presence of vorticity defects with the intensity of the applied magnetic field. Then we prove a standard dependence of the quantized vorticity defects with the intensity of the applied field. Our study includes the case of a London solution having several minima. The pinning effect is explicitly established and we give the asymptotic location of the vorticity defects with various scales. The macroscopic location of the vorticity defects is understood with the famous BethuelBrézis-Hélein renormalized energy restricted to the minima of the London solution coupled with a renormalized energy obtained by Sandier-Serfaty. The mesoscopic location, i.e., the arrangement of the vorticity defects around the minima of the London solution, is described, as in the homogenous case, by a renormalized energy obtained by Sandier-Serfaty. The microscopic location is exactly the same than in the heterogeneous case without magnetic field. We also compute the value of secondary critical fields that increment the quantized vorticity.
\end{abstract}

RÉsumé. - À l'aide d'un argument perturbatif, on étudie une énergie de type Ginzburg-Landau bidimensionnelle avec un champ magnétique et présentant un terme de chevillage périodique rapidement oscillant, discontinu et (fortement) dilué. Cette énergie modélise l'état d'un supraconducteur hétérogène de type II soumis à un champ magnétique. On calcule la valeur du premier champ critique à partir duquel les défauts de vorticité apparaissent. Ensuite on démontre une dépendance classique reliant les défauts de vorticité quantifiés avec l'intensité du champ appliqué. Notre étude traite aussi le cas où la solution de London admet plusieurs point de minimum. L'effet d'ancrage des défauts de vorticité est clairement établi et on précise suivant différentes échelles l'emplacement asymptotique des défauts de vorticité. La position macroscopique des défauts de vorticité est donnée par la célèbre énergie renormalisée de Bethuel-Brézis-Hélein restreinte au points de minimum de la solution

$\left.{ }^{*}\right)$ Reçu le 8 avril 2019, accepté le 20 octobre 2019.

Keywords: Superconductivity, Ginzburg-Landau, pinning.

2010 Mathematics Subject Classification: 35Q56, 35J20, 35B27.

(1) Laboratoire de Mathématiques Blaise Pascal, Université Clermont Auvergne, UMR CNRS 6620, Campus des Cézeaux, 63177 Aubière, France mickael.dos_santos@uca.fr

Article proposé par Radu Ignat. 
de London couplée avec une énergie renormalisée obtenue par Sandier-Serfaty. La position mesoscopique, i.e., l'arrangement des défauts de vorticité autour des points de minimum de la solution de London, est décrite, comme dans le cas homogène, par une énergie renormalisée obtenue par Sandier-Serfaty. La position microscopique est exactement la même que dans le cas sans champ magnétique. On calcule aussi des champs critiques secondaires qui incrémentent la vorticité quantifiée.

\section{Introduction}

This article studies the pinning phenomenon in type-II superconducting composites.

Superconductivity is a property that appears in certain materials cooled below a critical temperature. These materials are called superconductors. Superconductivity is characterized by a total absence of electrical resistance and a perfect diamagnetism. Unfortunately, when the imposed conditions are too intense, superconductivity is destroyed in certain areas of the material called vorticity defects.

We are interested in type II superconductors which are characterized by the fact that the vorticity defects first appear in small areas. Their number increases with the intensity of the conditions imposed until filling the material. For example, when the intensity $h_{\mathrm{ex}}$ of an applied magnetic field exceeds a first threshold, the first vorticity defects appear: the magnetic field begins to penetrate the superconductor. The penetration is done along thin wires and may move resulting an energy dissipation. These motions may be limited by trapping the vorticity defects in small areas.

The behavior of a superconductor is modeled by minimizers of a Ginzburg-Landau type energy. In order to study the presence of traps for the vorticity defects we consider an energy including a pinning term that models impurities in the superconductor. These impurities would play the role of traps for the vorticity defects. We are thus lead to the subject of this article: the type-II superconducting composites with impurities.

The case of an infinite long homogenous type II superconducting cylinder was intensively studied in mathematics by various authors since the 90's (see [16] for a guide to the litterature). Namely, the present work deals with a cylindrical superconductor $\mathcal{S}=\Omega \times \mathbb{R}$ (whose section is $\Omega \subset \mathbb{R}^{2}$ ) submitted to a vertical magnetic field $\left(0,0, h_{\mathrm{ex}}\right)$. Under these considerations, the vorticity defects are thin vertical cylinders. Thus their study may be done via a $2 \mathrm{D}$ problem formulated on $\Omega \subset \mathbb{R}^{2}$. Following the works of various authors (see $[1,11,14])$, for a small parameter $\varepsilon>0(\varepsilon \rightarrow 0$ in this article) 
and $h_{\mathrm{ex}}=h_{\mathrm{ex}}(\varepsilon) \geqslant 0$, we are interested in the description of the (global) minimizers of the functional

$$
\begin{aligned}
\mathcal{E}_{\varepsilon, h_{\mathrm{ex}}}: \quad \mathscr{H} & \longrightarrow \mathbb{R}^{+} \\
(u, A) & \longmapsto \frac{1}{2} \int_{\Omega}|\nabla u-\imath A u|^{2}+\frac{1}{2 \varepsilon^{2}}\left(a_{\varepsilon}^{2}-|u|^{2}\right)^{2}+\left|\operatorname{curl}(A)-h_{\mathrm{ex}}\right|^{2}
\end{aligned}
$$

where (see Section 2 for more detailed notation)

- $\Omega \subset \mathbb{R}^{2}$ is a smooth bounded simply connected open set,

- $\mathscr{H}:=H^{1}(\Omega, \mathbb{C}) \times H^{1}\left(\Omega, \mathbb{R}^{2}\right)$,

- $a_{\varepsilon}: \Omega \rightarrow\{1, b\}(b \in(0,1)$ is independent of $\varepsilon)$ is a periodic diluted pinning term (see Figure 1.1 and Section 2.3 for a construction of $\left.a_{\varepsilon}\right)$. The impurities are the connected components of $\omega_{\varepsilon}:=a_{\varepsilon}^{-1}(\{b\})$. In the definition of $a_{\varepsilon}, \delta=\delta(\varepsilon) \underset{\varepsilon \rightarrow 0}{\rightarrow} 0$ is the parameter of period, $\lambda=\lambda(\varepsilon) \underset{\varepsilon \rightarrow 0}{\rightarrow} 0$ is the parameter of dilution and $0 \in \omega \subset \mathbb{R}^{2}$ is a smooth bounded simply connected open set which gives the form of the impurities.

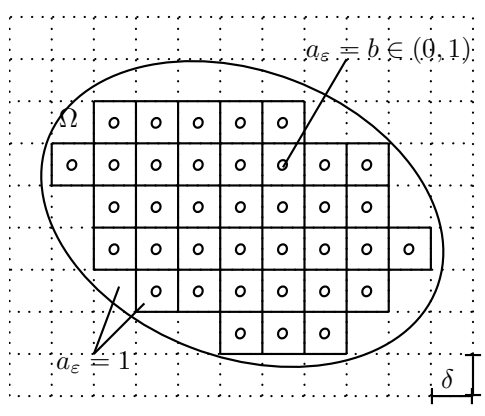

(a) The pining term is periodic on a $\delta \times \delta$-grid

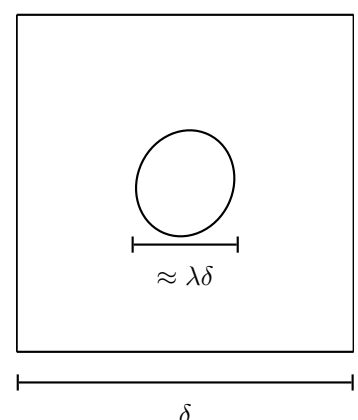

(b) The parameter $\lambda$ controls the size of an inclusion in the cell

Figure 1.1. The periodic pinning term

We focus on a strongly diluted case $\lambda^{1 / 4}|\ln \varepsilon| \rightarrow 0$ with not too small connected components of $\omega_{\varepsilon}$ in order to trap the vorticity defects $|\ln (\lambda \delta)|=$ $\mathcal{O}(\ln |\ln \varepsilon|)$ but with a sufficiently small parameter of the period (see (1.4)).

Under these considerations, if $\left(u_{\varepsilon}, A_{\varepsilon}\right)$ minimizes $\mathcal{E}_{\varepsilon, h_{\mathrm{ex}}}$, then the vorticity defects may be interpreted as the set $\left\{\left|u_{\varepsilon}\right|<b / 2\right\}$. It is excepted that the connected components of $\left\{\left|u_{\varepsilon}\right|<b / 2\right\}$ are close to disks with radii of order $\varepsilon$. 
As said above, our study takes place in the extrem type II case $\varepsilon \rightarrow 0$ and we also assume a divergent upper bound for $h_{\mathrm{ex}}$. Vorticity defects appear for minimizers above a critical valued

$$
H_{c_{1}}=\left[b^{2}|\ln \varepsilon|+\left(1-b^{2}\right)|\ln (\lambda \delta)|\right] /\left(2\left\|\xi_{0}\right\|_{L^{\infty}(\Omega)}\right)+\mathcal{O}(1)
$$

(see Corollary 10.3 and (7.5)). Here $\xi_{0} \in H_{0}^{1} \cap H^{2}$ is called the London solution and is the unique solution of the London equation

$$
\begin{cases}-\Delta^{2} \xi_{0}+\Delta \xi_{0}=0 & \text { in } \Omega \\ \Delta \xi_{0}=1 & \text { on } \partial \Omega \\ \xi_{0}=0 & \text { on } \partial \Omega .\end{cases}
$$

The value $H_{c_{1}}$ is calculated by a standard balance of the energetic costs of a configuration without vorticity defects $|u| \geqslant b / 2$ with well prepared competitors having an arbitrary number of quantized vorticity defects. Here quantization has to be interpreted by the degree of $u$ around a vorticity defect. It is an observable quantity related with the circulation of the superconducting currents.

In order to lead the study, the set $\Lambda:=\left\{z \in \Omega \mid \xi_{0}(z)=\min \xi_{0}\right\} \subset \Omega$ is of major interest (it is standard to prove that, in $\Omega,-1<\xi_{0}<0$ ). From Lemma 4.4 in [17] and Lemma 4 in [15] we have the following:

Lemma 1.1. - The set $\Lambda$ is finite. Moreover there exist $\eta>0$ and $M \geqslant 1$ s.t. for $a \in \Omega$ we have $\xi_{0}(a) \geqslant \min \xi_{0}+\eta \operatorname{dist}(a, \Lambda)^{M}$. $^{(1)}$

We write $N_{0}:=\operatorname{Card}(\Lambda)$ and $\Lambda=\left\{p_{1}, \ldots, p_{N_{0}}\right\}$.

We may give a simple picture of the emergence of the vorticity defects. The first vorticity defects appear close to $H_{c_{1}}$. If $N_{0}=1$ then there is first a unique vorticity defect and it is close to $\Lambda$. If $N_{0} \geqslant 2$ the situation is less clear: we first have $d_{1}^{\star} \in\left\{1, \ldots, N_{0}\right\}$ vorticity defects and each of them is located near one element of $\Lambda$. By increasing the intensity of the applied field $h_{\text {ex }}$ by a bounded quantity we increment the number of vorticity defects until filling $\Lambda$.

Once each elements of $\Lambda$ is close to a vorticity defect, then by increasing $h_{\text {ex }}$ of a $\mathcal{O}(\ln |\ln \varepsilon|)$, additional defects appear one by one.

We may now state the main theorems of the present work. For simplicity of the presentation the theorems are not stated in their most general form (see Theorem 3.3).

(1) In Lemma 4 in [15], $M$ is just a positive number, but $\xi \in C^{0}(\bar{\Omega})$, and then, up to considering $\eta>0$ sufficiently small, we may assume $M \geqslant 1$. 
These main results are obtained assuming that $\lambda, \delta$ and $h_{\text {ex }}$ satisfy

$$
\begin{gathered}
\quad \lambda^{1 / 4}|\ln \varepsilon| \longrightarrow 0 \text { and }|\ln (\lambda \delta)|=\mathcal{O}(\ln |\ln \varepsilon|), \\
\text { There is } K \geqslant 1 \text { s.t. } h_{\mathrm{ex}} \leqslant \frac{b^{2}|\ln \varepsilon|}{2\left\|\xi_{0}\right\|_{L^{\infty}(\Omega)}}+K \ln |\ln \varepsilon|
\end{gathered}
$$

and when $h_{\mathrm{ex}} \rightarrow \infty$ we need

$$
\frac{\ln \left(\delta \sqrt{h_{\mathrm{ex}}}\right)}{\ln \left(\ln h_{\mathrm{ex}}\right)} \rightarrow-\infty
$$

Namely, in order to meet Hypothesis (1.2), (1.3) and (1.4), we may think $\lambda \simeq|\ln \varepsilon|^{-s}, \delta \simeq|\ln \varepsilon|^{-t}$ with $s>4$ and $t>1 / 2$.

We need also assume that

$$
\left\{\begin{array}{l}
\text { the minimal points of } \xi_{0}, \Lambda=\left\{p_{1}, \ldots, p_{N_{0}}\right\}, \\
\text { are non degenerate critical points }
\end{array}\right.
$$

in the sense that for $p \in \Lambda$, letting $\operatorname{Hess}_{\xi_{0}}(p)$ be the Hessian matrix of $\xi_{0}$ at $p$, the quadratic form $Q_{p}(z)=z \cdot \operatorname{Hess}_{\xi_{0}}(p) z$ is a definite positive quadratic form. Note that if (1.5) holds then we may take $M=2$ in Lemma 1.1.

The strategy of this work is based on a perturbative argument. This argument applies for families of quasi-minimizers of the energy with some regularity assumptions (see Theorem 3.3). In particular, we cannot have a sharp profile near a zero of a quasi-minimizer since such profile does not make any sense for quasi-minimizer. Therefore we cannot speak about an ad-hoc notion of vortices s.t. "isolated zeros". However with a natural $L^{\infty}$ bound on the gradient of quasi-minimizers, the notion of vorticity defects is sufficiently robust to give them a nice description.

For simplicity of the presentation we first state the main results for a family $\left\{\left(u_{\varepsilon}, A_{\varepsilon}\right) \mid 0<\varepsilon<1\right\} \subset \mathscr{H}$ s.t.

$$
\left(u_{\varepsilon}, A_{\varepsilon}\right) \text { minimizes } \mathcal{E}_{\varepsilon, h_{\mathrm{ex}}} \text { in } \mathscr{H} \text {. }
$$

Theorem 1.2. - Assume that (1.5) holds and $\lambda, \delta, h_{\mathrm{ex}}, K$ satisfy (1.2), (1.3) and (1.4). There exists $\mathcal{D}_{K, b}>1$ s.t. for $\left\{\left(u_{\varepsilon}, A_{\varepsilon}\right) \mid 0<\varepsilon<1\right\} \subset \mathscr{H}$ satisfying (1.6), for sufficiently small $\varepsilon$, there exists $d_{\varepsilon} \in \mathbb{N}$ s.t. if $d_{\varepsilon}=0$ then $\left|u_{\varepsilon}\right|>b / 2$ in $\Omega$, and if $d_{\varepsilon} \in \mathbb{N}^{*}$ then there exists a set of $d_{\varepsilon}$ points, $\mathcal{Z}_{\varepsilon}=\left\{z_{1}^{\varepsilon}, \ldots, z_{d_{\varepsilon}}^{\varepsilon}\right\} \subset \Omega$, s.t. for $\mu>0$ sufficiently small and independent of $\varepsilon$ we have:

(1) $d_{\varepsilon} \leqslant \mathcal{D}_{K, b}$

(2) $\left\{\left|u_{\varepsilon}\right| \leqslant b / 2\right\} \subset \bigcup B\left(z_{i}^{\varepsilon}, \varepsilon^{\mu}\right) \subset \Omega$,

(3) $\left|z_{i}^{\varepsilon}-z_{j}^{\varepsilon}\right| \geqslant h_{\mathrm{ex}}^{-1} \ln h_{\mathrm{ex}}$ for $i \neq j$, 
(4) $\operatorname{dist}\left(z_{i}^{\varepsilon}, \Lambda\right) \leqslant h_{\mathrm{ex}}^{-1 / 2} \ln h_{\mathrm{ex}}$ for all $i$,

(5) $\operatorname{deg}_{\partial B\left(z_{i}^{\varepsilon}, \varepsilon^{\mu}\right)}\left(u_{\varepsilon}\right)=1$ for all $i$.

Moreover:

(1) There is $\eta_{\omega, b}>0$ depending only on $\omega$ and $b$ s.t., for all $i$, $B\left(z_{i}^{\varepsilon}, \eta_{\omega, b} \lambda \delta\right) \subset \omega_{\varepsilon}$.

(2) If for a sequence $\varepsilon=\varepsilon_{n} \downarrow 0$ we have $h_{\mathrm{ex}}=\mathcal{O}(1)$ then $d_{\varepsilon}=0$ for small $\varepsilon$.

From Theorem 1.2 we know that, for small $\varepsilon$, if $\left\{\left|u_{\varepsilon}\right|<b / 2\right\} \neq \emptyset$, then the vorticity defects are contained in small disks which are well separated, trapped by the impurities and located near $\Lambda$. The second theorem gives sharper informations related with the location of these disks. We divide the second theorem in three parts:

- Macroscopic location: We know that the disks are near $\Lambda$, for some $p \in \Lambda$, how many disks are near $p$ ?

- Mesoscopic location: For $p \in \Lambda$, how are the disks near $p$ organized? What is their inter-distance?

- Microscopic location: We know that the disks are trapped by the inclusion $\omega_{\varepsilon}$, what is their location inside $\omega_{\varepsilon}$.

These questions are related with the crucial notion of renormalized energy (see Section 6).

THEOREM 1.3.

Direct part. - Assume that (1.5) holds and $\lambda, \delta, h_{\mathrm{ex}}, K$ satisfy (1.2), (1.3) and (1.4). Assume also $h_{\mathrm{ex}} \rightarrow \infty$.

Let $\left\{\left(u_{\varepsilon}, A_{\varepsilon}\right) \mid 0<\varepsilon<1\right\} \subset \mathscr{H}$ satisfying (1.6) and let $\varepsilon=\varepsilon_{n} \downarrow 0$ be a sequence. Since $d=d_{\varepsilon} \leqslant \mathcal{D}_{K, b}$, up to passing to a subsequence, we may assume that $d$ is independent of $\varepsilon$. Assume $d>0$.

Macroscopic location. - Recall that $\Lambda=\left\{p_{1}, \ldots, p_{N_{0}}\right\}$ and for $k \in$ $\left\{1, \ldots, N_{0}\right\}$ we let $D_{k}:=\operatorname{deg}_{\partial B\left(p_{k}, 2 \ln \left(h_{\mathrm{ex}}\right) / \sqrt{h_{\mathrm{ex}}}\right)}\left(u_{\varepsilon}\right)$. Write $\mathbf{D}=\left(D_{1}, \ldots\right.$, $\left.D_{N_{0}}\right)$. Up to a subsequence we may assume that $\mathbf{D}$ is independent of $\varepsilon$. We then have:

- The distribution of the disks $B\left(z_{i}^{\varepsilon}, \varepsilon^{\mu}\right)$ around the elements of $\Lambda$ is the most homogenous possible:

$$
\mathbf{D} \in \Lambda_{d}:=\left\{\mathbf{D}^{\prime} \in\left\{\left\lceil\frac{d}{N_{0}}\right\rceil ;\left\lfloor\frac{d}{N_{0}}\right\rfloor\right\}^{N_{0}} \mid \sum_{k=1}^{N_{0}} D_{k}^{\prime}=d\right\} .
$$

Here, for $x \in \mathbb{R}$, we wrote $\lceil x\rceil$ for the ceiling of $x$ and $\lfloor x\rfloor$ for the floor of $x$. 
- There exists a renormalized energy $\mathcal{W}_{d}: \Lambda_{d} \rightarrow \mathbb{R}$ (see (9.16)) s.t. $\mathbf{D}$ minimizes $\mathcal{W}_{d}$.

Mesoscopic location. - The mesoscopic location is the same than in the homogenous case. Namely, for $p \in \Lambda$ s.t. $\operatorname{deg}_{\partial B\left(p, 2 \ln \left(h_{\mathrm{ex}}\right) / \sqrt{h_{\mathrm{ex}}}\right)}\left(u_{\varepsilon}\right)=D>0$, there exists a renormalized energy (see Section 6.2)

$$
W_{p, D}^{\text {meso }}:\left\{\left(a_{1}, \ldots, a_{D}\right) \in\left(\mathbb{R}^{2}\right)^{D} \mid a_{i} \neq a_{j} \text { for } i \neq j\right\} \longrightarrow \mathbb{R}
$$

s.t., denoting $\ell:=\sqrt{\frac{D}{h_{\mathrm{ex}}}}$ and for $z_{i}^{\varepsilon} \in B\left(p, 2 \ln \left(h_{\mathrm{ex}}\right) / \sqrt{h_{\mathrm{ex}}}\right)$ letting $\breve{z}_{i}^{\varepsilon}:=$ $\frac{z_{i}^{\varepsilon}-p}{\ell}$, we have $\breve{\mathbf{z}}^{\varepsilon}=\left(\breve{z}_{1}^{\varepsilon}, \ldots, \breve{z}_{D}^{\varepsilon}\right)$ (assuming $z_{i}^{\varepsilon} \in B\left(p, 2 \ln \left(h_{\mathrm{ex}}\right) / \sqrt{h_{\mathrm{ex}}}\right) \Leftrightarrow i \in$ $\{1, \ldots, D\})$ which converges to a minimizer of $W_{p, D}^{\text {meso }}$. In particular $\ell$ is the typical interdistance between two close $z_{i}^{\varepsilon}, z_{j}^{\varepsilon}$.

Microscopic location. - We know that, for $i \in\{1, \ldots, d\}, B\left(z_{i}^{\varepsilon}, \eta_{\omega, b} \lambda \delta\right) \subset$ $\omega_{\varepsilon}$. Moreover for $i \neq j$ we have $\left|z_{i}^{\varepsilon}-z_{j}^{\varepsilon}\right| \geqslant \ln \left(h_{\mathrm{ex}}\right) h_{\mathrm{ex}}^{-1} \gg \lambda \delta$. Then each connected component of $\omega_{\varepsilon}$ contains at most one disk $B\left(z_{i}^{\varepsilon}, \varepsilon^{\mu}\right)$.

There exists a renormalized energy $W^{\text {micro }}: \omega \rightarrow \mathbb{R}$ (see Section 6.3) s.t. for $i \in\{1, \ldots, d\}$, letting $y_{i}^{\varepsilon} \in \delta \cdot \mathbb{Z}^{2}$ be s.t. $B\left(z_{i}^{\varepsilon}, \eta_{\omega, b} \lambda \delta\right) \subset y_{i}^{\varepsilon}+\lambda \delta \omega$ and $\widehat{z}_{i}^{\varepsilon}:=\frac{z_{i}^{\varepsilon}-y_{i}^{\varepsilon}}{\lambda \delta} \in \omega$ we have

- $W^{\text {micro }}\left(\widehat{z}_{i}^{\varepsilon}\right) \rightarrow \min _{\omega} W^{\text {micro }}$,

- Up to passing to a subsequence, there is $a_{i} \in \omega$ s.t. $\widehat{z}_{i}^{\varepsilon} \rightarrow a_{i}$ and $a_{i}$ minimizes $W^{\text {micro }}{ }^{(2)}$

Optimality of the renormalized energies. - Consider a sequence $\varepsilon=$ $\varepsilon_{n} \downarrow 0$ previously fixed (in order to have $\mathbf{D}$ independent of $\varepsilon$ ) and assume $d \neq 0$. We let

- $\mathbf{D}^{\prime} \in \Lambda_{d}$ be a minimizer of $\mathcal{W}_{d}$,

- for $k \in\left\{1, \ldots, N_{0}\right\}$ s.t. $D_{k}^{\prime} \geqslant 1, \mathbf{a}_{k}^{\prime}$ be a minimizer of $W_{p_{k}, D_{k}^{\prime}}^{\text {meso }}$,

- $a_{0}$ be a minimizer of $W^{\text {micro }}$.

Then, for $\varepsilon=\varepsilon_{n}$, there exist $\left(u_{\varepsilon}^{\prime}, A_{\varepsilon}^{\prime}\right) \in \mathscr{H}$ and distinct points of $\Omega$, $\left\{z_{1}^{\prime}, \ldots, z_{d}^{\prime}\right\}=\left\{z_{1}^{\varepsilon \prime}, \ldots, z_{d}^{\varepsilon^{\prime}}\right\} \subset \omega_{\varepsilon}$, s.t.

- $\mathcal{E}_{\varepsilon, h_{\mathrm{ex}}}\left(u_{\varepsilon}^{\prime}, A_{\varepsilon}^{\prime}\right) \leqslant \inf \mathscr{H}_{\mathcal{E}} \mathcal{E}_{\varepsilon, h_{\mathrm{ex}}}+o(1)$,

- $\left\{\left|u_{\varepsilon}^{\prime}\right| \leqslant b / 2\right\} \subset \bigcup B\left(z_{i}^{\prime}, \sqrt{\varepsilon}\right) \subset \bigcup_{p \in \Lambda} B\left(p, \ln \left(h_{\mathrm{ex}}\right) / \sqrt{h_{\mathrm{ex}}}\right)$,

- for $k \in\left\{1, \ldots, N_{0}\right\}, D_{k}^{\prime}=\operatorname{deg}_{\partial B\left(p_{k}, 2 \ln \left(h_{\mathrm{ex}}\right) / \sqrt{h_{\mathrm{ex}}}\right)}\left(u_{\varepsilon}^{\prime}\right)$,

- $\operatorname{deg}_{\partial B\left(z_{i}^{\prime}, \sqrt{\varepsilon}\right)}\left(u_{\varepsilon}^{\prime}\right)=1$ for all $i$,

(2) For example if $\omega$ is a disk then $a_{i}$ is the center of the disk [7]. 
- writing for $p_{k} \in \Lambda$ (s.t. $\left.D_{k}^{\prime} \geqslant 1\right)$ and $z_{i}^{\prime} \in B\left(p_{k}, \ln \left(h_{\mathrm{ex}}\right) / \sqrt{h_{\mathrm{ex}}}\right)$, $\breve{z}_{i}^{\prime}:=\left(z_{i}-p_{k}\right) / \sqrt{D_{k} / h_{\mathrm{ex}}}$ and $\breve{\mathbf{z}}_{p_{k}}^{\prime}:=\left\{\breve{z}_{i}^{\prime} \mid z_{i}^{\prime} \rightarrow p_{k}\right\},{ }^{(3)}$ we have $\breve{\mathbf{z}}_{p_{k}}^{\prime} \rightarrow \mathbf{a}_{k}^{\prime}$

- For $i \in\{1, \ldots, d\}$, letting $y_{i}^{\varepsilon} \in \delta \cdot \mathbb{Z}^{2}$ be s.t. $z_{i}^{\prime} \in y_{i}^{\varepsilon}+\lambda \delta \cdot \omega$ and $\widehat{z}_{i}^{\prime}:=\frac{z_{i}^{\prime}-y_{i}^{\varepsilon}}{\lambda \delta} \in \omega$ we have $\widehat{z}_{i}^{\prime} \rightarrow a_{0}$.

The third theorem underlines the link between the number $d$ and $h_{\mathrm{ex}}$.

TheOrem 1.4. - Assume that $\Omega$ satisfies (1.5), $\lambda, \delta, h_{\mathrm{ex}}, K$ satisfy (1.2), (1.3) and (1.4).

There are integers $L \in\left\{1, \ldots, N_{0}\right\}, 0=d_{0}^{\star}<d_{1}^{\star}<\cdots<d_{L}^{\star}=N_{0}$ $\left(d_{k}^{\star} \in \mathbb{N}\right.$ is independent of $\varepsilon$ ) and critical fields (depending on $\varepsilon$ ) $\mathrm{K}_{1}^{(\mathrm{I})}<\cdots<$ $\mathrm{K}_{L}^{(\mathrm{I})}<\mathrm{K}_{1}^{(\mathrm{II})}<\mathrm{K}_{2}^{(\mathrm{II})}<\cdots\left(\right.$ see $(10.9)$ and $(10.10)$ for the expressions of $\mathrm{K}_{k}^{(\mathrm{I})}$ and $\left.\mathrm{K}_{k}^{(\mathrm{II})}\right)$ s.t. for $\left\{\left(u_{\varepsilon}, A_{\varepsilon}\right) \mid 0<\varepsilon<1\right\} \subset \mathscr{H}$ a family satisfying (1.6) and for a sequence $\varepsilon=\varepsilon_{n} \downarrow 0$ :

- If $d_{\varepsilon}=0$ for small $\varepsilon$, then $h_{\mathrm{ex}} \leqslant \mathrm{K}_{1}^{(\mathrm{I})}+o(1)$.

- If $d_{\varepsilon}>0$ for small $\varepsilon$, then $h_{\mathrm{ex}} \geqslant \mathrm{K}_{1}^{(\mathrm{I})}+o(1)$.

- Assume $L \geqslant 2$. For $k \in\{1, \ldots, L-1\}$, if for small $\varepsilon$ we have $d_{k-1}^{\star}<d_{\varepsilon} \leqslant d_{k}^{\star}$, then

$$
\mathrm{K}_{k}^{(\mathrm{I})}+o(1) \leqslant h_{\mathrm{ex}} \leqslant \mathrm{K}_{k+1}^{(\mathrm{I})}+o(1) .
$$

- For $L \geqslant 1$, if for small $\varepsilon$ we have $d_{L-1}^{\star}<d_{\varepsilon} \leqslant d_{L}^{\star}=N_{0}$, then

$$
\mathrm{K}_{L}^{(\mathrm{I})}+o(1) \leqslant h_{\mathrm{ex}} \leqslant \mathrm{K}_{1}^{(\mathrm{II})}+o(1) .
$$

- Let $l \in \mathbb{N}^{*}$. If for small $\varepsilon$ we have $d_{\varepsilon}=N_{0}+l$, then

$$
\mathrm{K}_{l}^{(\mathrm{II})}+o(1) \leqslant h_{\mathrm{ex}} \leqslant \mathrm{K}_{l+1}^{(\mathrm{II})}+o(1) .
$$

Remark 1.5. - A more complete statement for $d_{\varepsilon} \in\left\{1, \ldots, N_{0}\right\}$ may be found in Proposition 10.7.

\section{Acknowledgments}

The author would like to thank Lia Bronsard, Vincent Millot, Petru Mironescu and Etienne Sandier for fruitful discussions. The author would like to thank also the anonymous referee for their helpful comments.

(3) We used a little abuse of notation for the simplicity of the presentation. 


\section{Notation}

\subsection{Sets, vectors and numbers}

- We identify the real plan $\mathbb{R}^{2}$ with $\mathbb{C}$ and we denote by $\mathbb{S}^{1}$ the unit circle in $\mathbb{C}$.

- For $\mathscr{U} \subset \mathbb{R}^{2}, N \in \mathbb{N} \backslash\{0 ; 1\},\left(\mathscr{U}^{N}\right)^{*}:=\left\{\left(z_{1}, \ldots, z_{N}\right) \in \mathscr{U}^{N} \mid z_{i} \neq\right.$ $z_{j}$ for $\left.i \neq j\right\}$.

- For $k \in\{1 ; 2\}, \mathcal{H}^{k}$ is the $k$-dimensional Hausdorff measure.

- If $\left(a_{1}, a_{2}\right),\left(b_{1}, b_{2}\right) \in \mathbb{R}^{2}$, then $\left|\left(a_{1}, a_{2}\right)\right|=\sqrt{a_{1}^{2}+a_{2}^{2}},\left(a_{1}, a_{2}\right)^{\perp}=$ $\left(-a_{2}, a_{1}\right),\left(a_{1}, a_{2}\right) \cdot\left(b_{1}, b_{2}\right)=a_{1} b_{1}+a_{2} b_{2}$ and $\left(a_{1}, a_{2}\right) \wedge\left(b_{1}, b_{2}\right)=$ $a_{1} b_{2}-a_{2} b_{1}$.

- For $\mathscr{U} \subset \mathbb{R}^{2}, \overline{\mathscr{U}}$ is the closure of $\mathscr{U}$ w.r.t. $|\cdot|$

- For $\emptyset \neq \mathscr{U}, \mathscr{V} \subset \mathbb{R}^{2}$ and $x_{0} \in \mathbb{R}^{2}$ we write $\operatorname{dist}(\mathscr{U}, \mathscr{V}):=\inf \{|x-y| \mid$ $x \in \mathscr{U}, y \in \mathscr{V}\}$ and $\operatorname{dist}\left(x_{0}, \mathscr{V}\right):=\operatorname{dist}\left(\left\{x_{0}\right\}, \mathscr{V}\right)$.

- For $\Gamma \subset \mathbb{R}^{2}$ a Jordan curve we let:

- $\operatorname{int}(\Gamma)$, the interior of $\Gamma$, be the bounded open set $\mathscr{U} \subset \mathbb{R}^{2}$ s.t. $\Gamma=\partial \mathscr{U}$ where $\partial \mathscr{U}$ is the boundary of $\mathscr{U}$.

$-\nu$ be the outward normal unit vector of int $(\Gamma)$

$-\tau$ be the direct unit tangent vector of $\Gamma\left(\tau=\nu^{\perp}\right)$

- If $S$ is a finite set then $\operatorname{Card}(S)$ is the cardinal of $S$.

- If $x \in \mathbb{R}$, then we write $\lceil x\rceil:=\min \{m \in \mathbb{Z} \mid m \geqslant x\}$, the ceiling of $x$, and $\lfloor x\rfloor:=\max \{m \in \mathbb{Z} \mid m \leqslant x\}$, the floor of $x$.

\subsection{Functions}

- When $\mathscr{U} \subset \mathbb{R}^{2}$ is a smooth bounded open set we write $H^{1}(\mathscr{U}, \mathbb{C})$ for the Classical Sobolev space of the first order modeled on the Lebesgue space $L^{2}$ and, for $K \subset \mathbb{C}, H^{1}(\mathscr{U}, K):=\left\{u \in H^{1}(\mathscr{U}, \mathbb{C}) \mid\right.$ $u(x) \in K$ for a.e. $x \in \mathscr{U}\}$.

For $k \in \mathbb{N}^{*}$ and $p \in[1, \infty]$ we use the standard notation for the higher order Sobolev spaces $H^{k}(\mathscr{U}, K)$ modeled on $L^{2}$ and $W^{k, p}(\mathscr{U}, K)$ for the Sobolev space of order $k$ modeled on $L^{p}$.

- We use the standard notation for the differential operators: " $\nabla$ " for the gradient, "curl" for the curl, "div" for the divergence, " $\partial_{\tau}=$ $\tau \cdot \nabla$ " for the tangential derivative, " $\partial_{\nu}=\nu \cdot \nabla$ " for the normal derivative...

- We let $\operatorname{tr}_{\partial \mathscr{U}}: H^{1}(\mathscr{U}, \mathbb{C}) \rightarrow H^{1 / 2}(\partial \mathscr{U}, \mathbb{C})$ be the (surjective) trace operator. For $\Gamma$ a connected component of $\partial \mathscr{U}$ and $u \in H^{1}(\mathscr{U}, \mathbb{C})$, we let $\operatorname{tr}_{\Gamma}(u)$ be the restriction of $\operatorname{tr}_{\partial \mathscr{U}}(u)$ to $\Gamma$.

We write $H_{0}^{1}(\mathscr{U}, \mathbb{C}):=\left\{u \in H^{1}(\mathscr{U}, \mathbb{C}) \mid \operatorname{tr}_{\partial \mathscr{U}}(u)=0\right\}$. 
- For $u: \Omega \rightarrow \mathbb{C}$ a function we let

$$
\underline{u}:= \begin{cases}u & \text { if }|u| \leqslant 1 \\ u /|u| & \text { if }|u|>1 .\end{cases}
$$

- For $\Gamma \subset \mathbb{R}^{2}$ a Jordan curve and $g \in H^{1 / 2}\left(\Gamma, \mathbb{S}^{1}\right)$, the degree of $g$ is defined as

$$
\operatorname{deg}_{\Gamma}(g):=\frac{1}{2 \pi} \int_{\Gamma} g \wedge \partial_{\tau} g \in \mathbb{Z} .
$$

For a smooth and bounded open set $\mathscr{U} \subset \mathbb{R}^{2}, \Gamma$ a connected component of $\partial \mathscr{U}$ and $u \in H^{1}(\mathscr{U}, \mathbb{C})$, if there exists $\eta>0$ s.t. $g:=\operatorname{tr}_{\Gamma}(u)$ satisfies $|g| \geqslant \eta$, then $g /|g| \in H^{1 / 2}\left(\Gamma, \mathbb{S}^{1}\right)$ and we write $\operatorname{deg}_{\Gamma}(u):=$ $\operatorname{deg}_{\Gamma}(g /|g|)$.

When $\mathscr{U}, \mathscr{V} \subset \mathbb{R}^{2}$ are smooth bounded simply connected open sets s.t. $\overline{\mathscr{V}} \subset \mathscr{U}$ and $u \in H^{1}\left(\mathscr{U} \backslash \overline{\mathscr{V}}, \mathbb{S}^{1}\right)$, then we write (without ambiguity) $\operatorname{deg}(u)$ instead of $\operatorname{deg}_{\Gamma}(u)$ for any Jordan curve $\Gamma \subset$ $\overline{\mathscr{U}} \backslash \mathscr{V}$ s.t. $\mathscr{V} \subset \operatorname{int}(\Gamma)$.

\subsection{Construction of the pinning term}

Let

- $\delta=\delta(\varepsilon) \in(0,1), \lambda=\lambda(\varepsilon) \in(0,1)$;

- $\omega \subset \mathbb{R}^{2}$ be a smooth bounded and simply connected open set s.t. $(0,0) \in \omega$ and $\bar{\omega} \subset Y:=(-1 / 2,1 / 2)^{2}$.

For $m \in \mathbb{Z}^{2}$ we denote $Y_{m}^{\delta}:=\delta m+\delta \cdot Y$ and

$$
\omega_{\varepsilon}=\bigcup_{\substack{m \in \mathbb{Z}^{2} \\ Y_{m}^{\delta} \subset \Omega}}[\delta m+\lambda \delta \cdot \omega] .
$$

For $b \in(0,1)$ we may now define the pinning term

$$
\begin{aligned}
a_{\varepsilon}: \mathbb{R}^{2} & \longrightarrow\{b, 1\}, \\
x & \longmapsto \begin{cases}b & \text { if } x \in \omega_{\varepsilon}, \\
1 & \text { otherwise. }\end{cases}
\end{aligned}
$$

\subsection{Asymptotic}

- In this article $\varepsilon \in(0,1)$ is a small number. We are essentially interested in the asymptotic $\varepsilon \rightarrow 0$. In order to keep simple notation we will often omit to mention the parameter $\varepsilon$. 
- When we consider a sequence $\left(\varepsilon_{n}\right)_{n} \subset(0,1)$ s.t. $\varepsilon_{n} \downarrow 0$ we often omit the mention of the index $n$ writing $\varepsilon=\varepsilon_{n}$.

- The notation $o(1)$ means a quantity depending on $\varepsilon$ which tends to 0 when $\varepsilon \rightarrow 0$.

- For $f:(0,1) \rightarrow(0,+\infty)$, the notation $o[f(\varepsilon)]$ means a quantity $g(\varepsilon)$ s.t. $g(\varepsilon) / f(\varepsilon)=o(1)$ and $\mathcal{O}[f(\varepsilon)]$ means a quantity $g(\varepsilon)$ s.t. $g(\varepsilon) / f(\varepsilon)$ is bounded for small $\varepsilon$.

\section{Classical facts and the strongest theorem}

\section{Gauge invariance and Coulomb Gauge}

It is standard to quote the gauge invariance of the energy $\mathcal{E}_{\varepsilon, h_{\mathrm{ex}}}$. Namely, two configurations $(u, A),\left(u^{\prime}, A^{\prime}\right) \in \mathscr{H}$ are gauge equivalent, denoted by $(u, A) \stackrel{\text { gauge }}{\sim}\left(u^{\prime}, A^{\prime}\right)$, if there exists a gauge transformation from $(u, A)$ to $\left(u^{\prime}, A^{\prime}\right)$ :

$$
(u, A) \stackrel{\text { gauge }}{\sim}\left(u^{\prime}, A^{\prime}\right) \Longleftrightarrow \exists \varphi \in H^{2}(\Omega, \mathbb{R}) \text { s.t. } u^{\prime}=u \mathrm{e}^{\imath \varphi} \text { and } A^{\prime}=A+\nabla \varphi .
$$

Two gauge equivalent configurations describe the same physical state. Then, physical quantities are those which are gauge invariant. For example, if $(u, A) \in \mathscr{H}$, then $|u|,|\nabla u-\imath A u|, \operatorname{curl}(A)$ and then $\mathcal{E}_{\varepsilon, h_{\text {ex }}}(u, A),\{|u| \leqslant b / 2\}$ also are gauge invariants. Note that the main results of the present work are gauge invariant.

In the context the Ginzburg-Landau energy, a classical choice of gauge is the Coulomb gauge. We say that $(u, A)$ is in the Coulomb gauge if

$$
\begin{cases}\operatorname{div}(A)=0 & \text { in } \Omega \\ A \cdot \nu=0 & \text { on } \partial \Omega .\end{cases}
$$

One may prove (see [16, Proposition 3.2]) that, for $(u, A) \in \mathscr{H}$, there exists $\varphi \in H^{2}(\Omega, \mathbb{R})$ s.t. $A^{\prime}:=A+\nabla \varphi$ satisfies (3.1). Then, letting $u^{\prime}=u \mathrm{e}^{\imath \varphi}$, we have $\left(u^{\prime}, A^{\prime}\right)$ which is in the Coulomb gauge and $(u, A) \stackrel{\text { gauge }}{\sim}\left(u^{\prime}, A^{\prime}\right)$.

One of the main motivations in using the Coulomb gauge comes from the fact that $\|\operatorname{curl}(A)\|_{L^{2}}$ controls $\|A\|_{H^{1}}$. Namely there exists $C \geqslant 1$ (which depends only on $\Omega$ ) s.t. if $A$ satisfies (3.1) then (see [16, Proposition 3.3])

$$
\|A\|_{H^{1}\left(\Omega, \mathbb{R}^{2}\right)} \leqslant C\|\operatorname{curl}(A)\|_{L^{2}(\Omega)}
$$

and

$$
\|A\|_{H^{2}\left(\Omega, \mathbb{R}^{2}\right)} \leqslant C\|\operatorname{curl}(A)\|_{H^{1}(\Omega)} .
$$


Moreover we have an easy representation of $A \in H^{1}\left(\Omega, \mathbb{R}^{2}\right)$ satisfying (3.1) $A \in H^{1}\left(\Omega, \mathbb{R}^{2}\right)$ is a solution of $(3.1)$

$$
\Longleftrightarrow \exists \xi \in H_{0}^{1} \cap H^{2}(\Omega, \mathbb{R}) \text { s.t. } A=\nabla^{\perp} \xi .
$$

\section{Basic description of a minimizer}

We first note that, by direct minimization, for all $a_{\varepsilon} \in L^{\infty}(\Omega,[b, 1])$, $\varepsilon, h_{\mathrm{ex}}>0$, the minimization problem of $\mathcal{E}_{\varepsilon, h_{\mathrm{ex}}}$ in $\mathscr{H}$ admits (at least) a solution $\left(u_{\varepsilon}, A_{\varepsilon}\right) \in \mathscr{H}$.

Writing $h_{\varepsilon}:=\operatorname{curl}\left(A_{\varepsilon}\right)$, it is standard to check that a such minimizer solves:

$$
\begin{cases}-\left(\nabla-\imath A_{\varepsilon}\right)^{2} u_{\varepsilon}=\frac{u_{\varepsilon}}{\varepsilon^{2}}\left(a_{\varepsilon}^{2}-\left|u_{\varepsilon}\right|^{2}\right) & \text { in } \Omega \\ (\nabla-\imath A) u_{\varepsilon} \cdot \nu=0 & \text { on } \partial \Omega \\ -\nabla^{\perp} h_{\varepsilon}=u_{\varepsilon} \wedge\left(\nabla-\imath A_{\varepsilon}\right) u_{\varepsilon} & \text { in } \Omega \\ h_{\varepsilon}=h_{\mathrm{ex}} & \text { on } \partial \Omega .\end{cases}
$$

Using a maximum principle, we may get the following proposition:

Proposition 3.1. - Let $\varepsilon, h_{\mathrm{ex}}>0$ and $a \in L^{\infty}(\Omega,[b, 1])$. If $\left(u_{\varepsilon}, A_{\varepsilon}\right)$ is a minimizer of $\mathcal{E}(u, A)=\frac{1}{2} \int_{\Omega}|\nabla u-\imath A u|^{2}+\frac{1}{2 \varepsilon^{2}}\left(a^{2}-|u|^{2}\right)^{2}+\left|\operatorname{curl}(A)-h_{\mathrm{ex}}\right|^{2}$ in $\mathscr{H}$ then $\left|u_{\varepsilon}\right| \leqslant 1$ in $\Omega$.

On the other hand, if $\left(u_{\varepsilon}, A_{\varepsilon}\right)$ is a minimizer of $\mathcal{E}_{\varepsilon, h_{\mathrm{ex}}}$ in the Coulomb gauge, then it solves

$$
\begin{cases}-\Delta u_{\varepsilon}=\frac{u_{\varepsilon}}{\varepsilon^{2}}\left(a_{\varepsilon}^{2}-\left|u_{\varepsilon}\right|^{2}\right)-2 \imath\left(A_{\varepsilon} u_{\varepsilon} \cdot \nabla u_{\varepsilon}\right)-\left|A_{\varepsilon}\right|^{2} u_{\varepsilon} & \text { in } \Omega \\ \partial_{\nu} u_{\varepsilon}=0 & \text { on } \Omega .\end{cases}
$$

A fundamental bound in the study concerns $\left\|\nabla u_{\varepsilon}\right\|_{L^{\infty}(\Omega)}$. We have the following lemma which is a Gagliardo-Nirenberg type inequality with homogenous Neumann boundary condition.

LEMMA 3.2. — ${ }^{(4)}$ Let $\Omega \subset \mathbb{R}^{2}$ be a smooth bounded simply connected open set. There exists $C_{\Omega} \geqslant 1$ s.t. if $u \in H^{2}(\Omega)$ is s.t. $\partial_{\nu} u=0$ on $\partial \Omega$ then

$$
\|\nabla u\|_{L^{\infty}(\Omega)}^{2} \leqslant C_{\Omega}\left(\|\Delta u\|_{L^{\infty}(\Omega)}+\|u\|_{L^{\infty}(\Omega)}\right)\|u\|_{L^{\infty}(\Omega)} .
$$

(4) The proof of Lemma 3.2 is done by first using $\Phi: \mathbb{D} \rightarrow \Omega$, a conformal representation of $\Omega$ on the unit disk $\mathbb{D}$. Then we extend $\widetilde{u}:=u \circ \Phi$ in the disk $B(0,2)$ by letting $u^{\prime}(x)=$ $\widetilde{u}(x /|x|)$ for $x \in B(0,2) \backslash \mathbb{D}$. By using the boundary condition we have $u^{\prime} \in H^{2}(B(0,2), \mathbb{C})$. And finally one may conclude by using an interior version of Lemma 3.2 (Lemma A.1 in $[3])$. 
Consequently, with Lemma 3.2 (up to changing the value of $C_{\Omega}$ ), for $\varepsilon, h_{\mathrm{ex}}>0$ and $a_{\varepsilon} \in L^{\infty}\left(\Omega,\left[b^{2}, 1\right]\right)$, if $\left(u_{\varepsilon}, A_{\varepsilon}\right) \in \mathscr{H}$ minimizes $\mathcal{E}_{\varepsilon, h_{\mathrm{ex}}}$ is in the Coulomb gauge and is s.t. $\left\|A_{\varepsilon}\right\|_{L^{\infty}(\Omega)} \leqslant 1 / \varepsilon$ (which is the case in the present work) then

$$
\left\|\nabla u_{\varepsilon}\right\|_{L^{\infty}(\Omega)} \leqslant \frac{C_{\Omega}}{\varepsilon} .
$$

In the homogenous case as well as in the case without magnetic field, Estimate (3.7) is crucial to describe vorticity defects. It is the same in the present work. More precisely, the main result (Theorem 3.3) states that the three above theorems are true replacing $\left(u_{\varepsilon}, A_{\varepsilon}\right)$ that minimizes $\mathcal{E}_{\varepsilon, h_{\text {ex }}}$ in $\mathscr{H}$ by any configuration $\left(\widetilde{u}_{\varepsilon}, \widetilde{A}_{\varepsilon}\right)$ s.t. $E_{\varepsilon}\left(\widetilde{u}_{\varepsilon}, \widetilde{A}_{\varepsilon}\right)=\inf \mathscr{H} \mathcal{E}_{\varepsilon, h_{\text {ex }}}+o(1)$ with two extra hypotheses on $\left|\widetilde{u}_{\varepsilon}\right|:\left\|\nabla\left|\widetilde{u}_{\varepsilon}\right|\right\|_{L^{\infty}(\Omega)}=\mathcal{O}\left(\varepsilon^{-1}\right)$ and $\left|\widetilde{u}_{\varepsilon}\right| \in W^{2,1}(\Omega)$ (see (3.11))

\section{Lassoued-Mironescu decoupling}

In order to study pinned Ginzburg-Landau type energies, a nice trick was initiated by Lassoued and Mironescu in [12]. Before explaining this trick we have to do a direct calculation for $(u, A) \in \mathscr{H}$ :

$$
\mathcal{E}_{\varepsilon, h_{\mathrm{ex}}}(u, A)=E_{\varepsilon}(u)+\frac{1}{2} \int_{\Omega}-2(u \wedge \nabla u) \cdot A+|u|^{2}|A|^{2}+\left|\operatorname{curl}(A)-h_{\mathrm{ex}}\right|^{2}
$$

with

$$
E_{\varepsilon}(u)=\frac{1}{2} \int_{\Omega}|\nabla u|^{2}+\frac{1}{2 \varepsilon^{2}}\left(a_{\varepsilon}^{2}-|u|^{2}\right)^{2} .
$$

The Lassoued-Mironescu decoupling is obtained by first minimizing $E_{\varepsilon}$ in $H^{1}(\Omega, \mathbb{C})$. It is clear that $E_{\varepsilon}$ admits minimizers and if $U$ minimizes $E_{\varepsilon}$ then it satisfies

$$
\begin{cases}-\Delta U=\frac{U}{\varepsilon^{2}}\left(a_{\varepsilon}^{2}-|U|^{2}\right) & \text { in } \Omega \\ \partial_{\nu} U=0 & \text { on } \partial \Omega .\end{cases}
$$

By an energetic argument it is easy to prove that, if $U$ minimizes $E_{\varepsilon}$ in $H^{1}(\Omega, \mathbb{C})$, then $b \leqslant|U| \leqslant 1$. Moreover from (3.9), $U \wedge \nabla U=0$, i.e. $U=|U| \mathrm{e}^{\imath \theta}$ with $\theta \in \mathbb{R}$.

Then one may consider a scalar minimizer $U_{\varepsilon}: \Omega \rightarrow[b, 1]$. This scalar minimizer may be seen as a regularization of $a_{\varepsilon}$ (see Proposition 5.2).

Using this scalar minimizer one may get the well known LassouedMironescu decoupling: for $v \in H^{1}(\Omega, \mathbb{C})$ we have

$$
E_{\varepsilon}\left(U_{\varepsilon} v\right)=E_{\varepsilon}\left(U_{\varepsilon}\right)+F_{\varepsilon}(v)
$$


with

$$
F_{\varepsilon}(v):=\frac{1}{2} \int_{\Omega} U_{\varepsilon}^{2}|\nabla v|^{2}+\frac{U_{\varepsilon}^{4}}{2 \varepsilon^{2}}\left(1-|v|^{2}\right)^{2} .
$$

Using this decoupling, one may prove that, for $\varepsilon>0$, there exists a unique positive minimizer $U_{\varepsilon}: \Omega \rightarrow[b, 1]$ of $E_{\varepsilon}$ in $H^{1}(\Omega, \mathbb{R})$.

On the other hand, from (3.8) and (3.10), for $(u, A) \in \mathscr{H}$ and $v=u / U_{\varepsilon}$ we have:

$$
\begin{aligned}
\mathcal{F}_{\varepsilon, h_{\mathrm{ex}}}(v, A): & =\mathcal{E}_{\varepsilon, h_{\mathrm{ex}}}\left(U_{\varepsilon} v, A\right)-E_{\varepsilon}\left(U_{\varepsilon}\right) \\
& =\frac{1}{2} \int_{\Omega} U_{\varepsilon}^{2}|\nabla v-\imath A v|^{2}+\frac{U_{\varepsilon}^{4}}{2 \varepsilon^{2}}\left(1-|v|^{2}\right)^{2}+\left|\operatorname{curl}(A)-h_{\mathrm{ex}}\right|^{2} .
\end{aligned}
$$

It is easy to check that $\mathcal{F}_{\varepsilon, h_{\mathrm{ex}}}(v, A)$ is gauge invariant. This functional is of major interest in the study since $(v, A)$ minimizes $\mathcal{F}_{\varepsilon, h_{\mathrm{ex}}}$ in $\mathscr{H}$ if and only if $\left(U_{\varepsilon} v, A\right)$ minimizes $\mathcal{E}_{\varepsilon, h_{\mathrm{ex}}}$ in $\mathscr{H}$.

An easy comparaison argument implies that if $\left(v_{\varepsilon}, A_{\varepsilon}\right)$ minimizes $\mathcal{F}_{\varepsilon, h_{\mathrm{ex}}}$ then $\left\|v_{\varepsilon}\right\|_{L^{\infty}(\Omega)} \leqslant 1$.

From now on we focus on the study of the minimizer of $\mathcal{F}_{\varepsilon, h_{\mathrm{ex}}}$. Namely we have the following theorem.

TheOrem 3.3. - Assume that (1.5) holds and $\lambda, \delta, h_{\mathrm{ex}}, K$ satisfy (1.2), (1.3) and (1.4).

Let $\left\{\left(v_{\varepsilon}, A_{\varepsilon}\right) \mid 0<\varepsilon<1\right\} \subset \mathscr{H}$ be s.t. $\mathcal{F}\left(v_{\varepsilon}, A_{\varepsilon}\right) \leqslant \inf \mathscr{H} \mathcal{F}+o(1)$. Assume also that

$$
\left\{\begin{array}{l}
\left|v_{\varepsilon}\right| \in W^{2,1}(\Omega, \mathbb{C}) \\
\left\|\nabla\left|v_{\varepsilon}\right|\right\|_{L^{\infty}(\Omega)}=\mathcal{O}\left(\varepsilon^{-1}\right) .
\end{array}\right.
$$

Then Theorems 1.2, 1.3 and 1.4 hold for $u_{\varepsilon}=U_{\varepsilon} v_{\varepsilon}$.

Remark 3.4. - Theorem 3.3 may be rephrased in term of $U_{\varepsilon}$. Let $\left(h_{\mathrm{ex}}\right)_{0<\varepsilon<1} \subset(0, \infty),\left\{\left(u_{\varepsilon}, A_{\varepsilon}\right) \mid 0<\varepsilon<1\right\} \subset \mathscr{H}$ and let $v_{\varepsilon}:=u_{\varepsilon} / U_{\varepsilon} \in$ $H^{1}(\Omega, \mathbb{C})$. On the one hand, from the decoupling $(3.10)$, we have $\left\{\left(u_{\varepsilon}, A_{\varepsilon}\right) \mid\right.$ $0<\varepsilon<1\} \subset \mathscr{H}$ is s.t. $\mathcal{E}_{\varepsilon, h_{\mathrm{ex}}}\left(u_{\varepsilon}, A_{\varepsilon}\right) \leqslant \inf _{\mathscr{H}} \mathcal{E}_{\varepsilon, h_{\mathrm{ex}}}+o(1)$ if and only $\left\{\left(v_{\varepsilon}, A_{\varepsilon}\right) \mid 0<\varepsilon<1\right\}$ is s.t. $\mathcal{F}_{\varepsilon, h_{\mathrm{ex}}}\left(v_{\varepsilon}, A_{\varepsilon}\right) \leqslant \inf _{\mathscr{H}} \mathcal{F}_{\varepsilon, h_{\mathrm{ex}}}+o(1)$. On the other hand, if $\left(v_{\varepsilon}\right)_{\varepsilon}$ is bounded in $L^{\infty}(\Omega)$, then $v_{\varepsilon}$ satisfies (3.11) if and only if we have $\left|u_{\varepsilon}\right| \in W^{2,1}(\Omega, \mathbb{C})$ and $\left\|\nabla\left|u_{\varepsilon}\right|\right\|_{L^{\infty}(\Omega)}=\mathcal{O}\left(\varepsilon^{-1}\right)$.

\section{Plan of the article and proof of Theorem 3.3}

The proof of Theorem 3.3 is done in several steps. It is based on a perturbative argument by replacing the energy $\mathcal{F}_{\varepsilon, h_{\text {ex }}}$ with an energy $\widetilde{\mathcal{F}}_{\varepsilon, h_{\mathrm{ex}}}$. This 
step is called the energetic cleaning (Section 5.1). The functional $\widetilde{\mathcal{F}}_{\varepsilon, h_{\mathrm{ex}}}$ is a perturbation of $\mathcal{F}_{\varepsilon, h_{\text {ex }}}$ : for $\left(v_{\varepsilon}, A_{\varepsilon}\right) \in \mathscr{H}$ which is in the Coulomb gauge and s.t. $\mathcal{F}_{\varepsilon, h_{\mathrm{ex}}}\left(v_{\varepsilon}, A_{\varepsilon}\right)=\mathcal{O}\left(h_{\mathrm{ex}}^{2}\right)$ we have $\widetilde{\mathcal{F}}_{\varepsilon, h_{\mathrm{ex}}}\left(v_{\varepsilon}, A_{\varepsilon}\right)-\mathcal{F}_{\varepsilon, h_{\mathrm{ex}}}\left(v_{\varepsilon}, A_{\varepsilon}\right)=o(1)$ (see Proposition 5.3). In particular we have $\mathcal{F}_{\varepsilon, h_{\text {ex }}}\left(v_{\varepsilon}, A_{\varepsilon}\right) \leqslant \inf \mathcal{H}_{\mathcal{H}} \mathcal{F}_{\varepsilon, h_{\text {ex }}}+$ $o(1)$ if and only if $\widetilde{\mathcal{F}}_{\varepsilon, h_{\text {ex }}}\left(v_{\varepsilon}, A_{\varepsilon}\right) \leqslant \inf \widetilde{H}_{\mathscr{\mathcal { F }}} \widetilde{\mathcal{F}}_{\varepsilon, h_{\mathrm{ex}}}+o(1)$.

In Section 5.2 we apply a vortex ball construction of Sandier-Serfaty (Proposition 5.5) and we follow the strategy of Sandier-Serfaty developed in [15] to prove that the vorticity of a reasonable configuration is bounded (see Theorem 5.6).

Once the bound on the vorticity yields, we adapt a result of Serfaty [17] which gives a decomposition of $\widetilde{\mathcal{F}}_{\varepsilon, h_{\text {ex }}}\left(v_{\varepsilon}, A_{\varepsilon}\right)$ in term of $F_{\varepsilon}\left(v_{\varepsilon}\right)$ and the location of the vorticity defects (Proposition 5.7).

The decomposition obtained in Proposition 5.7 allows to focus the study on the energy $F_{\varepsilon}$ which ignores the magnetic field. From this point on, the study of a configuration $\left(v_{\varepsilon}, A_{\varepsilon}\right)$ is done for a major part via classical results based on the case without magnetic field (as in [4]). To this end we adapt to our case some standard estimates ignoring the magnetic field, in particular the crucial notion of Renormalized energies is presented in Section 6 .

With these preliminary results, in Section 7 , for $d \in \mathbb{N}^{*}$, we construct competitors $\left(v_{\varepsilon}, A_{\varepsilon}\right) \in \mathscr{H}$ with $d$ quantized vorticity defects and then we get a sharp upper bound (see Proposition 7.3):

$$
\inf _{\mathscr{H}} \mathcal{F}_{\varepsilon, h_{\mathrm{ex}}} \leqslant h_{\mathrm{ex}}^{2} \mathbf{J}_{\mathbf{0}}+d M_{\Omega}\left[-h_{\mathrm{ex}}+H_{c_{1}}^{0}\right]+\mathscr{L}_{1}(d) \ln h_{\mathrm{ex}}+\mathscr{L}_{2}(d)+o(1) .
$$

Here $\mathbf{J}_{\mathbf{0}}$ and $M_{\Omega}$ are independent of $\varepsilon$ and $d, \mathscr{L}_{1}(d)$ and $\mathscr{L}_{2}(d)$ are independent of $\varepsilon$ and $H_{c_{1}}^{0}$ is the leading term in the expression of the first critical field.

With the above upper bound for the minimal energy, the heart of the work consists in getting lower bounds for quasi-minimizers. Before getting such lowers bounds we adapt to our case some tools in Section 8: an $\eta$-ellipticity result is proved (Proposition 8.1), a construction of ad-hoc bad-discs is done (Proposition 8.3) and the strong effect of the dilution is expressed by various results in Section 8.3.

In Section 9 we begin the proof of the theorems. The part of Theorem 3.3 related with Theorem 1.2 is a direct consequence of Propositions 9.3, 9.4, 9.6 and 9.7 (and also Corollary 10.4).

The part of Theorem 3.3 related with Theorem 1.3 is given by Corollary 10.1 and Proposition 7.3. 
The part of Theorem 3.3 related with Theorem 1.4 is a direct consequence of Corollary 10.4 and Propositions 10.7 and 10.8 .

We end this section by giving some explanations on the technical hypotheses (1.2) and (1.4).

Hypothesis (1.2) consists in two estimates: $\lambda^{1 / 4}|\ln \varepsilon| \rightarrow 0$ and $|\ln (\lambda \delta)|=$ $\mathcal{O}(\ln |\ln \varepsilon|)$. The estimate " $\lambda^{1 / 4}|\ln \varepsilon| \rightarrow 0$ " is essentially used in the cleaning step (Section 5.1) in conjunction with Proposition 5.3. Without this estimate the perturbative argument is no longer valid.

The estimate " $|\ln (\lambda \delta)|=\mathcal{O}(\ln |\ln \varepsilon|)$ " is frequently used in this work (the same hypothesis was used in [6]). The major use of this hypothesis is to apply Theorem 5.6 which is fundamental in the study. On the one hand, Hypothesis (1.3) is crucial in the strategy to prove Theorem 5.6. On the other hand, vorticity defects appear (see Corollary 10.4) for $h_{\mathrm{ex}} \geqslant H_{c_{1}}+o(1)$ where

$$
H_{c_{1}}=\frac{b^{2}|\ln \varepsilon|+\left(1-b^{2}\right)|\ln (\lambda \delta)|}{2\left\|\xi_{0}\right\|_{L^{\infty}(\Omega)}}+\mathcal{O}(1) .
$$

Consequently, if we want to deal with vorticity defects and with the (fundamental) conclusion of Theorem 5.6 then we need assume " $|\ln (\lambda \delta)|=$ $\mathcal{O}(\ln |\ln \varepsilon|) "$.

Hypothesis (1.4) is often used in a weaker form $\left(\delta h_{\mathrm{ex}} \rightarrow 0\right)$. Estimate $(1.4)$ is crucial in Proposition 9.6 to get macroscopic and mesoscopic informations on the location of the vorticity defects.

\section{Some preliminaries}

\subsection{Energetic cleaning}

In order to do the cleaning step, we have to get some estimates. Our goal is to study quasi-minimizer of $\mathcal{F}_{\varepsilon, h_{\mathrm{ex}}}$. To keep a simple presentation, we write $\mathcal{F}$ instead of $\mathcal{F}_{\varepsilon, h_{\mathrm{ex}}}$ and $F$ instead of $F_{\varepsilon}$ when there is no ambiguity.

From (3.2), (3.3) and classical elliptic regularity arguments we have the following proposition.

Proposition 5.1. - Let $\left\{\left(v_{\varepsilon}, A_{\varepsilon}\right) \mid 0<\varepsilon<1\right\} \subset \mathscr{H}$ be a family of configurations in the Coulomb gauge. Then there is $\xi_{\varepsilon} \in H_{0}^{1} \cap H^{2}(\Omega, \mathbb{R})$ s.t. $A_{\varepsilon}=\nabla^{\perp} \xi_{\varepsilon}$. Moreover, if for some $h_{\mathrm{ex}}=h_{\mathrm{ex}}(\varepsilon)$ we have

$$
\mathcal{F}\left(v_{\varepsilon}, A_{\varepsilon}\right)=\mathcal{O}\left(h_{\mathrm{ex}}^{2}\right),
$$


then there exists $C$ (independent of $\varepsilon$ ) s.t.

$$
\left\|\xi_{\varepsilon}\right\|_{H^{2}(\Omega)} \leqslant C h_{\mathrm{ex}} .
$$

Consequently, for $p \in[1, \infty)$, there exists $C_{p}>1$ (independent of $\varepsilon$ ) s.t.

$$
\left\|\nabla \xi_{\varepsilon}\right\|_{L^{p}(\Omega)}=\left\|A_{\varepsilon}\right\|_{L^{p}(\Omega)} \leqslant C_{p} h_{\mathrm{ex}} .
$$

Moreover, up to increasing the value of $C>1$ (independently of $\varepsilon$ ), we have

$$
\left\|\nabla v_{\varepsilon}\right\|_{L^{2}(\Omega)} \leqslant C h_{\mathrm{ex}} .
$$

And if $\operatorname{curl}\left(A_{\varepsilon}\right) \in H^{1}(\Omega)$ then

$$
\left\|\xi_{\varepsilon}\right\|_{H^{3}(\Omega)} \leqslant C\left\|\operatorname{curl}\left(A_{\varepsilon}\right)\right\|_{H^{1}(\Omega)} .
$$

In particular, for further use, note that if $\operatorname{curl}\left(A_{\varepsilon}\right) \in H^{1}(\Omega)$ then $\xi_{\varepsilon} \in H_{0}^{1} \cap$ $H^{2} \cap W^{1, \infty}(\Omega)$ and

$$
\left\|\nabla \xi_{\varepsilon}\right\|_{L^{\infty}(\Omega)} \leqslant C\left\|\operatorname{curl}\left(A_{\varepsilon}\right)\right\|_{H^{1}(\Omega)} .
$$

In order to do the cleaning step we need to underline the fact that $U_{\varepsilon}$ may be seen as a regularization of $a_{\varepsilon}$ in $W^{1, \infty}$ with estimates that become bad when approaching $\partial \omega_{\varepsilon}$.

Proposition 5.2. - There exist $C_{b}, s_{b}>0$ depending only on $b$ and $\Omega$ s.t. for $\varepsilon, r>0$ we have:

$$
\begin{gathered}
\left\|\nabla U_{\varepsilon}\right\|_{L^{\infty}(\Omega)} \leqslant \frac{C_{b}}{\varepsilon}, \\
\left|U_{\varepsilon}-a_{\varepsilon}\right| \leqslant C_{b} \mathrm{e}^{-\frac{s_{b} r}{\varepsilon}} \text { in }\left\{x \in \Omega \mid \operatorname{dist}\left(x, \partial \omega_{\varepsilon}\right) \geqslant r\right\}, \\
\left|\nabla U_{\varepsilon}\right| \leqslant \frac{C_{b} \mathrm{e}^{-\frac{s_{b} r}{\varepsilon}}}{\varepsilon} \text { in }\left\{x \in \Omega \mid \operatorname{dist}\left(x, \partial \omega_{\varepsilon}\right) \geqslant r\right\} .
\end{gathered}
$$

Proof. - Estimate (5.7) is a consequence of Lemma 3.2. The proof of (5.8) is the same than Proposition 2 in [9]. Estimate (5.9) is proved in Appendix A.

Since the 2-dimensional Hausdorff measure of $\omega_{\varepsilon}$ satisfies $\mathcal{H}^{2}\left(\omega_{\varepsilon}\right)=\mathcal{O}\left(\lambda^{2}\right)$, from (5.8), for $p \in[1, \infty[$, we have the following crucial estimate

$$
\left\|U_{\varepsilon}^{2}-1\right\|_{L^{p}(\Omega)}=\mathcal{O}\left(\lambda^{2 / p}\right) .
$$

We are now in position to do the cleaning step. We assume that $\left\{\left(v_{\varepsilon}, A_{\varepsilon}\right) \mid\right.$ $0<\varepsilon<1\} \subset \mathscr{H}$ is a family of configuration in the Coulomb gauge which satisfies (5.1). We denote $\alpha_{\varepsilon}=U_{\varepsilon}^{2}$ and $\rho_{\varepsilon}=\left|v_{\varepsilon}\right|$. From direct computations, by splitting the integrals with the identity $\alpha_{\varepsilon}=\left(\alpha_{\varepsilon}-1\right)+1$ and using $\left(1-\rho_{\varepsilon}\right)^{4} \leqslant\left(1-\rho_{\varepsilon}^{2}\right)^{2}$, we have the existence of $C \geqslant 1$ (independent of $\varepsilon$ ) s.t.

$$
\left|\int_{\Omega} \alpha_{\varepsilon}\left(v_{\varepsilon} \wedge \nabla v_{\varepsilon}\right) \cdot A_{\varepsilon}-\int_{\Omega}\left(v_{\varepsilon} \wedge \nabla v_{\varepsilon}\right) \cdot A_{\varepsilon}\right| \leqslant C \sqrt{\lambda} h_{\mathrm{ex}}^{2}
$$


and

$$
\left.\left|\int_{\Omega} \alpha_{\varepsilon} \rho_{\varepsilon}^{2}\right| A_{\varepsilon}\right|^{2}-\int_{\Omega}\left|A_{\varepsilon}\right|^{2} \mid \leqslant C h_{\mathrm{ex}}^{2}\left(\varepsilon h_{\mathrm{ex}}+\lambda\right) .
$$

By combining (5.11) and (5.12) we immediately get the following proposition.

Proposition 5.3. - If $\left(v_{\varepsilon}, A_{\varepsilon}\right)$ is in the Coulomb gauge and satisfies (5.1) then

$$
\left|\widetilde{\mathcal{F}}\left(v_{\varepsilon}, A_{\varepsilon}\right)-\mathcal{F}\left(v_{\varepsilon}, A_{\varepsilon}\right)\right| \leqslant C h_{\mathrm{ex}}^{2}\left(\varepsilon h_{\mathrm{ex}}+\sqrt{\lambda}\right)
$$

with $C$ which is independent of $\varepsilon$ and

$$
\begin{aligned}
\widetilde{\mathcal{F}}(v, A) & =\widetilde{\mathcal{F}}_{\varepsilon, h_{\mathrm{ex}}}(v, A) \\
& :=F(v)+\frac{1}{2} \int_{\Omega}-2(v \wedge \nabla v) \cdot A+|A|^{2}+\left|\operatorname{curl}(A)-h_{\mathrm{ex}}\right|^{2} .
\end{aligned}
$$

Remark 5.4.

(1) One may claim that $\widetilde{\mathcal{F}}$ is not gauge invariant if $\alpha_{\varepsilon} \not \equiv 1$.

(2) Note that if $\lambda^{1 / 4}|\ln \varepsilon| \rightarrow 0$ and if $h_{\mathrm{ex}}=\mathcal{O}(|\ln \varepsilon|)$ then for $\left(v_{\varepsilon}, A_{\varepsilon}\right) \in$ $\mathscr{H}$ which is in the Coulomb gauge and satisfies (5.1) we have $\widetilde{\mathcal{F}}\left(v_{\varepsilon}, A_{\varepsilon}\right)-\mathcal{F}\left(v_{\varepsilon}, A_{\varepsilon}\right)=o(1)$ without any assumption on $\delta \in(0,1)$.

\subsection{Bound on the vorticity and energetic decomposition}

By applying Proposition 1 in [15] with $U_{\varepsilon} \geqslant b$ we immediately get the following proposition which does not need any assumption for $\lambda, \delta \in(0,1)$.

Proposition 5.5. - Assume $h_{\mathrm{ex}} \leqslant C_{0}|\ln \varepsilon|$ with $C_{0} \geqslant 1$ which is independent of $\varepsilon$. Let $\left\{\left(v_{\varepsilon}, A_{\varepsilon}\right) \mid 0<\varepsilon<1\right\}$ be a family s.t. $\mathcal{F}\left(v_{\varepsilon}, A_{\varepsilon}\right) \leqslant C_{0}|\ln \varepsilon|^{2}$.

Then there exist $C, \varepsilon_{0}>0$ (depending only on $\Omega, b$ and $C_{0}$ ) s.t. for $\varepsilon<\varepsilon_{0}$ we have either $\left|v_{\varepsilon}\right| \geqslant 1-|\ln \varepsilon|^{-2}$ in $\Omega$ or there exists a finite family of disjoint disks $\left\{B_{i} \mid i \in \mathcal{J}\right\}$ with $\mathcal{J} \subset \mathbb{N}^{*}(\mathcal{J}$ depends on $\varepsilon)$ and $B_{i}:=B\left(a_{i}, r_{i}\right)$ satisfying:

(1) $\left\{\left|v_{\varepsilon}\right|<1-|\ln \varepsilon|^{-2}\right\} \subset \bigcup B_{i}$

(2) $\sum r_{i}<|\ln \varepsilon|^{-10}$,

(3) writing $h_{\varepsilon}=\operatorname{curl}\left(A_{\varepsilon}\right), \rho_{\varepsilon}=\left|v_{\varepsilon}\right|$ and $v_{\varepsilon}=\rho_{\varepsilon} \mathrm{e}^{i \varphi_{\varepsilon}} \quad\left(\varphi_{\varepsilon}\right.$ is locally defined) we have

$\frac{1}{2} \int_{B_{i}} \rho^{2}\left|\nabla \varphi_{\varepsilon}-A_{\varepsilon}\right|^{2}+\left|h_{\varepsilon}-h_{\mathrm{ex}}\right|^{2} \geqslant \pi\left|d_{i}\right|(|\ln \varepsilon|-C \ln |\ln \varepsilon|)$,

with $d_{i}=\operatorname{deg}_{\partial B_{i}}(v)$ if $B_{i} \subset \Omega$ and 0 otherwise. 
By following the argument of Sandier and Serfaty [15], we get the main result of this section.

TheOrem 5.6. - Assume that $\lambda, \delta$ satisfy (1.2) and $\delta^{2}|\ln \varepsilon| \leqslant 1$. Assume also Hypothesis (1.3) holds for $h_{\mathrm{ex}}$ with some $K \geqslant 1$.

Then there exist $\varepsilon_{K}>0$ and $\mathcal{M}_{K} \geqslant 1$ (independent of $\varepsilon$ ) s.t. if $\left\{\left(v_{\varepsilon}, A_{\varepsilon}\right) \mid\right.$ $0<\varepsilon<1\} \subset \mathscr{H}$ is a family in the Coulomb gauge satisfying $\mathcal{F}\left(v_{\varepsilon}, A_{\varepsilon}\right) \leqslant$ $\inf _{\mathscr{H}} \mathcal{F}+K \ln |\ln \varepsilon|$ then for $0<\varepsilon<\varepsilon_{K}$ we have

$$
\frac{1}{2} \int_{\Omega}\left|\nabla v_{\varepsilon}\right|^{2}+\frac{1}{2 \varepsilon^{2}}\left(1-\left|v_{\varepsilon}\right|^{2}\right)^{2} \leqslant \mathcal{M}_{K}|\ln \varepsilon| .
$$

Moreover, if $\left|v_{\varepsilon}\right| \ngtr 1-|\ln \varepsilon|^{-2}$ in $\Omega$, then letting $\left\{B_{i} \mid i \in \mathcal{J}\right\}$ be a family of disks given by Proposition 5.5, for $0<\varepsilon<\varepsilon_{K}$, we have $d_{i} \geqslant 0$ for all $i \in \mathcal{J}$ and there is $s_{0}>0$ (depending only on $\Omega$ ) s.t. if $i \in \mathcal{J}$ is s.t. $d_{i} \neq 0$ then $\operatorname{dist}\left(B_{i}, \Lambda\right) \leqslant \mathcal{M}_{K}|\ln \varepsilon|^{-s_{0}}$.

The proof of this theorem is postponed in Appendix B.

We let

$$
\mathbf{J}_{\mathbf{0}}:=\widetilde{\mathcal{F}}_{1,1}\left(1, \nabla^{\perp} \xi_{0}\right)=\frac{\widetilde{\mathcal{F}}_{\varepsilon, h_{\mathrm{ex}}}\left(1, h_{\mathrm{ex}} \nabla^{\perp} \xi_{0}\right)}{h_{\mathrm{ex}}^{2}} .
$$

Note that if $\left\{\left(v_{\varepsilon}, A_{\varepsilon}\right) \mid 0<\varepsilon<1\right\}$ is a family of quasi-minimizers then

$$
\mathcal{F}_{\varepsilon, h_{\mathrm{ex}}}\left(v_{\varepsilon}, A_{\varepsilon}\right) \leqslant \mathcal{F}_{\varepsilon, h_{\mathrm{ex}}}\left(1, \nabla^{\perp} \xi_{0}\right)+o(1)=h_{\mathrm{ex}}^{2} \mathbf{J}_{\mathbf{0}}+o(1)=\mathcal{O}\left(h_{\mathrm{ex}}^{2}\right) .
$$

The discs given by Proposition 5.5 are "too large" for our strategy. Indeed one of the main argument is a construction of bad discs in the spirit of [4] which links $x_{\varepsilon} \in\left\{\left|v_{\varepsilon}\right| \leqslant 1 / 2\right\}$ with the energetic cost in a ball $B\left(x_{\varepsilon}, \varepsilon^{\mu}\right)$ with small $\mu>0$. Namely if $x_{\varepsilon} \in\left\{\left|v_{\varepsilon}\right|<1-|\ln \varepsilon|^{-2}\right\} \subset \bigcup B_{i}$ then the energetic cost in a ball $B\left(x_{\varepsilon}, \varepsilon^{\mu}\right)$ is not sufficiently large comparing to our error term.

In the next proposition we present the good framework of vortex balls required in the study. The first step in the study is an energetic decomposition valid under some assumptions (no assumption on $\delta \in(0,1)$ is required).

Proposition 5.7. - Let $C_{0}>1,\left(v_{\varepsilon}\right)_{0<\varepsilon<1} \subset H^{1}(\Omega, \mathbb{C})$ and $h_{\mathrm{ex}}>0$ be s.t.

$$
F\left(v_{\varepsilon}\right) \leqslant C_{0}|\ln \varepsilon|^{2}, h_{\mathrm{ex}} \leqslant C_{0}|\ln \varepsilon| .
$$

Assume furthermore that $\lambda^{1 / 4}|\ln \varepsilon| \rightarrow 0$ and, for $\varepsilon \in(0,1)$, either $\left|v_{\varepsilon}\right|>$ $1 / 2$ in $\Omega$ or $v_{\varepsilon}$ admits a family of valued disks $\left\{\left(B\left(a_{i}, r_{i}\right), d_{i}\right) \mid i \in \mathcal{J}\right\}(\mathcal{J}$ is finite) s.t.:

- the disks $B_{i}=B\left(a_{i}, r_{i}\right)$ are pairwise disjoint

- $\left\{\left|v_{\varepsilon}\right| \leqslant 1 / 2\right\} \subset \bigcup_{i \in \mathcal{J}} B_{i}$

- $\sum_{i \in \mathcal{J}} r_{i}<|\ln \varepsilon|^{-10}$ 
- For $i \in \mathcal{J}$, letting

$$
d_{i}= \begin{cases}\operatorname{deg}_{\partial B_{i}}(v) & \text { if } B_{i} \subset \Omega \\ 0 & \text { otherwise, }\end{cases}
$$

we assume $\sum_{i \in \mathcal{J}}\left|d_{i}\right| \leqslant C_{0}$.

Then, if $\left(\xi_{\varepsilon}\right)_{\varepsilon} \subset H_{0}^{1} \cap H^{2} \cap W^{1, \infty}(\Omega, \mathbb{R})$ is s.t.

$$
\left\|\nabla \xi_{\varepsilon}\right\|_{L^{\infty}(\Omega)} \leqslant C_{0}|\ln \varepsilon|,
$$

writing $\zeta_{\varepsilon}:=\xi_{\varepsilon}-h_{\mathrm{ex}} \xi_{0}$ we have in the case $\left|v_{\varepsilon}\right| \ngtr 1 / 2$ in $\Omega$ :

$$
\mathcal{F}\left(v_{\varepsilon}, \nabla^{\perp} \xi_{\varepsilon}\right)-h_{\mathrm{ex}}^{2} \mathbf{J}_{\mathbf{0}}=F\left(v_{\varepsilon}\right)+2 \pi h_{\mathrm{ex}} \sum_{i \in \mathcal{J}} d_{i} \xi_{0}\left(a_{i}\right)+\widetilde{V}_{(\mathbf{a}, \mathbf{d})}\left(\zeta_{\varepsilon}\right)+o(1)
$$

where for $\zeta \in H_{0}^{1} \cap H^{2}(\Omega)$ we denoted

$$
\widetilde{V}_{(\mathbf{a}, \mathbf{d})}(\zeta):=2 \pi \sum_{i \in \mathcal{J}} d_{i} \zeta\left(a_{i}\right)+\frac{1}{2} \int_{\Omega}(\Delta \zeta)^{2}+|\nabla \zeta|^{2} .
$$

And if $|v|>1 / 2$ in $\Omega$ then

$$
\mathcal{F}\left(v_{\varepsilon}, \nabla^{\perp} \xi_{\varepsilon}\right)-h_{\mathrm{ex}}^{2} \mathbf{J}_{\mathbf{0}}=F\left(v_{\varepsilon}\right)+\frac{1}{2} \int_{\Omega}\left(\Delta \zeta_{\varepsilon}\right)^{2}+\left|\nabla \zeta_{\varepsilon}\right|^{2}+o(1)
$$

The proof of Proposition 5.7 is an adaptation of an argument of Serfaty [17, Section 4]. The proof is presented Appendix C

Before going further, we state a result which will be useful in this article and whose proof is left to the reader.

LEMmA 5.8. - For $v \in H^{1}(\Omega, \mathbb{C}), 0<\varepsilon<1$ and $h_{\mathrm{ex}}>0$, there exists a unique potential $A_{v, \varepsilon, h_{\mathrm{ex}}}=A_{v} \in H^{1}\left(\Omega, \mathbb{R}^{2}\right)$ s.t. $\left(v, A_{v}\right)$ is in the Coulomb gauge and satisfies

$$
\begin{cases}-\nabla^{\perp} \operatorname{curl}\left(A_{v}\right)=\alpha(\imath v) \cdot\left(\nabla v-\imath A_{v} v\right) & \text { in } \Omega \\ \operatorname{curl}\left(A_{v}\right)=h_{\mathrm{ex}} & \text { on } \partial \Omega .\end{cases}
$$

Moreover $A_{v}$ is the unique solution of the minimization problem

$$
\inf _{\text {A satisfies (3.1) }} \mathcal{F}_{\varepsilon, h_{\mathrm{ex}}}(v, A)
$$

and from (3.3) and (3.4) we have $A_{v}=\nabla^{\perp} \xi_{v}$ with $\xi_{v} \in H_{0}^{1} \cap H^{2} \cap W^{1, \infty}(\Omega, \mathbb{R})$.

Remark 5.9. - Assume $\lambda, \delta$ satisfy (1.2), $\delta^{2}|\ln \varepsilon| \leqslant 1$ and Hypothesis (1.3) holds. Consider $\left\{\left(v_{\varepsilon}, A_{\varepsilon}\right) \mid 0<\varepsilon<1\right\} \subset \mathscr{H}$ a family in the Coulomb gauge satisfying $\mathcal{F}\left(v_{\varepsilon}, A_{\varepsilon}\right) \leqslant \inf _{\mathscr{H}} \mathcal{F}+\mathcal{O}(\ln |\ln \varepsilon|)$.

- From Theorem 5.6, either $\left|v_{\varepsilon}\right|>1-|\ln \varepsilon|^{-2}$ in $\Omega$ or the family of disjoint disks given by Proposition 5.5 satisfies the properties of the family of discs used in Proposition 5.7. 
- Let $A_{v_{\varepsilon}}=\nabla^{\perp} \xi_{v_{\varepsilon}} \in H^{1}\left(\Omega, \mathbb{R}^{2}\right)$ be given by Lemma 5.8. Then with (3.3) and (5.22) we have $A_{v_{\varepsilon}} \in L^{\infty}(\Omega)$ and $\left\|A_{v_{\varepsilon}}\right\|_{L^{\infty}(\Omega)} \leqslant$ $C|\ln \varepsilon|$ where $C$ depends only on $\Omega$.

As noted by Serfaty [17], with the help of the decomposition given by Proposition 5.7, we may prove that $h_{\mathrm{ex}}^{2} \mathbf{J}_{\mathbf{0}}$ is almost the minimal energy of a vortexless configuration.

Corollary 5.10. - Let

$$
\mathscr{H}^{0}:=\left\{\left(\rho \mathrm{e}^{\imath \varphi}, A\right) \mid \rho \in H^{1}(\Omega,[0, \infty)), \varphi \in H^{1}(\Omega, \mathbb{R}) \text { and } A \in H^{1}\left(\Omega, \mathbb{R}^{2}\right)\right\} .
$$

Note that $\mathscr{H}^{0}$ is gauge invariant. Assume $\lambda^{1 / 4}|\ln \varepsilon| \rightarrow 0$.

(1) Let $\varepsilon=\varepsilon_{n} \downarrow 0$. Assume $h_{\mathrm{ex}}=\mathcal{O}(|\ln \varepsilon|)$ and for each $\varepsilon$ let $\left(v_{\varepsilon}, \nabla^{\perp} \xi_{\varepsilon}\right) \in$ $\mathscr{H}^{0}$ be s.t. $\xi_{\varepsilon} \in H_{0}^{1} \cap H^{2} \cap W^{1, \infty}(\Omega, \mathbb{R})$ with $\left\|\nabla \xi_{\varepsilon}\right\|_{L^{\infty}(\Omega)}=\mathcal{O}(|\ln \varepsilon|)$. Writing $\zeta_{\varepsilon}:=\xi_{\varepsilon}-h_{\mathrm{ex}} \xi_{0}$ we have:

$$
\mathcal{F}\left(v_{\varepsilon}, \nabla^{\perp} \xi_{\varepsilon}\right)=h_{\mathrm{ex}}^{2} \mathbf{J}_{\mathbf{0}}+F\left(v_{\varepsilon}\right)+\frac{1}{2} \int_{\Omega}\left(\Delta \zeta_{\varepsilon}\right)^{2}+\left|\nabla \zeta_{\varepsilon}\right|^{2}+o(1) .
$$

Thus, if $\mathcal{F}\left(v_{\varepsilon}, \nabla^{\perp} \xi_{\varepsilon}\right) \leqslant h_{\mathrm{ex}}^{2} \mathbf{J}_{\mathbf{0}}+o(1)$ then $\zeta_{\varepsilon} \rightarrow 0$ in $H^{2}(\Omega),\left|v_{\varepsilon}\right| \rightarrow 1$ in $H^{1}(\Omega)$ and, up to a subsequence, there exists $\mathrm{v} \in \mathbb{S}^{1}$ s.t. $v_{\varepsilon} \rightarrow \mathrm{v}$ in $H^{1}(\Omega)$.

(2) We have $\inf _{\mathscr{H}^{\circ}} \mathcal{F}=h_{\text {ex }}^{2} \mathbf{J}_{\mathbf{0}}+o(1)$.

Proof. - We prove the first assertion. Estimate (5.24) is a direct consequence of Proposition 5.7.

For sake of simplicity of the presentation we drop the subscript $\varepsilon$. If $\mathcal{F}\left(v, \nabla^{\perp} \xi\right) \leqslant h_{\mathrm{ex}}^{2} \mathbf{J}_{\mathbf{0}}+o(1)$, then $F(v)+\|\zeta\|_{H^{2}(\Omega)}=o(1)$ and then $\zeta \rightarrow 0$ in $H^{2}(\Omega),|v| \rightarrow 1$ in $H^{1}(\Omega)$. Moreover $\|\nabla v\|_{L^{2}(\Omega)}=o(1)$ and $\|v\|_{L^{2}(\Omega)}=\mathcal{O}(1)$. This clearly implies the remaining part of the assertion.

We prove the second assertion. We first claim, by the definition of $\mathbf{J}_{\mathbf{0}}$, that using the configuration $\left(1, h_{\text {ex }} \nabla^{\perp} \xi_{0}\right) \in \mathscr{H}^{0}$ we have inf $\mathscr{H}^{0} \mathcal{F} \leqslant h_{\text {ex }}^{2} \mathbf{J}_{\mathbf{0}}+o(1)$.

By the gauge invariance of $\mathscr{H}^{0}$ we may consider a family of quasiminimizer $\left\{\left(v_{\varepsilon}, A_{\varepsilon}\right) \mid 0<\varepsilon<1\right\} \subset \mathscr{H}^{0}$ which is in the Coulomb gauge. We write $\left(v_{\varepsilon}, A_{\varepsilon}\right)=(v, A)$. Let $(\widetilde{v}, \widetilde{A}) \in \mathscr{H}^{0}$ be defined by $\widetilde{v}=\underline{v}$ and $\widetilde{A}$ is the unique solution of (5.23) associated to $\widetilde{v}$. $o(1)$.

By direct calculations we have: $\mathcal{F}(\widetilde{v}, \widetilde{A}) \leqslant \mathcal{F}(\widetilde{v}, A) \leqslant \mathcal{F}(v, A) \leqslant h_{\mathrm{ex}}^{2} \mathbf{J}_{\mathbf{0}}+$

Moreover, by denoting $h:=\operatorname{curl}(\widetilde{A})$, we have $\nabla h=\alpha \widetilde{v} \wedge\left(\nabla^{\perp} \widetilde{v}-\widetilde{A}^{\perp} \widetilde{v}\right)$ in $\Omega$ and $h=h_{\text {ex }}$ on $\partial \Omega$. Then $\|h\|_{H^{1}(\Omega)}=\mathcal{O}(|\ln \varepsilon|)$ and using (5.5) we get $\|\widetilde{A}\|_{H^{2}(\Omega)}=\mathcal{O}(|\ln \varepsilon|)$. 
We are then able to apply the first assertion to get $\mathcal{F}(\widetilde{v}, \widetilde{A}) \geqslant h_{\mathrm{ex}}^{2} \mathbf{J}_{\mathbf{0}}+$ $o(1)$.

\subsection{Pseudo vortex structure}

We assume $\lambda^{1 / 4}|\ln \varepsilon| \rightarrow 0$. Let $\left\{\left(v_{\varepsilon}, A_{\varepsilon}\right) \mid 0<\varepsilon<1\right\} \subset \mathscr{H}$ be a family of configurations in the Coulomb gauge satisfying (5.17). We assume that $\left|v_{\varepsilon}\right| \ngtr$ $1 / 2$ in $\Omega$ and that there exists $\left\{\left(B\left(a_{i}, r_{i}\right), d_{i}\right) \mid i \in \mathcal{J}\right\}$ as in Proposition 5.7. Then Proposition 5.7 gives a decomposition of $\mathcal{F}(v, A)$. Except in the crucial hypothesis $\sum r_{i}<|\ln \varepsilon|^{-10}$, the radii $r_{i}$ do not play any role as well as the disks " $B\left(a_{i}, r_{i}\right)$ " associated to a zero degree. We thus introduce an ad-hoc notion of pseudo vortex.

DEFINITION 5.11. - We assume that we have either $\varepsilon=\varepsilon_{n} \downarrow 0$ or $0<\varepsilon<1$. We consider $\left(v_{\varepsilon}\right)_{\varepsilon} \subset H^{1}(\Omega, \mathbb{C}),\left(h_{\mathrm{ex}}\right)_{\varepsilon} \subset(1, \infty)$ satisfying $(5.17)$.

Let $\left\{B_{i}=B\left(a_{i}, r_{i}\right) \mid i \in \mathcal{J}\right\}$ be a family of disks as in Proposition 5.7 and let $d_{i}=d_{i}^{(\varepsilon)} \in \mathbb{Z}$ be the associated "degrees" defined in Proposition 5.7. We denote $\mathcal{J}^{\prime}=\mathcal{J}_{\varepsilon}^{\prime}:=\left\{i \in \mathcal{J} \mid d_{i} \neq 0\right\}$ (note that we have $\operatorname{Card}\left(\mathcal{J}_{\varepsilon}^{\prime}\right) \leqslant \sum\left|d_{i}\right|=$ $\mathcal{O}(1))$.

If $\mathcal{J}^{\prime} \neq \emptyset$, then we say that $\{(\mathbf{a}, \mathbf{d})\}=\left\{\left(a_{i}, d_{i}\right) \mid i \in \mathcal{J}^{\prime}\right\}$ is a set of pseudo vortices of $v_{\varepsilon}$.

For a fixed configuration $(\mathbf{a}, \mathbf{d})$ of pseudo vortices, Serfaty studied in [17] the minimization problem of $\widetilde{V}_{(\mathbf{a}, \mathbf{d})}$ (defined in (5.20)). We have the following result ([17, Proposition 4.2]).

Proposition 5.12. - Let $(\mathbf{a}, \mathbf{d})=\left\{\left(a_{i}, d_{i}\right) \mid i \in \mathcal{J}^{\prime}\right\} \subset \Omega \times \mathbb{Z}^{*}$ be $a$ configuration s.t. $1 \leqslant \operatorname{Card}\left(\mathcal{J}^{\prime}\right)<\infty$ and $a_{i} \neq a_{j}$ for $i \neq j$. Then $\widetilde{V}_{(\mathbf{a}, \mathbf{d})}(\zeta)$ is minimal for $\zeta=\zeta_{(\mathbf{a}, \mathbf{d})}$ which satisfies

$$
\begin{cases}-\Delta^{2} \zeta_{(\mathbf{a}, \mathbf{d})}+\Delta \zeta_{(\mathbf{a}, \mathbf{d})}=2 \pi \sum_{i \in \mathcal{J}^{\prime}} d_{i} \delta_{a_{i}} & \text { in } \Omega \\ \zeta_{(\mathbf{a}, \mathbf{d})}=\Delta \zeta_{(\mathbf{a}, \mathbf{d})}=0 & \text { on } \partial \Omega .\end{cases}
$$

(Here $\delta_{a}$ is the Dirac mass at $a \in \mathbb{R}^{2}$.)

$$
\text { And we have } \widetilde{V}\left[\zeta_{(\mathbf{a}, \mathbf{d})}\right]=\pi \sum_{i \in \mathcal{J}^{\prime}} d_{i} \zeta_{(\mathbf{a}, \mathbf{d})}\left(a_{i}\right) \text {. }
$$

In order to prove the above proposition, Serfaty introduced for $a \in \Omega$ the function $\zeta^{a} \in H_{0}^{1} \cap H^{2}(\Omega)$ which is the unique solution of

$$
\begin{cases}-\Delta^{2} \zeta^{a}+\Delta \zeta^{a}=2 \pi \delta_{a} & \text { in } \Omega \\ \zeta^{a}=\Delta \zeta^{a}=0 & \text { on } \partial \Omega .\end{cases}
$$


In particular we have $\zeta^{a} \leqslant 0$ in $\Omega$. It is easy to see that $\zeta_{(\mathbf{a}, \mathbf{d})}=\sum_{i \in \mathcal{J}^{\prime}} d_{i} \zeta^{a_{i}}$ is the unique solution of (5.25).

Lemma 4.6 in [17] gives important properties related with $\zeta^{a}$ and $\zeta_{(\mathbf{a}, \mathbf{d})}$ :

Proposition 5.13. - For $s \in(0,1)$, there exists $C_{s}>0$ s.t. for $a, b \in \Omega$

$$
\left\|\zeta^{a}\right\|_{L^{\infty}(\Omega)} \leqslant C_{s} \operatorname{dist}(a, \partial \Omega)^{s}
$$

and

$$
\left\|\zeta^{a}-\zeta^{b}\right\|_{H^{2}(\Omega)} \leqslant C_{s}|a-b|^{s} .
$$

Consequently there exists $C>0$ depending only on $\Omega$ s.t., if $\zeta_{(\mathbf{a}, \mathbf{d})}$ is the unique solution of (5.25), then

$$
\left|\widetilde{V}\left[\zeta_{(\mathbf{a}, \mathbf{d})}\right]\right|=\left|\pi \sum_{i, j \in \mathcal{J}^{\prime}} d_{i} d_{j} \zeta^{a_{i}}\left(a_{j}\right)\right| \leqslant C\left(\sum_{i \in \mathcal{J}^{\prime}}\left|d_{i}\right|\right)^{2} .
$$

For a further use we need the following lemma.

Lemma 5.14. - Let $(\mathbf{a}, \mathbf{d})$ be as in Proposition 5.12 then $\zeta_{(\mathbf{a}, \mathbf{d})} \in H_{0}^{1} \cap$ $H^{2} \cap W^{1, \infty}(\Omega, \mathbb{R})$ and there is $C \geqslant 1$ depending only on $\Omega$ s.t.

$$
\left\|\nabla \zeta_{(\mathbf{a}, \mathbf{d})}\right\|_{L^{\infty}(\Omega)} \leqslant \frac{C \sum\left|d_{i}\right|}{\min \operatorname{dist}\left(a_{i}, \partial \Omega\right)} .
$$

Proof. - Let (a,d) be as in Proposition 5.12, with Proposition 5.13 we have $\zeta_{(\mathbf{a}, \mathbf{d})}=\sum d_{i} \zeta^{a_{i}} \in H_{0}^{1} \cap H^{2}$ and $\left\|\zeta_{(\mathbf{a}, \mathbf{d})}\right\|_{H^{2}(\Omega)} \leqslant C \sum_{i}\left|d_{i}\right|$ where $C$ depends only on $\Omega$.

Moreover, from (5.25), we have $\Delta \zeta_{(\mathbf{a}, \mathbf{d})}=\zeta_{(\mathbf{a}, \mathbf{d})}+\sum d_{i} \ln \left|x-a_{i}\right|+R_{(\mathbf{a}, \mathbf{d})}$ where $R_{(\mathbf{a}, \mathbf{d})}$ is the harmonic extension of $\operatorname{tr}_{\partial \Omega}\left(-\sum d_{i} \ln \left|x-a_{i}\right|\right)$ in $\Omega$.

Consequently there exists $C \geqslant 1$ depending only on $\Omega$ s.t.

$$
\left\|\Delta \zeta_{(\mathbf{a}, \mathbf{d})}\right\|_{L^{3}(\Omega)} \leqslant \frac{C \sum\left|d_{i}\right|}{\min \operatorname{dist}\left(a_{i}, \partial \Omega\right)}
$$

and therefore by elliptic regularity and a Sobolev embedding we get the result.

Until now, the only way to get a nice magnetic potential associated to a function $v$ was to consider $A_{v}=A_{v, \varepsilon, \alpha} \in H^{2}\left(\Omega, \mathbb{R}^{2}\right)$, the unique solution of (5.23). The previous results give that, after the cleaning step, we can do asymptotically as well by using a magnetic potential depending on a pseudo vortices structure of $v$ instead of $v$ itself (see Remark 5.16).

Definition 5.15. - Let $N \geqslant 1$ and $(\mathbf{a}, \mathbf{d}) \in\left(\Omega^{N}\right)^{*} \times\left(\mathbb{Z}^{*}\right)^{N}, h_{\mathrm{ex}}>0$. Then we define $A_{(\mathbf{a}, \mathbf{d})}:=h_{\mathrm{ex}} \nabla^{\perp} \xi_{0}+\nabla^{\perp} \zeta_{(\mathbf{a}, \mathbf{d})}$ where $\zeta_{(\mathbf{a}, \mathbf{d})}$ is the unique solution of (5.25), the potential associated to $(\mathbf{a}, \mathbf{d})$. 
Remark 5.16. - Let $C_{0}>1$ and $\left(v_{\varepsilon}\right)_{0<\varepsilon<1} \subset H^{1}(\Omega, \mathbb{C}), h_{\mathrm{ex}}>0$ satisfying (5.17) be s.t. $\left(v_{\varepsilon}\right)_{0<\varepsilon<1}$ admits a set of pseudo vortices $\left((\mathbf{a}, \mathbf{d})_{\varepsilon}\right)_{0<\varepsilon<1}$ with $\sum\left|d_{i}\right| \leqslant C_{0}$. We write $v$ and $(\mathbf{a}, \mathbf{d})$ instead of $v_{\varepsilon}$ and $(\mathbf{a}, \mathbf{d})_{\varepsilon}$.

Assume min $\operatorname{dist}\left(a_{i}, \partial \Omega\right)>|\ln \varepsilon|^{-1}$ in order to have $\left\|\nabla \zeta_{(\mathbf{a}, \mathbf{d})}\right\|_{L^{\infty}(\Omega)}=$ $\mathcal{O}(|\ln \varepsilon|)$ (with Lemma 5.14) and $\lambda^{1 / 4}|\ln \varepsilon| \rightarrow 0$.

For $0<\varepsilon<1$, let $A_{v} \in H^{1}\left(\Omega, \mathbb{R}^{2}\right)$ be the unique solution of (5.23) and $A_{(\mathbf{a}, \mathbf{d})}$ be defined in Definition 5.15. Then we have $A_{(\mathbf{a}, \mathbf{d})}=\nabla^{\perp} \xi_{(\mathbf{a}, \mathbf{d})}$ and $A_{v}=\nabla^{\perp} \xi_{v}$ where $\xi_{(\mathbf{a}, \mathbf{d})}, \xi_{v} \in H_{0}^{1} \cap H^{2} \cap W^{1, \infty}(\Omega, \mathbb{R})$ satisfy the hypotheses of Proposition 5.7 (here we used (3.3) and (5.22)). Therefore we have the following inequalities

$$
\begin{gathered}
\mathcal{F}(v, 0) \geqslant \mathcal{F}\left(v, A_{v}\right)=\widetilde{\mathcal{F}}\left(v, A_{v}\right)+o(1) \geqslant \widetilde{\mathcal{F}}\left(v, A_{(\mathbf{a}, \mathbf{d})}\right)+o(1), \\
\mathcal{F}\left(v, A_{v}\right) \leqslant \mathcal{F}\left(v, A_{(\mathbf{a}, \mathbf{d})}\right)=\widetilde{\mathcal{F}}\left(v, A_{(\mathbf{a}, \mathbf{d})}\right)+o(1) .
\end{gathered}
$$

In particular we have $\mathcal{F}\left(v, A_{v}\right)=\mathcal{O}\left(|\ln \varepsilon|^{2}\right)$ and $\mathcal{F}\left(v, A_{(\mathbf{a}, \mathbf{d})}\right)=\mathcal{O}\left(|\ln \varepsilon|^{2}\right)$.

\subsection{Cluster of pseudo vortices}

From a standard result for the homogenous case, it is expected that, for a reasonable magnetic field, the asymptotic location of pseudo vortices of a studied configuration is a subset of $\Lambda$. This problem is related to the macroscopic location of the pseudo vortices. To treat this problem we use an ad-hoc notion of cluster of pseudo vortices.

Definition 5.17. - Let $N, \widetilde{N}_{0} \in \mathbb{N}^{*}, \widetilde{N}_{0} \leqslant N,(\mathbf{p}, \mathbf{D}) \in\left(\bar{\Omega}^{\tilde{N}_{0}}\right)^{*} \times \mathbb{Z}^{\tilde{N}_{0}}$, $\varepsilon=\varepsilon_{n} \downarrow 0$ and $(\mathbf{a}, \mathbf{d})_{\varepsilon} \in\left(\Omega^{N}\right)^{*} \times \mathbb{Z}^{N}$ s.t. $\mathbf{D}$ is independent of $\varepsilon$. We say that $(\mathbf{a}, \mathbf{d})_{\varepsilon}$ admits a cluster structure on $(\mathbf{p}, \mathbf{D})$ if

- for $i \in\{1, \ldots, N\}, \lim a_{i}$ exists, $\lim a_{i} \in\left\{p_{1}, \ldots, p_{\tilde{N}_{0}}\right\}$ and we write for $k \in\left\{1, \ldots, \widetilde{N}_{0}\right\}, S_{k}:=\left\{i \in\{1, \ldots, N\} \mid a_{i} \rightarrow p_{k}\right\}$

- for $k \in\left\{1, \ldots, \widetilde{N}_{0}\right\} S_{k} \neq \emptyset$,

- for $k \in\left\{1, \ldots, \widetilde{N}_{0}\right\}, D_{k}=\sum_{i \in S_{k}} d_{i}$.

Remark 5.18. - In this article we will use the notion of cluster structure with $(\mathbf{a}, \mathbf{d})$ as in Proposition 5.7 and $\mathbf{p} \subset \Lambda$.

Proposition 5.19. - Let $N \geqslant 1, \varepsilon=\varepsilon_{n} \downarrow 0,(\mathbf{a}, \mathbf{d})_{\varepsilon} \in\left(\Omega^{N}\right)^{*} \times \mathbb{Z}^{N}$ s.t. $\sum\left|d_{i}\right|$ is bounded independently of $\varepsilon$.

(1) If $\left((\mathbf{a}, \mathbf{d})_{\varepsilon}\right)_{\varepsilon}$ admits a cluster structure on $(\mathbf{p}, \mathbf{D})$ (and then $\mathbf{D}$ is independent of $\varepsilon)$ then $(\mathbf{p}, \mathbf{D})$ is unique. We say that $(\mathbf{p}, \mathbf{D})$ is the cluster of $\left((\mathbf{a}, \mathbf{d})_{\varepsilon}\right)_{\varepsilon}$. 
(2) Up to passing to a subsequence, there exist $1 \leqslant \widetilde{N}_{0} \leqslant N$ and $(\mathbf{p}, \mathbf{D}) \in\left(\bar{\Omega}^{\tilde{N}_{0}}\right)^{*} \times \mathbb{Z}^{\tilde{N}_{0}}$ s.t. $(\mathbf{p}, \mathbf{D})$ is the cluster of $\left((\mathbf{a}, \mathbf{d})_{\varepsilon}\right)_{\varepsilon}$.

(3) If $(\mathbf{p}, \mathbf{D})$ is the cluster of $\left((\mathbf{a}, \mathbf{d})_{\varepsilon}\right)_{\varepsilon}$ then, denoting $\chi:=$ $\max _{k} \max _{i \in S_{k}}\left|a_{i}^{\varepsilon}-p_{k}\right|$, we have

$$
\left|\sum_{k=1}^{\tilde{N}_{0}} \sum_{i \in S_{k}}\right| d_{i}|| \xi_{0}\left(a_{i}^{\varepsilon}\right)-\xi_{0}\left(p_{k}\right)|| \leqslant C \chi
$$

and

$$
\left|\widetilde{V}\left[\zeta_{(\mathbf{a}, \mathbf{d})_{\varepsilon}}\right]-\widetilde{V}\left[\zeta_{(\mathbf{p}, \mathbf{D})}\right]\right| \leqslant C \sqrt{\chi}
$$

where $C$ depends only on $N, \sum\left|d_{i}\right|$ and $\Omega$.

Proof. - The two first assertions are obvious. Estimate (5.26) is direct by noting that $\xi_{0}$ a Lipschitzian function in $\Omega$. Estimate (5.27) is a direct consequence of Proposition 5.13.

We then have:

Corollary 5.20. - Assume that $\lambda, \delta, h_{\mathrm{ex}}$ satisfy (1.2) and (1.3) for some $K \geqslant 0$ independent of $\varepsilon$. Assume also $\delta^{2}|\ln \varepsilon| \leqslant 1$.

Let $\left\{\left(v_{\varepsilon}, A_{\varepsilon}\right) \mid 0<\varepsilon<1\right\} \subset \mathscr{H}$ be a family s.t. $\mathcal{F}\left(v_{\varepsilon}, A_{\varepsilon}\right) \leqslant \inf _{\mathscr{H}} \mathcal{F}+$ $K \ln |\ln \varepsilon|$ which is in the Coulomb gauge and let $\left\{\left(\mathbf{a}_{\varepsilon}, \mathbf{D}_{\varepsilon}\right)=(\mathbf{a}, \mathbf{d}) \mid 0<\varepsilon<1\right\}$ be a family of pseudo vortices associated to $\left\{\left(v_{\varepsilon}, A_{\varepsilon}\right) \mid 0<\varepsilon<1\right\}$ (indexed on $\mathcal{J}=\mathcal{J}_{\varepsilon}$ possibly empty).

(1) Letting $A_{v_{\varepsilon}} \in H^{1}\left(\Omega, \mathbb{R}^{2}\right)$ be defined by Lemma 5.8 we have

$$
\begin{aligned}
\mathcal{F}\left(v_{\varepsilon}, A_{\varepsilon}\right) & \geqslant \mathcal{F}\left(v_{\varepsilon}, A_{v_{\varepsilon}}\right) \\
& \geqslant h_{\mathrm{ex}}^{2} \mathbf{J}_{\mathbf{0}}+2 \pi h_{\mathrm{ex}} \sum_{i \in \mathcal{J}} d_{i} \xi_{0}\left(a_{i}\right)+F\left(v_{\varepsilon}\right)+\widetilde{V}\left[\zeta_{(\mathbf{a}, \mathbf{d})}\right]+o(1) .
\end{aligned}
$$

And then

$$
\mathcal{F}\left(v_{\varepsilon}, A_{\varepsilon}\right) \geqslant h_{\mathrm{ex}}^{2} \mathbf{J}_{\mathbf{0}}+2 \pi h_{\mathrm{ex}} \sum_{i \in \mathcal{J}} d_{i} \xi_{0}\left(a_{i}\right)+F\left(v_{\varepsilon}\right)+\mathcal{O}(1) .
$$

(2) Assume furthermore that $(\mathbf{a}, \mathbf{d})$ admits a cluster structure on $(\mathbf{p}, \mathbf{D})$. Then we have

$$
\mathcal{F}\left(v_{\varepsilon}, A_{\varepsilon}\right) \geqslant h_{\mathrm{ex}}^{2} \mathbf{J}_{\mathbf{0}}+2 \pi h_{\mathrm{ex}} \sum_{i \in \mathcal{J}} d_{i} \xi_{0}\left(a_{i}\right)+F\left(v_{\varepsilon}\right)+\widetilde{V}\left[\zeta_{(\mathbf{p}, \mathbf{D})}\right]+o(1) .
$$

Proof. - The lower bounds (5.28) and (5.29) are direct consequences of Theorem 5.6, Lemma 5.8, Remark 5.9 and Propositions 5.1, 5.7 and 5.12.

Estimate (5.30) is a direct consequence of Proposition 5.19 and (5.28).

We then have the following corollary. 
Corollary 5.21. - Assume that $\lambda, \delta, h_{\mathrm{ex}}$ satisfy (1.2) and (1.3). Assume also $\delta^{2}|\ln \varepsilon| \leqslant 1$.

Let $\left(v_{\varepsilon}\right)_{0<\varepsilon<1} \subset H^{1}(\Omega, \mathbb{C})$ be s.t. $\left|v_{\varepsilon}\right| \ngtr 1 / 2$ in $\Omega$ and assume the existence of $\left(B_{\varepsilon}\right)_{0<\varepsilon<1} \subset H^{1}\left(\Omega, \mathbb{R}^{2}\right)$ s.t. $\left(v_{\varepsilon}, B_{\varepsilon}\right)$ is in the Coulomb gauge and $\mathcal{F}\left(v_{\varepsilon}, B_{\varepsilon}\right) \leqslant \inf _{\mathscr{H}} \mathcal{F}+\mathcal{O}(\ln |\ln \varepsilon|)$. Assume also that $\left(\mathbf{a}_{\varepsilon}, \mathbf{D}_{\varepsilon}\right)=(\mathbf{a}, \mathbf{D})$ are pseudo-vortices as in Definition 5.11 for $v_{\varepsilon}$ (note that we thus have $\left.\sum\left|d_{i}\right|=\mathcal{O}(1)\right)$, then

$$
\mathcal{F}\left(v_{\varepsilon}, A_{(\mathbf{a}, \mathbf{D})}\right)=h_{\mathrm{ex}}^{2} \mathbf{J}_{\mathbf{0}}+2 \pi h_{\mathrm{ex}} \sum d_{i} \xi_{0}\left(a_{i}\right)+F\left(v_{\varepsilon}\right)+\widetilde{V}\left[\zeta_{(\mathbf{a}, \mathbf{D})}\right]+o(1) .
$$

where $A_{(\mathbf{a}, \mathbf{D})}:=h_{\mathrm{ex}} \nabla^{\perp} \xi_{0}+\nabla^{\perp} \zeta_{(\mathbf{a}, \mathbf{D})}$.

Consequently we get

$$
\begin{aligned}
F\left(v_{\varepsilon}\right) & \leqslant 2 \pi h_{\text {ex }} \sum d_{i}\left|\xi_{0}\left(a_{i}\right)\right|+\mathcal{O}(\ln |\ln \varepsilon|) \\
& \leqslant \pi b^{2} \sum\left|d_{i}\right||\ln \varepsilon|+\mathcal{O}(\ln |\ln \varepsilon|) .
\end{aligned}
$$

Proof. - Corollary 5.21 is a direct consequence of $\inf \mathscr{H} \mathcal{F} \leqslant h_{\mathrm{ex}}^{2} \mathbf{J}_{\mathbf{0}}$, Corollary 5.20 and Propositions 5.7 and 5.13.

Remark 5.22. - We may state an analog of Corollary 5.21 if $(\mathbf{a}, \mathbf{d})$ admits a structure of cluster.

\section{Renormalized energies}

\subsection{Macroscopic renormalized energy (at scale 1)}

We consider in this section:

- $N \in \mathbb{N}^{*}, \mathbf{z}=\mathbf{z}^{(n)} \in\left(\Omega^{N}\right)^{*}:=\left\{\left(z_{1}, \ldots, z_{N}\right) \subset \Omega \mid z_{i} \neq z_{j}\right.$ for $\left.i \neq j\right\}$,

- $\mathbf{D}=\left(d_{1}, \ldots, d_{N}\right) \in \mathbb{Z}^{N}$.

- $\hbar=\hbar(\mathbf{z}):=\min _{i} \operatorname{dist}\left(z_{i}, \partial \Omega\right)$

We are going to deal with functions defined in the set $\Omega$ perforated by disks with radius $\widetilde{r}=\widetilde{r}_{n} \downarrow 0$ :

$$
\Omega_{\tilde{r}}=\Omega_{\tilde{r}}(\mathbf{z}):=\Omega \backslash \bigcup_{i} \overline{B\left(z_{i}, \widetilde{r}\right)} .
$$

We assume

$$
\widetilde{r}<\frac{1}{8} \min \left\{\min _{i \neq j}\left|z_{i}-z_{j}\right| ; \hbar\right\} .
$$


For a radius $\widetilde{r}>0$ s.t. (6.1) is satisfied, we consider the set of functions

$$
\mathcal{I}_{\tilde{r}}^{\mathrm{deg}}:=\left\{w \in H^{1}\left(\Omega_{\tilde{r}}, \mathbb{S}^{1}\right) \mid \operatorname{deg}_{\partial B\left(z_{i}, \tilde{r}\right)}(w)=d_{i} \text { for } i \in\{1, \ldots, N\}\right\}
$$

and

$$
\mathcal{I}_{\tilde{r}}^{\text {Dir }}:=\left\{w \in H^{1}\left(\Omega_{\tilde{r}}, \mathbb{S}^{1}\right) \mid \begin{array}{c}
w\left(z_{i}+\widetilde{r} \mathrm{e}^{\imath \theta}\right)=C_{i} \mathrm{e}^{\imath d_{i} \theta} \text { for } i \in\{1, \ldots, N\}, \\
\left(C_{1}, \ldots, C_{N}\right) \in\left(\mathbb{S}^{1}\right)^{N}
\end{array}\right\} .
$$

In this section we are interested in the minimization of the Dirichlet functional in $\mathcal{I}_{\tilde{r}}^{\text {deg }}$ and $\mathcal{I}_{\tilde{r}}^{\text {Dir }}$.

Before beginning we state an easy result proved by direct minimization (the proof is left to the reader, see [4]).

Proposition 6.1. - For $N \geqslant 1,(\mathbf{z}, \mathbf{d}) \in\left(\Omega^{N}\right)^{*} \times \mathbb{Z}^{N}$ and $\widetilde{r}>0$ s.t. (6.1) is satisfied, the following minimization problems admit solutions:

$$
I_{\tilde{r}}^{\mathrm{deg}}=I_{\tilde{r}}^{\operatorname{deg}}(\mathbf{z}, \mathbf{d}):=\inf _{w \in \mathcal{I}_{\tilde{r}}^{\operatorname{deg}}} \frac{1}{2} \int_{\Omega_{\tilde{r}}}|\nabla w|^{2}
$$

and

$$
I_{\tilde{r}}^{\text {Dir }}=I_{\tilde{r}}^{\text {Dir }}(\mathbf{z}, \mathbf{d}):=\inf _{w \in \mathcal{I}_{\tilde{r}}^{\text {Dir }}} \frac{1}{2} \int_{\Omega_{\tilde{r}}}|\nabla w|^{2} .
$$

Moreover, these solutions are unique up to the multiplication by an $\mathbb{S}^{1}$ constant.

\subsubsection{Study of $I_{\tilde{r}}^{\mathrm{deg}}$ and $I_{\tilde{r}}^{\mathrm{Dir}}$}

Following [4], it is standard to define the canonical harmonic map associated to $(\mathbf{z}, \mathbf{d})$.

Definition 6.2. - Let $N \in \mathbb{N}^{*}$ and $(\mathbf{z}, \mathbf{d}) \in\left(\Omega^{N}\right)^{*} \times \mathbb{Z}^{N}$.

A function $w_{\star}^{(\mathbf{z}, \mathbf{d})} \in \cap_{0<p<2} W^{1, p}\left(\Omega, \mathbb{S}^{1}\right) \cap C^{\infty}\left(\Omega \backslash\left\{z_{1}, \ldots, z_{N}\right\}, \mathbb{S}^{1}\right)$ is the canonical harmonic map associated to the singularities $(\mathbf{z}, \mathbf{d})$ if

$$
w_{\star}^{(\mathbf{z}, \mathbf{d})}(z)=\mathrm{e}^{\imath \varphi_{\star}(z)} \prod_{i=1}^{N}\left(\frac{z-z_{i}}{\left|z-z_{i}\right|}\right)^{d_{i}} \text { with }\left\{\begin{array}{l}
\varphi_{\star} \text { is harmonic in } \Omega, \\
\partial_{\nu} w_{\star}^{(\mathbf{z}, \mathbf{d})}=0 \text { on } \partial \Omega, \\
\int_{\partial \Omega} \varphi_{\star}=0 .
\end{array}\right.
$$

Remark 6.3. - In this framework, it is classic to define $\Phi_{\star}^{(\mathbf{z}, \mathbf{d})}$ (with the notation of Definition 6.2), the unique solution of

$$
\begin{cases}\Delta \Phi_{\star}^{(\mathbf{z}, \mathbf{d})}=2 \pi \sum_{i=1}^{N} d_{i} \delta_{z_{i}} & \text { in } \Omega \\ \Phi_{\star}^{(\mathbf{z}, \mathbf{d})}=0 & \text { on } \partial \Omega\end{cases}
$$


This function satisfies $\nabla^{\perp} \Phi_{\star}^{(\mathbf{z}, \mathbf{d})}=w_{\star}^{(\mathbf{z}, \mathbf{d})} \wedge \nabla w_{\star}^{(\mathbf{z}, \mathbf{d})}$. Moreover, by denoting $R_{(\mathbf{z}, \mathbf{d})}$ the unique solution of

$$
\begin{cases}\Delta R_{(\mathbf{z}, \mathbf{d})}=0 & \text { in } \Omega \\ R_{(\mathbf{z}, \mathbf{d})}(z)=-\sum_{i} d_{i} \ln \left|z-z_{i}\right| & \text { on } \partial \Omega\end{cases}
$$

we have $\Phi_{\star}^{(\mathbf{z}, \mathbf{d})}(z)=\sum_{i} d_{i} \ln \left|z-z_{i}\right|+R_{(\mathbf{z}, \mathbf{d})}(z)$.

We first study the asymptotic behavior of minimizers of $I_{\tilde{r}}^{\mathrm{deg}}(\mathbf{z}, \mathbf{d})$ when $\widetilde{r} \rightarrow 0$.

Proposition 6.4. - Let $N \in \mathbb{N}^{*}$ and $(\mathbf{z}, \mathbf{d})=(\mathbf{z}, \mathbf{d})^{(n)} \subset\left(\Omega^{N}\right)^{*} \times \mathbb{Z}^{N}$. We write $\hbar=\hbar(\mathbf{z})$ and we assume that $\sum_{i}\left|d_{i}\right|=\mathcal{O}(1)$.

For $\tilde{r}>0$ s.t. (6.1) is satisfied, we may consider $w_{\tilde{r}}^{(\mathbf{z}, \mathbf{d})}$, the unique solution of the problem

$$
I_{\tilde{r}}^{\operatorname{deg}}(\mathbf{z}, \mathbf{d}):=\inf _{w \in \mathcal{I}_{\tilde{r}}^{\operatorname{deg}}} \frac{1}{2} \int_{\Omega_{\tilde{r}}}|\nabla w|^{2}
$$

of the form

$$
w_{\tilde{r}}^{(\mathbf{z}, \mathbf{d})}(z)=\mathrm{e}^{\imath \varphi_{\tilde{r}}(z)} \prod_{i=1}^{N}\left(\frac{z-z_{i}}{\left|z-z_{i}\right|}\right)^{d_{i}} \text { with }\left\{\begin{array}{l}
\varphi_{\tilde{r}} \in H^{1} \cap C^{\infty}\left(\Omega_{\tilde{r}}, \mathbb{R}\right) \\
\int_{\partial \Omega} \varphi_{\tilde{r}}=0 .
\end{array}\right.
$$

We thus have the existence of $C>0$ (depending only on $\Omega, N$ and the bound of $\left.\sum_{i}\left|d_{i}\right|\right)$ s.t.

$$
\left\|\nabla w_{\star}^{(\mathbf{z}, \mathbf{d})}\right\|_{L^{\infty}\left(\Omega_{\tilde{r}}\right)} \leqslant \frac{C(1+|\ln \widetilde{r}|)}{\widetilde{r}}
$$

We denote

$$
X:= \begin{cases}\frac{\tilde{r}(1+|\ln (\hbar)|)}{\hbar}\left(1+\frac{\tilde{r}(1+|\ln (\hbar)|)}{\hbar}\right) & \text { if } N=1 \\ \left(\frac{\tilde{r}}{\min _{i \neq j}\left|z_{i}-z_{j}\right|}+\frac{\tilde{r}(1+|\ln (\hbar)|)}{\hbar}\right)\left(1+\frac{\tilde{r}(1+|\ln (\hbar)|)}{\hbar}\right) & \text { if } N \geqslant 2\end{cases}
$$

and we have

$$
\begin{gathered}
\left\|\varphi_{\tilde{r}}-\varphi_{\star}\right\|_{H^{1}\left(\Omega_{\tilde{r}}\right)}^{2} \leqslant C X \\
0 \leqslant \frac{1}{2} \int_{\Omega_{\tilde{r}}}\left|\nabla w_{\star}^{(\mathbf{z}, \mathbf{d})}\right|^{2}-\inf _{w \in \mathcal{I}_{\tilde{r}}^{\operatorname{deg}}} \frac{1}{2} \int_{\Omega_{\tilde{r}}}|\nabla w|^{2} \leqslant C X .
\end{gathered}
$$

Moreover, if there exists $\eta>0$ (independent of $n$ ) s.t. $\hbar>\eta$ then (6.7) may be refined into

$$
\left\|\nabla w_{\star}^{(\mathbf{z}, \mathbf{d})}\right\|_{L^{\infty}\left(\Omega_{\tilde{r}}\right)} \leqslant \frac{C}{\widetilde{r}} .
$$

The proof of Proposition 6.4 is in Appendix D.1.

By adapting the proof of Proposition 5.1 in [17] we have 
Proposition 6.5. - For $N \geqslant 1$, there exists an application $W_{N}^{\text {macro }}=$ $W^{\text {macro }}:\left(\Omega^{N}\right)^{*} \times \mathbb{Z}^{N} \rightarrow \mathbb{R}$ s.t. for sequences $(\mathbf{z}, \mathbf{d})=(\mathbf{z}, \mathbf{d})^{(n)} \in\left(\Omega^{N}\right)^{*} \times \mathbb{Z}^{N}$ and $\widetilde{r}=\widetilde{r}_{n} \rightarrow 0$ satisfying (6.1) and s.t. $\mathbf{D}$ is independent of $n$, there exists $C \geqslant 1$ (depending only on $N, \sum\left|d_{i}\right|$ and $\Omega$ ) s.t.

$$
\left.\left|\frac{1}{2} \int_{\Omega_{\tilde{r}}}\right| \nabla w_{\star}^{(\mathbf{z}, \mathbf{d})}\right|^{2}-\pi \sum_{i} d_{i}^{2}|\ln \widetilde{r}|-W^{\text {macro }}(\mathbf{z}, \mathbf{d}) \mid \leqslant C X
$$

with

$$
\begin{aligned}
& W^{\text {macro }}(\mathbf{z}, \mathbf{d})=-\pi \sum_{i \neq j} d_{i} d_{j} \ln \left|z_{i}-z_{j}\right|-\pi \sum_{i} d_{i} R_{(\mathbf{z}, \mathbf{d})}\left(z_{i}\right), \\
& R_{(\mathbf{z}, \mathbf{d})} \in C^{\infty}(\Omega, \mathbb{R}) \text { satisfies }\left\|R_{(\mathbf{z}, \mathbf{d})}\right\|_{L^{\infty}(\Omega)} \leqslant C(1+|\ln \hbar|) .
\end{aligned}
$$

Proposition 6.5 is proved in D.2. We immediately obtain from Proposition 6.5 the following corollary.

Corollary 6.6. - Under the hypotheses of Proposition 6.5 and assuming that there exists $C_{1}>0$ (independent of $r$ ) s.t. $\frac{\tilde{r}(1+|\ln \hbar|)}{\hbar} \leqslant C_{1}$, there is $C>1$ (depending only on $\Omega, N, \sum_{i}\left|d_{i}\right|$ and $C_{1}$ ) s.t. $\int_{\Omega_{\tilde{r}}}\left|\nabla w_{\star}^{(\mathbf{z}, \mathbf{d})}\right|^{2} \leqslant$ $C|\ln \widetilde{r}|$.

We end this section by linking $I_{\tilde{r}}^{\mathrm{deg}}$ and $I_{\tilde{r}}^{\mathrm{Dir}}$.

Proposition 6.7. - Let $N \geqslant 1, \mathbf{z} \in\left(\Omega^{N}\right)^{*}$ and $\widetilde{r}=\widetilde{r}_{n} \downarrow 0$ satisfying (6.1). Assume $\frac{\tilde{r}}{\hbar} \rightarrow 0$ and if $N \geqslant 2$, we also assume $\frac{\tilde{r}}{\min _{i \neq j}\left|z_{i}-z_{j}\right|} \rightarrow 0$.

Let

$$
\eta:= \begin{cases}10^{-1} \hbar & \text { if } N=1 \\ 10^{-1} \min \left\{\hbar ; \min _{i \neq j}\left|z_{i}-z_{j}\right|\right\} & \text { if } N \geqslant 2 .\end{cases}
$$

Assume furthermore

$$
Z:=\frac{1}{\ln (\eta / \widetilde{r})}\left[\frac{\eta(1+|\ln (\hbar)|)}{\hbar}+1\right]^{2} \rightarrow 0 .
$$

Then for $\mathbf{D} \in \mathbb{Z}^{N}$ (independent of $n$ ), there exists $C>1$ (depending only on $\Omega, N$ and $\left.\sum\left|d_{i}\right|\right)$ s.t.

$$
0 \leqslant \inf _{w \in \mathcal{I}_{\tilde{r}}^{\text {Dir }}} \frac{1}{2} \int_{\Omega_{\tilde{r}}}|\nabla w|^{2}-\inf _{w \in \mathcal{I}_{\tilde{r}}^{\operatorname{deg}}} \frac{1}{2} \int_{\Omega_{\tilde{r}}}|\nabla w|^{2} \leqslant C(X+Z) .
$$

Proposition 6.7 is proved Appendix D.3. 


\subsubsection{Macroscopic renormalized energy and cluster of vortices}

We first state an easy lemma.

LEMMA 6.8.

(1) Let $N \in \mathbb{N}^{*}$ and $\mathbf{D} \in \mathbb{Z}^{N}$. Let $\chi>0$ and $\mathbf{z}, \mathbf{z}^{\prime} \in\left(\Omega^{N}\right)^{*}$ be s.t. for $i \in\{1, \ldots, N\}$ we have $\left|z_{i}-z_{i}^{\prime}\right| \leqslant \chi$. Then we have

$$
\left\|R_{(\mathbf{z}, \mathbf{d})}-R_{\left(\mathbf{z}^{\prime}, \mathbf{D}\right)}\right\|_{L^{\infty}(\Omega)} \leqslant \sum_{i}\left|d_{i}\right| \frac{\chi}{\max \left\{\hbar(\mathbf{z}), \hbar\left(\mathbf{z}^{\prime}\right)\right\}} .
$$

(2) Let $1 \leqslant \widetilde{N}_{0} \leqslant N, \mathbf{p} \in\left(\Omega^{\tilde{N}_{0}}\right)^{*},(\mathbf{z}, \mathbf{d})=(\mathbf{z}, \mathbf{d})^{(n)} \in\left(\Omega^{N}\right)^{*} \times \mathbb{Z}^{N}$ be s.t. $\mathbf{D}$ is independent of $n$ and for $i \in\{1, \ldots, N\}$ there exists $k \in$ $\left\{1, \ldots, \widetilde{N}_{0}\right\}$ s.t. $z_{i} \rightarrow p_{k}$. We let $\chi:=\max _{i} \operatorname{dist}\left(z_{i},\left\{p_{1}, \ldots, p_{\tilde{N}_{0}}\right\}\right)$. For $k \in\left\{1, \ldots, \widetilde{N}_{0}\right\}$ we let $D_{k}:=\sum_{z_{i} \rightarrow p_{k}} d_{i}$ and $\mathbf{D}=\left(D_{1}, \ldots, D_{\tilde{N}_{0}}\right)$. Then we have

$$
\left\|R_{(\mathbf{z}, \mathbf{d})}-R_{(\mathbf{p}, \mathbf{D})}\right\|_{L^{\infty}(\Omega)} \leqslant \sum_{i}\left|d_{i}\right| \frac{\chi}{\hbar(\mathbf{p})} .
$$

Proof. - The first assertion is obtained with the help of the maximum principle and the bound $\left|R_{(\mathbf{z}, \mathbf{d})}-R_{\left(\mathbf{z}^{\prime}, \mathbf{D}\right)}\right| \leqslant \sum_{i}\left|d_{i}\right| \frac{\chi}{\max \left\{\hbar(\mathbf{z}), \hbar\left(\mathbf{z}^{\prime}\right)\right\}}$ on $\partial \Omega$. The second assertion follows by the same way.

With Lemma 6.8 we may exploit a structure of cluster for $W^{\text {macro }}$.

Proposition 6.9. - Let $1 \leqslant \widetilde{N}_{0} \leqslant N, \mathbf{p} \in\left(\Omega^{\tilde{N}_{0}}\right)^{*}$ (independent of $n$ ) and write

$$
\gamma_{\mathbf{p}}:= \begin{cases}1 & \text { if } \widetilde{N}_{0}=1, \\ \min _{k \neq l}\left|p_{k}-p_{l}\right| & \text { otherwise. }\end{cases}
$$

Let $(\mathbf{z}, \mathbf{d})=(\mathbf{z}, \mathbf{d})^{(n)} \in\left(\Omega^{N}\right)^{*} \times \mathbb{Z}^{N}$ be s.t. $\mathbf{D}$ is independent of $n$ and for $i \in\{1, \ldots, N\}$ there exists $k \in\left\{1, \ldots, \widetilde{N}_{0}\right\}$ s.t. $z_{i} \rightarrow p_{k}$. We denote $\chi:=\max _{i} \operatorname{dist}\left(z_{i},\left\{p_{1}, \ldots, p_{\tilde{N}_{0}}\right\}\right)$.

For $k \in\left\{1, \ldots, \widetilde{N}_{0}\right\}$ we denote $D_{k}:=\sum_{z_{i} \rightarrow p_{k}} d_{i}$ and $\mathbf{D}=\left(D_{1}, \ldots, D_{\tilde{N}_{0}}\right)$. Then there exists $C \geqslant 1$ (depending only on $\Omega, N$ and $\sum\left|d_{i}\right|$ ) s.t.

$$
\begin{array}{r}
\left|W_{N}^{\text {macro }}(\mathbf{z}, \mathbf{d})-\left(W_{\tilde{N}_{0}}^{\operatorname{macro}}(\mathbf{p}, \mathbf{D})-\pi \sum_{k=1}^{\tilde{N}_{0}} \sum_{\substack{z_{i}, z_{j} \rightarrow p_{k} \\
i \neq j}} d_{i} d_{j} \ln \left|z_{i}-z_{j}\right|\right)\right| \\
\leqslant C \chi\left(\frac{1+|\ln [\hbar(\mathbf{p})]|}{\hbar(\mathbf{p})}+\frac{1}{\gamma_{\mathbf{p}}}\right) .
\end{array}
$$


Proof. - We have

$$
\begin{aligned}
& W^{\text {macro }}(\mathbf{z}, \mathbf{d})=-\pi \sum_{k=1}^{\tilde{N}_{0}} \sum_{\substack{z_{i}, z_{j} \rightarrow p_{k} \\
i \neq j}} d_{i} d_{j} \ln \left|z_{i}-z_{j}\right| \\
& -\pi \sum_{\substack{z_{i} \rightarrow p_{k} \\
z_{j} \rightarrow p_{l} \\
k \neq l}} d_{i} d_{j} \ln \left|z_{i}-z_{j}\right|-\pi \sum_{i} d_{i} R_{(\mathbf{z}, \mathbf{d})}\left(z_{i}\right) .
\end{aligned}
$$

It is easy to check that

$$
\sum_{\substack{z_{i} \rightarrow p_{k} \\ z_{j} \rightarrow p_{l} \\ k \neq l}} d_{i} d_{j} \ln \left|z_{i}-z_{j}\right|=\sum_{k \neq l} D_{k} D_{l} \ln \left|p_{k}-p_{l}\right|+H
$$

with $H \leqslant 4\left(\sum_{i}\left|d_{i}\right|\right)^{2} \frac{\chi}{\gamma_{\mathbf{p}}}$ for sufficiently large $n$.

On the other hand, from Lemma 6.8 (second assertion), we have

$$
\left\|R_{(\mathbf{z}, \mathbf{d})}-R_{(\mathbf{p}, \mathbf{D})}\right\|_{L^{\infty}(\Omega)} \leqslant \sum_{i}\left|d_{i}\right| \frac{\chi}{\max \{\hbar(\mathbf{z}), \hbar(\mathbf{p})\}} .
$$

From standard pointwise estimates for the gradient of harmonic functions (see (D.6)) there exists $C \geqslant 1$ depending only on $\Omega, \sum\left|D_{k}\right|$ and $N$ (here we used $\left.1 \leqslant \widetilde{N}_{0} \leqslant N\right)$ s.t. for $z_{i} \rightarrow p_{k}$ we have $\left|R_{(\mathbf{p}, \mathbf{D})}\left(z_{i}\right)-R_{(\mathbf{p}, \mathbf{D})}\left(p_{k}\right)\right| \leqslant$ $C \chi \frac{1+|\ln [\hbar(\mathbf{p})]|}{\hbar(\mathbf{p})}$.

Then, up to changing the value of $C$, we have

$$
\left|\sum_{i} d_{i} R_{(\mathbf{z}, \mathbf{d})}\left(z_{i}\right)-\sum_{k} D_{k} R_{(\mathbf{p}, \mathbf{D})}\left(p_{k}\right)\right| \leqslant C \chi \frac{1+|\ln [\hbar(\mathbf{p})]|}{\hbar(\mathbf{p})} .
$$

By combining (6.12) and (6.13) we get the result.

\subsection{Mesoscopic renormalized energy (at scale $h_{\mathrm{ex}}^{-1 / 2}$ )}

From the work of Sandier and Serfaty we may obtain mesoscopic informations. To this end we need to assume a non degeneracy assumption for minimal points of $\xi_{0}$. So we assume in this section that Hypothesis (1.5) holds.

Let

$$
\eta_{\Omega}:= \begin{cases}10^{-3} \min \{1 ; \operatorname{dist}(\Lambda, \partial \Omega)\} & \text { if } N_{0}=1 \\ 10^{-3} \min \left\{1 ; \operatorname{dist}(\Lambda, \partial \Omega) ; \min _{k \neq l}\left|p_{k}-p_{l}\right|\right\} & \text { if } N_{0} \geqslant 2 .\end{cases}
$$


For $p \in \Lambda$, by applying Lemma 11.1 in [16] in the disk $B\left(p, \eta_{\Omega}\right)$, we get the following proposition.

Proposition 6.10. - Assume that Hypothesis (1.5) holds. Let $D \in \mathbb{N}^{*}$ and $h_{\mathrm{ex}} \uparrow \infty$ when $\varepsilon \rightarrow 0$. Then for $p \in \Lambda$ and $R=R(\varepsilon) \rightarrow 0$ s.t. $R \sqrt{h_{\mathrm{ex}}} \rightarrow$ $\infty$ we have

$$
\begin{array}{r}
\inf _{\mathbf{z} \in\left[B(p, R)^{D}\right]^{*}}\left\{-\pi \sum_{i \neq j} \ln \left|z_{i}-z_{j}\right|+2 \pi h_{\mathrm{ex}} \sum_{i}\left[\xi_{0}\left(z_{i}\right)-\xi_{0}(p)\right]\right\} \\
=\frac{\pi}{2}\left(D^{2}-D\right) \ln \left(\frac{h_{\mathrm{ex}}}{D}\right)+C_{p, D}+o(1)
\end{array}
$$

with

$$
C_{p, D}:=\min _{\left[\left(\mathbb{R}^{2}\right)^{D}\right]^{*}} W_{p, D}^{\text {meso }}
$$

and

$$
\begin{array}{cc}
W_{p, D}^{\text {meso }}: & {\left[\left(\mathbb{R}^{2}\right)^{D}\right]^{*} \longrightarrow \mathbb{R}} \\
\mathbf{x}=\left(x_{1}, \ldots, x_{D}\right) & \longmapsto-\pi \sum_{i \neq j} \ln \left|x_{i}-x_{j}\right|+\pi D \sum_{i=1}^{D} Q_{p}\left(x_{i}\right) .
\end{array}
$$

where $Q_{p}(x):=x \cdot \operatorname{Hess}_{\xi_{0}}(p) x, \operatorname{Hess}_{\xi_{0}}(p)$ is the Hessian matrix of $\xi_{0}$ at $p$.

Moreover the infimum in (6.15) is reached and if $\mathbf{z}^{\varepsilon} \in\left[B(p, R)^{D}\right]^{*}$ is s.t.

$$
\begin{aligned}
-\pi \sum_{i \neq j} \ln \left|z_{i}^{\varepsilon}-z_{j}^{\varepsilon}\right|+2 \pi h_{\mathrm{ex}} \sum_{i}\left[\xi_{0}\left(z_{i}^{\varepsilon}\right)-\xi_{0}(p)\right] \\
=\frac{\pi}{2}\left(D^{2}-D\right) \ln \left(\frac{h_{\mathrm{ex}}}{D}\right)+C_{p, D}+o(1)
\end{aligned}
$$

then for all sequence $\varepsilon=\varepsilon_{n} \downarrow 0$, up to a subsequence, denoting $\ell=\sqrt{\frac{D}{h_{\mathrm{ex}}}}$ and $\breve{z}_{i}^{\varepsilon}=\frac{z_{i}^{\varepsilon}-p}{\ell}$, we have $\breve{\mathbf{z}}^{\varepsilon}=\left(\breve{z}_{1}^{\varepsilon}, \ldots, \breve{z}_{D}^{\varepsilon}\right)$ which converges to a minimizer of $W_{p, D}^{\text {meso }}$. In particular $\left|\breve{z}_{i}^{\varepsilon}\right| \leqslant C_{\Omega, D}$ with $C_{\Omega, D}>0$ which depends only on $\Omega$ and $D$.

\subsection{Microscopic renormalized energy (at scale $\lambda \delta$ )}

The location of the vorticity defects at scale $\lambda \delta$ (inside a connected component of $\omega_{\varepsilon}$ ) is given by the microscopic renormalized energy exactly as in the case without magnetic field. In order to define the microscopic renormalized energy we need some notation. Recall that the pinning term $a_{\varepsilon}: \Omega \rightarrow\{b, 1\}$ is obtained (see Section 2.3) from a smooth bounded simply connected set $\omega$ s.t. $0 \in \omega \subset \bar{\omega} \subset Y:=(-1 / 2,1 / 2)^{2}$. The construction of the 
pinning term uses two parameters $\delta=\delta(\varepsilon)$ (the parameter of period) and $\lambda=\lambda(\varepsilon)$ (the parameter of dilution). For $x_{0} \in \omega$ and a sequence $\varepsilon=\varepsilon_{n} \downarrow 0$, we consider $\widehat{x}_{\varepsilon} \in \omega$ s.t. $\widehat{x}_{\varepsilon} \rightarrow x_{0} \in \omega$.

Let $m_{\varepsilon} \in \mathbb{Z}^{2}$ be s.t. the cell $Y_{\varepsilon}=\delta\left(m_{\varepsilon}+Y\right)$ satisfies $\bar{Y}_{\varepsilon} \subset \Omega$. We then denote $z_{\varepsilon}=\delta\left[m_{\varepsilon}+\lambda \widehat{x}_{\varepsilon}\right]$. It is proved in [7, Estimates (9) and (10)] that for $R=R_{\varepsilon} \gg \lambda \delta$ and $r=r_{\varepsilon} \ll \lambda \delta$, denoting $\widehat{R}=R /(\lambda \delta), \widehat{r}=r /(\lambda \delta), \mathcal{D}_{\varepsilon}=$ $B\left(\delta m_{\varepsilon}, R\right) \backslash \overline{B\left(z_{\varepsilon}, r\right)}, \widehat{\mathcal{D}}_{\varepsilon}=B(0, \widehat{R}) \backslash \overline{B\left(\widehat{x}_{\varepsilon}, \widehat{r}\right)}$ and $\widehat{\mathcal{D}}=B(0, \widehat{R}) \backslash \overline{B\left(x_{0}, \widehat{r}\right)}$ :

$$
\begin{aligned}
\inf _{\substack{w \in H^{1}\left(\mathcal{D}_{\varepsilon}, \mathbb{S}^{1}\right) \\
\operatorname{deg}(w)=1}} \frac{1}{2} \int_{\mathcal{D}_{\varepsilon}} U_{\varepsilon}^{2}|\nabla w|^{2}= & \inf _{\begin{array}{c}
w \in H^{1}\left(\mathcal{D}_{\varepsilon}, \mathbb{S}^{1}\right) \\
w\left(z_{\varepsilon}+R \mathrm{e}^{\theta \theta}\right)=\mathrm{e}^{\theta \theta} \\
w\left(x_{\varepsilon}+r \mathrm{e}^{2 \theta}\right)=\operatorname{Cst~}^{2 \theta}
\end{array}} \frac{1}{2} \int_{\mathcal{D}_{\varepsilon}} U_{\varepsilon}^{2}|\nabla w|^{2}+o_{\varepsilon}(1) \\
& =\inf _{\substack{\hat{w} \in H^{1}\left(\hat{\mathcal{D}}_{\varepsilon}, \mathbb{S}^{1}\right) \\
\operatorname{deg}(w)=1}} \frac{1}{2} \int_{\hat{\mathcal{D}}_{\varepsilon}} a^{2}|\nabla \widehat{w}|^{2}+o_{\varepsilon}(1) .
\end{aligned}
$$

Moreover from the main result in [8], we have the existence of an application $\widetilde{W}^{\text {micro }}: \omega \rightarrow \mathbb{R}$ (depending only on $\omega$ and $b$ ) s.t.

$$
\inf _{\substack{\hat{w} \in H^{1}\left(\hat{\mathcal{D}}_{\varepsilon}, \mathbb{S}^{1}\right) \\ \operatorname{deg}(w)=1}} \frac{1}{2} \int_{\hat{\mathcal{D}}_{\varepsilon}} a^{2}|\nabla \widehat{w}|^{2}=f_{\omega}(\widehat{R})+b^{2} \pi|\ln (\widehat{r})|+\widetilde{W}^{\text {micro }}\left(x_{0}\right)+o(1) .
$$

where $f_{\omega}(\widehat{R}):=\inf _{w \in H^{1}\left[B(0, \hat{R}) \backslash \bar{\omega}, \mathbb{S}^{1}\right]} \frac{1}{2} \int_{B(0, \hat{R}) \backslash \bar{\omega}}|\nabla w|^{2}$.

It is clear that there exists $C_{\omega} \in \mathbb{R}$ (depending only on $\omega$ ) s.t. when $\widehat{R} \rightarrow \infty$ we have $f_{\omega}(\widehat{R})=\pi \ln (\widehat{R})+C_{\omega}+o(1)$.

Then, by denoting $W^{\text {micro }}\left(x_{0}\right):=\widetilde{W}^{\text {micro }}\left(x_{0}\right)+C_{\omega}$, we get from (6.20):

$$
\inf _{\substack{\hat{w} \in H^{1}\left(\hat{\mathcal{D}}, \mathbb{S}^{1}\right) \\ \operatorname{deg}(w)=1}} \frac{1}{2} \int_{\hat{\mathcal{D}}} a^{2}|\nabla \widehat{w}|^{2}=\pi \ln (\widehat{R})+b^{2} \pi|\ln (\widehat{r})|+W^{\text {micro }}\left(x_{0}\right)+o(1) .
$$

Moreover, from [9] we know that $W^{\text {micro }}$ admits minimizers in $\omega$.

\section{Sharp upper bound: construction of a test function}

From now on we assume that Hypothesis (1.5) holds. We thus may use for $p \in \Lambda$ and $D \in \mathbb{N}^{*}$ the constant $C_{p, D}$ defined in (6.16). We denote also $C_{p, 0}:=0$.

We let for $d \in \mathbb{N}^{*}$ :

$$
\Lambda_{d}:=\left\{\mathbf{D} \in\left\{\left\lceil\frac{d}{N_{0}}\right\rceil ;\left\lfloor\frac{d}{N_{0}}\right\rfloor\right\}^{N_{0}} \mid \sum_{k=1}^{N_{0}} D_{k}=d\right\},
$$




$$
\overline{\mathcal{W}}_{d, \Omega}=\overline{\mathcal{W}}_{d}:=\min _{\mathbf{D} \in \Lambda_{d}}\left\{W^{\text {macro }}(\mathbf{p}, \mathbf{D})+\sum_{k=1}^{N_{0}} C_{p_{k}, D_{k}}+\widetilde{V}\left[\zeta_{(\mathbf{p}, \mathbf{D})}\right]\right\}
$$

where, for $x \in \mathbb{R},\lceil x\rceil$ is the ceiling of $x,\lfloor x\rfloor$ is the floor of $x, W^{\text {macro }}(\cdot)$ is defined in Proposition 6.5 and $\widetilde{V}\left[\zeta_{(\mathbf{p}, \mathbf{D})}\right]$ is defined in Proposition 5.13.

We now state an easy lemma whose proof is left to the reader.

Lemma 7.1. - Let $d \in \mathbb{N}^{*}$ and $\mathbf{D} \in \Lambda_{d}$. Then the following quantities are independent of $\mathbf{D}$ :

$$
\begin{aligned}
& \mathscr{L}_{1}(d):=\frac{\pi}{2}\left[\left(\sum_{k=1}^{N_{0}} D_{k}^{2}\right)-d\right], \\
& \mathscr{L}_{2}(d):=\overline{\mathcal{W}}_{d}+\frac{\pi}{2} \sum_{\substack{k=1 \\
\text { s.t. } \bar{D}_{k} \geqslant 1}}^{N_{0}}\left(D_{k}-D_{k}^{2}\right) \ln \left(D_{k}\right) .
\end{aligned}
$$

Moreover: $d \leqslant N_{0} \Longleftrightarrow \mathscr{L}_{1}(d)=0 \Longleftrightarrow \mathscr{L}_{2}(d)=\overline{\mathcal{W}}_{d}$.

Notation 7.2. - We let $\mathscr{L}_{1}(0)=\mathscr{L}_{2}(0)=0$.

The main result of this section is the following proposition.

Proposition 7.3. - Assume that $h_{\mathrm{ex}}=\mathcal{O}(|\ln \varepsilon|), h_{\mathrm{ex}} \rightarrow+\infty$,

$$
\lambda^{1 / 4}|\ln \varepsilon| \rightarrow 0 \text { and } \delta \sqrt{h}_{\mathrm{ex}} \rightarrow 0
$$

and assume that Hypothesis (1.5) holds.

Let $d \in \mathbb{N}^{*}$ and let $\mathbf{D} \in \Lambda_{d}$ be a minimizer of the minimizing problem (7.2).

For $0<\varepsilon<1$, there exists $\left(v_{\varepsilon}, A_{\varepsilon}\right) \in \mathscr{H}$ which is in the Coulomb gauge with $d$ vortices of degree 1 s.t. $\operatorname{deg}\left(v_{\varepsilon}, p_{k}\right)=D_{k}$,

$$
\mathcal{F}\left(v_{\varepsilon}, A_{\varepsilon}\right)=h_{\mathrm{ex}}^{2} \mathbf{J}_{\mathbf{0}}+d M_{\Omega}\left[-h_{\mathrm{ex}}+H_{c_{1}}^{0}\right]+\mathscr{L}_{1}(d) \ln h_{\mathrm{ex}}+\mathscr{L}_{2}(d)+o(1)
$$

with $M_{\Omega}:=2 \pi\left\|\xi_{0}\right\|_{L^{\infty}(\Omega)}$ and

$$
H_{c_{1}}^{0}:=\frac{b^{2}|\ln \varepsilon|+\left(1-b^{2}\right)|\ln (\lambda \delta)|}{2\left\|\xi_{0}\right\|_{L^{\infty}(\Omega)}}+\widetilde{\gamma}_{b, \omega}
$$

where

$$
\widetilde{\gamma}_{b, \omega}:=\frac{\min _{\omega} W^{\text {micro }}+b^{2}[\gamma+\pi \ln b]}{2 \pi\left\|\xi_{0}\right\|_{L^{\infty}(\Omega)}},
$$

$\gamma$ is a universal constant defined in Lemma IX.1 of [4] and $W^{\text {micro }}$ is defined in Section 6.3.

Proposition 7.3 is proved in Appendix E. 


\section{Tool box}

The proof of the main theorems of this article is done in a classic way: by matching upper and lower bounds. A (sharp) upper bound is obtained by Proposition 7.3. Getting a sharp lower bound is the most challenging part of the proof. It needs the proof of several facts related with the vorticity defects of a family of quasi-minimizers (quantization, localization, size...).

In this section we present some technical and quite classical results adapted to our situation.

\subsection{An $\eta$-ellipticity property}

In this section we focus on quasi-minimizers. We let $h_{\mathrm{ex}}=\mathcal{O}(|\ln \varepsilon|)$ and we consider $\left\{\left(v_{\varepsilon}, A_{\varepsilon}\right) \mid 0<\varepsilon<1\right\}$ a family of quasi-minimizers for $\mathcal{F}$, i.e.,

$$
\mathcal{F}\left(v_{\varepsilon}, A_{\varepsilon}\right) \leqslant \inf _{\mathscr{H}} \mathcal{F}+o(1) .
$$

We assume that for all $\varepsilon \in(0,1),\left(v_{\varepsilon}, A_{\varepsilon}\right)$ is in the Coulomb gauge and that $v_{\varepsilon} \in H^{1}(\Omega, \mathbb{C})$ is s.t.

$$
\left\|\nabla\left|v_{\varepsilon}\right|\right\|_{L^{\infty}(\Omega)}=\mathcal{O}\left(\varepsilon^{-1}\right) .
$$

The major result of this section is a key tool in this article: an $\eta$ ellipticity property.

Proposition 8.1. - Let $h_{\mathrm{ex}}=\mathcal{O}(|\ln \varepsilon|)$ and let $\left\{\left(v_{\varepsilon}, A_{\varepsilon}\right) \mid 0<\varepsilon<1\right\} \subset$ $\mathscr{H}$ be a family in the Coulomb gauge satisfying (8.1) and (8.2).

For $\eta \in(0,1)$ there exist $\varepsilon_{\eta}>0$ and $C_{\eta}>0$ s.t. for $0<\varepsilon<\varepsilon_{\eta}$, if $z \in \Omega$ is s.t.

$$
b^{2} \int_{B(z, \sqrt{\varepsilon}) \cap \Omega}\left|\nabla v_{\varepsilon}\right|^{2}+\frac{b^{2}}{\varepsilon^{2}}\left(1-\left|v_{\varepsilon}\right|^{2}\right)^{2} \leqslant C_{\eta}|\ln \varepsilon|,
$$

then $\left|v_{\varepsilon}(z)\right|>\eta$.

Proposition 8.1 is proved in Appendix F.

By combining Proposition 8.1 with Theorem 5.6 we get immediately a first step in the (macroscopic) localization of the vorticity defects. In order to apply Theorem 5.6 we need assume

$$
\left\{\begin{array}{l}
\lambda, \delta \text { satisfy }(1.2), \delta^{2}|\ln \varepsilon| \rightarrow 0, h_{\mathrm{ex}} \rightarrow \infty, \\
(1.3) \text { holds for } h_{\mathrm{ex}} \text { with some } K \geqslant 0 \text { independent of } \varepsilon .
\end{array}\right.
$$


Corollary 8.2. - Assume that $\lambda, \delta$ and $h_{\mathrm{ex}}$ satisfy (8.3) and let $\left\{\left(v_{\varepsilon}, A_{\varepsilon}\right) \mid 0<\varepsilon<1\right\} \subset \mathscr{H}$ be s.t. (8.1) and (8.2) hold. There exist $0<\varepsilon_{0} \leqslant$ $\varepsilon_{K}$ and $M \geqslant 1$ s.t. for $0<\varepsilon<\varepsilon_{0}$, letting $\widetilde{\Lambda}_{\varepsilon}:=\Lambda \cap \bigcup_{d_{i} \neq 0} B\left(a_{i}, 2 \mathcal{M}_{K}|\ln \varepsilon|^{-s_{0}}\right)$ where the $\left(a_{i}, d_{i}\right)$ 's (depend on $\varepsilon$ ) are given by Proposition 5.5 and $\varepsilon_{K}, \mathcal{M}_{K}$ and $s_{0}$ are given by Theorem 5.6, we have

$$
\left\{\left|v_{\varepsilon}\right| \leqslant 1 / 2\right\} \subset \bigcup_{p \in \tilde{\Lambda}_{\varepsilon}} B\left(p, M|\ln \varepsilon|^{-\tilde{s}_{0}}\right) \text { where } \widetilde{s}_{0}:=\min \left\{s_{0}, 10\right\} .
$$

Proof. - We argue by contradiction and we assume that there exist $\varepsilon=$ $\varepsilon_{n} \downarrow 0$ and a sequence $\left(\left(v_{\varepsilon}, A_{\varepsilon}\right)\right)_{\varepsilon} \subset \mathscr{H}$ s.t. $(8.1)$ and (8.2) hold and s.t. for all $n$ there exists

$$
z_{0}=z_{0}^{n} \in\{|v| \leqslant 1 / 2\} \backslash \bigcup_{p \in \tilde{\Lambda}_{\varepsilon}} B\left(p, n|\ln \varepsilon|^{-\tilde{s}_{0}}\right) .
$$

Since (8.1) and (8.2) are gauge invariant we may assume that, for all $\varepsilon$, $\left(v_{\varepsilon}, A_{\varepsilon}\right)$ is in the Coulomb gauge.

Let $\mathcal{B}:=\left\{\left(B\left(a_{i}, r_{i}\right), d_{i}\right) \mid i \in \mathcal{J}\right\}$ be given by Proposition 5.5. Write $B_{i}:=B\left(a_{i}, r_{i}\right)$ for $i \in \mathcal{J}$. Note that by Theorem 5.6, from the quasiminimality of $\left(v_{\varepsilon}, A_{\varepsilon}\right)$, for $\varepsilon$ sufficiently small, we have $d_{i} \geqslant 0$ for all $i$ and $d:=\sum\left|d_{i}\right|=\sum d_{i}=\mathcal{O}(1)$. Up to a subsequence, we may thus assume that $d$ is independent of $\varepsilon$.

From the definition of $\widetilde{\Lambda}_{\varepsilon}$, we have

$$
\bigcup_{d_{i}>0} B_{i} \subset \bigcup_{p \in \tilde{\Lambda}} B\left(p, 2 \mathcal{M}_{K}|\ln \varepsilon|^{-s_{0}}\right) .
$$

Note that from Theorem 5.6 we have $\mathcal{F}\left(v_{\varepsilon}, 0\right)=\mathcal{O}\left(|\ln \varepsilon|^{2}\right)$. Then we may use Proposition 5.5 for the configuration $\left(v_{\varepsilon}, 0\right) \in \mathscr{H}$ to get a covering $\bigcup_{i \in \tilde{\mathcal{J}}} \widetilde{B}_{i}$ of $\left\{\left|v_{\varepsilon}\right|<1-|\ln \varepsilon|^{-2}\right\}$ with disjoint disks $\widetilde{B}_{i}=B\left(\widetilde{a}_{i}, \widetilde{r}_{i}\right), \sum \widetilde{r}_{i}<$ $|\ln \varepsilon|^{-10}$.

Therefore there is $\rho \in\left[2 \mathcal{M}_{K}|\ln \varepsilon|^{-\tilde{s}_{0}},\left(2 \mathcal{M}_{K}+6\right)|\ln \varepsilon|^{-\tilde{s}_{0}}\right]$ s.t.

$$
\left[\bigcup_{p \in \tilde{\Lambda}_{\varepsilon}} \partial B(p, \rho)\right] \cap\left[\bigcup_{i \in \mathcal{J}} B_{i} \cup \bigcup_{i \in \tilde{\mathcal{J}}} \widetilde{B}_{i}\right]=\emptyset \text {. }
$$

In particular $\left|v_{\varepsilon}\right| \geqslant 1-|\ln \varepsilon|^{-2}$ on $\bigcup_{p \in \tilde{\Lambda}_{\varepsilon}} \partial B(p, \rho)$. Thus, writing $\tilde{d}_{i}:=$ $\operatorname{deg}_{\partial \tilde{B}_{i}}\left(v_{\varepsilon}\right)$ when $\widetilde{B}_{i} \subset \Omega$, we get for $p \in \widetilde{\Lambda}_{\varepsilon}$

$$
\sum_{\tilde{B}_{i} \subset B(p, \rho)}\left|\widetilde{d}_{i}\right| \geqslant\left|\sum_{\tilde{B}_{i} \subset B(p, \rho)} \widetilde{d}_{i}\right|=\operatorname{deg}_{\partial B(p, \rho)}\left(v_{\varepsilon}\right)=\sum_{B_{i} \subset B(p, \rho)} d_{i} .
$$


Note that for sufficiently large $n$ we have $B\left(z_{0}, \sqrt{\varepsilon}\right) \cap \bigcup_{p \in \tilde{\Lambda}_{\varepsilon}} B(p, \rho)=\emptyset$.

On the other hand, since $\sum \widetilde{r}_{i}<|\ln \varepsilon|^{-10}$, we have for $\widetilde{B}_{i} \subset \Omega$ (here we use (5.14))

$$
F\left(v, \widetilde{B}_{i}\right) \geqslant \pi b^{2}\left|\widetilde{d}_{i}\right|(|\ln \varepsilon|-C \ln |\ln \varepsilon|) .
$$

Using Proposition 8.1 we obtain

$$
F(v) \geqslant\left(\pi b^{2} d+C_{1 / 2}\right)|\ln \varepsilon|-\mathcal{O}(\ln |\ln \varepsilon|)
$$

where $C_{1 / 2}>0$ is given by Proposition 8.1 with $\eta=1 / 2$. Estimate (8.4) is in contradiction with (5.32).

\subsection{Construction of the $\varepsilon^{s}$-bad discs}

As in the previous section we assume that $\lambda, \delta$ and $h_{\text {ex }}$ satisfy (8.3). In this section we establish the existence of $\varepsilon^{s}$-bad discs associated to a quasi-minimizing sequence. The construction of the bad discs requires the hypothesis: $\left|v_{\varepsilon}\right| \in W^{2,1}(\Omega)$.

An $\varepsilon^{s}$-bad discs family associated to a familly $\left\{\left(v_{\varepsilon}, A_{\varepsilon}\right) \mid 0<\varepsilon<1\right\} \subset \mathscr{H}$ consists in sets of discs that have small diameters (a roots of $\varepsilon$ ) s.t. for fix $\varepsilon$ the discs are "well separated", the union of the discs is a covering of $\{|v| \leqslant 1 / 2\}$ and each "heart" of a disc intersects $\{|v| \leqslant 1 / 2\}$. Such sets of discs give thus a nice visualization of $\{|v| \leqslant 1 / 2\}$.

In Section 9, adding an extra hypothesis on $\lambda, \delta$ and $h_{\text {ex }}$ we get some informations in terms of location and quantification of the $\varepsilon^{s}$-bad discs.

Proposition 8.3. - Assume that $\lambda, \delta$ and $h_{\mathrm{ex}}$ satisfy (8.3). There exists $M_{0} \in \mathbb{N}^{*}$ s.t. for $\mu \in(0,1 / 2)$, if $\left\{\left(v_{\varepsilon}, A_{\varepsilon}\right) \mid 0<\varepsilon<1\right\}$ is in the Coulomb gauge and agrees with (3.11) and (8.1), then there exist $\varepsilon_{\mu}>0$ and $C_{\mu} \geqslant 1$ (independent of $\varepsilon$ ) s.t. for $0<\varepsilon<\varepsilon_{\mu}$, there is $J_{\mu}=J_{\mu, \varepsilon} \subset\left\{1, \ldots, M_{0}\right\}$ (possibly empty) s.t. if $J_{\mu}=\emptyset$ then $|v|>1 / 2$ in $\Omega$ and if $J_{\mu} \neq \emptyset$ then there are $\left\{z_{i} \mid i \in J_{\mu}\right\} \subset \Omega$, a set of mutually distinct points, and $r \in\left[\varepsilon^{\mu}, \varepsilon^{\mu_{*}}\right]$ with $\mu_{*}:=2^{-M_{0}^{2}} \mu$ verifying:

(1) $\left|z_{i}-z_{j}\right| \geqslant r^{3 / 4}$ for $i, j \in J_{\mu}, i \neq j$,

(2) $\left\{\left|v_{\varepsilon}\right| \leqslant 1 / 2\right\} \subset \bigcup_{J_{\mu}} B\left(z_{i}, r\right) \subset \Omega$ and, for $i \in J_{\mu}, B\left(z_{i}, r / 4\right) \cap$ $\left\{\left|v_{\varepsilon}\right| \leqslant 1 / 2\right\} \neq \emptyset$,

(3) For $i \in J_{\mu}$ we have $r \int_{\partial B\left(z_{i}, r\right)}\left|\nabla v_{\varepsilon}\right|^{2}+\frac{1}{2 \varepsilon^{2}}\left(1-\left|v_{\varepsilon}\right|^{2}\right)^{2} \leqslant C_{\mu}$ and $|v| \geqslant 1-|\ln \varepsilon|^{-2}$ on $\partial B\left(z_{i}, r\right)$.

Proposition 8.3 is proved in Appendix G. We have the following standard estimate. 
Proposition 8.4. - Assume (8.3) and let $\left\{\left(v_{\varepsilon}, A_{\varepsilon}\right) \mid 0<\varepsilon<1\right\}$ be as in Proposition 8.3. Fix $\mu \in(0,1 / 2)$ and let $\varepsilon_{\mu}, C_{\mu}$ be given by Proposition 8.3. For $0<\varepsilon<\varepsilon_{\mu}$ we consider $J_{\mu},\left\{z_{i} \mid i \in J_{\mu}\right\} \subset \Omega$ and $r$ obtained in Proposition 8.3. We denote $d_{i}:=\operatorname{deg}_{\partial B\left(z_{i}, r\right)}\left(v_{\varepsilon}\right)$.

There exists $c_{\mu, b} \geqslant 1$ independent of $\varepsilon$ s.t. for $\varepsilon<\varepsilon_{\mu}$ we have

$$
\begin{gathered}
\left|d_{i}\right| \leqslant 4 \sqrt{C_{\mu}}, \\
\frac{1}{2} \int_{B\left(z_{i}, r\right)}\left|\nabla v_{\varepsilon}\right|^{2}+\frac{b^{2}}{2 \varepsilon^{2}}\left(1-\left|v_{\varepsilon}\right|^{2}\right)^{2} \geqslant \pi\left|d_{i}\right| \ln \left(\frac{r}{\varepsilon}\right)-c_{\mu, b}
\end{gathered}
$$

and then

$$
\begin{aligned}
F\left(v_{\varepsilon}, B\left(z_{i}, r\right)\right) \geqslant \pi\left|d_{i}\right| \inf _{B\left(z_{i}, r\right)} \alpha & {\left[\ln \left(\frac{r}{\varepsilon}\right)-c_{\mu, b}\right] } \\
& \geqslant \pi \inf _{B\left(z_{i}, r\right)} \alpha\left|d_{i}\right|\left[(1-\mu) \ln \varepsilon-c_{\mu, b}\right] .
\end{aligned}
$$

Moreover there is $0<\widetilde{\varepsilon}_{\mu} \leqslant \varepsilon_{\mu}$ s.t. for $0<\varepsilon<\widetilde{\varepsilon}_{\mu}$ we have

$$
d_{i} \neq 0 \text { for all } i
$$

and

$$
\sum_{i \in J_{\mu}}\left|d_{i}\right| \leqslant \mathcal{D}_{K, b}:=\frac{3 \mathcal{M}_{K}}{b^{2}}
$$

Proof. - It is classical to get (8.5) from Proposition 8.3(3) and the Cauchy Schwartz inequality. Estimate (8.6) follows from Proposition 8.3 and Lemma VI.1 in [2] and (8.7) is a consequence of (8.6).

The proof of (8.8) is done arguing by contradiction with the construction of a comparaison function

$$
\widetilde{v}:= \begin{cases}v & \text { in } \Omega \backslash B\left(z_{i_{0}}, r\right) \\ \widetilde{\rho} \mathrm{e}^{\tilde{\phi} \tilde{\phi}} & \text { in } B\left(z_{i_{0}}, r\right)\end{cases}
$$

s.t. $\widetilde{v} \in H^{1}(\Omega, \mathbb{C})$ and $F\left(\widetilde{v}, B\left(z_{i_{0}}, r\right)\right)=\mathcal{O}(1)$ where we assumed $d_{i_{0}}=0$.

Since $(v, A)$ is a quasi-minimizer of $\mathcal{F}$ we have $\mathcal{F}(v, A) \leqslant \mathcal{F}(\widetilde{v}, A)+o(1)$.

On the other hand, by direct calculations $\mathcal{F}(v, A)-\mathcal{F}(\widetilde{v}, A)=$ $F\left(v, B\left(z_{i_{0}}, r\right)\right)-F\left(\widetilde{v}, B\left(z_{i_{0}}, r\right)\right)+o(1)$. Consequently $F\left(v, B\left(z_{i_{0}}, r\right)\right)=\mathcal{O}(1)$ which is in contradiction with $F\left(v, B\left(z_{i_{0}}, r\right)\right) \geqslant C_{1 / 2}|\ln \varepsilon|$ (given by Proposition 8.1) for small $\varepsilon$.

We now prove (8.9). From (8.7) we have $\sum_{J_{\mu}}\left|d_{i}\right|\left[\pi(1-\mu)|\ln \varepsilon|-c_{\mu, b}\right] \leqslant$ $\frac{\mathcal{M}_{K}|\ln \varepsilon|}{b^{2}}$. Since $\mu \in(0,1 / 2)$, the last estimate gives the result for $\varepsilon>0$ sufficiently small. 


\subsection{Lower bounds in perforated disks}

The goal of this section is to get lower bounds for $\frac{1}{2} \int_{\mathcal{D}} \alpha|\nabla v|^{2}$ where $\mathcal{D}$ is a perforated disk s.t. $\mathcal{D} \subset \Omega$ and $|v| \geqslant 1 / 2$ in $\mathcal{D}$.

The starting point of the argument is an estimate on circles. Let $\widetilde{b} \in(0,1)$, $\beta \in L^{\infty}((0,2 \pi),[\widetilde{b}, 1])$. With Lemma D.7 in $[6]$, for $\varphi \in H^{1}((0,2 \pi), \mathbb{R})$ s.t. $\varphi(2 \pi)-\varphi(0)=2 \pi$, we have the following lower bound:

$$
\frac{1}{2} \int_{0}^{2 \pi} \beta\left|\partial_{\theta} \varphi\right|^{2} \geqslant \frac{2 \pi^{2}}{\int_{0}^{2 \pi} \frac{1}{\beta}} .
$$

In order to use (8.10) we need to do a preliminary analysis.

For $\alpha=U_{\varepsilon}^{2} \in L^{\infty}\left(\Omega,\left[b^{2}, 1\right]\right)$, using Lemma E.1 in [6], we have the existence of $C \geqslant 1$ (independent of $\varepsilon$ ) s.t.

$\left\{\right.$ For almost all $s \geqslant \delta / 3$, letting $\mathscr{C}_{s}$ be a circle with radius $s$,

we have $\int_{\mathscr{C}_{s} \cap \Omega}(1-\alpha) \leqslant C \lambda s$.

From now on, in all this section, we consider a sequence $\varepsilon=\varepsilon_{n} \downarrow 0$, $\lambda, \delta, h_{\mathrm{ex}}$ and $\left(\left(v_{\varepsilon}, A_{\varepsilon}\right)\right)_{\varepsilon} \subset \mathscr{H}$ satisfying the hypotheses of Proposition 8.3 (namely $(3.11),(8.1)$ and (8.3)). We drop the subscript $\varepsilon$ writing $(v, A)$ instead of $\left(v_{\varepsilon}, A_{\varepsilon}\right)$

Recall that $\eta_{\Omega}$ is defined in (6.14) and consider

$$
x_{\varepsilon} \in \Omega \text { and } 0<r=r_{\varepsilon}<R=R_{\varepsilon}<\eta_{\Omega} \text { s.t. } \operatorname{dist}\left(x_{\varepsilon}, \partial \Omega\right)>\eta_{\Omega}>0 \text {. }
$$

We then denote $\mathscr{R}:=B\left(x_{\varepsilon}, R\right) \backslash \overline{B\left(x_{\varepsilon}, r\right)} \subset \Omega$.

Assume $|v| \geqslant 1 / 2$ in $\mathscr{R}$ and let $d:=\operatorname{deg}_{\mathscr{R}}(v)$. From the proof of Proposition 8.3 (see (G.2) in Appendix G), there exists $1 / 2<t_{\varepsilon}<1, t_{\varepsilon}=1+o(1)$ s.t. $t_{\varepsilon} \in \operatorname{Im}(|v|) \cap\left[1-2 /|\ln \varepsilon|^{2}, 1-1 /|\ln \varepsilon|^{2}\right]$ and

$$
\left\{\begin{array}{l}
V\left(t_{\varepsilon}\right):=\left\{|v|=t_{\varepsilon}\right\} \text { is a finite union of Jordan curves included } \\
\text { in } \Omega \text { and of simple curves whose endpoints are on } \partial \Omega \text { and } \\
\mathcal{H}^{1}\left[V\left(t_{\varepsilon}\right)\right]=o(1) .
\end{array}\right.
$$

and since $\mathcal{H}^{2}\left(\left\{|v| \leqslant t_{\varepsilon}\right\}\right)=o(1)$ we then have

$$
\left\{\begin{array}{l}
\text { if } U \text { is a connected component of }\left\{|v| \leqslant t_{\varepsilon}\right\} \text { s.t. } \bar{U} \subset \Omega \\
\text { then there is } \Gamma, \text { a connected component of } V\left(t_{\varepsilon}\right), \\
\text { which is a Jordan curve s.t. } U \subset \operatorname{int}(\Gamma) .
\end{array}\right.
$$

Remark 8.5. - Since $\mathcal{H}^{1}\left[V\left(t_{\varepsilon}\right)\right]=o(1)$, for sufficiently small $\varepsilon$, if $\Gamma$ (resp. $U$ ) is a connected component of $V\left(t_{\varepsilon}\right)$ (resp. $\left\{|v| \leqslant t_{\varepsilon}\right\}$ ) which intersects $\mathscr{R}$ then $\Gamma$ is a Jordan curve (resp. $\partial U$ is a union of connected components of $\left.V\left(t_{\varepsilon}\right)\right)$. 
We have the following lemma:

LEMMA 8.6. - Assume $x_{\varepsilon}, r, R$ satisfy (8.12) and we assume $|v| \geqslant 1 / 2$ in $\mathscr{R}$. Then, for $s \in(r, R)$, letting

$$
K_{s}:=\left\{\theta \in[0,2 \pi)|| v\left(x_{\varepsilon}+s \mathrm{e}^{\imath \theta}\right) \mid \leqslant t_{\varepsilon}\right\}
$$

we have

$$
\mathcal{H}^{1}\left(K_{s}\right) \leqslant \pi \frac{\mathcal{H}^{1}\left[V\left(t_{\varepsilon}\right)\right]}{s} .
$$

Proof. - Let $s \in(r, R)$ be s.t. $\mathcal{H}^{1}\left(K_{s}\right)>0$ and denote $\widehat{\mathcal{K}}_{s}:=\left\{x_{\varepsilon}+\right.$ $\left.s \mathrm{e}^{\imath \theta} \mid \theta \in K_{s}\right\} \subset \partial B\left(x_{\varepsilon}, s\right)$. Then $\mathcal{H}^{1}\left(\widehat{\mathcal{K}}_{s}\right)=s \mathcal{H}^{1}\left(K_{s}\right)$.

On the one hand, letting $\mathcal{V}_{\mathscr{R}}\left(t_{\varepsilon}\right)$ be the union of the connected components of $\left\{|v| \leqslant t_{\varepsilon}\right\}$ which intersect $\mathscr{R}$, we have $\widehat{\mathcal{K}}_{s}=\mathcal{V}_{\mathscr{R}}\left(t_{\varepsilon}\right) \cap \partial B\left(x_{\varepsilon}, s\right)$.

On the other hand, by Remark 8.5, $\partial \mathcal{V}_{\mathscr{R}}\left(t_{\varepsilon}\right)$ is a union of connected components of $V\left(t_{\varepsilon}\right)$ which are Jordan curves. Among these Jordan curves, we may select the maximal curves w.r.t. the inclusion of their interior. We denote these maximal curves by $\Gamma_{1}, \ldots, \Gamma_{N}$ and we let for $i \in\{1, \ldots, N\}, \mathcal{V}_{i}:=$ $\overline{\operatorname{int}\left(\Gamma_{i}\right)}$. We then obtain $\mathcal{V}_{\mathscr{R}}\left(t_{\varepsilon}\right) \subset \bigcup_{i=1}^{N} \mathcal{V}_{i}$ and thus $\widehat{\mathcal{K}}_{s} \subset \bigcup_{i=1}^{N}\left[\partial B\left(x_{\varepsilon}, s\right) \cap\right.$ $\left.\mathcal{V}_{i}\right]$

For $i \in\{1, \ldots, N\}$, we fix $x_{i} \in \mathcal{V}_{i}$ and we define the disk $B_{i}:=$ $\overline{B\left(x_{i}, \operatorname{diam}\left(\mathcal{V}_{i}\right)\right)}$. It is clear that $\mathcal{V}_{i} \subset B_{i}$. Consequently

$$
\mathcal{H}^{1}\left[\partial B\left(x_{\varepsilon}, s\right) \cap \mathcal{V}_{i}\right] \leqslant \mathcal{H}^{1}\left[\partial B\left(x_{\varepsilon}, s\right) \cap B_{i}\right] \leqslant 2 \pi \operatorname{diam}\left(\mathcal{V}_{i}\right) .
$$

We claim that $2 \operatorname{diam}\left(\mathcal{V}_{i}\right) \leqslant \mathcal{H}^{1}\left(\Gamma_{i}\right)$. Since the curves $\Gamma_{i}$ are pairwise disjoint, we have $\sum_{i=1}^{N} \mathcal{H}^{1}\left(\Gamma_{i}\right) \leqslant \mathcal{H}^{1}\left[V\left(t_{\varepsilon}\right)\right]$.

We may now conclude:

$$
\begin{aligned}
s \mathcal{H}^{1}\left(K_{s}\right)=\mathcal{H}^{1}\left(\widehat{\mathcal{K}}_{s}\right) \leqslant \sum_{i=1}^{N} \mathcal{H}^{1}\left[\partial B\left(x_{\varepsilon}, s\right)\right. & \left.\cap \mathcal{V}_{i}\right] \\
& \leqslant \pi \sum_{i=1}^{N} 2 \operatorname{diam}\left(\mathcal{V}_{i}\right) \leqslant \pi \mathcal{H}^{1}\left[V\left(t_{\varepsilon}\right)\right]
\end{aligned}
$$

The next proposition is one of the major uses of the dilution $\lambda \rightarrow 0$.

Proposition 8.7. - Let $x_{\varepsilon}, r, R$ satisfying (8.12) and $r=o(R)$, assume also $|v| \geqslant 1 / 2$ in $\mathscr{R}$. We write $d:=\operatorname{deg}_{\mathscr{R}}(v)$ and, in $\mathscr{R}$, we let $w:=v /|v|$ and $\rho:=|v|$.

(1) If $r \geqslant \delta / 3$ and if $\mathcal{H}^{1}\left[V\left(t_{\varepsilon}\right)\right] / r+\left(1-t_{\varepsilon}^{2}\right)+\lambda=o[1 / \ln (R / r)]$ then

$$
\frac{1}{2} \int_{\mathscr{R}} \alpha|\nabla v|^{2} \geqslant \frac{1}{2} \int_{\mathscr{R}} \alpha \rho^{2}|\nabla w|^{2} \geqslant \pi d^{2}\left[\ln \left(\frac{R}{r}\right)-o(1)\right] .
$$


(2) If $r=o(1)$ and if $\mathcal{H}^{1}\left[V\left(t_{\varepsilon}\right)\right] / r+\left(1-t_{\varepsilon}^{2}\right)=o[1 / \ln (R / r)]$ then

$$
\frac{1}{2} \int_{\mathscr{R}}|\nabla v|^{2} \geqslant \frac{1}{2} \int_{\mathscr{R}} \rho^{2}|\nabla w|^{2} \geqslant \pi d^{2}\left[\ln \left(\frac{R}{r}\right)-o(1)\right] .
$$

Proof. - We prove the first assertion. We claim that, up to replacing $v$ with $\underline{v}$, we may assume $|v| \leqslant 1$ in $\Omega$. Moreover, if $d=0$ then there is nothing to prove. We then assume $d \neq 0$.

We write $v=\rho \mathrm{e}^{\imath d \varphi}$ where $\varphi$ is locally defined and its gradient is globally defined. Letting $x_{\varepsilon}+\mathbb{R}^{+}:=\left\{x_{\varepsilon}+s \mid s \geqslant 0\right\}$, we may assume $\varphi \in H^{1}\left(\mathscr{R} \backslash\left(x_{\varepsilon}+\right.\right.$ $\left.\left.\mathbb{R}^{+}\right), \mathbb{R}\right)$. For $s \in(r, R)$, we let $\varphi_{s}(\theta)=\varphi\left(x_{\varepsilon}+s \mathrm{e}^{\imath \theta}\right), \rho_{s}(\theta)=\left|v\left(x_{\varepsilon}+s \mathrm{e}^{\imath \theta}\right)\right|$ and $\alpha_{s}(\theta)=\alpha\left(x_{\varepsilon}+s \mathrm{e}^{\imath \theta}\right)$. Then $\varphi_{s} \in H^{1}((0,2 \pi), \mathbb{R})$ is s.t. $\varphi_{s}(2 \pi)-\varphi_{s}(0)=2 \pi$ and we immediately get

$$
\frac{1}{2} \int_{\mathscr{R}} \alpha \rho^{2}|\nabla w|^{2} \geqslant \frac{d^{2}}{2} \int_{r}^{R} \frac{\mathrm{d} s}{s} \int_{0}^{2 \pi} \alpha_{s} \rho_{s}^{2}\left|\partial_{\theta} \varphi_{s}\right|^{2} \mathrm{~d} \theta .
$$

From (8.10) with $\beta:=\alpha_{s} \rho_{s}^{2}$ we get

$$
\frac{1}{2} \int_{0}^{2 \pi} \alpha_{s} \rho_{s}^{2}\left|\partial_{\theta} \varphi_{s}\right|^{2} \geqslant \frac{2 \pi^{2}}{\int_{0}^{2 \pi} \frac{1}{\alpha_{s} \rho_{s}^{2}}} .
$$

Since $b^{2} / 4 \leqslant \alpha_{s} \rho_{s}^{2} \leqslant 1$ we have

$0 \leqslant\left(\int_{0}^{2 \pi} \frac{1}{\alpha_{s} \rho_{s}^{2}}\right)-2 \pi=\int_{0}^{2 \pi} \frac{1-\alpha_{s} \rho_{s}^{2}}{\alpha_{s} \rho_{s}^{2}} \leqslant \frac{4}{b^{2}}\left(\int_{0}^{2 \pi} 1-\rho_{s}^{2}+\int_{0}^{2 \pi} 1-\alpha_{s}\right)$.

On the one hand, from Lemma 8.6 we have

$$
\int_{0}^{2 \pi} 1-\rho_{s}^{2} \leqslant \mathcal{H}^{1}\left(K_{s}\right)+\left[2 \pi-\mathcal{H}^{1}\left(K_{s}\right)\right]\left(1-t_{\varepsilon}^{2}\right) \leqslant \frac{\pi \mathcal{H}^{1}\left[V\left(t_{\varepsilon}\right)\right]}{s}+2 \pi\left(1-t_{\varepsilon}^{2}\right) .
$$

On the other hand, using (8.11), there is $C \geqslant 1$ (independent of $\varepsilon$ ) s.t. $\int_{0}^{2 \pi} 1-\alpha_{s} \leqslant C \lambda$. Then

$$
\int_{0}^{2 \pi} \frac{1}{\alpha_{s} \rho_{s}^{2}} \leqslant 2 \pi+\frac{4}{b^{2}}\left[\frac{\pi \mathcal{H}^{1}\left[V\left(t_{\varepsilon}\right)\right]}{s}+2 \pi\left(1-t_{\varepsilon}^{2}\right)+C \lambda\right] .
$$

We thus get

$$
\begin{aligned}
\frac{1}{2} \int_{\mathscr{R}} \alpha \rho^{2}|\nabla w|^{2} & \geqslant d^{2} \int_{r}^{R} \frac{\mathrm{d} s}{s} \frac{2 \pi^{2}}{2 \pi-\frac{4}{b^{2}}\left[\pi \mathcal{H}^{1}\left[V\left(t_{\varepsilon}\right)\right] / s+2 \pi\left(1-t_{\varepsilon}^{2}\right)+C \lambda\right]} \\
& =\pi d^{2}\left[\ln \left(\frac{R}{r}\right)+o(1)\right] .
\end{aligned}
$$

The second assertion is obtained exactly in the same way than the first one. Indeed, since $\alpha$ plays no role in the statement, we may use the same argumentation with $\lambda=0$ and $\delta>0$ an arbitrary small number. 
We now state the reformulation of Proposition 8.7 by replacing the annular $\mathscr{R}$ with a perforated disk.

Corollary 8.8. - Let $D_{0} \in \mathbb{N}^{*}$ be independent of $\varepsilon, 0<r=r_{\varepsilon}<R=$ $R_{\varepsilon}$ be s.t. $r=o(R), N=N_{\varepsilon} \in \mathbb{N}^{*}$ be s.t. $N \leqslant D_{0}$ and $z_{1}=z_{1}^{\varepsilon}, \ldots, z_{N}=z_{N}^{\varepsilon}$ be s.t. $\left|z_{i}-z_{j}\right| \geqslant 8 r$ for $i \neq j$.

Let $y=y_{\varepsilon} \in \Omega$ and assume $z_{1}, \ldots, z_{N} \in B(y, R) \subset B(y, 4 R) \subset B\left(y, \eta_{\Omega}\right) \subset$ $\Omega$. We let $\mathcal{D}:=B(y, 2 R) \backslash \bigcup_{i=1}^{N} \overline{B\left(z_{i}, r\right)}$.

Assume $\rho=|v| \geqslant 1 / 2$ in $\mathcal{D}$. For $i \in\{1, \ldots, N\}$, we let $d_{i}:=\operatorname{deg}_{\partial B\left(z_{i}, r\right)}(v)$. We also assume $d_{i}>0$ for all $i \in\{1, \ldots, N\}$ and $\sum_{i=1}^{N} d_{i} \leqslant D_{0}$. Write $v=\rho w$ in $\mathcal{D}$.

Then there exists $C_{0}>0$ depending only on $D_{0}$ s.t.:

(1) If $r \geqslant \delta / 3$ and $\mathcal{H}^{1}\left[V\left(t_{\varepsilon}\right)\right] / r+\left(1-t_{\varepsilon}^{2}\right)+\lambda=o[1 / \ln (R / r)]$ then, for sufficiently small $\varepsilon$, we have

$$
\frac{1}{2} \int_{\mathcal{D}} \alpha|\nabla v|^{2} \geqslant \frac{1}{2} \int_{\mathcal{D}} \alpha \rho^{2}|\nabla w|^{2} \geqslant \pi \sum_{i=1}^{N} d_{i}^{2} \ln (R / r)-C_{0} .
$$

(2) If $\mathcal{H}^{1}\left[V\left(t_{\varepsilon}\right)\right] / r+\left(1-t_{\varepsilon}^{2}\right)=o[1 / \ln (R / r)]$ then, for sufficiently small $\varepsilon$, we have

$$
\frac{1}{2} \int_{\mathcal{D}}|\nabla v|^{2} \geqslant \frac{1}{2} \int_{\mathcal{D}} \rho^{2}|\nabla w|^{2} \geqslant \pi \sum_{i=1}^{N} d_{i}^{2} \ln (R / r)-C_{0} .
$$
in $\Omega$.

Proof. - We claim that, up to replacing $v$ with $\underline{v}$, we may assume $|v| \leqslant 1$

We first proceed to a scaling with the conformal mapping:

$$
\begin{aligned}
\Phi: B(y, 4 R) & \longrightarrow B(0,4) \\
x & \longmapsto \frac{x-y}{R} .
\end{aligned}
$$

We then let $\widehat{z}_{i}:=\Phi\left(z_{i}\right), \widehat{r}:=r / R, \widehat{\mathcal{D}}:=\Phi[\mathcal{D}]=B(0,2) \backslash \bigcup_{i=1}^{N} \overline{B\left(\widehat{z}_{i}, \widehat{r}\right)}$, $\widehat{\alpha}:=\alpha \circ \Phi^{-1}$ and $\widehat{v}:=v \circ \Phi^{-1}$.

If $N=1$ or $N \geqslant 2$ and $\left|\widehat{z}_{i}-\widehat{z}_{j}\right| \geqslant 4 \times 10^{-2 D_{0}}$ for $i \neq j$ then, letting $\widetilde{\Omega}:=B(0,4), \eta_{\tilde{\Omega}}=10^{-1}$, we may apply Proposition $8.7(1)$

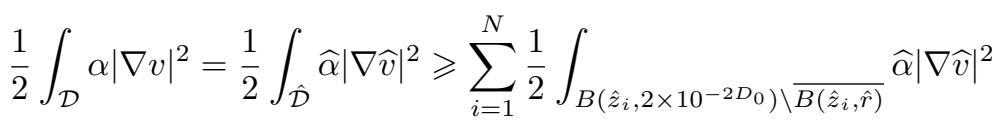

$$
\begin{aligned}
& \geqslant \pi \sum_{i=1}^{N} d_{i}^{2}\left(|\ln (R / r)|-\left|\ln \left(2 \times 10^{-2 D_{0}}\right)\right|\right)+o(1) \text {. }
\end{aligned}
$$


This estimate is the desired result with $C_{0}=\pi D_{0}^{2}\left|\ln \left(2 \times 10^{-2 D_{0}}\right)\right|+1$.

If we are not in the previous case, i.e. $N \geqslant 2$ and there exists $i \neq j$ s.t. $\left|\widehat{z}_{i}-\widehat{z}_{j}\right|<4 \times 10^{-2 D_{0}}$, then we apply the separation process presented in $[6$, Section C.3.1] to the domain $\widehat{\mathcal{D}}$ with $\eta_{\text {stop }}:=10^{-2 D_{0}}$.

The key ingredient in the separation process is a variant of Theorem IV.1 in [4] (stated with $P=9$, the general case $P \in \mathbb{N} \backslash\{0,1\}$ is left to the reader):

Lemma 8.9. - Let $N \geqslant 2, P \in \mathbb{N} \backslash\{0,1\}, x_{1}, \ldots, x_{N} \in \mathbb{R}^{2}$ and $\eta>0$. There are $\kappa \in\left\{P^{0}, \ldots, P^{N-1}\right\}$ and $\emptyset \neq J \subset\{1, \ldots, N\}$ s.t.

$$
\bigcup_{i=1}^{N} B\left(x_{i}, \eta\right) \subset \bigcup_{i \in J} B\left(x_{i}, \kappa \eta\right) \text { and }\left|x_{i}-x_{j}\right| \geqslant(P-1) \kappa \eta \text { for } i, j \in J, i \neq j .
$$

The separation process is an iterative selection of points in $\left\{\widehat{z}_{1}, \ldots, \widehat{z}_{N}\right\}$ associated to the construction of a good radius.

We initialize the process by letting $\eta_{0}:=\widehat{r}, M_{0}:=N$ and $J_{0}=\left\{1, \ldots, M_{0}\right\}$.

For $k \geqslant 1$ (where $k$ is the index in the iterative process) we construct a set $\emptyset \neq J_{k} \subsetneq J_{k-1}, M_{k}:=\operatorname{Card}\left(J_{k}\right)$ and 3 numbers

$$
\kappa_{k} \in\left\{9^{1}, \ldots, 9^{M_{k-1}-1}\right\}, \quad \eta_{k}^{\prime}:=\frac{1}{4} \min _{\substack{i, j \in J_{k-1} \\ i \neq j}}\left|\widehat{z}_{i}-\widehat{z}_{j}\right| \text { and } \eta_{k}:=2 \kappa_{k} \eta_{k}^{\prime} .
$$

These objects are obtained with Lemma 8.9 with $P=9, N=M_{k-1}=$ $\operatorname{Card}\left(J_{k-1}\right),\left\{x_{1}, \ldots, x_{N}\right\}=\left\{z_{i} \mid i \in J_{k-1}\right\}, J=J_{k}, \eta=\eta_{k}, \kappa=\kappa_{k}$ The process stops at the end of Step $K_{0} \geqslant 1$ if $M_{K_{0}}=1$ or $M_{K_{0}} \geqslant 2$ and $\min _{\substack{i, j \in J_{K_{0}} \\ i \neq j}}\left|\widehat{z}_{i}-\widehat{z}_{j}\right|>4 \eta_{\text {stop }}$.

By construction, we have for $1 \leqslant k \leqslant K_{0}, \emptyset \neq J_{k} \subsetneq J_{k-1}$ and $\eta_{k-1} \leqslant$ $\eta_{k}^{\prime}<\eta_{k}$. In particular, since $\operatorname{Card}\left(J_{0}\right) \leqslant D_{0}$, we get $K_{0} \leqslant D_{0}-1$.

By definition, for $k \in\left\{1, \ldots, K_{0}\right\}$ we have $2 \cdot 9 \eta_{k}^{\prime} \leqslant \eta_{k} \leqslant 9^{D_{0}} \eta_{k}^{\prime}$. We let

$$
\eta_{0}:= \begin{cases}9^{D_{0}} \cdot \eta_{\text {stop }} & \text { if } M_{K_{0}}=1 \\ \min \left\{9^{D_{0}} \cdot \eta_{\text {stop }}, \frac{1}{4} \min _{i, j \in J_{K_{0}}, i \neq j}\left|\widehat{z}_{i}-\widehat{z}_{j}\right|\right\} & \text { if } M_{K_{0}} \geqslant 2\end{cases}
$$

and then $\eta_{0} \geqslant \eta_{\text {stop }}=10^{-2 D_{0}}$. For $k \in\left\{0, \ldots, K_{0}-1\right\}$ and $i \in J_{k}$ we denote $\mathscr{R}_{i, k}:=B\left(\widehat{z}_{i}, \eta_{k+1}^{\prime}\right) \backslash \overline{B\left(\widehat{z}_{i}, \eta_{k}\right)}$, and, for $i \in J_{K_{0}}, \mathscr{R}_{i}:=B\left(\widehat{z}_{i}, \eta_{0}\right) \backslash$ $\overline{B\left(\widehat{z}_{i}, \eta_{K_{0}}\right)}$. By construction, the previous rings are pairwise disjoint. From 
Proposition $8.7(1)$ we have for $k \in\left\{0, \ldots, K_{0}-1\right\}$ and $i \in J_{k}$ :

$$
\begin{aligned}
\frac{1}{2} \int_{\mathscr{R}_{i, k}} \widehat{\alpha}|\nabla \widehat{v}|^{2} & \geqslant \pi \operatorname{deg}_{\mathscr{R}_{i, k}}(\widehat{v})^{2}\left[\ln \left(\eta_{k+1} / \eta_{k}\right)-\ln \left(9^{D_{0}}\right)\right]-o(1) \\
& \geqslant \pi \sum_{\hat{z}_{j} \in B\left(\hat{z}_{i}, \eta_{k+1}^{\prime}\right)} d_{j}^{2} \ln \left(\eta_{k+1} / \eta_{k}\right)-\pi D_{0}^{2} \ln \left(9^{D_{0}}\right)-o(1) .
\end{aligned}
$$

And for $i \in J_{K_{0}}$ :

$$
\begin{aligned}
\frac{1}{2} \int_{\mathscr{R}_{i}} \widehat{\alpha}|\nabla \widehat{v}|^{2} & \geqslant \pi \operatorname{deg}_{\mathscr{R}_{i}}(\widehat{v})^{2} \ln \left(\eta_{0} / \eta_{K_{0}}\right)-o(1) \\
& \geqslant \pi \sum_{\hat{z}_{j} \in B\left(\hat{z}_{i}, \eta_{0}\right)} d_{j}^{2} \ln \left(\eta / \eta_{K_{0}}\right)-o(1) .
\end{aligned}
$$

By summing the previous lower bound we get the result. As for Proposition 8.7, the second assertion is obtained in a similar way than the first assertion.

\subsection{Lower bounds in a perforated domain}

In this section we state a lower bound for a weighted Dirichlet energy in the domain $\Omega$ perforated by small (but not too small) disks. The philosophy of this lower bound is that in the case which interest us we may ignore the weight if the perforations are not too small; it is an effect of the dilution $\lambda \rightarrow 0$.

Proposition 8.10. - Let $\beta \in(0,1),\left(\widetilde{\alpha}_{n}\right)_{n} \subset L^{\infty}\left(\Omega,\left[\beta^{2}, 1\right]\right)$ be s.t.

$$
K_{n}:=\sqrt{\int_{\Omega}\left(1-\widetilde{\alpha}_{n}\right)^{2}} \rightarrow 0 .
$$

Let $N \in \mathbb{N}^{*}$ and $(\mathbf{z}, \mathbf{d})=(\mathbf{z}, \mathbf{d})^{(n)} \subset\left(\Omega^{N}\right)^{*} \times \mathbb{Z}^{N}$ be s.t. $\mathbf{D}$ is independant of $n$. We denote $\hbar:=\min _{i} \operatorname{dist}\left(z_{i}, \partial \Omega\right)$.

Assume the existence of $\widetilde{r}>0$ s.t. $\tilde{r}=o(1),(6.1)$ holds and s.t. there is $C_{1}>0$ (independent of $n$ ) satisfying $\frac{\tilde{r}|\ln \tilde{r}|}{\hbar} \leqslant C_{1}$. Write $\Omega_{\tilde{r}}:=\Omega \backslash \bigcup \overline{B\left(z_{i}, \widetilde{r}\right)}$.

Let $\left(u_{n}\right)_{n} \subset H^{1}(\Omega, \mathbb{C})$ satisfying $\left|u_{n}\right| \geqslant \frac{1}{2}$ in $\Omega_{\tilde{r}}$ and $\operatorname{deg}_{\partial B\left(z_{i}, \tilde{r}\right)}\left(u_{n}\right)=d_{i}$ for all $i$.

Assume also

$$
L_{n}:=\sqrt{\int_{\Omega_{\tilde{r}}}\left(1-\left|u_{n}\right|^{2}\right)^{2}} \rightarrow 0 .
$$


Then

$$
\begin{aligned}
\int_{\Omega_{\tilde{r}}} \widetilde{\alpha}_{n} \mid \nabla & \left.u_{n}\right|^{2} \geqslant \int_{\Omega_{\tilde{r}}}\left|\nabla \Phi_{\star}^{(\mathbf{z}, \mathbf{d})}\right|^{2} \\
& -\left(4 \beta^{-1}+3\right)\left\|\nabla \Phi_{\star}^{(\mathbf{z}, \mathbf{d})}\right\|_{L^{\infty}\left(\Omega_{\tilde{r})}\right.}\left\|\nabla \Phi_{\star}^{(\mathbf{z}, \mathbf{d})}\right\|_{L^{2}\left(\Omega_{\tilde{r}}\right)}\left(K_{n}+L_{n}\right)-\mathcal{O}(X)
\end{aligned}
$$

with $\Phi_{\star}^{(\mathbf{z}, \mathbf{d})}$ is defined in Remark 6.3 and $X$ is defined in (6.8).

Proposition 8.10 is proved in Appendix H.

\section{Study of the $\varepsilon^{s}$-bad discs}

In this section, in addition to the assumption (8.3) on $\lambda, \delta$ and $h_{\mathrm{ex}}$, we assume that (1.4) holds. This (technical) hypothesis (1.4) is a little bit more restrictive than $(7.3)\left(\delta \sqrt{h_{\mathrm{ex}}} \rightarrow 0\right)$ used to get a nice upper bound.

Let $\varepsilon=\varepsilon_{n} \downarrow 0$ and let $((v, A))_{\varepsilon}=\left(\left(v_{\varepsilon}, A_{\varepsilon}\right)\right)_{\varepsilon}$ be a sequence that agrees with (3.11) and (8.1). Let also $\mu \in(0,1 / 2)$.

Since (3.11) and (8.1) are gauge invariant we may assume that $(v, A)$ is in the Coulomb gauge.

The goal of this section is to prove that, for sufficiently small $\varepsilon$ and $\mu$, if $J_{\mu} \neq \emptyset$ then $d_{i}=1, \operatorname{dist}\left(z_{i}, \Lambda\right) \leqslant \ln \left(h_{\mathrm{ex}}\right) / \sqrt{h_{\mathrm{ex}}}$ and $z_{i} \in \omega_{\varepsilon}$ for all $i \in J_{\mu}$ and for $i \neq j,\left|z_{i}-z_{j}\right| \geqslant \ln \left(h_{\mathrm{ex}}\right) / h_{\mathrm{ex}}$ with a "uniform" distribution of the $z_{i}$ 's around $\Lambda$.

With the notation of Proposition 8.3 we let $\Omega_{r}:=\Omega \backslash \bigcup_{i \in J_{\mu}} \overline{B\left(z_{i}, r\right)}$ and $d:=\sum_{i \in J_{\mu}}\left|d_{i}\right|$.

In view of the goal of this section we may argue on subsequences. First note that from (8.8) we have $d_{i} \neq 0$ for all $i$. Up to a subsequence, from (8.9), we may assume that $J_{\mu} \neq \emptyset$ and independent of $\varepsilon$ as well as the $d_{i}$ 's.

Since we are interested here only in informations related with $|v|$ and the $d_{i}$ 's, we may consider that $(v, A)$ is in the Coulomb gauge and we may also change the potential vector. Namely, we may assume that $A=\nabla^{\perp} \xi$ with $\xi=\xi_{\varepsilon} \in H_{0}^{1} \cap H^{2}(\Omega, \mathbb{R})$ is the unique solution of (5.23). Note that (8.1) still holds.

Consequently, $\operatorname{curl}(A) \in H^{1}$ and then with (3.5) and (5.5): $\|\xi\|_{H^{3}(\Omega)} \leqslant$ $C|\ln \varepsilon|$.

From Proposition 5.7 and letting $\zeta=\zeta_{\varepsilon}:=\xi-h_{\mathrm{ex}} \xi_{0}$

$$
\mathcal{F}\left(v, \nabla^{\perp} \xi\right)=h_{\text {ex }}^{2} \mathbf{J}_{\mathbf{0}}+F(v)+2 \pi h_{\mathrm{ex}} \sum d_{i} \xi_{0}\left(z_{i}\right)+\widetilde{V}_{(\mathbf{z}, \mathbf{d})}(\zeta)+o(1) .
$$


Proposition 5.13 implies $\widetilde{V}_{(\mathbf{z}, \mathbf{d})}(\zeta)=\mathcal{O}(1)$. Consequently

$$
\mathcal{F}\left(v, \nabla^{\perp} \xi\right)=h_{\mathrm{ex}}^{2} \mathbf{J}_{\mathbf{0}}+F(v)+2 \pi h_{\mathrm{ex}} \sum d_{i} \xi_{0}\left(z_{i}\right)+\mathcal{O}(1) .
$$

In particular we have $\mathcal{F}\left(v, \nabla^{\perp} \xi\right) \leqslant h_{\text {ex }}^{2} \mathbf{J}_{\mathbf{0}}+o(1)$, thus with (9.1) we get

$$
F(v) \leqslant-2 \pi h_{\mathrm{ex}} \sum d_{i} \xi_{0}\left(z_{i}\right)+\mathcal{O}(1) .
$$

From Corollary 8.2 and Propositions 8.3 and 8.4 we deduce $-\sum d_{i} \xi_{0}\left(z_{i}\right)=$ $\left\|\xi_{0}\right\|_{L^{\infty}(\Omega)} \sum d_{i}+o(1)$ and we immediately obtain

$$
\sum d_{i} \geqslant 0
$$

On the other hand, from Proposition 7.3, we have

$$
\mathcal{F}\left(v, \nabla^{\perp} \xi\right) \leqslant h_{\mathrm{ex}}^{2} \mathbf{J}_{\mathbf{0}}+d M_{\Omega}\left[-h_{\mathrm{ex}}+H_{c_{1}}^{0}\right]+\mathscr{L}_{1}(d) \ln h_{\mathrm{ex}}+\mathcal{O}(1) .
$$

By combining (9.1) and (9.4) we get

$$
F(v) \leqslant d \pi\left[b^{2}|\ln \varepsilon|+\left(1-b^{2}\right)|\ln (\lambda \delta)|\right]+\mathscr{L}_{1}(d) \ln h_{\mathrm{ex}}+\mathcal{O}(1) .
$$

In conclusion, from (8.6) in conjunction with (9.5) we obtain

$$
\frac{1}{2} \int_{\Omega_{r}} \alpha|\nabla v|^{2} \leqslant d \pi\left[b^{2}|\ln r|+\left(1-b^{2}\right)|\ln (\lambda \delta)|\right]+\mathscr{L}_{1}(d) \ln h_{\mathrm{ex}}+\mathcal{O}(1) .
$$

We first have the following proposition.

Proposition 9.1. - Assume

$$
0<\mu<\min \left\{\frac{1}{\mathcal{D}_{K, b}+1}, \frac{1-b^{2}}{2\left(\mathcal{D}_{K, b}+1\right)}\right\}
$$

where $\mathcal{D}_{K, b}=\frac{3 \mathcal{M}_{K}}{b^{2}}$ and $\mathcal{M}_{K}$ is as in Theorem 5.6.

Then there exists $\widetilde{\varepsilon}_{\mu}>0$ s.t. for $0<\varepsilon<\widetilde{\varepsilon}_{\mu}$ if $J_{\mu} \neq \emptyset$ then

(1) $d_{i}>0$ for all $i$,

(2) $\operatorname{dist}\left(z_{i}, \omega_{\varepsilon}\right)<\sqrt{\varepsilon}$.

\section{Proof.}

Step 1. We prove that $d_{i}>0$ for all $i$. - We argue by contradiction and we assume the existence of an extraction still denoted by $\varepsilon=\varepsilon_{n} \downarrow 0$ s.t. $J_{-}:=\left\{i \in J_{\mu} \mid d_{i}<0\right\} \neq \emptyset$ (from (8.8), for $0<\varepsilon<\widetilde{\varepsilon}_{\mu}$, we have $d_{i} \neq 0$ for all $\left.i \in J_{\mu}\right)$. 
From (9.3) we thus obtain: $\sum_{i \in J_{\mu} \backslash J_{-}} d_{i} \geqslant d+1$. Then, with the help of (8.7), we obtain

$$
\begin{aligned}
F(v) & \geqslant b^{2}(1-\mu) \pi|\ln \varepsilon|\left(\sum_{i \in J_{-}}\left|d_{i}\right|+\sum_{i \in J_{\mu} \backslash J_{-}} d_{i}\right) \\
& \geqslant(d+2) \pi(1-\mu) b^{2}|\ln \varepsilon|+\mathcal{O}(1) .
\end{aligned}
$$

Consequently $(9.5)$ implies $d(1+o(1)) \geqslant(d+2)(1-\mu)-o(1)$. This inequality gives $\mu \geqslant \frac{2}{d+2}-o(1)$ which is in contradiction with $0<\mu<\left(\mathcal{D}_{K, b}+1\right)^{-1}$ for sufficiently small $\varepsilon>0$ (here we used $\mathcal{D}_{K, b} \geqslant \mathcal{M}_{K} \geqslant d$ ).

Step 2. We prove that $\operatorname{dist}\left(z_{i}, \omega_{\varepsilon}\right)<\sqrt{\varepsilon}$ for all $i$. - We argue by contradiction and we assume the existence of a subsequence still denoted by $\varepsilon=$ $\varepsilon_{n} \downarrow 0$ and $i_{0} \in J_{\mu}$ s.t. $\operatorname{dist}\left(z_{i_{0}}, \omega_{\varepsilon}\right) \geqslant \sqrt{\varepsilon}$. From (5.8) we have $\inf _{B\left(z_{i_{0}}, r\right)} \alpha \geqslant$ $1-o\left(|\ln \varepsilon|^{-2}\right)$. Consequently using (8.7) we get $F\left(v, B\left(z_{i_{0}}, r\right)\right) \geqslant d_{i_{0}} \pi(1-$ $\mu)|\ln \varepsilon|-\mathcal{O}(1)$. Then $F(v) \geqslant \pi b^{2}(1-\mu) d|\ln \varepsilon|+\pi\left(1-b^{2}\right)(1-\mu) d_{i_{0}}|\ln \varepsilon|-\mathcal{O}(1)$.

From (9.5) we obtain

$$
d b^{2}|\ln \varepsilon|+\mathcal{O}(\ln |\ln \varepsilon|) \geqslant b^{2}(1-\mu) d|\ln \varepsilon|+\left(1-b^{2}\right)(1-\mu)|\ln \varepsilon|-\mathcal{O}(1) .
$$

The last estimate implies $\mu \geqslant \frac{1-b^{2}}{b^{2} d+1-b^{2}}+o(1)$ which is in contradiction with $\mu \leqslant \frac{1-b^{2}}{2\left(\mathcal{D}_{K, b}+1\right)}$ for $\varepsilon>0$ sufficiently small.

\section{DEFINITION 9.2.}

- For $i \in J_{\mu}$ we let $y_{i} \in \delta \cdot \mathbb{Z}^{2}$ be the unique point s.t. $z_{i} \in B\left(y_{i}, \delta / 2\right)$. Since $\operatorname{dist}\left(z_{i}, \omega_{\varepsilon}\right)<\sqrt{\varepsilon}$ for all $i, y_{i}$ is well defined.

- We denote also $\widetilde{J} \subseteq J_{\mu}$ a set of indices s.t. $\bigcup_{i \in J_{\mu}} B\left(z_{i}, r\right) \subset$ $\bigcup_{k \in \tilde{J}} B\left(y_{k}, 2 \lambda \delta\right)$ and for $k, l \in \widetilde{J}$ s.t. $k \neq l$ we have $y_{k} \neq y_{l}$. We then let for $k \in \widetilde{J}, \widetilde{J}_{k}:=\left\{i \in J_{\mu} \mid z_{i} \in B\left(y_{k}, 2 \lambda \delta\right)\right\}$.

-We may also select "good indices" in order to get well separated centers $y_{k}$ 's. Using Lemma 8.9 with $P=17, \eta=\delta$, there exists a set $\emptyset \neq J^{(y)} \subset J_{\mu}$ and a number $\kappa \in\left\{1,17, \ldots, 17^{\operatorname{Card}\left(J_{\mu}\right)-1}\right\}$ (dependent on $\varepsilon$ ) s.t.

$\left\{\bigcup_{k \in \tilde{J}} B\left(y_{k}, \delta\right) \subset \bigcup_{k \in J^{(y)}} B\left(y_{k}, \kappa \delta\right)\right.$

$\left\{\right.$ for $k, l \in J^{(y)}$ with $k \neq l$ we have $\left|y_{k}-y_{l}\right| \geqslant 16 \kappa \delta$.

We denote, for $k \in J^{(y)}, \widetilde{d}_{k}:=\operatorname{deg}_{\partial B\left(y_{k}, \kappa \delta\right)}(v)$.

- There exists also $\left\{J_{k} \mid k \in J^{(y)}\right\}$, a partition of $J_{\mu}$ with non empty sets (dependent on $\varepsilon$ ), s.t.

$$
B\left(z_{i}, \delta / 2\right) \subset B\left(y_{k}, \kappa \delta\right) \Longleftrightarrow i \in J_{k} \text { for } k \in J^{(y)} \text {. }
$$

We are going to prove that $\widetilde{J}=J_{\mu}$ and for all $k \in J^{(y)}$ we have $J_{k}=\widetilde{J}_{k}$. 
Proposition 9.3. - Assume (9.7), for $\varepsilon>0$ sufficiently small, if $J_{\mu} \neq$ $\emptyset$ then $d_{i}=1$ for all $i \in J_{\mu}$.

Proof. - We argue by contradiction and we assume the existence of a subsequence (still denoted by $\varepsilon=\varepsilon_{n} \downarrow 0$ ) s.t. for all $\varepsilon$ there exists $i_{0} \in J_{\mu}$ s.t. $d_{i_{0}} \geqslant 2$.

From Corollary 8.8(2) applied in $B\left(y_{k}, 2 \lambda \delta\right) \backslash \bigcup_{i \in \tilde{J}_{k}} \overline{B\left(z_{i}, r\right)}$ :

$$
\begin{aligned}
\frac{1}{2} \int_{\Omega_{r}} \alpha|\nabla v|^{2} & \geqslant \sum_{k \in \tilde{J}} \frac{b^{2}}{2} \int_{B\left(y_{k}, 2 \lambda \delta\right) \backslash \bigcup_{i \in \tilde{J}_{k}} \frac{}{B\left(z_{i}, r\right)}|\nabla v|^{2}} \\
& \geqslant \pi b^{2} \sum_{k \in \tilde{J}} \sum_{i \in J_{k}} d_{i}^{2} \ln \left(\frac{\lambda \delta}{r}\right)-\mathcal{O}(1) \\
& \geqslant \pi b^{2}\left(1+\sum_{i \in J_{\mu}} d_{i}\right) \ln \left(\frac{\lambda \delta}{r}\right)-\mathcal{O}(1) .
\end{aligned}
$$

We then get $F(v) \geqslant \pi b^{2}(d|\ln \varepsilon|+|\ln r|)+\mathcal{O}(|\ln (\lambda \delta)|)$. Since $|\ln \varepsilon|=\mathcal{O}(|\ln r|)$ and $|\ln (\lambda \delta)|+\ln h_{\mathrm{ex}}=o(|\ln \varepsilon|)$, this estimate is in contradiction with (9.5) for sufficiently small $\varepsilon$.

Proposition 9.4. - Assume $\mu$ satisfies (9.7) and $J_{\mu} \neq \emptyset$. Then for sufficiently small $\varepsilon>0$ we have $\operatorname{dist}(\mathbf{z}, \Lambda) \leqslant \frac{\ln h_{\mathrm{ex}}}{\sqrt{h_{\mathrm{ex}}}}$.

The proof of the proposition uses the following obvious lemma whose proof is left to the reader.

\section{LEMMA 9.5.}

(1) Let $N \in \mathbb{N}^{*}, \mathbf{D} \in \mathbb{N}^{N}$ and for $k \in\{1, \ldots, N\}$ let $N_{k} \in \mathbb{N}^{*}$ and $\mathbf{D}^{(k)} \in \mathbb{N}^{N_{k}}$ be s.t. $D_{k}=\sum_{i} d_{i}^{(k)}$. Then we have

$$
\sum_{k=1}^{N} D_{k}^{2} \geqslant \sum_{k=1}^{N} \sum_{i=1}^{N_{k}}\left(d_{i}^{(k)}\right)^{2} .
$$

Moreover the equality holds if and only if for all $k \in\{1, \ldots, N\}$ and for all $i \in\left\{1, \ldots, N_{k}\right\}$ we have $d_{i}^{(k)} \in\left\{0, D_{k}\right\}$.

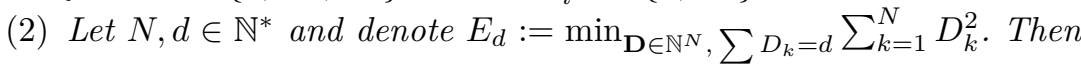
we have for $\mathbf{D} \in \mathbb{N}^{N}$ s.t. $\sum D_{k}=$ d:

$$
\sum_{k=1}^{N} D_{k}^{2}=E_{d} \Longleftrightarrow \mathbf{D} \in\{\lfloor d / N\rfloor ;\lceil d / N\rceil\}^{N} .
$$


Proof of Proposition 9.4. - We argue by contradiction and we assume the existence of a subsequence (still denoted by $\varepsilon=\varepsilon_{n} \downarrow 0$ ) and $i_{0} \in J_{\mu}$ s.t. $\operatorname{dist}\left(z_{i_{0}}, \Lambda\right)>\frac{\ln h_{\mathrm{ex}}}{\sqrt{h_{\mathrm{ex}}}}$.

Then there exists $\eta>0$ (independent of $\varepsilon$ ) s.t.

$$
h_{\mathrm{ex}} \xi_{0}\left(z_{i_{0}}\right) \geqslant-h_{\mathrm{ex}}\left\|\xi_{0}\right\|_{L^{\infty}(\Omega)}+4 \eta\left(\ln h_{\mathrm{ex}}\right)^{2} .
$$

Consequently:

$$
-2 \pi h_{\mathrm{ex}} \sum \xi_{0}\left(z_{i}\right) \leqslant 2 \pi d h_{\mathrm{ex}}\left\|\xi_{0}\right\|_{L^{\infty}(\Omega)}-4 \eta\left(\ln h_{\mathrm{ex}}\right)^{2} .
$$

From $(9.2)$ we get (for small $\varepsilon$ )

$$
\begin{aligned}
F(v) & \leqslant 2 \pi d h_{\mathrm{ex}}\left\|\xi_{0}\right\|_{L^{\infty}(\Omega)}-3 \eta\left(\ln h_{\mathrm{ex}}\right)^{2} \\
(\text { Hyp. }(1.3)) & \leqslant \pi d|\ln \varepsilon|-2 \eta\left(\ln h_{\mathrm{ex}}\right)^{2} .
\end{aligned}
$$

Using (8.6) we get

$$
\frac{1}{2} \int_{\Omega_{r}} \alpha|\nabla v|^{2} \leqslant d \pi\left[b^{2}|\ln r|+\left(1-b^{2}\right)|\ln (\lambda \delta)|\right]-\eta\left(\ln h_{\mathrm{ex}}\right)^{2} .
$$

We let $\chi:=10 \max _{k \in \tilde{J}} \operatorname{dist}\left(y_{k}, \Lambda\right)$ and for $p \in \Lambda, D_{p}:=\operatorname{deg}_{\partial B(p, \chi)}(v)$, $J_{p}:=\left\{k \in J^{(y)} \mid y_{k} \in B(p, \chi)\right\}$. For a latter use we claim that $\chi \geqslant$ $\ln \left(h_{\mathrm{ex}}\right) / \sqrt{h_{\mathrm{ex}}}$ and then

$$
\lambda|\ln \chi| / \chi \rightarrow 0 .
$$

We have (see Definition 9.2 for notation)

$$
\begin{aligned}
& \frac{1}{2} \int_{\Omega_{r}} \alpha|\nabla v|^{2}
\end{aligned}
$$

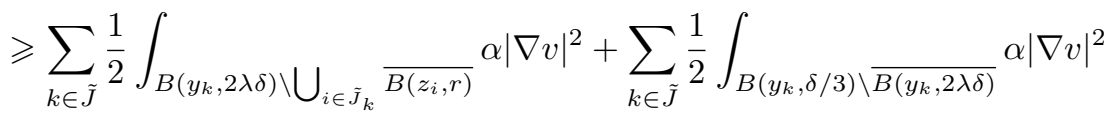

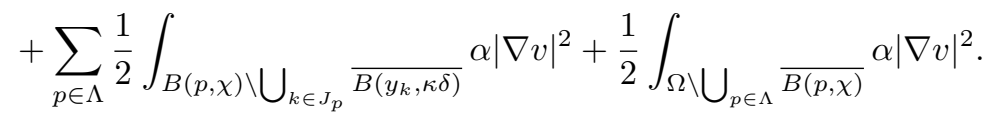

It is clear that, for $k \in \widetilde{J}$, we may use Corollary 8.8(2) in $B\left(y_{k}, 2 \lambda \delta\right) \backslash$ $\bigcup_{i \in \tilde{J}_{k}} \overline{B\left(z_{i}, r\right)}$ in order to get

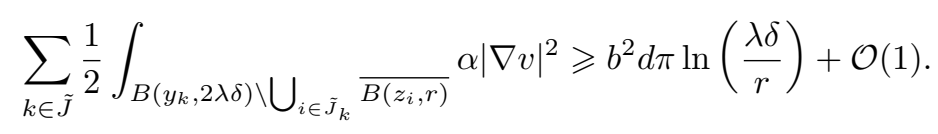

Let $k \in \widetilde{J}$, from (5.8) and Proposition $8.7(2)$ we obtain

$$
\frac{1}{2} \int_{B\left(y_{k}, \delta / 3\right) \backslash \overline{B\left(y_{k}, 2 \lambda \delta\right)}} \alpha|\nabla v|^{2} \geqslant \pi \operatorname{deg}_{\partial B\left(y_{k}, 2 \lambda \delta\right)}(v)^{2}|\ln \lambda|+\mathcal{O}(1) .
$$


Let $p \in \Lambda$ be s.t. $D_{p} \neq 0$, Corollary 8.8(1) gives

$$
\frac{1}{2} \int_{B(p, \chi) \backslash \bigcup_{k \in J_{p}} \frac{}{B\left(y_{k}, \kappa \delta\right)}} \alpha|\nabla v|^{2} \geqslant \pi \sum_{k \in J_{p}} \widetilde{d}_{k}^{2} \ln \left(\frac{\chi}{\delta}\right)+\mathcal{O}(1) .
$$

From Propositions 6.4, 6.5 and 8.10 (with (9.9)) we deduce

$$
\frac{1}{2} \int_{\Omega \backslash \bigcup_{p \in \Lambda} \frac{}{B(p, \chi)}} \alpha|\nabla v|^{2} \geqslant \pi \sum_{p \in \Lambda} D_{p}^{2}|\ln \chi|+\mathcal{O}(1) .
$$

From Lemma 9.5(1) we have

$$
d \leqslant \sum_{k \in \tilde{J}} \operatorname{deg}_{\partial B\left(y_{k}, 2 \lambda \delta\right)}(v)^{2} \leqslant \sum_{p \in \Lambda} \sum_{k \in J_{p}} \widetilde{d}_{k}^{2} \leqslant \sum_{p \in \Lambda} D_{p}^{2} .
$$

Then we get

$$
\frac{1}{2} \int_{\Omega_{r}} \alpha|\nabla v|^{2} \geqslant d \pi\left[b^{2}|\ln r|+\left(1-b^{2}\right)|\ln (\lambda \delta)|\right]+\mathcal{O}(1) .
$$

This estimate is in contradiction with (9.8) for sufficiently small $\varepsilon$.

Proposition 9.6. - Assume $\mu$ satisfies (9.7) and let $\varepsilon=\varepsilon_{n} \downarrow 0$ be a sequence.

(1) If $\operatorname{Card}\left(J_{\mu}\right) \geqslant 2$ then for $\varepsilon>0$ sufficiently small and for $i \neq j$, $\left|z_{i}-z_{j}\right| \geqslant h_{\mathrm{ex}}^{-1} \ln h_{\mathrm{ex}}$.

(2) For $\varepsilon>0$ sufficiently small we have for $p \in \Lambda$, $\operatorname{deg}_{\partial B\left(p, h_{\mathrm{ex}}^{-1 / 2} \ln h_{\mathrm{ex}}\right)}(v) \in$ $\left\{\left\lfloor d / N_{0}\right\rfloor ;\left\lceil d / N_{0}\right\rceil\right\}$.

The proof of Proposition 9.6 is postponed to Appendix I.

Since $\lambda \delta h_{\mathrm{ex}} \rightarrow 0$, Proposition 9.6 implies that each cell of period contains at most a disc $B\left(z_{i}, r\right)$ with $i \in J_{\mu}$.

Following the argument in [6, proof of the third part in Proposition 3.6, see Appendix D-Section 4.5], we may refine Proposition 9.1(2).

Proposition 9.7. - Assume $\mu$ satisfies (9.7), then there is $\eta_{\omega, b}>0$ depending only on $\omega$ and $b$ s.t. for $i \in J_{\mu}$ we have $B\left(z_{i}, 2 \eta_{\omega, b} \lambda \delta\right) \subset \omega_{\varepsilon}$.

Corollary 9.8. - Assume $\mu$ satisfies (9.7). Then we have

$$
\int_{\Omega \backslash \bigcup_{i \in J_{\mu}} B\left(z_{i}, \lambda^{2} \delta^{2}\right)}|\nabla v|^{2}+\frac{1}{\varepsilon^{2}}\left(1-|v|^{2}\right)^{2}=\mathcal{O}(|\ln (\lambda \delta)|) .
$$

Moreover

$$
|v|=1+o(1) \text { in } \Omega \backslash \bigcup_{i \in J_{\mu}} B\left(z_{i}, 2 \lambda^{2} \delta^{2}\right)
$$


Proof. - We have

$\frac{b^{4}}{4} \int_{\Omega \backslash \bigcup_{i \in J_{\mu}} B\left(z_{i}, \lambda^{2} \delta^{2}\right)}|\nabla v|^{2}+\frac{1}{\varepsilon^{2}}\left(1-|v|^{2}\right)^{2} \leqslant F(v)-\sum_{i \in J_{\mu}} F\left(v, B\left(z_{i}, \lambda^{2} \delta^{2}\right)\right)$.

For $i \in J_{\mu}$, from Corollary $8.7(2)$ :

$$
\begin{aligned}
F\left(v, B\left(z_{i}, \lambda^{2} \delta^{2}\right)\right) & \geqslant \frac{b^{2}}{2} \int_{B\left(z_{i}, \lambda^{2} \delta^{2}\right) \backslash \overline{B\left(z_{i}, r\right)}}|\nabla v|^{2}+F\left(v, B\left(z_{i}, r\right)\right) \\
& \geqslant 2 b^{2} \pi \ln (\lambda \delta)+b^{2} \pi|\ln \varepsilon|+\mathcal{O}(1) .
\end{aligned}
$$

Since, by Proposition 9.6, the discs $B\left(z_{i}, \lambda^{2} \delta^{2}\right)$ are pairwise disjoint, we obtain with (9.5):

$$
\frac{b^{4}}{4} \int_{\Omega \backslash \bigcup_{i \in J_{\mu}} B\left(z_{i}, \lambda^{2} \delta^{2}\right)}|\nabla v|^{2}+\frac{1}{\varepsilon^{2}}\left(1-|v|^{2}\right)^{2} \leqslant \mathcal{O}(|\ln (\lambda \delta)|) .
$$

This estimate is equivalent to (9.13).

We are going to prove (9.14). We argue by contradiction and we assume the existence of an extraction still denoted $\varepsilon=\varepsilon_{n} \downarrow 0, t \in(0,1)$ and $\left(x_{n}\right)_{n} \subset$ $\Omega \backslash \bigcup_{i \in J_{\mu}} B\left(z_{i}, 2 \lambda^{2} \delta^{2}\right)$ s.t. $\left|v_{\varepsilon_{n}}\left(x_{n}\right)\right|<t$.

By Proposition 8.1, there exists $C_{t}>0$ s.t. for sufficiently large $n$ :

$$
\int_{B\left(x_{n}, \sqrt{\varepsilon}_{n}\right) \cap \Omega}\left|\nabla v_{\varepsilon_{n}}\right|^{2}+\frac{1}{\varepsilon_{n}^{2}}\left(1-\left|v_{\varepsilon_{n}}\right|^{2}\right)^{2}>C_{t}\left|\ln \varepsilon_{n}\right| .
$$

Moreover, for $n$ sufficiently large to get $\sqrt{\varepsilon_{n}}<\lambda^{2} \delta^{2}$, we have $\left[B\left(x_{n}, \sqrt{\varepsilon_{n}}\right) \cap\right.$ $\Omega] \subset \Omega \backslash \bigcup_{i \in J_{\mu}} B\left(z_{i}, \lambda^{2} \delta^{2}\right)$. This inclusion is in contradiction with (9.13) and (9.15).

From Proposition 9.7, for $i \in J_{\mu}$, we have $\widehat{z}_{i}:=\frac{z_{i}-y_{i}}{\lambda \delta} \in \omega$ where $y_{i} \in \delta \mathbb{Z}^{2}$ is s.t. $z_{i} \in B\left(y_{i}, \lambda \delta\right)$. Moreover, up to considering an extraction, we may assume that, for $i \in J_{\mu}$, there exists $\widehat{z}_{i}^{0} \in \omega$ s.t. $\widehat{z}_{i} \rightarrow \widehat{z}_{i}^{0}$.

We start with the following proposition.

Proposition 9.9. - We have the following sharp lower bound:

$$
\begin{aligned}
\mathcal{F}(v, A) \geqslant h_{\mathrm{ex}}^{2} \mathbf{J}_{\mathbf{0}}+d M_{\Omega}\left[-h_{\mathrm{ex}}+H_{c_{1}}^{0}\right]+\mathscr{L}_{1}(d) \ln h_{\mathrm{ex}}+\mathscr{L}_{2}(d) \\
+\sum_{i \in J_{\mu}}\left[W^{\mathrm{micro}}\left(\widehat{z}_{i}^{0}\right)-\min _{\omega} W^{\mathrm{micro}}\right]+\left[\mathcal{W}_{d}(\mathbf{D})-\overline{\mathcal{W}}_{d}\right]+o(1)
\end{aligned}
$$

where $\overline{\mathcal{W}}_{d}=\min _{\Lambda_{d}} \mathcal{W}_{d}$ is defined in (7.2) and

$$
\mathcal{W}_{d}(\mathbf{D}):=W_{N_{0}}^{\operatorname{macro}}(\mathbf{p}, \mathbf{D})+\sum_{p \in \Lambda} C_{p, D_{p}}+\widetilde{V}\left[\zeta_{(\mathbf{p}, \mathbf{D})}\right]
$$


where for $p \in \Lambda, D \in \mathbb{N}^{*}, C_{p, D}$ is defined in $(6.16), C_{p, 0}:=0$ and $\widetilde{V}\left[\zeta_{(\mathbf{p}, \mathbf{D})}\right]$ is defined in Proposition 5.13.

We split the proof of Proposition 9.9 in several lemmas.

The first step is the following lemma consisting in a "macroscopic/ mesoscopic" version of Proposition 9.9.

LEMMA 9.10. - Let $\rho=|v|$ and $w=v / \rho$ in $\Omega \backslash \bigcup_{i \in J_{\mu}} \overline{B\left(y_{i}, \delta / 3\right)}$. We then have

$$
\begin{aligned}
& \frac{1}{2} \int_{\Omega \backslash \bigcup_{i \in J_{\mu}}} \frac{\rho^{2}\left(y_{i}, \delta / 3\right)}{\rho \rho^{2}|\nabla w|^{2}} \\
& \geqslant d \pi|\ln (\delta / 3)|-\pi \sum_{\substack{p \in \Lambda \\
b b D_{p} \geqslant 2}} \sum_{\substack{i, j \in J_{p} \\
i \neq j}} \ln \left|z_{i}-z_{j}\right|+W_{N_{0}}^{\operatorname{macro}}(\mathbf{p}, \mathbf{D})+o(1) .
\end{aligned}
$$

Proof. - On the one hand, from Proposition 9.4 and letting $\chi:=h_{\mathrm{ex}}^{-1 / 4}$ we have $|v| \geqslant 1 / 2$ in $\Omega \backslash \bigcup_{p \in \Lambda} \overline{B(p, \chi)}$. Then, from Proposition 8.10, we have

$$
\frac{1}{2} \int_{\Omega \backslash \bigcup_{p \in \Lambda} \frac{}{B(p, \chi)}} \alpha|\nabla v|^{2} \geqslant \pi \sum_{p \in \Lambda} D_{p}^{2}|\ln \chi|+W_{N_{0}}^{\text {macro }}(\mathbf{p}, \mathbf{D})+o(1) .
$$

On the other hand, from Proposition 9.6, if $\operatorname{Card}\left(J_{\mu}\right) \geqslant 2$ then, for $i, j \in J_{\mu}$ with $i \neq j$, we have $\left|y_{i}-y_{j}\right| \geqslant h_{\mathrm{ex}}^{-1} \ln \left(h_{\mathrm{ex}}\right)-2 \lambda \delta$.

Consequently, if $D_{p}=\operatorname{deg}_{\partial B\left(p, \eta_{\Omega}\right)}(v) \neq 0\left(\eta_{\Omega}\right.$ is defined in (6.14)), letting $J_{p}:=\left\{i \in J_{\mu} \mid z_{i} \in B\left(p, \eta_{\Omega}\right)\right\}, \mathcal{D}_{p}:=B(p, \chi) \backslash \bigcup_{i \in J_{p}} \overline{B\left(y_{i}, h_{\mathrm{ex}}^{-1}\right)}$,

$$
\begin{aligned}
\Phi: B(p, \chi) & \longrightarrow \mathbb{D}=B(0,1) \\
x & \longmapsto \frac{x-p}{\chi},
\end{aligned}
$$

$\widehat{v}=v \circ \Phi^{-1}, \widehat{\alpha}=\alpha \circ \Phi^{-1}, \widehat{\mathcal{D}}_{p}:=\Phi\left(\mathcal{D}_{p}\right)$ and $\widehat{y}_{i}:=\Phi\left(y_{i}\right)$ for $y_{i} \in B(p, \chi)$, then we may apply Proposition 8.10. Writing $\left(\widehat{\mathbf{y}}_{p}, \mathbb{1}\right):=\left\{\left(\widehat{y}_{i}, 1\right) \mid i \in J_{p}\right\}$, Proposition 8.10 gives:

$$
\frac{1}{2} \int_{\mathcal{D}_{p}} \alpha|\nabla v|^{2}=\frac{1}{2} \int_{\hat{\mathcal{D}}_{p}} \widehat{\alpha}|\nabla \widehat{v}|^{2} \geqslant \pi D_{p} \ln \left(\chi h_{\mathrm{ex}}\right)+W_{D_{p}, \mathbb{D}}^{\operatorname{macro}}\left(\widehat{\mathbf{y}}_{p}, \mathbb{1}\right)+o(1)
$$

where $W_{D_{p}, \mathbb{D}}^{\text {macro }}$ is the macroscopic renormalized energy in the unit disc $\mathbb{D}$ with $D_{p}$ points.

From Proposition 1 in [13] we have

$$
W_{D_{p}, \mathbb{D}}^{\operatorname{macro}}\left(\widehat{\mathbf{y}}_{p}, \mathbb{1}\right)=-\pi \sum_{\substack{i, j \in J_{p} \\ i \neq j}}\left[\ln \left|\widehat{y}_{i}-\widehat{y}_{j}\right|-\ln \left|1-\widehat{y}_{i} \widehat{\widehat{y}}_{j}\right|\right]+\pi \sum_{i \in J_{p}} \ln \left(1-\left|\widehat{y}_{i}\right|^{2}\right) \text {. }
$$


Using Proposition 9.4, we get for $i \in J_{p},\left|\widehat{y}_{i}\right| \leqslant \frac{h_{\mathrm{ex}}^{-1 / 2} \ln h_{\mathrm{ex}}}{\chi}=o(1)$ and then

$$
W_{D_{p}, \mathbb{D}}^{\mathrm{macro}}\left(\widehat{\mathbf{y}}_{p}, \mathbb{1}\right)=-\pi \sum_{\substack{i, j \in J_{p} \\ i \neq j}} \ln \left|y_{i}-y_{j}\right|-\pi\left(D_{p}^{2}-D_{p}\right)|\ln \chi|+o(1) .
$$

For $i \in J_{\mu}$, we let $\mathscr{R}_{i}:=B\left(y_{i}, h_{\text {ex }}^{-1}\right) \backslash \overline{B\left(y_{i}, \delta / 3\right)}$. With Proposition 8.7(1) we obtain

$$
\frac{1}{2} \int_{\mathscr{R}_{i}} \alpha|\nabla v|^{2} \geqslant \pi\left|\ln \left(\delta h_{\mathrm{ex}} / 3\right)\right| .
$$

By combining (9.17), (9.18), (9.19) and (9.20) the result is proved.

The second step is a "microscopic" version of Proposition 9.9.

LEMMA 9.11. - If $r \leqslant \widetilde{r} \leqslant \lambda^{2} \delta^{2}$, then:

$$
\sum_{i \in J_{\mu}} F\left(v, \mathscr{R}_{i}\right) \geqslant d \pi\left(|\ln (3 \lambda)|+b^{2}|\ln (\lambda \delta / \widetilde{r})|\right)+\sum_{i \in J_{\mu}} W^{\text {micro }}\left(\widehat{z}_{i}^{0}\right)+o(1)
$$

where, for $i \in J_{\mu}, \mathscr{R}_{i}:=B\left(y_{i}, \delta / 3\right) \backslash \overline{B\left(z_{i}, \widetilde{r}\right)}$.

Proof. - Note that in order to prove Lemma 9.11 (up to replacing $v$ by $\underline{v}$ ) we may assume $\rho=|v| \leqslant 1$.

We first consider the case $\lambda^{4} \delta^{4} \leqslant \widetilde{r} \leqslant \lambda^{2} \delta^{2}$. We then may assume

$$
\sum_{i \in J_{\mu}} F_{\varepsilon}\left(v, \mathscr{R}_{i}\right)=\mathcal{O}(|\ln (\lambda \delta)|)
$$

since in the contrary case there is nothing to prove.

Fix $i \in J_{\mu}$ and let $v_{\star}$ be a minimizer of $F_{\varepsilon}\left(\cdot, \mathscr{R}_{i}\right)$ in $H^{1}\left(\mathscr{R}_{i}, \mathbb{C}\right)$ with the Dirichlet boundary condition $\operatorname{tr}_{\partial \mathscr{R}_{i}}(\cdot)=\operatorname{tr}_{\partial \mathscr{R}_{i}}(v)$. Note that such minimizers exist and we have $F_{\varepsilon}\left(v_{\star}, \mathscr{R}_{i}\right) \leqslant F_{\varepsilon}\left(v, \mathscr{R}_{i}\right)=\mathcal{O}(|\ln (\lambda \delta)|)$.

The key ingredient consists in noting that since $v_{\star}$ is a minimizer of a weighted Ginzburg-Landau type energy we may thus use a sharp interior $\eta$-ellipticity result. Namely, following the strategy of [9] to prove Lemma 1 (see [9, Appendix C]), by using the first part of the proof (the interior argument which does not required any information on $\left.\operatorname{tr}_{\partial \mathscr{R}_{i}}\left(v_{\star}\right)\right)$, we get

$$
\begin{aligned}
\rho_{\star}:=\left|v_{\star}\right| \geqslant 1-\mathcal{O}(\sqrt{|\ln (\lambda \delta)| /|\ln \varepsilon|}) & \\
& \text { in } \widetilde{\mathscr{R}}_{i}:=B\left(y_{i}, \delta / 3-\varepsilon^{1 / 4}\right) \backslash \overline{B\left(z_{i}, \widetilde{r}+\varepsilon^{1 / 4}\right) .}
\end{aligned}
$$

Write in $\widetilde{\mathscr{R}}_{i}: v_{\star}=\rho_{\star} w_{\star}$ where $w_{\star} \in H^{1}\left(\widetilde{\mathscr{R}}, \mathbb{S}^{1}\right)$.

Note that by (1.2) (namely $|\ln (\lambda \delta)|=\mathcal{O}(\ln |\ln \varepsilon|))$ we have $|\ln (\lambda \delta)|^{3} /|\ln \varepsilon|=$ $o(1)$ and then from (9.21) and (9.22) (and also $\rho_{\star} \leqslant 1$ ) we have

$$
\int_{\tilde{\mathscr{R}}_{i}} \alpha \rho_{\star}^{2}\left|\nabla w_{\star}\right|^{2}=\int_{\tilde{\mathscr{R}}_{i}} \alpha\left|\nabla w_{\star}\right|^{2}+o(1) .
$$


We then immediately get:

$$
\begin{aligned}
F\left(v, \mathscr{R}_{i}\right) \geqslant F\left(v_{\star}, \mathscr{R}_{i}\right) & \geqslant \frac{1}{2} \int_{\tilde{\mathscr{R}}_{i}} \alpha\left|\nabla w_{\star}\right|^{2}+o(1) \\
& \geqslant \inf _{\substack{\tilde{w} \in H^{1}\left(\tilde{\mathscr{R}}_{i}, \mathbb{S}^{1}\right) \\
\operatorname{deg}(\tilde{w})=1}} \frac{1}{2} \int_{\tilde{\mathscr{R}}_{i}} \alpha|\nabla \widetilde{w}|^{2}+o(1) .
\end{aligned}
$$

It suffices now to claim that from (6.21) we have

$$
\inf _{\substack{\tilde{w} \in H^{1}\left(\tilde{\mathscr{R}}_{i}, \mathbb{S}^{1}\right) \\ \operatorname{deg}(\tilde{w})=1}} \frac{1}{2} \int_{\tilde{\mathscr{R}}_{i}} \alpha|\nabla \widetilde{w}|^{2}=\pi\left(|\ln (3 \lambda)|+b^{2}|\ln (\lambda \delta / \widetilde{r})|\right)+W^{\text {micro }}\left(\widehat{z}_{i}^{0}\right)+o(1)
$$

in order to get $F\left(v, \mathscr{R}_{i}\right) \geqslant \pi\left(|\ln (3 \lambda)|+b^{2}|\ln (\lambda \delta / \widetilde{r})|\right)+W^{\text {micro }}\left(\widehat{z}_{i}^{0}\right)+o(1)$. By summing these lower bounds we get the result.

We complete the proof by considering the cases $r \leqslant \widetilde{r}<\lambda^{4} \delta^{4}$. With Proposition 8.7.2 we get

$$
\begin{aligned}
\frac{1}{2} \int_{B\left(z_{i}, \lambda^{4} \delta^{4}\right) \backslash \frac{\left.z_{i}, \tilde{r}\right)}{B\left(z^{2}\right.}} \alpha|\nabla v|^{2} & \geqslant \frac{b^{2}}{2} \int_{B\left(z_{i}, \lambda^{4} \delta^{4}\right) \backslash \frac{\lambda_{B\left(z_{i}, \tilde{r}\right)}}{}|\nabla v|^{2}} \\
& \geqslant \pi b^{2} \ln \frac{\lambda^{4} \delta^{4}}{\widetilde{r}}-o(1) .
\end{aligned}
$$

This estimate ends the proof.

LEMMA 9.12. - There exists $r \leqslant \widetilde{r}=o\left(\lambda^{2} \delta^{2}\right)$ s.t. for $i \in J_{\mu}$ we have

$$
F\left[v, B\left(z_{i}, \widetilde{r}\right)\right] \geqslant b^{2}[\pi \ln (\widetilde{r} / \varepsilon)+\ln b+\gamma]+o(1) .
$$

Proof. - We first note that we have

$$
\begin{aligned}
\sum_{i \in J_{\mu}} F\left[v, B\left(z_{i}, \lambda^{2} \delta^{2}\right) \backslash \overline{B\left(z_{i}, r\right)}\right] & \\
& \leqslant d b^{2} \pi \ln \left(\lambda^{2} \delta^{2} / r\right)+\mathscr{L}_{1}(d) \ln h_{\mathrm{ex}}+\mathcal{O}(1) .
\end{aligned}
$$

The above estimate is proved by contradiction and assuming the existences of an extraction (still denoted by $\varepsilon=\varepsilon_{n} \downarrow 0$ ) and of a sequence $R_{n} \uparrow \infty$ s.t.

$$
\sum_{i \in J_{\mu}} F\left[v, B\left(z_{i}, \lambda^{2} \delta^{2}\right) \backslash \overline{B\left(z_{i}, r\right)}\right] \geqslant d b^{2} \pi \ln \left(\lambda^{2} \delta^{2} / r\right)+\mathscr{L}_{1}(d) \ln h_{\mathrm{ex}}+R_{n} .
$$

From (8.7) we get

$$
\sum_{i \in J_{\mu}} F\left[v, B\left(z_{i}, \lambda^{2} \delta^{2}\right)\right] \geqslant d b^{2} \pi \ln \left(\lambda^{2} \delta^{2} / \varepsilon\right)+\mathscr{L}_{1}(d) \ln h_{\mathrm{ex}}+R_{n}+\mathcal{O}(1) .
$$

Using Lemmas 9.10 and 9.11 we get an estimate which contradicts (9.5). 
By a classical argument, for sufficiently small $\varepsilon$, there exists $\sqrt{r} \leqslant \widetilde{r} \leqslant$ $r^{1 / 4}$ s.t. for $i \in J_{\mu}$

$$
\frac{\widetilde{r}}{2} \int_{\partial B\left(z_{i}, \tilde{r}\right)}|\nabla v|^{2}+\frac{b^{2}}{2 \varepsilon^{2}}\left(1-|v|^{2}\right)^{2} \leqslant \pi+\frac{4 \mathscr{L}_{1}(d) \ln h_{\mathrm{ex}}+\mathcal{O}(1)}{|\ln r|}
$$

Arguing as in the proof of Proposition 8.3 (Step 3 in Appendix G) it is clear that we may assume $|v| \geqslant 1-|\ln \varepsilon|^{-2}$ on $\partial B\left(z_{i}, \widetilde{r}\right)$ for $i \in J_{\mu}$.

We now define for $i \in J_{\mu}, \rho_{i}:=\operatorname{tr}_{\partial B\left(z_{i}, \tilde{r}\right)}(|v|), w_{i}:=\operatorname{tr}_{\partial B\left(z_{i}, \tilde{r}\right)}(v /|v|)$. We immediately get

$$
\frac{\tilde{r}}{2} \int_{\partial B\left(z_{i}, \tilde{r}\right)}\left|\nabla w_{i}\right|^{2}=\pi+o(1), \quad \frac{\tilde{r}}{2} \int_{\partial B\left(z_{i}, \tilde{r}\right)}\left|\nabla \rho_{i}\right|^{2}+\frac{b^{2}}{2 \varepsilon^{2}}\left(1-\rho_{i}^{2}\right)^{2}=o(1) .
$$

On the other hand, since $\operatorname{deg}\left(w_{i}\right)=1$, there exists $\phi_{i}=\phi_{i, \varepsilon} \in H^{1}((0,2 \pi), \mathbb{R})$ s.t. $\phi_{i}(0)=\phi_{i}(2 \pi) \in[0,2 \pi)$ and $w_{i}\left(z_{i}+\widetilde{r} \mathrm{e}^{\imath \theta}\right)=\mathrm{e}^{-\imath\left(\theta+\phi_{i}(\theta)\right)}$. A direct calculation gives:

$$
2 \pi+o(1)=\widetilde{r} \int_{\partial B\left(z_{i}, \tilde{r}\right)}\left|\partial_{\tau} w_{i}\right|^{2}=\int_{0}^{2 \pi}\left|\left(\phi_{i}+\theta\right)^{\prime}\right|^{2}=2 \pi+\int_{0}^{2 \pi}\left|\phi_{i}^{\prime}\right|^{2} .
$$

The last equalities imply $\phi_{i}^{\prime} \rightarrow 0$ in $L^{2}(0,2 \pi)$ and then $\phi_{i}-\phi_{i}(0) \rightarrow 0$ in $L^{2}(0,2 \pi)$. Hence, up to a subsequence, we get the existence of $\theta_{i} \in[0,2 \pi]$ s.t. $\phi_{i} \rightarrow \theta_{i}$ in $H^{1}(0,2 \pi)$.

We now define $\widetilde{w}_{i} \in H^{1}\left(B\left(z_{i}, 2 \widetilde{r}\right) \backslash \overline{B\left(z_{i}, \widetilde{r}\right)}, \mathbb{S}^{1}\right)$ by $\widetilde{w}_{i}\left(z_{i}+s \mathrm{e}^{\imath \theta}\right)=\mathrm{e}^{\imath\left[\theta+\tilde{\phi}_{i}\left(z_{i}+s \mathrm{e}^{\imath \theta}\right)\right]}$ with $\widetilde{\phi}_{i}\left(z_{i}+s \mathrm{e}^{\imath \theta}\right)=\left[\phi_{i}(\theta)-\theta_{i}\right] \frac{2 \widetilde{r}-s}{\widetilde{r}}+\theta_{i}$.

A direct calculation gives $\int_{B\left(z_{i}, 2 \tilde{r}\right) \backslash \overline{B\left(z_{i}, \tilde{r}\right)}}\left|\nabla \widetilde{\phi}_{i}\right|^{2}=o(1)$ and then

$$
\begin{aligned}
\frac{1}{2} \int_{B\left(z_{i}, 2 \tilde{r}\right) \backslash \overline{B\left(z_{i}, \tilde{r}\right)}}\left|\nabla \widetilde{w}_{i}\right|^{2} & =\frac{1}{2} \int_{B\left(z_{i}, 2 \tilde{r}\right) \backslash \overline{B\left(z_{i}, \tilde{r}\right)}}\left|\nabla\left[\theta+\widetilde{\phi}_{i}\left(z_{i}+s \mathrm{e}^{\imath \theta}\right)\right]\right|^{2}+o(1) \\
& =\pi \ln (2)+o(1) .
\end{aligned}
$$

Let $\widetilde{\rho}_{i} \in H^{1}\left[B\left(z_{i}, 2 \widetilde{r}\right) \backslash \overline{B\left(z_{i}, \widetilde{r}\right)}, \mathbb{R}^{+}\right]$be s.t. $\widetilde{\rho}_{i}\left(z_{i}+s \mathrm{e}^{\imath \theta}\right):=\widetilde{\rho}_{i}\left(z_{i}+\widetilde{r} \mathrm{e}^{\imath \theta}\right) \frac{2 \tilde{r}-s}{\tilde{r}}+$ $\frac{s-\tilde{r}}{\tilde{r}}$.

We then have $F\left[\widetilde{\rho}_{i}, B\left(z_{i}, 2 \widetilde{r}\right) \backslash \overline{B\left(z_{i}, \widetilde{r}\right)}\right]=o(1)$. Consequently, letting $v_{i}:=$ $\widetilde{\rho}_{i} \widetilde{w}_{i} \in H^{1}\left[B\left(z_{i}, 2 \widetilde{r}\right) \backslash \overline{B\left(z_{i}, \widetilde{r}\right)}, \mathbb{C}\right]$ we have

$$
F\left[v_{i}, B\left(z_{i}, 2 \widetilde{r}\right) \backslash \overline{B\left(z_{i}, \widetilde{r}\right)}\right]=\frac{b^{2}}{2} \int_{B\left(z_{i}, 2 \tilde{r}\right) \backslash \overline{B\left(z_{i}, \tilde{r}\right)}}\left|\nabla \widetilde{w}_{i}\right|^{2}+o(1) .
$$

In order to conclude we let

$$
u_{i}:= \begin{cases}v_{i} & \text { in } B\left(z_{i}, 2 \widetilde{r}\right) \backslash \overline{B\left(z_{i}, \widetilde{r}\right)} \\ v & \text { in } B\left(z_{i}, \widetilde{r}\right)\end{cases}
$$


It is clear that $u_{i}\left(z_{i}+2 \widetilde{r} \mathrm{e}^{\imath \theta}\right)=\mathrm{e}^{\imath \theta_{i}} \mathrm{e}^{\imath \theta}$ and then, using Lemma IX.1 in [4], we get

$$
F\left[u_{i}, B\left(z_{i}, 2 \widetilde{r}\right)\right] \geqslant b^{2}[\pi \ln (2 \widetilde{r} / \varepsilon)+\gamma+\pi \ln b]+o(1) .
$$

The last estimate ends the proof of the lemma.

Proof of Proposition 9.9. - From the three previous lemmas we have

$$
\begin{aligned}
F(v) \geqslant & d \pi\left[b^{2}|\ln \varepsilon|+\left(1-b^{2}\right)|\ln (\lambda \delta)|\right]-\pi \sum_{\substack{p \in \Lambda \\
b b D_{p} \geqslant 2}} \sum_{\substack{i, j \in J_{p} \\
i \neq j}} \ln \left|z_{i}-z_{j}\right| \\
& +W_{N_{0}}^{\text {macro }}(\mathbf{p}, \mathbf{D})+\sum_{i \in J_{\mu}} W^{\text {micro }}\left(\widehat{z}_{i}^{0}\right)+d b^{2}[\pi \ln b+\gamma]+o(1) .
\end{aligned}
$$

On the other hand, with Corollary 5.20 (estimate (5.30)) we get

$$
\mathcal{F}(v, A) \geqslant h_{\mathrm{ex}}^{2} \mathbf{J}_{\mathbf{0}}+2 \pi h_{\mathrm{ex}} \sum_{i \in J_{\mu}} \xi_{0}\left(z_{i}\right)+F(v)+\widetilde{V}\left[\zeta_{(\mathbf{p}, \mathbf{D})}\right]+o(1)
$$

where $\zeta_{(\mathbf{p}, \mathbf{D})}$ is defined in Proposition 5.12.

From Proposition 6.10 (estimate (6.15)), for $p \in \Lambda$ s.t. $D_{p} \geqslant 2$, we have:

$$
\begin{aligned}
-\pi \sum_{\substack{i, j \in J_{p} \\
i \neq j}} \ln \left|z_{i}-z_{j}\right|+2 \pi h_{\mathrm{ex}} \sum_{i}\left[\xi_{0}\left(z_{i}\right)-\xi_{0}(p)\right] \\
\geqslant \frac{\pi}{2}\left(D_{p}^{2}-D_{p}\right) \ln \left(\frac{h_{\mathrm{ex}}}{D_{p}}\right)+C_{p, D_{p}}+o(1) .
\end{aligned}
$$

By combining (9.24), (9.25) and (9.26) (and also $\left.\xi_{0} \leqslant 0\right)$ we obtain

$$
\begin{aligned}
\mathcal{F}(v, A) \geqslant & h_{\mathrm{ex}}^{2} \mathbf{J}_{\mathbf{0}}+d \pi\left[b^{2}|\ln \varepsilon|+\left(1-b^{2}\right)|\ln (\lambda \delta)|\right]-2 \pi d h_{\mathrm{ex}}\left\|\xi_{0}\right\|_{L^{\infty}(\Omega)} \\
& +\frac{\pi}{2} \sum_{\substack{p \in \Lambda \\
b b D_{p} \geqslant 2}}\left[\left(D_{p}^{2}-D_{p}\right) \ln \left(\frac{h_{\mathrm{ex}}}{D_{p}}\right)+C_{p, D_{p}}\right]+W_{N_{0}}^{\operatorname{macro}}(\mathbf{p}, \mathbf{D}) \\
& +\sum_{i \in J_{\mu}} W^{\text {micro }}\left(\widehat{z}_{i}^{0}\right)+\widetilde{V}\left[\zeta_{(\mathbf{p}, \mathbf{D})}\right]+d b^{2}[\pi \ln b+\gamma]+o(1)
\end{aligned}
$$

It suffices to see that, since $\mathbf{D} \in \Lambda_{d}$, from the definition of $\mathscr{L}_{1}(d)$ we have

$$
\frac{\pi}{2} \sum_{\substack{p \in \Lambda \\ b b D_{p} \geqslant 2}}\left(D_{p}^{2}-D_{p}\right) \ln \left(\frac{h_{\mathrm{ex}}}{D_{p}}\right)=\mathscr{L}_{1}(d) \ln h_{\mathrm{ex}}+\frac{\pi}{2} \sum_{p \in \Lambda}\left(D_{p}-D_{p}^{2}\right) \ln \left(D_{p}\right)
$$


in order to deduce from (9.27) that

$$
\begin{aligned}
\mathcal{F}(v, A) \geqslant & h_{\mathrm{ex}}^{2} \mathbf{J}_{\mathbf{0}}+d \pi\left[-2 h_{\mathrm{ex}}\left\|\xi_{0}\right\|_{L^{\infty}(\Omega)}+b^{2}|\ln \varepsilon|+\left(1-b^{2}\right)|\ln (\lambda \delta)|\right] \\
& +\mathscr{L}_{1}(d) \ln h_{\mathrm{ex}}+\sum_{i \in J_{\mu}} W^{\mathrm{micro}}\left(\widehat{z}_{i}^{0}\right)+\mathcal{W}_{d}(\mathbf{D}) \\
& +\frac{\pi}{2} \sum_{p \in \Lambda}\left(D_{p}-D_{p}^{2}\right) \ln \left(D_{p}\right)+d b^{2}[\pi \ln b+\gamma]+o(1)
\end{aligned}
$$

where $\mathcal{W}_{d}(\mathbf{D})$ is defined in (9.16). This estimate with the definition of $H_{c_{1}}^{0}$ and $\overline{\mathcal{W}}_{d}$ (see (7.2), (7.5) and (7.6)) ends the proof of the proposition.

\section{The first critical field and the location of the vorticity defects}

We assume that $\lambda, \delta, h_{\mathrm{ex}}$ satisfy (1.2) and (1.3) for some $K \geqslant 0$ independent of $\varepsilon$. We assume also (1.4). We consider a sequence $\varepsilon=\varepsilon_{n} \downarrow 0$.

As in the previous section we focus on sequences of quasi-minimizers of $\mathcal{F}$. For simplicity we write $(v, A)$ instead of $\left(v_{\varepsilon}, A_{\varepsilon}\right)$. We assume that $(3.11)$ and (8.1) holds and since (3.11) and (8.1) are gauge invariant we may also assume that $(v, A)$ is in the Coulomb gauge.

From above results, for a fixed $\mu>0$ sufficiently small (satisfying (9.7)) and for $\varepsilon>0$ sufficiently small, there exists a (finite) set $\mathcal{Z} \subset \Omega$, depending on $\varepsilon$ and possibly empty s.t. letting $d:=\operatorname{Card}(\mathcal{Z})$ (we write $\mathcal{Z}=$ $\left.\left\{z_{1}, \ldots, z_{2}\right\}\right)$ :

- If $d=0$, then $|v| \geqslant 1 / 2$ in $\Omega$.

- If $d>0$, then $\left|z_{i}-z_{j}\right| \gtrsim h_{\mathrm{ex}}^{-1} \ln h_{\mathrm{ex}}$ if $i \neq j,|v| \geqslant 1 / 2$ in $\Omega \backslash$ $\bigcup_{i=1}^{d} \overline{B\left(z_{i}, \varepsilon^{\mu}\right)}$ and $\operatorname{deg}_{\partial B\left(z, \varepsilon^{\mu}\right)}(v)=1$ for $z \in \mathcal{Z}$.

Moreover $d=\mathcal{O}(1)$. Then if needed, up to a subsequence, we may assume that $d$ is independent of $\varepsilon$.

By combining Corollary 5.10, Propositions 6.10, 7.3, 9.6 and 9.9 we get the following corollary.

Corollary 10.1. - Assume $\lambda, \delta, h_{\mathrm{ex}}$ satisfy (1.2) and (1.3) for some $K \geqslant 0$ independent of $\varepsilon$. Let $\varepsilon=\varepsilon_{n} \downarrow 0$ and let $\left(\left(v_{\varepsilon}, A_{\varepsilon}\right)\right)_{\varepsilon} \subset \mathscr{H}$ be a sequence satisfying (3.11) and (8.1). Assume that $d$ is independent of $\varepsilon$. Without loss of generality we may assume that $\left(v_{\varepsilon}, A_{\varepsilon}\right)$ is in the Coulomb gauge. We have $\mathcal{F}\left(v_{\varepsilon}, A_{\varepsilon}\right)=h_{\mathrm{ex}}^{2} \mathbf{J}_{0}+d M_{\Omega}\left[-h_{\mathrm{ex}}+H_{c_{1}}^{0}\right]+\mathscr{L}_{1}(d) \ln h_{\mathrm{ex}}+\mathscr{L}_{2}(d)+o(1) .(10.1)$

Moreover, if $d \neq 0$ then: 
- We have $\mathbf{D} \in \Lambda_{d}$ (see (7.1)) and $\mathbf{D}$ minimises $\mathcal{W}_{d}$ in $\Lambda_{d}$ where $\mathcal{W}_{d}$ is defined in (9.16).

- For $p \in \Lambda$ s.t. $D_{p}>0$ and $i \in J_{p}$, we denote $\breve{z}_{i}:=\left(z_{i}-p\right) \sqrt{D_{p} / h_{\mathrm{ex}}}$ and $\breve{\mathbf{z}}_{p}:=\left\{\breve{z}_{i} \mid i \in J_{p}\right\}$. Then, up to a subsequence, $\breve{\mathbf{z}}_{p}$ converges to a minimizer of $W_{p, D_{p}}^{\text {meso }}$ defined in (6.17).

- For $i \in\{1, \ldots, d\}$, we write $\widehat{z}_{i}:=\left(z_{i}-y_{i}\right) /(\lambda \delta) \in \omega$ where $y_{i} \in \delta \mathbb{Z}^{2}$ is s.t. $z_{i} \in B\left(y_{i}, \lambda \delta\right)$. Then, up to a subsequence, $\widehat{z}_{i}$ converges to a minimizer of $W^{\text {micro }}$.

For a further use, we claim that for $d_{0} \geqslant 0$, from Proposition 7.3, there exists a configuration $\left(v^{0}, A^{0}\right) \in \mathscr{H}$ which is in the Coulomb gauge s.t.

$$
\begin{aligned}
\mathcal{F}\left(v^{0}, A^{0}\right) & -h_{\mathrm{ex}}^{2} \mathbf{J}_{\mathbf{0}} \\
& =d_{0} M_{\Omega}\left[-h_{\mathrm{ex}}+H_{c_{1}}^{0}\right]+\mathscr{L}_{1}\left(d_{0}\right) \ln h_{\mathrm{ex}}+\mathscr{L}_{2}\left(d_{0}\right)+o(1) .
\end{aligned}
$$

Recall that, from Lemma 7.1, for $d \neq 0$, we have $d \in\left\{1, \ldots, N_{0}\right\}$ if and only if $\mathscr{L}_{1}(d)=0$ and $\mathscr{L}_{2}(d)=\overline{\mathcal{W}}_{d}$.

For further use, in the next lemma, we define four kinds of numbers $\Delta_{d}^{(1)}$, $\Delta_{d^{\prime}, d}^{(1)}, \Delta_{d}^{(2)}$ and $\Delta_{d^{\prime}, d}^{(2)}$. Lemma 10.2 (whose proof is left to the reader) is dedicated to give some explicit expressions of this numbers based on basic computations.

LEMMA 10.2. - For $0 \leqslant d<d^{\prime}$ we let:

(1) $\Delta_{d}^{(1)}:=\frac{\mathscr{L}_{1}(d+1)-\mathscr{L}_{1}(d)}{M_{\Omega}}=\frac{\pi}{M_{\Omega}}\left\lfloor\frac{d}{N_{0}}\right\rfloor$.

(2) $\Delta_{d^{\prime}, d}^{(1)}:=\frac{\mathscr{L}_{1}\left(d^{\prime}\right)-\mathscr{L}_{1}(d)}{M_{\Omega}\left(d^{\prime}-d\right)}=\frac{\pi}{M_{\Omega}\left(d^{\prime}-d\right)} \sum_{k=d}^{d^{\prime}-1}\left\lfloor\frac{k}{N_{0}}\right\rfloor$.

(3) $\Delta_{d}^{(2)}:=\frac{\mathscr{L}_{2}(d+1)-\mathscr{L}_{2}(d)}{M_{\Omega}}$ and

$\Delta_{d}^{(2)}-\frac{\overline{\mathcal{W}}_{d+1}-\overline{\mathcal{W}}_{d}}{M_{\Omega}}$

$= \begin{cases}0 & \text { if } d \leqslant N_{0}-1, \\ -\frac{\pi}{2 M_{\Omega}}\left\lfloor\frac{d}{N_{0}}\right\rfloor\left[\left(1+\left\lfloor\frac{d}{N_{0}}\right\rfloor\right) \ln \left(1+\left\lfloor\frac{d}{N_{0}}\right\rfloor\right)+\left(1-\left\lfloor\frac{d}{N_{0}}\right\rfloor\right) \ln \left\lfloor\frac{d}{N_{0}}\right\rfloor\right] & \text { if } d \geqslant N_{0} .\end{cases}$

(4) $\Delta_{d^{\prime}, d}^{(2)}:=\frac{\mathscr{L}_{2}\left(d^{\prime}\right)-\mathscr{L}_{2}(d)}{M_{\Omega}\left(d^{\prime}-d\right)}$ thus, if $d^{\prime} \leqslant N_{0}$, then $\Delta_{d^{\prime}, d}^{(2)}=\frac{\overline{\mathcal{W}}_{d^{\prime}}-\overline{\mathcal{W}}_{d}}{M_{\Omega}\left(d^{\prime}-d\right)}$.

By using (10.1) and (10.2) we easily get the following corollary.

Corollary 10.3. - Let $\varepsilon=\varepsilon_{n} \downarrow 0, \lambda, \delta, h_{\mathrm{ex}}$ and $\left(\left(v_{\varepsilon}, A_{\varepsilon}\right)\right)_{\varepsilon} \subset \mathscr{H}$ be as in Corollary 10.1.

Assume that $d$ is independent of $\varepsilon$. Then we have for $d^{\prime}>d$

$$
h_{\mathrm{ex}} \leqslant H_{c_{1}}^{0}+\Delta_{d^{\prime}, d}^{(1)} \times \ln h_{\mathrm{ex}}+\Delta_{d^{\prime}, d}^{(2)}+o(1) .
$$


Then, letting $\chi$ be s.t. $h_{\mathrm{ex}}=H_{c_{1}}^{0}(1+\chi)(\chi=o(1)$ from $(1.3))$, we have thus

$$
h_{\mathrm{ex}} \leqslant H_{c_{1}}^{0}+\Delta_{d^{\prime}, d}^{(1)} \times \ln H_{c_{1}}^{0}+\Delta_{d^{\prime}, d}^{(2)}+o(1) .
$$

If $d>d^{\prime} \geqslant 0$ then

$$
h_{\mathrm{ex}} \geqslant H_{c_{1}}^{0}+\Delta_{d, d^{\prime}}^{(1)} \times \ln H_{c_{1}}^{0}+\Delta_{d, d^{\prime}}^{(2)}+o(1) .
$$

We are now in position to give an asymptotic value for the first critical field. Indeed with Corollary $10.3\left((10.3)\right.$ with $d=0$ and $d^{\prime} \in\left\{1, \ldots, N_{0}\right\}$ and (10.4) with $d \geqslant 1$ and $\left.d^{\prime}=0\right)$.

COROLlary 10.4. - Denote $H_{c_{1}}:=H_{c_{1}}^{0}+\min _{d \in\left\{1, \ldots, N_{0}\right\}} \frac{\overline{\mathcal{W}}_{d}}{d M_{\Omega}}$. Let $\left\{\left(v_{\varepsilon}, A_{\varepsilon}\right) \mid 0<\varepsilon<1\right\} \subset \mathscr{H}$ be a family of quasi-minimizers satisfying (3.11).

(1) If for sufficiently small $\varepsilon$ we have $d=0$ then $h_{\mathrm{ex}} \leqslant H_{c_{1}}+o(1)$.

(2) If for sufficiently small $\varepsilon$ we have $d>0$ then $h_{\mathrm{ex}} \geqslant H_{c_{1}}+o(1)$.

Proof. - The corollary is a direct consequence of Corollary 10.3 taking $d^{\prime} \in\left\{1, \ldots, N_{0}\right\}$ which minimizes $\Delta_{d^{\prime}, 0}^{(2)}=W_{d^{\prime}} /\left(M_{\Omega} d^{\prime}\right)$ in (10.3) for the first assertion and $d^{\prime}=0$ in (10.4) for the second.

\subsection{Secondary critical fields for $d \in\left\{1, \ldots, N_{0}\right\}$}

If $N_{0}=1$, if $h_{\mathrm{ex}}$ is near $H_{c_{1}}$ and if $d>0$, then it is standard to prove that $d=1$. If $N_{0} \geqslant 2$ and $d \in\left\{1, \ldots, N_{0}\right\}$, then the situation is more involved: we have no a priori sharp informations about the number of vorticity defects and their (macroscopic) location. The goal of this section is to get such informations.

\subsubsection{Preliminaries}

Note that for $0 \leqslant d<d^{\prime} \leqslant N_{0}$ we have $\Delta_{d^{\prime}, d}^{(1)}=0$ and $\Delta_{d^{\prime}, d}^{(2)}=\frac{\overline{\mathcal{W}}_{d^{\prime}}-\overline{\mathcal{W}}_{d}}{M_{\Omega}\left(d^{\prime}-d\right)}$.

Rephrasing Corollary 10.3 for $d, d^{\prime} \in\left\{0, \ldots, N_{0}\right\}$ we have the following key lemma.

LEMma 10.5. - Let $\varepsilon=\varepsilon_{n} \downarrow 0, \lambda, \delta, h_{\mathrm{ex}}$ and $\left(\left(v_{\varepsilon}, A_{\varepsilon}\right)\right)_{\varepsilon} \subset \mathscr{H}$ be as in Corollary 10.1. hold:

Assume $\operatorname{Card}(\mathcal{Z})=d$ is independent of $\varepsilon$ then the following properties 
(1) If $0 \leqslant d^{\prime}<d$ then, letting $\overline{\mathcal{W}}_{0}:=0$, we have $h_{\mathrm{ex}} \geqslant H_{c_{1}}^{0}+\frac{\overline{\mathcal{W}}_{d}-\overline{\mathcal{W}}_{d^{\prime}}}{M_{\Omega}\left(d-d^{\prime}\right)}+$ $o(1)$. In particular taking $d^{\prime}=0$ we get $h_{\mathrm{ex}} \geqslant H_{c_{1}}^{0}+\frac{\overline{\mathcal{W}}_{d}}{M_{\Omega} d}+o(1)$.

(2) If $d<N_{0}$ and $d<d^{\prime} \leqslant N_{0}$ then $h_{\mathrm{ex}} \leqslant H_{c_{1}}^{0}+\frac{\overline{\mathcal{W}}_{d^{\prime}}-\overline{\mathcal{W}}_{d}}{M_{\Omega}\left(d^{\prime}-d\right)}+o(1)$.

(3) If $N_{0} \geqslant 2, N_{0} \geqslant d^{\prime}>d \geqslant 1$ then

$\frac{\overline{\mathcal{W}}_{d^{\prime}}}{d^{\prime}}<\frac{\overline{\mathcal{W}}_{d^{\prime}}-\overline{\mathcal{W}}_{d}}{d^{\prime}-d} \Longleftrightarrow \frac{\overline{\mathcal{W}}_{d}}{d}<\frac{\overline{\mathcal{W}}_{d^{\prime}}}{d^{\prime}}$ and $\frac{\overline{\mathcal{W}}_{d^{\prime}}}{d^{\prime}}>\frac{\overline{\mathcal{W}}_{d^{\prime}}-\overline{\mathcal{W}}_{d}}{d^{\prime}-d} \Longleftrightarrow \frac{\overline{\mathcal{W}}_{d}}{d}>\frac{\overline{\mathcal{W}}_{d^{\prime}}}{d^{\prime}}$

(4) If $N_{0} \geqslant 2$ and $N_{0} \geqslant d^{\prime}>d \geqslant 1$ then

$$
\frac{\overline{\mathcal{W}}_{d^{\prime}}}{d^{\prime}}=\frac{\overline{\mathcal{W}}_{d^{\prime}}-\overline{\mathcal{W}}_{d}}{d^{\prime}-d} \Longleftrightarrow \frac{\overline{\mathcal{W}}_{d}}{d}=\frac{\overline{\mathcal{W}}_{d^{\prime}}}{d^{\prime}} \text {. }
$$

(5) If $N_{0} \geqslant 2$ and $0 \leqslant d<d^{\prime}<d^{\prime \prime} \leqslant N_{0}$ then we have the following convex combination

$$
\begin{gathered}
\frac{\overline{\mathcal{W}}_{d^{\prime \prime}}-\overline{\mathcal{W}}_{d}}{d^{\prime \prime}-d}=\frac{d^{\prime \prime}-d^{\prime}}{d^{\prime \prime}-d} \frac{\overline{\mathcal{W}}_{d^{\prime \prime}}-\overline{\mathcal{W}}_{d^{\prime}}}{d^{\prime \prime}-d^{\prime}}+\frac{d^{\prime}-d}{d^{\prime \prime}-d} \frac{\overline{\mathcal{W}}_{d^{\prime}}-\overline{\mathcal{W}}_{d}}{d^{\prime}-d} . \\
\text { Consequenlty } \frac{\overline{\mathcal{W}}_{d^{\prime \prime}-\overline{\mathcal{W}}_{d}}}{d^{\prime \prime}-d} \text { is between } \frac{\overline{\mathcal{W}}_{d^{\prime \prime}}-\overline{\mathcal{W}}_{d^{\prime}}}{d^{\prime \prime}-d^{\prime}} \text { and } \frac{\overline{\mathcal{W}}_{d^{\prime}}-\overline{\mathcal{W}}_{d}}{d^{\prime}-d} .
\end{gathered}
$$

Proof. - The two first assertions are obtained with Corollary 10.3. The remaining part of the lemma consists in basic calculations.

\subsubsection{First step in the definition of the critical fields}

Assume $N_{0} \geqslant 2$. We are going to define some energetic levels (in term of $\overline{\mathcal{W}}_{d}$ ) related with the number of vorticity defects and their (macroscopic) location.

We denote $d_{0}^{\star}:=0, \mathscr{S}_{1}:=\left\{1, \ldots, N_{0}\right\}, \mathscr{K}_{1}^{\star}:=\min _{d \in \mathscr{S}_{1}} \frac{\overline{\mathcal{W}}_{d}}{d}=$ $\min _{d \in \mathscr{S}_{1}} \frac{\overline{\mathcal{W}}_{d}-\overline{\mathcal{W}}_{d_{0}^{\star}}}{d-d_{0}^{\star}}, \mathscr{S}_{1}^{\star}:=\left\{d \in \mathscr{S}_{1} \mid \overline{\mathcal{W}}_{d} / d=\mathscr{K}_{1}^{\star}\right\}$ and $\mathscr{D}_{1}:=\left\{\mathbf{D} \in \Lambda_{d} \mid d \in\right.$ $\mathscr{S}_{1}^{\star}$ and $\mathbf{D}$ minimizes $\left.\mathcal{W}_{d}\right\}$. We let also $d_{1}^{\star}:=\max \mathscr{S}_{1}^{\star}$ and $\mathscr{D}_{1}^{\star}:=\mathscr{D}_{1} \cap \Lambda_{d_{1}^{\star}}$.

If $d_{1}^{\star}=N_{0}$ we are going to prove that for $h_{\mathrm{ex}} \geqslant H_{c_{1}}+o(1)$ (but $h_{\mathrm{ex}}$ not too large), then there is exactly one vorticity defect close to each point of $\Lambda$. In the contrary case $\left(1 \leqslant d_{1}^{\star}<N_{0}\right)$, then there are other critical fields which govern the number of vorticity defects.

If $d_{1}^{\star}<N_{0}$, then $\mathscr{S}_{2}:=\left\{d_{1}^{\star}+1, \ldots, N_{0}\right\} \neq \emptyset$. For $d \in \mathscr{S}_{2}$ we let $\mathscr{K}_{2}(d):=$ $\frac{\overline{\mathcal{W}}_{d}-\overline{\mathcal{W}}_{d_{1}^{\star}}}{d-d_{1}^{\star}}, \mathscr{S}_{2}^{\star}:=\left\{d \in \mathscr{S}_{2} \mid \mathscr{K}_{2}(d)=\min _{\mathscr{S}_{2}} \mathscr{K}_{2}\right\}, d_{2}^{\star}:=\max \mathscr{S}_{2}^{\star}$ and $\mathscr{K}_{2}^{\star}:=$ $\mathscr{K}_{2}\left(d_{2}^{\star}\right)$.

We denote $\mathscr{D}_{2}:=\left\{\mathbf{D} \in \Lambda_{d} \mid d \in \mathscr{S}_{2}^{\star}\right.$ and $\mathbf{D}$ minimizes $\left.\mathcal{W}_{d}\right\}$ and $\mathscr{D}_{2}^{\star}:=$ $\mathscr{D}_{2} \cap \Lambda_{d_{2}^{\star}}$. 
We claim that for $d \in \mathscr{S}_{2}$ we have $\overline{\mathcal{W}}_{d} / d>\overline{\mathcal{W}}_{d_{1}^{\star}} / d_{1}^{\star}$. Then, with Lemma $10.5(3)$, we get $\mathscr{K}_{2}(d)>\overline{\mathcal{W}}_{d_{1}^{\star}} / d_{1}^{\star}$. In particular

$$
\mathscr{K}_{2}^{\star}=\frac{\overline{\mathcal{W}}_{d_{2}^{\star}}-\overline{\mathcal{W}}_{d_{1}^{\star}}}{d_{2}^{\star}-d_{1}^{\star}}>\frac{\overline{\mathcal{W}}_{d_{1}^{\star}}}{d_{1}^{\star}}=\mathscr{K}_{1}^{\star}
$$

If $d_{2}^{\star}=N_{0}$ then we stop the construction. In the contrary case, for $d \in \mathscr{S}_{3}:=$ $\left\{d_{2}^{\star}+1, \ldots, N_{0}\right\} \neq \emptyset$ we have $\mathscr{K}_{2}(d)>\mathscr{K}_{2}\left(d_{2}^{\star}\right)$.

We continue the iterative construction. For $k \geqslant 2$, assume that we have $1<d_{k-1}^{\star}<d_{k}^{\star}<N_{0}$, we let $\mathscr{S}_{k+1}:=\left\{d_{k}^{\star}+1, \ldots, N_{0}\right\} \neq \emptyset$ and we assume that for $d \in \mathscr{S}_{k+1}$ :

$$
\mathscr{K}_{k}(d):=\frac{\overline{\mathcal{W}}_{d}-\overline{\mathcal{W}}_{d_{k-1}^{\star}}}{d-d_{k-1}^{\star}}>\frac{\overline{\mathcal{W}}_{d_{k}^{\star}}-\overline{\mathcal{W}}_{d_{k-1}^{\star}}}{d_{k}^{\star}-d_{k-1}^{\star}}=\mathscr{K}_{k}^{\star} .
$$

For $d \in \mathscr{S}_{k+1}$ we let $\mathscr{K}_{k+1}(d):=\frac{\overline{\mathcal{W}}_{d}-\overline{\mathcal{W}}_{d_{k}^{\star}}}{d-d_{k}^{\star}}$,

$$
\begin{array}{r}
\mathscr{S}_{k+1}^{\star}:=\left\{d \in \mathscr{S}_{k+1} \mid \mathscr{K}_{k+1}(d)=\min _{\mathscr{S}_{k+1}} \mathscr{K}_{k+1}\right\}, \\
d_{k+1}^{\star}:=\max \mathscr{S}_{k+1}^{\star} \text { and } \mathscr{K}_{k+1}^{\star}:=\mathscr{K}_{k+1}\left(d_{k+1}^{\star}\right) .
\end{array}
$$

We define also

$$
\begin{aligned}
\mathscr{D}_{k+1} & :=\left\{\mathbf{D} \mid \mathbf{D} \in \Lambda_{d}, d \in \mathscr{S}_{k+1}^{\star} \text { and } \mathbf{D} \text { minimizes } \mathcal{W}_{d}\right\} \\
\text { and } \mathscr{D}_{k+1}^{\star} & :=\mathscr{D}_{k+1} \cap \Lambda_{d_{k+1}^{\star}} .
\end{aligned}
$$

From (10.6) we have

$$
\mathscr{K}_{k}\left(d_{k+1}^{\star}\right)=\frac{\overline{\mathcal{W}}_{d_{k+1}^{\star}}-\overline{\mathcal{W}}_{d_{k-1}^{\star}}}{d_{k+1}^{\star}-d_{k-1}^{\star}}>\frac{\overline{\mathcal{W}}_{d_{k}^{\star}}-\overline{\mathcal{W}}_{d_{k-1}^{\star}}}{d_{k}^{\star}-d_{k-1}^{\star}}=\mathscr{K}_{k}^{\star} .
$$

Then, from Lemma 10.5(5) with $d=d_{k-1}^{\star}, d^{\prime}=d_{k}^{\star}$ and $d^{\prime \prime}=d_{k+1}^{\star}$, we get that $\mathscr{K}_{k}\left(d_{k+1}^{\star}\right)$ is between $\mathscr{K}_{k}^{\star}$ and $\mathscr{K}_{k+1}^{\star}$. Consequently, with (10.7) we get

$$
\mathscr{K}_{k+1}^{\star}>\mathscr{K}_{k}^{\star} \text {. }
$$

We stop the construction at Step $L$ s.t. $d_{L}^{\star}=N_{0}$. Since $1 \leqslant d_{k}^{\star}<d_{k+1}^{\star} \leqslant N_{0}$, it is clear that a such $L$ exists and $1 \leqslant L \leqslant N_{0}$.

We then have two possibilities: $L=1$ or $L \in\left\{2, \ldots, N_{0}\right\}$. If $L \geqslant 2$ then, for $k \in\{1, \ldots, L-1\},(10.8)$ holds. We also claim that $(1, \ldots, 1) \in \mathscr{D}_{L}$.

LEMMA 10.6. - Let $k \in\{1, \ldots, L\}$, assume that $d_{k}^{\star}-d_{k-1}^{\star} \geqslant 2$ and fix $d_{k-1}^{\star}<d<d_{k}^{\star}$. We have

$$
\frac{\overline{\mathcal{W}}_{d_{k}^{\star}}-\overline{\mathcal{W}}_{d}}{d_{k}^{\star}-d} \leqslant \mathscr{K}_{k}^{\star} \leqslant \frac{\overline{\mathcal{W}}_{d}-\overline{\mathcal{W}}_{d_{k-1}^{\star}}}{d-d_{k-1}^{\star}}
$$


Moreover, if $d \notin \mathscr{S}_{k}^{\star}$, then

$$
\frac{\overline{\mathcal{W}}_{d_{k}^{\star}}-\overline{\mathcal{W}}_{d}}{d_{k}^{\star}-d} \leqslant \mathscr{K}_{k}^{\star}<\frac{\overline{\mathcal{W}}_{d}-\overline{\mathcal{W}}_{d_{k-1}^{\star}}}{d-d_{k-1}^{\star}} .
$$

Proof. - From Lemma 10.5 (5), $\mathscr{K}_{k}^{\star}$ is between $\frac{\overline{\mathcal{W}}_{d}-\overline{\mathcal{W}}_{d_{k-1}^{\star}}}{d-d_{k-1}^{\star}}$ and $\frac{\overline{\mathcal{W}}_{d_{k}^{\star}}-\overline{\mathcal{W}}_{d}}{d_{k}^{\star}-d}$. On the other hand, from the definition of $d_{k}^{\star}, \mathscr{K}_{k}^{\star} \leqslant \frac{\overline{\mathcal{W}}_{d}-\overline{\mathcal{W}}_{d_{k-1}^{\star}}}{d-d_{k-1}^{\star}}$. Clearly the first part of the lemma holds. If $d \notin \mathscr{S}_{k}^{\star}$ then, by definition, $\mathscr{K}_{k}^{\star}<$ $\frac{\overline{\mathcal{W}}_{d}-\overline{\mathcal{W}}_{d_{k-1}^{\star}}}{d-d_{k-1}^{\star}}$.

\subsubsection{Main result}

For $k \in\{1, \ldots, L\}$ we let

$$
\mathrm{K}_{k}^{(\mathrm{I})}:=H_{c_{1}}^{0}+\frac{\mathscr{K}_{k}^{\star}}{M_{\Omega}}
$$

and we let also

$$
\mathrm{K}_{1}^{(\mathrm{II})}:=H_{c_{1}}^{0}+\Delta_{N_{0}}^{(1)} \times \ln H_{c_{1}}^{0}+\Delta_{N_{0}}^{(2)} .
$$

Recall that the $\mathscr{K}_{k}^{\star}$ 's are defined in Section 10.1.2 and $\Delta_{N_{0}}^{(1)}$ and $\Delta_{N_{0}}^{(2)}$ in Lemma 10.2. Note that $H_{c_{1}}=\mathrm{K}_{1}^{(\mathrm{I})}$.

Proposition 10.7. - Assume that (1.5) holds and $\lambda, \delta, h_{\mathrm{ex}}, K$ satisfy (1.2), (1.3) and (1.4).

Let $\left\{\left(v_{\varepsilon}, A_{\varepsilon}\right) \mid 0<\varepsilon<1\right\} \subset \mathscr{H}$ be a family satisfying (3.11) and (8.1) which is in the Coulomb gauge. Assume $d_{\varepsilon}=\operatorname{Card}\left(\mathcal{Z}_{\varepsilon}\right) \in\left\{1, \ldots, N_{0}\right\}$.

We denote $\mathbf{D}=\left(D_{1}, \ldots, D_{N_{0}}\right)$ with $D_{l}=\operatorname{deg}_{\partial B\left(p_{l}, \eta_{\Omega}\right)}(v)$ ( $\eta_{\Omega}$ is defined in (6.14)).

(1) Assume $L=1$. For sufficiently small $\varepsilon>0$ we have $\mathbf{D} \in \mathscr{D}_{1}$.

Moreover, if $\varepsilon=\varepsilon_{n} \downarrow 0$ is a sequence s.t. $d_{\varepsilon}$ is independent of $\varepsilon$ and $d_{\varepsilon} \neq N_{0}$ (i.e. $\mathbf{D} \neq(1, \ldots, 1)$ ) then $h_{\mathrm{ex}} \leqslant \mathrm{K}_{1}^{(\mathrm{I})}+o(1)$.

(2) Assume $L \geqslant 2$. For $k \in\{1, \ldots, L-1\}$, if $d_{k-1}^{\star}<d_{\varepsilon} \leqslant d_{k}^{\star}$ for small $\varepsilon$ or for a sequence indexed by $\varepsilon=\varepsilon_{n} \downarrow 0$, then

$$
\mathrm{K}_{k}^{(\mathrm{I})}+o(1) \leqslant h_{\mathrm{ex}} \leqslant \mathrm{K}_{k+1}^{(\mathrm{I})}+o(1) .
$$

Moreover, for sufficiently small $\varepsilon, \mathbf{D} \in \mathscr{D}_{k}$. And if $\mathbf{D} \in \mathscr{D}_{k} \backslash \mathscr{D}_{k}^{\star}$ (i.e. $d_{k-1}^{\star}<d_{\varepsilon}<d_{k}^{\star}$ ) then

$$
h_{\mathrm{ex}} \leqslant \mathrm{K}_{k}^{(\mathrm{I})}+o(1) .
$$


(3) If $d_{L-1}^{\star}<d_{\varepsilon} \leqslant d_{L}^{\star}=N_{0}$ for small $\varepsilon$ or for a sequence indexed by $\varepsilon=\varepsilon_{n} \downarrow 0$, then

$$
\mathrm{K}_{L}^{(\mathrm{I})}+o(1) \leqslant h_{\mathrm{ex}} \leqslant \mathrm{K}_{1}^{(\mathrm{II})}+o(1) .
$$

Moreover, for sufficiently small $\varepsilon, \mathbf{D} \in \mathscr{D}_{L}$. And if $d_{\varepsilon}<N_{0}$ (i.e $\mathbf{D} \neq(1, \ldots, 1))$ then

$$
h_{\mathrm{ex}} \leqslant \mathrm{K}_{L}^{(\mathrm{I})}+o(1) .
$$

In particular, for sufficiently small $\varepsilon$, we have $\mathbf{D} \in \bigcup_{l=1}^{L} \mathscr{D}_{l}$.

Proof. - We prove the first item arguing by contradiction. First note that if $N_{0}=1$ then there is nothing to prove. Assume thus $N_{0} \geqslant 2$ and $L=1$ and let $\left\{\left(v_{\varepsilon}, A_{\varepsilon}\right) \mid 0<\varepsilon<1\right\}$ be as in the proposition. Assume there exists $\varepsilon=\varepsilon_{n} \downarrow 0$ s.t. $\mathbf{D} \notin \mathscr{D}_{1}$. Up to a subsequence we may assume that $\mathbf{D}$ is independent of $\varepsilon$.

From Corollary 10.1, for sufficiently small $\varepsilon, \mathbf{D}$ minimizes $\mathcal{W}_{d}$ and then, from the definition of $\mathscr{D}_{1}$, we get $d \notin \mathscr{S}_{1}^{\star}$. Consequently $\overline{\mathcal{W}}_{N_{0}} / N_{0}<\overline{\mathcal{W}}_{d} / d$ and thus, from Lemma 10.5(2) and 10.5(3) (with $d^{\prime}=N_{0}$ ), we get the existence of $t>0$ s.t. $h_{\mathrm{ex}} \leqslant H_{c_{1}}-t$. This last estimate is in contradiction with Corollary 10.4(2). Thus $\mathbf{D} \in \mathscr{D}_{1}$ for sufficiently small $\varepsilon$. The rest of the first assertion is a direct consequence of $d \in \mathscr{S}_{1}^{\star} \backslash\left\{N_{0}\right\}$ and Lemma 10.5(2) and 10.5 (4) (with $d^{\prime}=N_{0}$ ).

We now prove the second assertion. Assume $L \geqslant 2$. For $k \in\{1, \ldots$, $L-1\}$, if $d_{k-1}^{\star}<d \leqslant d_{k}^{\star}$, then, from Lemma 10.5(1) (with $d^{\prime}=d_{k-1}^{\star}$ ) and Lemma 10.5(2) (with $d^{\prime}=d_{k+1}^{\star}$ ), we get

$$
\frac{\overline{\mathcal{W}}_{d}-\overline{\mathcal{W}}_{d_{k-1}^{\star}}}{M_{\Omega}\left(d-d_{k-1}^{\star}\right)}+o(1) \leqslant h_{\mathrm{ex}}-H_{c_{1}}^{0} \leqslant \frac{\overline{\mathcal{W}}_{d_{k+1}^{\star}}-\overline{\mathcal{W}}_{d}}{M_{\Omega}\left(d_{k+1}^{\star}-d\right)}+o(1) \text {. }
$$

From the definition of $d_{k}^{\star}$ we have $\mathscr{K}_{k}^{\star} \leqslant \frac{\overline{\mathcal{W}}_{d}-\overline{\mathcal{W}}_{d_{k-1}^{\star}}}{d-d_{k-1}^{\star}}$ and then the lower bound in (10.15) gives the first convergence in (10.11).

On the other hand, if $d=d_{k}^{\star}$ then, from the definition of $\mathscr{K}_{k+1}^{\star}$, the upper bound in (10.15) gives the second convergence in (10.11).

If $d \neq d_{k}^{\star}$, using Lemma 10.5(5) (with $d<d_{k}^{\star}<d_{k+1}^{\star}$ ) we obtain that $\frac{\overline{\mathcal{W}}_{d_{k+1}^{\star}}-\overline{\mathcal{W}}_{d}}{d_{k+1}^{\star}-d}$ is between $\frac{\overline{\mathcal{W}}_{d_{k}^{\star}}-\overline{\mathcal{W}}_{d}}{d_{k}^{\star}-d}$ and $\mathscr{K}_{k+1}^{\star}$. But, from Lemma 10.6, we get $\frac{\overline{\mathcal{W}}_{d_{k}}-\overline{\mathcal{W}}_{d}}{d_{k}^{\star}-d} \leqslant \mathscr{K}_{k}^{\star}$. Since from (10.8) we have $\mathscr{K}_{k+1}^{\star}>\mathscr{K}_{k}^{\star}$, we obtain $\frac{\overline{\mathcal{W}}_{d_{k+1}^{\star}}-\overline{\mathcal{W}}_{d}}{d_{k+1}^{\star}-d} \leqslant \mathscr{K}_{k+1}^{\star}$. Therefore the upper bound of (10.15) gives the second convergence in (10.11). 
We now demonstrate that, for sufficiently small $\varepsilon, \mathbf{D} \in \mathscr{D}_{k}$ arguing by contradiction. We assume the existence of sequence $\varepsilon=\varepsilon_{n} \downarrow 0$ s.t. $d_{k-1}^{\star}<$ $d \leqslant d_{k}^{\star}$ with $k \in\{1, \ldots, L-1\}, \mathbf{D}$ is independent of $\varepsilon$ and $\mathbf{D} \notin \mathscr{D}_{k}$. From Corollary 10.1, D minimizes $\mathcal{W}_{d}$ and then, from the definition of $\mathscr{D}_{k}$, we get $d \notin \mathscr{S}_{k}^{\star}\left(\right.$ then $\left.d<d_{k}^{\star}\right)$.

On the one hand, with Lemma 10.5(1) (with $d^{\prime}=d_{k-1}^{\star}$ ) and Lemma 10.5(2) (with $d^{\prime}=d_{k}^{\star}$ ) we have

$$
\frac{\overline{\mathcal{W}}_{d}-\overline{\mathcal{W}}_{d_{k-1}^{\star}}}{M_{\Omega}\left(d-d_{k-1}^{\star}\right)}+o(1) \leqslant h_{\mathrm{ex}}-H_{c_{1}}^{0} \leqslant \frac{\overline{\mathcal{W}}_{d}-\overline{\mathcal{W}}_{d_{k}^{\star}}}{M_{\Omega}\left(d-d_{k}^{\star}\right)}+o(1)
$$

On the other hand, with Lemma 10.6, we have $\frac{\overline{\mathcal{W}}_{d}-\overline{\mathcal{W}}_{d_{k}^{\star}}}{d-d_{k}^{\star}}<\frac{\overline{\mathcal{W}}_{d}-\overline{\mathcal{W}}_{d_{k-1}^{\star}}}{d-d_{k-1}^{\star}}$. This inequality gives a contradiction.

Lemma 10.5 (2) (with $d^{\prime}=d_{k}^{\star}$ ) and Lemma 10.6 give immediately (10.12).

We now treat the last item of the proposition and we assume $d_{L-1}^{\star}<d \leqslant$ $d_{L}^{\star}=N_{0}$. From (10.4) (with $d^{\prime}=d_{L-1}^{\star}$ ) we get $h_{\mathrm{ex}}-H_{c_{1}}^{0} \geqslant \Delta_{d, d_{L-1}^{\star}}^{(2)}+o(1)$. On the other hand, from the definition of $\mathscr{K}_{L}^{\star}$, we get

$$
h_{\mathrm{ex}}-H_{c_{1}}^{0} \geqslant \frac{\mathscr{K}_{L}^{\star}}{M_{\Omega}}+o(1) .
$$

Before ending the proof of (10.13) we prove that (10.14) holds and, for sufficiently small $\varepsilon, \mathbf{D} \in \mathscr{D}_{L}$. Assume that there exists $\varepsilon=\varepsilon_{n} \downarrow 0$ s.t. $\mathbf{D}$ is independent of $\varepsilon$ and $d_{L-1}^{\star}<d<N_{0}$.

From Lemma 10.5(2) (with $d^{\prime}=N_{0}$ ) we have

$$
h_{\mathrm{ex}}-H_{c_{1}}^{0} \leqslant \frac{\overline{\mathcal{W}}_{N_{0}}-\overline{\mathcal{W}}_{d}}{M_{\Omega}\left(N_{0}-d\right)}+o(1) .
$$

Using (10.16) with (10.17) we get $\mathscr{K}_{L}^{\star} \leqslant\left(\overline{\mathcal{W}}_{N_{0}}-\overline{\mathcal{W}}_{d}\right) /\left(N_{0}-d\right)$. Lemma 10.6 (with $\left.d_{L-1}^{\star}<d<N_{0}\right)$ gives $\left(\overline{\mathcal{W}}_{N_{0}}-\overline{\mathcal{W}}_{d}\right) /\left(N_{0}-d\right) \leqslant \mathscr{K}_{L}^{\star}$. Therefore, $\left(\overline{\mathcal{W}}_{N_{0}}-\overline{\mathcal{W}}_{d}\right) /\left(N_{0}-d\right)=\mathscr{K}_{L}^{\star}$ and then by combining (10.16) and (10.17) we deduce that, if for some sequence $\varepsilon=\varepsilon_{n} \downarrow 0$ we have $d_{L-1}^{\star}<d<N_{0}$, then (10.14) holds.

Arguing as above, (using (10.2) with $d_{0}=N_{0}$ ), one may prove that for sufficiently small $\varepsilon$ we have $d \in \mathscr{S}_{L}^{\star}$ and thus $\mathbf{D} \in \mathscr{D}_{L}$.

We complete the proof of (10.13). Assume that $h_{\mathrm{ex}}$ is sufficiently large in order to have $d=N_{0}$ (here we used (10.14)). It suffices to use (10.3) (with $d=N_{0}$ and $\left.d^{\prime}=N_{0}+1\right)$ in order to get the remaining part of (10.13). 


\subsection{Secondary critical fields for $d \geqslant N_{0}+1$}

The case $d \geqslant N_{0}+1$ is easier to handle than the case $1 \leqslant d \leqslant N_{0}$.

For $k \in \mathbb{N}^{*}$, we let

$$
\mathrm{K}_{k}^{(\mathrm{II})}:=H_{c_{1}}^{0}+\Delta_{N_{0}+k}^{(1)} \times \ln H_{c_{1}}^{0}+\Delta_{N_{0}+k}^{(2)}
$$

where $\Delta_{N_{0}+k}^{(1)}$ and $\Delta_{N_{0}+k}^{(2)}$ are defined in Lemma 10.2. We have the following proposition.

Proposition 10.8. - Assume that (1.5) holds and $\lambda, \delta, h_{\mathrm{ex}}, K$ satisfy (1.2), (1.3) and (1.4).

Let $\left\{\left(v_{\varepsilon}, A_{\varepsilon}\right) \mid 0<\varepsilon<1\right\} \subset \mathscr{H}$ be a family satisfying (3.11) and (8.1) which is in the Coulomb gauge.

Let $k \in \mathbb{N}^{*}$. If for a sequence $\varepsilon=\varepsilon_{n} \downarrow 0$ we have $d_{\varepsilon}=N_{0}+k$ then

$$
\mathrm{K}_{k}^{(\mathrm{II})}+o(1) \leqslant h_{\mathrm{ex}} \leqslant \mathrm{K}_{k+1}^{(\mathrm{II})}+o(1) .
$$

Proof. - The proposition is a direct consequence of (10.3) (with $d=$ $N_{0}+k$ and $d^{\prime}=N_{0}+k+1$ ) and (10.4) (with $d=N_{0}+k$ and $d^{\prime}=$ $\left.N_{0}+k-1\right)$.

\section{Appendix A. Proof of Estimate (5.9)}

Consider a conformal mapping $\Phi: \mathbb{D} \rightarrow \Omega$. From a result of Painlevé (see Footnote (4) on p. 716), the maps $\Phi$ and $\Phi^{-1}$ may be extended in $\bar{\Omega}$ and $\overline{\mathbb{D}}$ by smooth maps. Then there exists $C_{\star} \geqslant 1$ s.t.

$$
\|\nabla \Phi\|_{L^{\infty}(\mathbb{D})},\left\|\nabla \Phi^{-1}\right\|_{L^{\infty}(\Omega)} \leqslant C_{\star} .
$$

Write $\widetilde{a}_{\varepsilon}:=a_{\varepsilon} \circ \Phi$ and $\widetilde{U}_{\varepsilon}:=U_{\varepsilon} \circ \Phi$. Since the function $\widetilde{U}_{\varepsilon}$ is a minimizers of $\widetilde{E}_{\varepsilon}$, the analog of $E_{\varepsilon}$ in $\mathbb{D}, \widetilde{U}_{\varepsilon}$ is a solution of

$$
\begin{cases}-\Delta \widetilde{U}=w \frac{\tilde{U}}{\varepsilon^{2}}\left(\widetilde{a}_{\varepsilon}^{2}-|\widetilde{U}|^{2}\right) & \text { in } \mathbb{D} \\ \partial_{\nu} \widetilde{U}=0 & \text { on } \mathbb{S}^{1}\end{cases}
$$

with $w=\operatorname{Jac} \Phi$ is the Jacobian of $\Phi$.

Define $V_{\varepsilon}: B(0,2) \rightarrow\left[b^{2}, 1\right]$ by

$$
V_{\varepsilon}(x)= \begin{cases}\widetilde{U}_{\varepsilon}(x) & \text { if } x \in \mathbb{D} \\ \widetilde{U}_{\varepsilon}\left(x /|x|^{2}\right) & \text { if } x \in B(0,2) \backslash \mathbb{D} .\end{cases}
$$

Then $-\Delta V_{\varepsilon}=-\Delta \widetilde{U}_{\varepsilon}$ in $\mathbb{D}$ and $-\Delta V_{\varepsilon}(x)=-|x|^{-4} \Delta \widetilde{U}_{\varepsilon}\left(x /|x|^{2}\right)$ in $B(0,2) \backslash \mathbb{D}$. Thus $V_{\varepsilon} \in H^{2}(B(0,2), \mathbb{C})$. 
First note that if $r \leqslant \varepsilon$, then (5.9) is given by (5.7).

Let $r>\varepsilon$ and $x_{0} \in \Omega$ be s.t. $\operatorname{dist}\left(x_{0}, \partial \omega_{\varepsilon}\right)>r$. Let $\eta:=a_{\varepsilon}\left(x_{0}\right)-V_{\varepsilon}$ in $B\left(x_{0}, r / 2\right)$. From Lemma A.1 in [3] and (5.8) we get, for $x \in B\left(x_{0}, r / 4\right)$, $\left|\nabla V_{\varepsilon}(x)\right|^{2}=|\nabla \eta(x)|^{2}$ where

$$
\begin{aligned}
|\nabla \eta(x)|^{2} & \leqslant C\left(\|\Delta \eta\|_{L^{\infty}\left(B\left(x_{0}, r / 2\right)\right)}+\frac{4}{r^{2}}\|\eta\|_{L^{\infty}\left(B\left(x_{0}, r / 2\right)\right)}\right)\|\eta\|_{L^{\infty}\left(B\left(x_{0}, r / 2\right)\right)} \\
& \leqslant \frac{C \mathrm{e}^{-\frac{s_{b} r}{2 \varepsilon}}}{\varepsilon^{2}} .
\end{aligned}
$$

In the previous estimate the constants are independent of $\varepsilon, r$ and $x_{0}$. From (A.1) we then get (5.9).

\section{Appendix B. Proof of Theorem 5.6}

Assume that $\lambda, \delta, h_{\mathrm{ex}}, K$ satisfy (1.2), (1.3) and $\delta^{2}|\ln \varepsilon| \leqslant 1$.

Consider a family of configurations $\left\{\left(v_{\varepsilon}, A_{\varepsilon}\right) \mid 0<\varepsilon<1\right\} \subset \mathscr{H}$ which is in the Coulomb gauge and s.t.

$$
\mathcal{F}\left(v_{\varepsilon}, A_{\varepsilon}\right) \leqslant \inf _{\mathscr{H}} \mathcal{F}+\mathcal{O}(\ln |\ln \varepsilon|) .
$$

We drop the subscript $\varepsilon$. From Lemma 5.8, we may consider $A_{v} \in H^{1}\left(\Omega, \mathbb{R}^{2}\right)$ s.t. $\left(v, A_{v}\right)$ is in the Coulomb gauge and (5.22) holds.

We then have

$$
\mathcal{F}\left(v, A_{v}\right) \leqslant \mathcal{F}(v, A) \leqslant \inf _{\mathscr{H}} \mathcal{F}+\mathcal{O}(\ln |\ln \varepsilon|)=\mathcal{O}\left(h_{\text {ex }}^{2}\right) .
$$

Proposition 5.5 gives the existence of $C, \varepsilon_{0}>0$ (independent of $\varepsilon$ ) s.t., for $\varepsilon<\varepsilon_{0}$, there exists a family of disjoint disks $\left\{B_{i} \mid i \in \mathcal{J}\right\}$ with $B_{i}=B\left(a_{i}, r_{i}\right)$ satisfying:

(1) $\left\{|v|<1-|\ln \varepsilon|^{-2}\right\} \subset \bigcup B_{i}$

(2) $\sum r_{i}<|\ln \varepsilon|^{-10}$,

(3) writing $\rho=|v|$ and $v=\rho \mathrm{e}^{\imath \varphi}$ we have

$$
\frac{1}{2} \int_{B_{i}} \rho^{2}|\nabla \varphi-A|^{2}+\left|\operatorname{curl}(A)-h_{\mathrm{ex}}\right|^{2} \geqslant \pi\left|d_{i}\right|(|\ln \varepsilon|-C \ln |\ln \varepsilon|),
$$

where $d_{i}=\operatorname{deg}_{\partial B_{i}}(v)$ if $B_{i} \subset \Omega$ and 0 otherwise.

From now on, the notation $C$ stands for a positive constant independent of $\varepsilon$ whose value may change from one line to another. 


\section{B.1. A substitution lemma}

As in [15], we first state a substitution lemma.

Lemma B.1. - There exists $(\widetilde{v}, \widetilde{A}) \in \mathscr{H}$ which is in the Coulomb gauge and s.t., writing, $\rho=|v|, v=\rho \mathrm{e}^{\imath \varphi}$ and $\widetilde{\rho}=|\widetilde{v}|, \widetilde{v}=\widetilde{\rho} \mathrm{e}^{\imath \tilde{\varphi}}$ we have

(1) $(\widetilde{v}, \widetilde{A})$ satisfies $(5.22)$ and $\widetilde{\rho} \leqslant 1$,

(2) $\tilde{\rho}=1$ and $\varphi=\widetilde{\varphi}$ in $\Omega \backslash \bigcup B_{i}$,

(3) $\left\|\rho\left(\nabla \varphi-A_{v}\right)-\widetilde{\rho}(\nabla \widetilde{\varphi}-\widetilde{A})\right\|_{L^{2}(\Omega)}^{2}=o(1)$,

(4) $\left\|\operatorname{curl}\left(A_{v}\right)-\operatorname{curl}(\widetilde{A})\right\|_{L^{2}(\Omega)}^{2} \leqslant C|\ln \varepsilon|^{-2}$,

(5) $\mathcal{F}(\widetilde{v}, \widetilde{A}) \leqslant \mathcal{F}\left(v, A_{v}\right)+o(1)$.

Lemma B.1 is proved in [15, Lemma 1] for $\alpha \equiv 1$. The adaptation to our case is presented below.

Proof of Lemma B.1. - The proof of the lemma follows the same lines than in [15].

We define a continuous function $\chi_{\varepsilon}=\chi:[0,1] \rightarrow[0,1]$ by letting

$$
\begin{cases}\chi(x)=x & \text { if } 0 \leqslant x \leqslant 1 / 2 \\ \chi(x)=1 & \text { if } x \geqslant 1-|\ln \varepsilon|^{-2} \\ \chi \text { is affine } & \text { if } 1 / 2 \leqslant x \leqslant 1-|\ln \varepsilon|^{-2} .\end{cases}
$$

We then let $\widetilde{v}:=\frac{\chi(\rho)}{\rho} v \in H^{1}(\Omega, \mathbb{C})$ and we let $\widetilde{A}=A_{\tilde{v}}$ given by Lemma 5.8 . Letting $\widetilde{h}=\operatorname{curl}(\widetilde{A})$ we then get

$$
-\nabla^{\perp} \widetilde{h}=\alpha(\imath \widetilde{v}) \cdot(\nabla \widetilde{v}-\imath \widetilde{A} \widetilde{v}) .
$$

Exactly as in [15] we have

$$
\|v \wedge \nabla v-\widetilde{v} \wedge \nabla \widetilde{v}\|_{L^{2}(\Omega)}^{2} \leqslant C|\ln \varepsilon|^{-2} .
$$

As in [15], from (3.1), (5.22) and (B.2) we obtain PDE of the second order satisfied by $A$ and $\widetilde{A}$.

By considering the difference of these PDE we get

$$
\begin{aligned}
-\Delta(\widetilde{A}-A)+ & \alpha(\widetilde{A}-A) \\
& =\alpha(\widetilde{v} \wedge \nabla \widetilde{v}-v \wedge \nabla v)+\alpha\left(1-\rho^{2}\right) A+\alpha\left(1-\widetilde{\rho}^{2}\right) \widetilde{A} .
\end{aligned}
$$

From (5.3), (B.1) and (B.3), the RHS of (B.4) is bounded in $L^{2}(\Omega)$ by $\frac{C}{|\ln \varepsilon|}$.

Since $(\widetilde{A}-A) \cdot \nu=0$ on $\partial \Omega$, by elliptic regularity, we deduce Assertions 3 and 4 of the lemma.

The end of the proof is exactly as in [15] 
From now on we replace $\left(v, A_{v}\right)$ with $(\widetilde{v}, \widetilde{A})$ and we claim that the valued disks given by Proposition 5.5 is valid for $\left(v, A_{v}\right)$ and $(\widetilde{v}, \widetilde{A})$ and getting the conclusions of Theorem 5.6 for $(\widetilde{v}, \widetilde{A})$ implies the same for $(v, A)$.

In order to simplify the presentation we write $(v, A)$ instead of $(\widetilde{v}, \widetilde{A})$.

\section{B.2. Energetic Decomposition}

We have the following lower bound:

Proposition B.2. - let $h:=\operatorname{curl}(A), h_{0}:=\Delta \xi_{0}=1+\xi_{0}, f:=h-$ $h_{\mathrm{ex}} h_{0}$ and let $\left\{B_{i}=B\left(a_{i}, r_{i}\right) \mid i \in \mathcal{J}\right\}$ be the disks given by Proposition 5.5. We have:

$$
\begin{aligned}
\mathcal{F}(v, A) \geqslant h_{\mathrm{ex}}^{2} \mathbf{J}_{\mathbf{0}}+\sum \mathcal{F}\left[(v, A), B_{i}\right]+2 \pi h_{\mathrm{ex}} \sum d_{i} \xi_{0}\left(a_{i}\right) \\
+\frac{1}{2} \int_{\Omega \backslash \bigcup B_{i}}|\nabla f|^{2}+\frac{1}{2} \int_{\Omega} f^{2}-o(1)
\end{aligned}
$$

where

$$
\mathcal{F}\left[(v, A), B_{i}\right] \geqslant \pi b^{2}\left|d_{i}\right|(|\ln \varepsilon|-C \ln |\ln \varepsilon|) .
$$

This estimate is the starting point of the main argument of [15].

Proof of Proposition B.2. - Let $\widetilde{\Omega}:=\Omega \backslash \bigcup \overline{B_{i}}$. With (B.6) we get

$$
\mathcal{F}\left[(v, A), \cup B_{i}\right] \geqslant \pi b^{2} \sum_{i}\left|d_{i}\right|[|\ln \varepsilon|-C \ln |\ln \varepsilon|] .
$$

On the other hand, letting $f:=h-h_{\mathrm{ex}} h_{0}$ and since $\alpha|\nabla v-\imath A v|^{2} \geqslant|\nabla h|^{2}$, we get

$$
\begin{aligned}
& \frac{1}{2} \int_{\tilde{\Omega}} \alpha|\nabla v-\imath A v|^{2}+\left|h-h_{\mathrm{ex}}\right|^{2} \\
& \quad \geqslant h_{\mathrm{ex}}^{2} \mathbf{J}_{\mathbf{0}}+\frac{1}{2}\|f\|_{H^{1}(\tilde{\Omega})}^{2}+h_{\mathrm{ex}} \int_{\tilde{\Omega}} \nabla f \cdot \nabla\left(h_{0}-1\right)+f\left(h_{0}-1\right)+o(1) .
\end{aligned}
$$

Before refining the above lower bound we make some preliminary claims. We first note that from (B.2) we have $\left\|h-h_{\mathrm{ex}}\right\|_{H^{1}(\Omega)}^{2} \leqslant C\|\nabla v-\imath A v\|_{L^{2}(\Omega)}^{2}=$ $\mathcal{O}\left(h_{\mathrm{ex}}^{2}\right)$. Then $\|f\|_{H^{1}(\Omega)}^{2}=\mathcal{O}\left(h_{\mathrm{ex}}^{2}\right)$. Consequently for $g \in\{f, h\}$ we have

$$
h_{\mathrm{ex}} \int_{\cup B_{i} \cap \Omega}\left|\nabla g \cdot \nabla\left(h_{0}-1\right)\right|+\left|g\left(h_{0}-1\right)\right| \leqslant C\|g\|_{H^{1}(\Omega)} h_{\mathrm{ex}} \sum r_{i}=o(1) \text {. }
$$

We also observe that

$$
\int_{\Omega}-A^{\perp} \cdot \nabla\left(h_{0}-1\right)+h\left(h_{0}-1\right)=0 .
$$


With (5.6) we get $\|A\|_{L^{\infty}(\Omega)} \leqslant C h_{\mathrm{ex}}$ and then (with (B.2))

$$
\begin{aligned}
\sum_{B_{i} \subset \Omega}\left|\int_{\partial B_{i}} \partial_{\tau} \varphi\left(h_{0}-h_{0}\left(a_{i}\right)\right)\right| & =\sum_{B_{i} \subset \Omega}\left|\int_{\partial B_{i}}\left(h_{0}-h_{0}\left(a_{i}\right)\right)\left(\alpha^{-1} \nabla^{\perp} h+A\right) \cdot \tau\right| \\
& \leqslant \sum_{B_{i} \subset \Omega}\left[\left|\int_{\partial B_{i}} \alpha^{-1}\left(h_{0}-h_{0}\left(a_{i}\right)\right) \partial_{\nu} h\right|+C h_{\mathrm{ex}} r_{i}\right] .
\end{aligned}
$$

If $B_{i} \subset \Omega$ we have

$$
\begin{aligned}
& \left|\int_{\partial B_{i}} \alpha^{-1}\left(h_{0}-h_{0}\left(a_{i}\right)\right) \partial_{\nu} h\right| \\
& \quad=\left|\int_{B_{i}} \alpha^{-1} \nabla h_{0} \cdot \nabla h+\left(h_{0}-h_{0}\left(a_{i}\right)\right) \operatorname{div}\left(\alpha^{-1} \nabla h\right)\right| \\
& \quad \leqslant\left|\int_{B_{i}}\left(h_{0}-h_{0}\left(a_{i}\right)\right) \operatorname{div}\left[v \wedge\left(\nabla^{\perp} v-\imath A^{\perp} v\right)\right]\right|+\mathcal{O}\left(h_{\mathrm{ex}} r_{i}\right) \\
& \quad \leqslant \int_{B_{i}}\left|h_{0}-h_{0}\left(a_{i}\right)\right|\left[2\left|\partial_{1} v \wedge \partial_{2} v\right|+4|\nabla(|v|)||A|+|v|^{2}|h|\right]+\mathcal{O}\left(h_{\mathrm{ex}} r_{i}\right) \\
& \quad \leqslant C r_{i} h_{\mathrm{ex}}^{2} .
\end{aligned}
$$

And then

$$
\sum_{B_{i} \subset \Omega}\left|\int_{\partial B_{i}} \partial_{\tau} \varphi\left(h_{0}-h_{0}\left(a_{i}\right)\right)\right| \leqslant C \sum_{B_{i} \subset \Omega} r_{i} h_{\mathrm{ex}}^{2} .
$$

If $B_{i} \not \subset \Omega$, then $\left\|h_{0}-1\right\|_{L^{\infty}\left(B_{i} \cap \Omega\right)} \leqslant C r_{i}$ and

$$
\begin{aligned}
& \left|\int_{\partial\left(B_{i} \cap \Omega\right)}\left(h_{0}-1\right) \partial_{\tau} \varphi\right| \\
& \quad \leqslant \int_{B_{i} \cap \Omega}\left|\nabla h_{0} \cdot \nabla h\right|+\left|h_{0}-1\right|\left[2\left|\partial_{1} v \wedge \partial_{2} v\right|+4|\nabla(|v|)||A|+|v|^{2}|h|\right] \\
& \quad \leqslant C r_{i} h_{\mathrm{ex}}^{2} .
\end{aligned}
$$

By combining (B.9) with (B.10) we deduce:

$$
\sum \int_{\partial B_{i} \cap \Omega}\left(h_{0}-1\right) \partial_{\tau} \varphi=2 \pi \sum d_{i}\left(h_{0}\left(a_{i}\right)-1\right)+o(1) .
$$

We used that if $B_{i} \not \subset \Omega$ then $d_{i}=0$.

We end the preliminary claims by noting that

$$
\int_{\Omega}\left|\alpha^{-1}-1\right|\left|\nabla h \cdot \nabla\left(h_{0}-1\right)\right| \leqslant C h_{\mathrm{ex}}\left\|\alpha^{-1}-1\right\|_{L^{2}(\Omega)}=o\left(h_{\mathrm{ex}}^{-1}\right) .
$$


On the one hand, since $-\Delta f+f=-\Delta h+h$, we have with (B.7), (B.8), (B.11), (B.12) and integrations by parts:

$$
\begin{aligned}
\int_{\tilde{\Omega}} \nabla f \cdot \nabla\left(h_{0}-1\right)+f\left(h_{0}-1\right) & =\int_{\Omega} \alpha^{-1} \nabla h \cdot \nabla\left(h_{0}-1\right)+h\left(h_{0}-1\right)+o\left(h_{\mathrm{ex}}^{-1}\right) \\
& =o\left(h_{\mathrm{ex}}^{-1}\right)+\sum_{i} \int_{\partial B_{i}} \partial_{\tau} \varphi\left(h_{0}-1\right) \\
& =o\left(h_{\mathrm{ex}}^{-1}\right)+2 \pi \sum_{B_{i} \subset \Omega} d_{i}\left[h_{0}\left(a_{i}\right)-1\right] \\
& =o\left(h_{\mathrm{ex}}^{-1}\right)+2 \pi \sum_{B_{i} \subset \Omega} d_{i} \xi_{0}\left(a_{i}\right) .
\end{aligned}
$$

On the other hand, since $\|f\|_{L^{4}(\Omega)} \leqslant C h_{\mathrm{ex}}$, we get $\int_{\cup B_{i}} f^{2}=o\left(h_{\mathrm{ex}}^{-4}\right)$, and this estimate ends the proof.

\section{B.3. Estimate related with the signs of the $d_{i}$ 's}

By Proposition B.2 we have:

$$
\begin{aligned}
0 \geqslant \pi b^{2} \sum_{i}\left|d_{i}\right|(|\ln \varepsilon|-C \ln |\ln \varepsilon|) & +2 \pi h_{\mathrm{ex}} \sum_{i} d_{i} \xi_{0}\left(a_{i}\right) \\
& +\frac{1}{2} \int_{\Omega \backslash B_{i}}|\nabla f|^{2}+\frac{1}{2} \int_{\Omega} f^{2}-o(1) .
\end{aligned}
$$

Denote $I_{+}:=\left\{i \in \mathcal{J} \mid d_{i}>0\right\}, I_{-}:=\left\{i \in \mathcal{J} \mid d_{i}<0\right\}, D:=\sum_{\mathcal{J}}\left|d_{i}\right|$, $D_{+}:=\sum_{i \in I_{+}} d_{i}$ and $D_{-}:=\sum_{i \in I_{-}}\left|d_{i}\right|$.

With (B.13) we obtain $2 h_{\mathrm{ex}} D_{+}\left\|\xi_{0}\right\|_{L^{\infty}(\Omega)} \geqslant b^{2} D|\ln \varepsilon|\left(1-\frac{C \ln |\ln \varepsilon|}{|\ln \varepsilon|}\right)+o(1)$ and then:

$$
D_{-} \leqslant D_{+} \times \frac{C \ln |\ln \varepsilon|}{|\ln \varepsilon|}+o(1) \text {. }
$$

\section{B.4. Estimate related with $\operatorname{dist}\left(a_{i}, \Lambda\right)$}

From Lemma 1.1 , there exist $\eta>0$ and $M \geqslant 1$ s.t., for $a \in \Omega, \xi_{0}(a) \geqslant$ $\min \xi_{0}+\eta \operatorname{dist}(a, \Lambda)^{M}$.

We let $I_{0}:=\left\{\left.i \in I\left|\operatorname{dist}\left(a_{i}, \Lambda\right)<\right| \ln \varepsilon\right|^{-\frac{1}{2 M}}\right\}$ and $D_{0}:=\sum_{i \in I_{0}}\left|d_{i}\right|$. 
If $i \notin I_{0}$, then $\left|\xi_{0}\left(a_{i}\right)\right| \leqslant\left\|\xi_{0}\right\|_{L^{\infty}(\Omega)}-\frac{\eta}{\sqrt{|\ln \varepsilon|}}$. We thus have

$$
\begin{aligned}
\left|\sum_{i} d_{i} \xi_{0}\left(a_{i}\right)\right| & \leqslant\left|\sum_{i \in I_{0}} d_{i} \xi_{0}\left(a_{i}\right)\right|+\left|\sum_{i \notin I_{0}} d_{i} \xi_{0}\left(a_{i}\right)\right| \\
& \leqslant D_{0}\left\|\xi_{0}\right\|_{L^{\infty}(\Omega)}+\left(D-D_{0}\right)\left(\left\|\xi_{0}\right\|_{L^{\infty}(\Omega)}-\frac{\eta}{\sqrt{|\ln \varepsilon|}}\right) \\
& \leqslant D\left\|\xi_{0}\right\|_{L^{\infty}(\Omega)}-\left(D-D_{0}\right) \frac{\eta}{\sqrt{|\ln \varepsilon|}} .
\end{aligned}
$$

From (B.13) we may deduce

$$
2 h_{\mathrm{ex}}\left(D\left\|\xi_{0}\right\|_{L^{\infty}(\Omega)}-\left(D-D_{0}\right) \frac{\eta}{\sqrt{\mid \overline{\ln \varepsilon \mid}}}\right) \geqslant b^{2} D(|\ln \varepsilon|-C \ln |\ln \varepsilon|)-o(1)
$$

and consequently

$$
D-D_{0} \leqslant C D \frac{\ln |\ln \varepsilon|}{\sqrt{|\ln \varepsilon|}}+o(1)
$$

\section{B.5. Estimate of the two last terms in (B.13)}

We let $t \geqslant|\ln \varepsilon|^{-\frac{1}{2 M}} \geqslant|\ln \varepsilon|^{-1 / 2}$ and then $t \geqslant \delta$ since $\delta|\ln \varepsilon|^{1 / 2} \leqslant 1$.

On the one hand, from Lemma E.1 in [6], by denoting $\mathscr{C}_{t}$ a circle with radius $t$ we get:

$$
\int_{\mathscr{C}_{t} \cap \Omega}\left|1-\alpha^{-1}\right| \leqslant C_{b} \lambda t
$$

We assume now that the center of $\mathscr{C}_{t}$ is in $\Lambda$ and $t$ is s.t. $\mathscr{C}_{t} \subset \widetilde{\Omega}=\Omega \backslash \bigcup \overline{B_{i}}$. We denote also $B_{t}$ the disk bounded by $\mathscr{C}_{t}$. On $\mathscr{C}_{t}$ we have $|v|=1$ and then $v=\mathrm{e}^{\imath \varphi}$ with $\varphi$ locally defined.

By direct calculations, we have (with $f=h-h_{\mathrm{ex}} h_{0}, \nu$ the outward normal unit vector to $\mathscr{C}_{t}$ and $\tau=\nu^{\perp}$ ):

$$
\int_{\mathscr{C}_{t}} \alpha^{-1} \partial_{\nu} h=-\int_{\mathscr{C}_{t}}\left[\partial_{\tau} \varphi-A \cdot \tau\right]=-2 \pi d_{t}+\int_{B_{t}} h \text { with } d_{t}:=\operatorname{deg}_{\mathscr{C}_{t}}(v) .
$$

On the other hand $\int_{\mathscr{C}_{t}} \alpha^{-1} \partial_{\nu} h_{0}=\int_{B_{t}} h_{0}+\int_{\mathscr{C}_{t}}\left(\alpha^{-1}-1\right) \partial_{\nu} h_{0}$. Note that

$$
\left|\int_{\mathscr{C}_{t}}\left(\alpha^{-1}-1\right) \partial_{\nu} h_{0}\right| \leqslant\left\|\nabla h_{0}\right\|_{L^{\infty}(\Omega)} \int_{\mathscr{C}_{t}}\left|1-\alpha^{-1}\right| \leqslant C_{b} \lambda t\left\|\nabla h_{0}\right\|_{L^{\infty}(\Omega)} .
$$


Then for $\varepsilon>0$ sufficiently small: $-\int_{\mathscr{C}_{t}} \alpha^{-1} \partial_{\nu} f+\int_{B_{t}} f \geqslant 2 \pi d_{t}-C \lambda h_{\mathrm{ex}} t$. Consequently we obtain

$$
2 \int_{\mathscr{C}_{t}} \alpha^{-2} \int_{\mathscr{C}_{t}}\left|\partial_{\nu} f\right|^{2}+2 \pi t^{2} \int_{B_{t}} f^{2} \geqslant 4 \pi^{2} d_{t}^{2}-C t \lambda h_{\mathrm{ex}}\left|d_{t}\right|
$$

and thus, by denoting $m_{t}:=\int_{\mathscr{C}_{t}} \alpha^{-2}$, we get

$$
\frac{1}{2} \int_{\mathscr{C}_{t}}\left|\partial_{\nu} f\right|^{2}+\frac{\pi t^{2}}{2 m_{t}} \int_{B_{t}} f^{2} \geqslant \frac{\pi^{2} d_{t}^{2}}{m_{t}}-\frac{C t \lambda h_{\mathrm{ex}}\left|d_{t}\right|}{m_{t}} .
$$

Since $2 \pi t \leqslant m_{t} \leqslant b^{-4} 2 \pi t$, for sufficiently $\varepsilon>0$ small we obtain

$$
\frac{1}{2} \int_{\mathscr{C}_{t}}\left|\partial_{\nu} f\right|^{2}+\frac{t}{4} \int_{B_{t}} f^{2} \geqslant b^{4} \frac{\pi d_{t}^{2}}{2 t}-C \lambda h_{\mathrm{ex}}\left|d_{t}\right| \geqslant b^{4} \frac{\pi d_{t}^{2}}{4 t} .
$$

Following exactly the argument in [15] we get

$$
\frac{1}{2} \int_{\Omega \backslash \bigcup B_{i}}|\nabla f|^{2}+\frac{1}{2} \int_{\Omega} f^{2} \geqslant C^{\prime} D^{2} \ln |\ln \varepsilon|+o(1) .
$$

With (B.13) and $\xi_{0}\left(a_{i}\right) \leqslant-\left\|\xi_{0}\right\|_{L^{\infty}(\Omega)}$ there are $C_{1}, C_{2}>0$ (independent of $\varepsilon)$ s.t.

$$
\left(C_{1} D^{2}-C_{2} D\right) \ln |\ln \varepsilon| \leqslant g(\varepsilon) \text { with } g(\varepsilon) \rightarrow 0 \text { for } \varepsilon \rightarrow 0 .
$$

This estimate implies $D \leqslant \frac{C_{1}}{C_{2}}$. Therefore with (B.14) and (B.15) we get the three first assertion of the theorem.

It remains to get (5.15) whose proof follows the same lines as in $[15$, Section 4].

\section{Appendix C. Proof of Proposition 5.7}

Let $C_{0}>1,\left(v_{\varepsilon}\right)_{0<\varepsilon<1} \subset H^{1}(\Omega, \mathbb{C}),\left(h_{\mathrm{ex}}\right)_{0<\varepsilon<1} \subset(0, \infty)$ and $\left(\xi_{\varepsilon}\right)_{0<\varepsilon<1} \subset$ $H_{0}^{1} \cap H^{2} \cap W^{1, \infty}(\Omega, \mathbb{R})$ be s.t. (5.17) and (5.18) hold. For simplicity of the presentation we omit the index $\varepsilon$.

Let $\left\{\left(B\left(a_{i}, r_{i}\right), d_{i}\right) \mid i \in \mathcal{J}\right\}$ be as in the proposition and write $B_{i}:=$ $B\left(a_{i}, r_{i}\right)$.

In this proof the letter " $C$ " stands for a quantity bounded by a power of $C_{0}$ whose value may differ from one line to another.

We let $A=\nabla^{\perp} \xi$ and $\widetilde{\Omega}:=\left\{\begin{array}{ll}\Omega \backslash \bigcup \overline{B_{i}} & \text { if }|v| \ngtr 1 / 2 \text { in } \Omega \\ \Omega & \text { if }|v|>1 / 2 \text { in } \Omega\end{array}\right.$. The heart of the proof consists in estimating the quantity $\int_{\Omega}(v \wedge \nabla v) \cdot A$ in (5.13). 
We first get with the help of (5.17) and (5.18) that if $|v| \ngtr 1 / 2$ in $\Omega$ then $\int_{\cup B_{i}} v \wedge \nabla v \cdot A=o(1)$.

We also claim that, letting $w:=v /|v|$ in $\widetilde{\Omega}: \int_{\tilde{\Omega}}(v \wedge \nabla v-w \wedge \nabla w) \cdot A=o(1)$.

In particular, if $|v|>1 / 2$ in $\Omega$ then we have $\int_{\Omega}(v \wedge \nabla v) \cdot A=o(1)$. We thus assume that $|v| \ngtr 1 / 2$ in $\Omega$.

Then, with an integration by part we get

$$
\begin{aligned}
& -\int_{\Omega} w \wedge \nabla w \cdot A \\
& =-\sum_{B_{i} \subset \Omega}\left\{\xi\left(a_{i}\right) \int_{\partial B_{i}}\left(w \wedge \nabla^{\perp} w\right) \cdot \nu+\int_{\partial B_{i}}\left(\xi-\xi\left(a_{i}\right)\right)\left(w \wedge \nabla^{\perp} w\right) \cdot \nu\right\} \\
& +\sum_{B_{i} \not \subset \Omega} \int_{\partial\left(B_{i} \cap \Omega\right)} \xi\left(w \wedge \nabla^{\perp} w\right) \cdot \nu .
\end{aligned}
$$

For $B_{i} \subset \Omega$ we immediately have:

$$
\int_{\partial B_{i}}\left(w \wedge \nabla^{\perp} w\right) \cdot \nu=-2 \pi d_{i}
$$

We define

$$
u:= \begin{cases}v & \text { in } \widetilde{\Omega} \\ u_{i} & \text { in } B_{i} \cap \Omega\end{cases}
$$

where $u_{i}$ is the harmonic extension of $\operatorname{tr}_{\partial\left(B_{i} \cap \Omega\right)}(v)$ in $B_{i} \cap \Omega$. By the Dirichlet principle we have for all $i$ :

$$
\|\nabla u\|_{L^{2}\left(B_{i} \cap \Omega\right)} \leqslant\|\nabla v\|_{L^{2}\left(B_{i} \cap \Omega\right)}=\mathcal{O}(|\ln \varepsilon|) .
$$

It is easy to check that $\left(w \wedge \nabla^{\perp} w\right) \cdot \nu=|u|^{-2}\left(u \wedge \nabla^{\perp} u\right) \cdot \nu$ on $\bigcup_{i} \partial B_{i}$. For $i \in \mathcal{J}$, we let

$$
f_{i}=\left\{\begin{array}{ll}
\xi-\xi\left(a_{i}\right) & \text { if } B_{i} \subset \Omega \\
\xi & \text { if } B_{i} \not \subset \Omega
\end{array} \in H^{2} \cap W^{1, \infty}\left(B_{i} \cap \Omega\right) .\right.
$$

From (5.18) we get

$$
\left\|\nabla f_{i}\right\|_{L^{\infty}\left(B_{i} \cap \Omega\right)} \leqslant C|\ln \varepsilon| .
$$

Our goal is now to estimate $\int_{\partial\left(B_{i} \cap \Omega\right)} f_{i}\left(w \wedge \nabla^{\perp} w\right) \cdot \nu$. We first consider the case where $i \in \mathcal{J}$ is s.t. $|u| \geqslant 1 / 2$ in $B_{i} \cap \Omega$. In this case we may write in $B_{i}: u=|u| \mathrm{e}^{\imath \phi}$ with $\phi \in H^{1}\left(B_{i}, \mathbb{R}\right),\|\phi\|_{H^{1}\left(B_{i}\right)} \leqslant C|\ln \varepsilon|$. We then have 
with (C.4) and an integration by parts

$$
\begin{aligned}
\left|\int_{\partial\left(B_{i} \cap \Omega\right)} f_{i}\left(w \wedge \nabla^{\perp} w\right) \cdot \nu\right| \\
\leqslant\left\|\nabla f_{i}\right\|_{L^{2}\left(B_{i} \cap \Omega\right)}\|\nabla \phi\|_{L^{2}\left(B_{i} \cap \Omega\right)} \leqslant C|\ln \varepsilon|^{2} r_{i} .
\end{aligned}
$$

We now assume $i \in \mathcal{J}$ is s.t. $|u| \ngtr 1 / 2$ in $B_{i} \cap \Omega$. By smoothness of $\left|u_{i}\right|^{2} \in$ $C^{\infty}\left(B_{i} \cap \Omega, \mathbb{R}\right)$, there exists $\left.t_{i} \in\right] 1 / 5,1 / 4\left[\right.$, a regular value of $\left|u_{i}\right|^{2}$, s.t. $\omega_{i}:=\left\{\left|u_{i}\right|^{2}<t_{i}\right\} \neq \emptyset$. We denote $D_{i}:=\Omega \cap\left[B_{i} \backslash \bar{\omega}_{i}\right]$. Since $|u|^{2} \geqslant 1 / 4$ on $\partial B_{i} \cap \Omega$ we have $\partial D_{i}=\left(\partial B_{i} \cap \Omega\right) \cup \partial \omega_{i} \cup\left(\partial \Omega \cap \overline{D_{i}}\right)$.

Letting $W:=\frac{u}{|u|} \wedge \nabla^{\perp}\left(\frac{u}{|u|}\right)$ we then get

$$
\int_{\partial D_{i}} f_{i} W \cdot \nu=\int_{D_{i}} f_{i} \operatorname{div}(W)+\nabla f_{i} \cdot W .
$$

It is standard to check that $\operatorname{div}(W)=0$ in $D_{i}$. Moreover:

$$
\left|\int_{D_{i}} \nabla f_{i} \cdot W\right| \leqslant 2\|\nabla \xi\|_{L^{2}\left(B_{i} \cap \Omega\right)}\|\nabla u\|_{L^{2}\left(B_{i} \cap \Omega\right)} \leqslant C|\ln \varepsilon|^{2} r_{i} .
$$

Consequently using (C.6) we may deduce

$$
\left|\int_{\partial D_{i}} f_{i} W \cdot \nu\right| \leqslant C|\ln \varepsilon|^{2} r_{i}
$$

On the other hand, from (C.4), $\xi=0$ on $\partial \Omega$ and $\operatorname{div}\left(u \wedge \nabla^{\perp} u\right)=-2 \partial_{1} u \wedge$ $\partial_{2} u$ in $B_{i} \cap \Omega$, we get

$$
\begin{aligned}
& \left|\int_{\partial D_{i}} f_{i} W \cdot \nu-\int_{\partial B_{i} \cap \Omega} f_{i}\left(w \wedge \nabla^{\perp} w\right) \cdot \nu\right| \\
& \quad=\left|\int_{\partial \omega_{i}} f_{i} W \cdot \nu\right| \\
& \quad=\frac{1}{t_{i}}\left|\int_{\omega_{i}}-2 f_{i} \partial_{1} u \wedge \partial_{2} u+\nabla f_{i} \cdot\left(u \wedge \nabla^{\perp} u\right)\right| \leqslant C|\ln \varepsilon|^{3} r_{i} .
\end{aligned}
$$

We may conclude by using (C.1), (C.2), (C.5), (C.7) and (C.8):

$$
-\int_{\Omega} v \wedge \nabla v \cdot A=2 \pi \sum_{B_{i} \subset \Omega} d_{i} \xi\left(a_{i}\right)+o(1) .
$$

The rest of the proof is exactly the same than in [17]. 


\section{Appendix D. Proof of some results of Section 6.1.1}

\section{D.1. Proof of Proposition 6.4}

We use the same notation than in Proposition 6.4. In this proof, the letter $C$ is a quantity which depends only on $\Omega, N$ and $\sum_{i}\left|d_{i}\right|$, its value may change from one line to another.

We argue as in [13]. We let $\Phi_{\star}^{(\mathbf{z}, \mathbf{d})} \in \bigcap_{0<p<2} W^{1, p}(\Omega, \mathbb{R}) \cap H_{\text {loc }}^{1}(\Omega \backslash$ $\left.\left\{z_{1}, \ldots, z_{N}\right\}, \mathbb{R}\right)$ be the unique solution of

$$
\begin{cases}\Delta \Phi_{\star}^{(\mathbf{z}, \mathbf{d})}=2 \pi \sum_{i=1}^{N} d_{i} \delta_{z_{i}} & \text { in } \Omega \\ \Phi_{\star}^{(\mathbf{z}, \mathbf{d})}=0 & \text { on } \partial \Omega\end{cases}
$$

and let $\Phi_{\tilde{r}} \in H^{1}\left(\Omega_{\tilde{r}}, \mathbb{R}\right)$ be the unique solution of

$$
\begin{cases}\Delta \Phi_{\tilde{r}}=0 & \text { in } \Omega_{\tilde{r}} \\ \Phi_{\tilde{r}}=0 & \text { on } \partial \Omega \\ \Phi_{\tilde{r}}=\text { Cst }_{i} & \text { on } \partial B\left(z_{i}, \widetilde{r}\right) \\ \int_{\partial B\left(z_{i}, \tilde{r}\right)} \partial_{\nu} \Phi_{\tilde{r}}=2 \pi d_{i} & \text { for all } i \in\{1, \ldots, N\} .\end{cases}
$$

We then have $\nabla^{\perp} \Phi_{\star}^{(\mathbf{z}, \mathbf{d})}=w_{\star}^{(\mathbf{z}, \mathbf{d})} \wedge \nabla w_{\star}^{(\mathbf{z}, \mathbf{d})}$ and $\nabla^{\perp} \Phi_{\tilde{r}}^{(\mathbf{z}, \mathbf{d})}=w_{\tilde{r}}^{(\mathbf{z}, \mathbf{d})} \wedge \nabla w_{\tilde{r}}^{(\mathbf{z}, \mathbf{d})}$. It is important to note that if $w \in H^{1}\left(\Omega_{\tilde{r}}, \mathbb{S}^{1}\right)$, then $|\nabla w|=|w \wedge \nabla w|$.

We may decompose $\Phi_{\star}^{(\mathbf{z}, \mathbf{d})}$ as $\Phi_{\star}^{(\mathbf{z}, \mathbf{d})}=\sum_{i} d_{i} \Phi_{z_{i}}$ where, for $z \in \Omega, \Phi_{z}$ is the unique solution of

$$
\begin{cases}\Delta \Phi_{z}=2 \pi \delta_{z} & \text { in } \Omega \\ \Phi_{z}=0 & \text { on } \partial \Omega .\end{cases}
$$

With a standard pointwise bound for the gradient of an harmonic function (see in $[10,(2.31)])$ we have $\left\|\nabla \Phi_{z_{i}}\right\|_{L^{\infty}\left(\Omega \backslash \overline{B\left(z_{i}, \tilde{r}\right)}\right)} \leqslant C \frac{\left\|\Phi_{z_{i}}\right\|_{L^{\infty}\left(\Omega \backslash \overline{B\left(z_{i}, \tilde{r} / 4\right)}\right)}}{\tilde{r}}$. Thus

$$
\left\|\nabla \Phi_{\star}^{(\mathbf{z}, \mathbf{d})}\right\|_{L^{\infty}\left(\Omega_{\tilde{r}}\right)} \leqslant C \frac{\sum_{i}\left|d_{i}\right|\left\|\Phi_{z_{i}}\right\|_{L^{\infty}\left(\Omega_{\tilde{r} / 4}\right)}}{\widetilde{r}} .
$$

Moreover, it is easy to check that $\Phi_{z_{i}}=\ln \left|x-z_{i}\right|+R_{z_{i}}$ where $R_{z_{i}}$ is the harmonic extension of $-\ln \left|x-z_{i}\right|_{\mid \partial \Omega}$. From (D.2) and by the maximum principle we get for $\widetilde{r}<\min \left\{[\operatorname{diam}(\Omega)]^{-1} ; 1 / 4\right\}$

$$
\left|\nabla \Phi_{\star}^{(\mathbf{z}, \mathbf{d})}\right| \leqslant \frac{C(1+|\ln \widetilde{r}|)}{\widetilde{r}} \text { in } \Omega_{\tilde{r}}
$$

which proves (6.7). 
If there is $\eta>0$ s.t. $\hbar>\eta$, then $\left\|R_{z_{i}}\right\|_{C^{1}(\Omega)} \leqslant C_{\eta}$ where $C_{\eta}$ which depends only on $\eta$ and $\Omega$. We thus get $\left\|\nabla \Phi_{\star}^{(\mathbf{z}, \mathbf{d})}\right\|_{L^{\infty}\left(\Omega_{\tilde{r}}\right)} \leqslant \frac{\tilde{C}_{\eta}}{\tilde{r}}$ (where $\widetilde{C}_{\eta}$ depends only on $\eta, N, \sum\left|d_{i}\right|$ and $\Omega$ ) and this estimates implies (6.11).

We now define $R_{(\mathbf{z}, \mathbf{d})}:=\sum_{i} d_{i} R_{z_{i}}$ in order to have $\Phi_{\star}^{(\mathbf{z}, \mathbf{d})}=\sum_{i} d_{i} \ln \mid x-$ $z_{i} \mid+R_{(\mathbf{z}, \mathbf{d})}$.

From Lemma I.4 in [4] we have

$$
\begin{aligned}
\left\|\Phi_{\tilde{r}}-\Phi_{\star}^{(\mathbf{z}, \mathbf{d})}\right\|_{L^{\infty}\left(\Omega_{\tilde{r}}\right)} \leqslant \sum_{i} & {\left[\sup _{\partial B\left(z_{i}, \tilde{r}\right)} \sum_{j} \ln \left|x-z_{j}\right|-\inf _{\partial B\left(z_{i}, \tilde{r}\right)} \sum_{j} \ln \left|x-z_{j}\right|\right] } \\
& \left.+\sum_{i}\left[\sup _{\partial B\left(z_{i}, \tilde{r}\right)} R_{(\mathbf{z}, \mathbf{d})}-\inf _{\partial B\left(z_{i}, \tilde{r}\right)} R_{(\mathbf{z}, \mathbf{d})}\right] . \quad \text { (D. } 4\right)
\end{aligned}
$$

If $N=1$, then the first term of the RHS in (D.4) is 0. Otherwise, as in [17, Proposition 5.1], we have

$$
\sum_{i}\left[\sup _{\partial B\left(z_{i}, \tilde{r}\right)} \sum_{j} \ln \left|x-z_{j}\right|-\inf _{\partial B\left(z_{i}, \tilde{r}\right)} \sum_{j} \ln \left|x-z_{j}\right|\right] \leqslant \frac{C \widetilde{r}}{\min _{i \neq j}\left|z_{i}-z_{j}\right|} .
$$

And for $i \in\{1, \ldots, N\}$, by harmonicity of $R_{(\mathbf{z}, \mathbf{d})}$, for $0<\rho<\frac{\hbar}{2}$ we get

$$
\left\|\nabla R_{(\mathbf{z}, \mathbf{d})}\right\|_{L^{\infty}\left(B\left(z_{i}, \rho\right)\right)} \leqslant \frac{C\left\|R_{(\mathbf{z}, \mathbf{d})}\right\|_{L^{\infty}(\Omega)}}{\operatorname{dist}\left(z_{i}, \partial \Omega\right)-\rho} \leqslant C \frac{1+|\ln (\hbar)|}{\hbar} .
$$

Then

$$
\sum_{i}\left[\sup _{\partial B\left(z_{i}, \tilde{r}\right)} R_{(\mathbf{z}, \mathbf{d})}-\inf _{\partial B\left(z_{i}, \tilde{r}\right)} R_{(\mathbf{z}, \mathbf{d})}\right] \leqslant C \frac{\widetilde{r}(1+|\ln (\hbar)|)}{\hbar} .
$$

We let

$$
Y:= \begin{cases}\frac{\tilde{r}(1+|\ln (\hbar)|)}{\hbar} & \text { if } N=1 \\ \frac{\tilde{r}}{\min _{i \neq j}\left|z_{i}-z_{j}\right|}+\frac{\tilde{r}(1+|\ln (\hbar)|)}{\hbar} & \text { if } N \geqslant 2 .\end{cases}
$$

By combining (D.4), (D.5) and (D.7) we get

$$
\left\|\Phi_{\tilde{r}}-\Phi_{\star}^{(\mathbf{z}, \mathbf{d})}\right\|_{L^{\infty}\left(\Omega_{\tilde{r}}\right)} \leqslant C Y .
$$

From (D.3) and (D.9) we immediately get

$$
\begin{aligned}
0 & \leqslant \int_{\Omega_{\tilde{r}}}\left|\nabla \Phi_{\star}^{(\mathbf{z}, \mathbf{d})}\right|^{2}-\left|\nabla \Phi_{\tilde{r}}\right|^{2}+\left|\nabla\left(\Phi_{\star}^{(\mathbf{z}, \mathbf{d})}-\Phi_{\tilde{r}}\right)\right|^{2} \\
& \leqslant C Y \widetilde{r} \max _{i}\left\|\partial_{\nu} \Phi_{\star}^{(\mathbf{z}, \mathbf{d})}\right\|_{L^{\infty}\left(\partial B\left(z_{i}, \tilde{r}\right)\right)} .
\end{aligned}
$$

On the other hand, for $i \in\{1, \ldots, N\}$, we have (with (D.6))

$$
\left\|\partial_{\nu} \Phi_{\star}^{(\mathbf{z}, \mathbf{d})}\right\|_{L^{\infty}\left(B\left(z_{i}, \tilde{r}\right)\right)} \leqslant C\left(\frac{1}{\widetilde{r}}+\frac{1+|\ln (\hbar)|}{\hbar}\right) .
$$


Using $X$ defined in (6.8), from (D.10) and (D.11), we get

$$
0 \leqslant \int_{\Omega_{\tilde{r}}}\left|\nabla \Phi_{\star}^{(\mathbf{z}, \mathbf{d})}\right|^{2}-\left|\nabla \Phi_{\tilde{r}}\right|^{2}+\left|\nabla\left(\Phi_{\star}^{(\mathbf{z}, \mathbf{d})}-\Phi_{\tilde{r}}\right)\right|^{2} \leqslant C X .
$$

From (D.12) we deduce (6.10) and since $\int_{\partial \Omega}\left(\varphi_{\star}-\varphi_{\tilde{r}}\right)=0$, with a Poincaré inequality we obtain (6.9).

\section{D.2. Proof of Proposition 6.5}

Let $(\mathbf{z}, \mathbf{d})=(\mathbf{z}, \mathbf{d})^{(n)} \in\left(\Omega^{N}\right)^{*} \times \mathbb{Z}^{N}$ and denote $\hbar:=\min _{i} \operatorname{dist}\left(z_{i}, \partial \Omega\right)>$ 0 . Assume that $d_{1}, \ldots, d_{N}$ are independent of $n$. Let $\widetilde{r}=\widetilde{r}_{n} \rightarrow 0$ be s.t (6.1) holds.

In this proof the letter $C$ stands for a quantity which depends only on $\Omega$, $N, C_{1}$ and $\sum_{i}\left|d_{i}\right|$, its value may change from one line to another.

By Remark 6.3 and an integration by parts we have

$$
\frac{1}{2} \int_{\Omega_{\tilde{r}}}\left|\nabla w_{\star}^{(\mathbf{z}, \mathbf{d})}\right|^{2}=\frac{1}{2} \int_{\Omega_{\tilde{r}}}\left|\nabla \Phi_{\star}^{(\mathbf{z}, \mathbf{d})}\right|^{2}=-\frac{1}{2} \sum_{i} \int_{\partial B\left(z_{i}, \tilde{r}\right)} \Phi_{\star}^{(\mathbf{z}, \mathbf{d})} \partial_{\nu} \Phi_{\star}^{(\mathbf{z}, \mathbf{d})} .
$$

For $i_{0} \in\{1, \ldots, N\}$, we fix $x_{i_{0}} \in \partial B\left(z_{i_{0}}, \widetilde{r}\right)$. Then (with $\nabla^{\perp} \Phi_{\star}^{(\mathbf{z}, \mathbf{d})}=w_{\star}^{(\mathbf{z}, \mathbf{d})} \wedge$ $\left.\nabla w_{\star}^{(\mathbf{z}, \mathbf{d})}\right)$

$$
\begin{aligned}
& \int_{\partial B\left(z_{i_{0}}, \tilde{r}\right)} \Phi_{\star}^{(\mathbf{z}, \mathbf{d})} \partial_{\nu} \Phi_{\star}^{(\mathbf{z}, \mathbf{d})} \\
& \quad=\int_{\partial B\left(z_{i_{0}}, \tilde{r}\right)}\left[\Phi_{\star}^{(\mathbf{z}, \mathbf{d})}-\Phi_{\star}^{(\mathbf{z}, \mathbf{d})}\left(x_{i_{0}}\right)\right] \partial_{\nu} \Phi_{\star}^{(\mathbf{z}, \mathbf{d})}+2 \pi d_{i_{0}} \Phi_{\star}^{(\mathbf{z}, \mathbf{d})}\left(x_{i_{0}}\right) .
\end{aligned}
$$

On the one hand, arguing as in the proof of (D.9), we get for $z \in \partial B\left(z_{i_{0}}, \widetilde{r}\right)$ :

$$
\left|\Phi_{\star}^{(\mathbf{z}, \mathbf{d})}(z)-\Phi_{\star}^{(\mathbf{z}, \mathbf{d})}\left(x_{i_{0}}\right)\right| \leqslant \sup _{\partial B\left(z_{i_{0}}, \tilde{r}\right)} \Phi_{\star}^{(\mathbf{z}, \mathbf{d})}-\inf _{\partial B\left(z_{i_{0}}, \tilde{r}\right)} \Phi_{\star}^{(\mathbf{z}, \mathbf{d})} \leqslant C Y .
$$

Then, using (D.11), we obtain

$$
\sum_{i}\left|\int_{\partial B\left(z_{i}, \tilde{r}\right)}\left[\Phi_{\star}^{(\mathbf{z}, \mathbf{d})}-\Phi_{\star}^{(\mathbf{z}, \mathbf{d})}\left(x_{i}\right)\right] \partial_{\nu} \Phi_{\star}^{(\mathbf{z}, \mathbf{d})}\right| \leqslant C X .
$$

On the other hand, for $i_{0} \in\{1, \ldots, N\}$

$$
\begin{aligned}
\Phi_{\star}^{(\mathbf{z}, \mathbf{d})}\left(x_{i_{0}}\right) & -R_{(\mathbf{z}, \mathbf{d})}\left(z_{i_{0}}\right) \\
= & -d_{i_{0}}|\ln \widetilde{r}|+\sum_{j \neq i_{0}} d_{j} \ln \left|x_{i_{0}}-z_{j}\right|+\left[R_{(\mathbf{z}, \mathbf{d})}\left(x_{i_{0}}\right)-R_{(\mathbf{z}, \mathbf{d})}\left(z_{i_{0}}\right)\right],
\end{aligned}
$$


and with (D.6) we get $\left|R_{(\mathbf{z}, \mathbf{d})}\left(x_{i_{0}}\right)-R_{(\mathbf{z}, \mathbf{d})}\left(z_{i_{0}}\right)\right| \leqslant \frac{C(1+|\ln \hbar|) \tilde{r}}{\hbar}$. We then immediately get:

$$
\Phi_{\star}^{(\mathbf{z}, \mathbf{d})}\left(x_{i_{0}}\right)=R_{(\mathbf{z}, \mathbf{d})}\left(z_{i_{0}}\right)-d_{i_{0}}|\ln \widetilde{r}|+\sum_{j \neq i_{0}} d_{j} \ln \left|z_{i_{0}}-z_{j}\right|+\mathcal{O}(X) .
$$

With (D.14), (D.15) and (D.16) we may prove that (D.13) may be rewritten into

$$
\begin{aligned}
& \frac{1}{2} \int_{\Omega_{\tilde{r}}}\left|\nabla w_{\star}^{(\mathbf{z}, \mathbf{d})}\right|^{2} \\
& \quad=\pi \sum_{i}\left[d_{i}^{2}|\ln \widetilde{r}|-d_{i} R_{(\mathbf{z}, \mathbf{d})}\left(z_{i}\right)\right]-\pi \sum_{j \neq i} d_{j} d_{j} \ln \left|z_{i}-z_{j}\right|+\mathcal{O}(X)
\end{aligned}
$$

where " $\mathcal{O}(X)$ " is quantity bounded by $C X$ with $C$ depending only on $N, \Omega$ and $\sum\left|d_{i}\right|$.

\section{D.3. Proof of Proposition 6.7}

Let $(\mathbf{z}, \mathbf{d})=(\mathbf{z}, \mathbf{d})^{(n)} \in\left(\Omega^{N}\right)^{*} \times \mathbb{Z}^{N}, \widetilde{r} \downarrow 0$ and $\eta>0$ be as in the proposition.

In this proof the letter $C$ stands for a quantity which depends only on $\Omega$, $N$ and $\sum_{i}\left|d_{i}\right|$, its value may change from one line to another.

We first claim that, for $i \neq j, B\left(z_{i}, \eta\right) \cap B\left(z_{j}, \eta\right) \neq \emptyset, B\left(z_{i}, \eta\right) \subset \Omega$ and $\eta=\chi \widetilde{r}$ with $\chi \rightarrow \infty$. In particular we assume $n$ sufficiently large to have $\eta>\widetilde{r}$.

Since $\nabla^{\perp} \Phi_{\star}^{(\mathbf{z}, \mathbf{d})}=w_{\star}^{(\mathbf{z}, \mathbf{d})} \wedge \nabla w_{\star}^{(\mathbf{z}, \mathbf{d})}$, for $i_{0} \in\{1, \ldots, N\}$ and $z \in \Omega \backslash$ $\left\{z_{1}, \ldots, z_{N}\right\}$, we have

$w_{\star}^{(\mathbf{z}, \mathbf{d})} \wedge \nabla w_{\star}^{(\mathbf{z}, \mathbf{d})}(z)=d_{i_{0}} \nabla^{\perp}\left(\ln \left|z-z_{i_{0}}\right|\right)+\nabla^{\perp}\left[R_{(\mathbf{z}, \mathbf{d})}(z)+\sum_{j \neq i_{0}} d_{j} \ln \left|z-z_{j}\right|\right]$.

For $j \in\{1, \ldots, N\}$, let $\theta_{j}$ be the main determination of the argument of $\frac{z-z_{j}}{\left|z-z_{j}\right|}$ and let $\mathcal{R}$ be an harmonic conjugate of $R_{(\mathbf{z}, \mathbf{d})}$. In $\Omega \backslash\left\{z_{1}, \ldots, z_{N}\right\}$ we have

$$
w_{\star}^{(\mathbf{z}, \mathbf{d})} \wedge \nabla w_{\star}^{(\mathbf{z}, \mathbf{d})}-d_{i_{0}} \nabla \theta_{i_{0}}=\nabla\left[\sum_{j \neq i_{0}} d_{j} \theta_{j}+\mathcal{R}\right] .
$$

Then for $z \in B\left(z_{i_{0}}, \eta\right) \backslash\left\{z_{i_{0}}\right\}$ we have $w_{\star}^{(\mathbf{z}, \mathbf{d})}(z)=\left(\frac{z-z_{i_{0}}}{\left|z-z_{i_{0}}\right|}\right)^{d_{i_{0}}} \mathrm{e}^{\imath \varphi_{i_{0}}(z)}$ with $\varphi_{i_{0}}=\sum_{j \neq i_{0}} d_{j} \widetilde{\theta}_{j}+\mathcal{R}+$ Cst $_{i_{0}}$ where, for $j \neq i_{0}, \widetilde{\theta}_{j}$ is a determination 
of the argument of $\frac{z-z_{i}}{\left|z-z_{i}\right|}$ which is globally defined in $B\left(z_{i_{0}}, \eta\right)$. Note that $\varphi_{i_{0}} \in H^{1}\left(B\left(z_{i_{0}}, \eta\right), \mathbb{R}\right)$.

On the other hand, by direct calculations, we have

$$
\left\|\sum_{j \neq i_{0}} d_{j} \nabla \tilde{\theta}_{j}\right\|_{L^{\infty}\left(B\left(z_{i_{0}}, \eta\right)\right)} \leqslant \frac{C}{\eta}
$$

and, since $R_{(\mathbf{z}, \mathbf{d})}$ is harmonic, we also have from the definition of $\mathcal{R}$

$$
\begin{aligned}
\|\nabla \mathcal{R}\|_{L^{\infty}\left(B\left(z_{i_{0}}, \eta\right)\right)}=\left\|\nabla R_{(\mathbf{z}, \mathbf{d})}\right\|_{L^{\infty}\left(B\left(z_{i_{0}}, \eta\right)\right)} & \\
& \leqslant C \frac{\left\|R_{(\mathbf{z}, \mathbf{d})}\right\|_{L^{\infty}(\Omega)}}{\operatorname{dist}\left(B\left(z_{i_{0}}, \eta\right), \partial \Omega\right)} \leqslant C \frac{|\ln (\hbar)|+1}{\hbar} .
\end{aligned}
$$

We thus deduce

$$
\left\|\nabla \varphi_{i_{0}}\right\|_{L^{\infty}\left(B\left(z_{i_{0}}, \eta\right)\right)} \leqslant C\left(\frac{1+|\ln (\hbar)|}{\hbar}+\frac{1}{\eta}\right) .
$$

We switch to polar coordinates by letting for $i \in\{1, \ldots, N\}$ and $\rho \in] \widetilde{r}, \eta[$, $\widetilde{\varphi}_{i}(\rho, \theta):=\varphi_{i}\left(z_{i}+\rho \mathrm{e}^{\imath \theta}\right)$. We then get, by (D.17) and a mean value argument, the existence of $\left.\rho_{n} \in\right] \sqrt{\chi} \widetilde{r}, \eta[$ s.t.

$$
\sum_{i} \int_{0}^{2 \pi}\left|\partial_{\theta} \widetilde{\varphi}_{i}\left(\rho_{n}, \theta\right)\right|^{2} \mathrm{~d} \theta \leqslant \frac{C}{\ln \chi}\left[\frac{\eta(|\ln (\hbar)|+1)}{\hbar}+1\right]^{2} .
$$

We let $Z:=\frac{1}{\ln \chi}\left[\frac{\eta(|\ln (\hbar)|+1)}{\hbar}+1\right]^{2}$ and by assumption we have $Z \rightarrow 0$.

We denote, for $i \in\{1, \ldots, N\}, m_{i}=\frac{1}{2 \pi} \int_{0}^{2 \pi} \widetilde{\varphi}_{i}\left(\rho_{n}, \theta\right) \mathrm{d} \theta$ in order to have

$$
\int_{0}^{2 \pi}\left|\widetilde{\varphi}_{i}\left(\rho_{n}, \theta\right)-m_{i}\right|^{2} \mathrm{~d} \theta \leqslant C Z .
$$

We then define $\phi_{i} \in H^{1}\left(B\left(z_{i}, \rho_{n}\right) \backslash \overline{B\left(z_{i}, \widetilde{r}\right)}, \mathbb{R}\right)$ using polar coordinates:

$$
\widetilde{\phi}_{i}(s, \theta)=\frac{s-\rho_{n}}{\widetilde{r}-\rho_{n}} m_{i}+\frac{s-\widetilde{r}}{\rho_{n}-\widetilde{r}} \widetilde{\varphi}\left(\rho_{n}, \theta\right) \text { with } s \in\left(\widetilde{r}, \rho_{n}\right) .
$$

For $z_{i}+s \mathrm{e}^{\imath \theta} \in B\left(z_{i}, \rho_{n}\right) \backslash \overline{B\left(z_{i}, \widetilde{r}\right)}$, we let $\phi_{i}\left(z_{i}+s \mathrm{e}^{\imath \theta}\right):=\widetilde{\phi}_{i}(s, \theta)$. By standard calculations we get $\int_{B\left(z_{i}, \rho_{n}\right) \backslash \overline{B\left(z_{i}, \tilde{r}\right)}}\left|\nabla \phi_{i}\right|^{2} \leqslant C Z$.

We conclude by defining

$$
v=\left\{\begin{array}{ll}
w_{\star}^{(\mathbf{z}, \mathbf{d})} & \text { in } \Omega \backslash \bigcup \overline{B\left(z_{i}, \rho_{n}\right)} \\
u_{i} \mathrm{e}^{\imath \phi_{i}} & \text { in } B\left(z_{i}, \rho_{n}\right) \backslash \overline{B\left(z_{i}, \widetilde{r}\right)}
\end{array} \text { with } u_{i}(z)=\left(\frac{z-z_{i}}{\left|z-z_{i}\right|}\right)^{d_{i}} .\right.
$$

It is clear that $v \in H^{1}\left(\Omega_{\tilde{r}}, \mathbb{S}^{1}\right)$ and that for $i \in\{1, \ldots, N\}$ we have $v\left(z_{i}+\right.$ $\left.\widetilde{r} \mathrm{e}^{\imath \theta}\right)=\operatorname{Cst}_{i} u_{i}\left(\right.$ with $\left.\operatorname{Cst}_{i}=\mathrm{e}^{\imath m_{i}}\right)$. Note that since $\operatorname{deg}_{\partial B\left(z_{i}, \tilde{r}\right)}\left(w_{\star}^{(\mathbf{z}, \mathbf{d})}\right)=d_{i}$ 
we have

$$
\frac{1}{2} \int_{B\left(z_{i}, \rho_{n}\right) \backslash B\left(z_{i}, \tilde{r}\right)}\left|\nabla u_{i}\right|^{2} \leqslant \frac{1}{2} \int_{B\left(z_{i}, \rho_{n}\right) \backslash B\left(z_{i}, \tilde{r}\right)}\left|\nabla w_{\star}^{(\mathbf{z}, \mathbf{d})}\right|^{2}
$$

and

$$
\begin{aligned}
\frac{1}{2} \int_{B\left(z_{i}, \rho_{n}\right) \backslash B\left(z_{i}, \tilde{r}\right)} & \left|\nabla\left(u_{i} \mathrm{e}^{\imath \phi_{i}}\right)\right|^{2} \\
= & \frac{1}{2} \int_{B\left(z_{i}, \rho_{n}\right) \backslash B\left(z_{i}, \tilde{r}\right)}\left|\nabla u_{i}\right|^{2}+\frac{1}{2} \int_{B\left(z_{i}, \rho_{n}\right) \backslash B\left(z_{i}, \tilde{r}\right)}\left|\nabla \phi_{i}\right|^{2} .
\end{aligned}
$$

Consequently using (D.17) and $\rho_{n}<\eta$ we obtain

$$
\sum_{i} \frac{1}{2} \int_{B\left(z_{i}, \rho_{n}\right) \backslash B\left(z_{i}, \tilde{r}\right)}|\nabla v|^{2} \leqslant \sum_{i} \frac{1}{2} \int_{B\left(z_{i}, \rho_{n}\right) \backslash B\left(z_{i}, \tilde{r}\right)}\left|\nabla w_{\star}^{(\mathbf{z}, \mathbf{d})}\right|^{2}+C Z .
$$

Thus $\frac{1}{2} \int_{\Omega_{\tilde{r}}}|\nabla v|^{2} \leqslant \frac{1}{2} \int_{\Omega_{\tilde{r}}}\left|\nabla w_{\star}^{(\mathbf{z}, \mathbf{d})}\right|^{2}+C Z$. The last estimate and (6.10) end the proof.

\section{Appendix E. Proof of Proposition 7.3}

\section{Proof.}

Step 1. Selection of "good" points. - Let $d \in \mathbb{N}^{*}$ and consider $\mathbf{D} \in \Lambda_{d}$ which minimizes (7.2).

For $k \in\left\{1, \ldots, N_{0}\right\}$, if $D_{k} \geqslant 1$ we let $\left(\widetilde{z}_{1}^{(k)}, \ldots, \widetilde{z}_{D_{k}}^{(k)}\right) \in\left[B\left(p_{k}, h_{\mathrm{ex}}^{-1 / 4}\right)^{D_{k}}\right]^{*}$ which minimizes the infimum in the left hand side of (6.15) with $R=h_{\mathrm{ex}}^{-1 / 4}$, $p=p_{k}$ and $D=D_{k}$.

We then have the existence of $C$ (depending only on $\Omega$ and $d$ ) s.t. $\mid p_{k}-$ $\widetilde{z}_{i}^{(k)} \mid \leqslant C h_{\mathrm{ex}}^{-1 / 2}$ and if $D_{k} \geqslant 2$ then $\left|\widetilde{z}_{i}^{(k)}-\widetilde{z}_{j}^{(k)}\right| \geqslant h_{\mathrm{ex}}^{-1 / 2} / C$ for $i \neq j$.

We may choose (in an arbitrary way) $z_{i}^{(k)} \in B\left(\widetilde{z}_{i}^{(k)}, \delta\right) \cap[\delta(\mathbb{Z} \times \mathbb{Z})]$. Since $\delta \sqrt{h}_{\mathrm{ex}} \rightarrow 0$, we still have (up to changing the value $C$ ) $\left|p_{k}-z_{i}^{(k)}\right| \leqslant C h_{\mathrm{ex}}^{-1 / 2}$ and if $D_{k} \geqslant 2$ then $\left|z_{i}^{(k)}-\widetilde{z}_{j}^{(k)}\right| \geqslant h_{\mathrm{ex}}^{-1 / 2} / C$ for $i \neq j$.

For $i \in\left\{1, \ldots, D_{k}\right\}$ we let $x_{i}^{(k)}:=z_{i}^{(k)}+\lambda \delta x_{0}$ where $x_{0} \in \omega$ is an arbitrary point of minimum of $W^{\text {micro }}$ (defined in (6.21)). 
Step 2. Construction of the test function. - We construct test functions in subdomains of $\Omega$ and then we glue the test functions.

- We let $w_{h_{\mathrm{ex}}}^{\text {macro }} \in H^{1}\left(\Omega_{h_{\mathrm{ex}}^{-1}}(\mathbf{z}), \mathbb{S}^{1}\right)$ be a minimizer of $I_{h_{\mathrm{ex}}^{-1}}^{\mathrm{Dir}}(\mathbf{z}, \mathbf{d})$ (defined in (6.3)) with $\mathbf{D}=(1, \ldots, 1) \in \mathbb{Z}^{d}$ and $\mathbf{z} \in\left(\Omega^{d}\right)^{*}$ is a $d$ tuple s.t. $\left\{z_{1}, \ldots, z_{d}\right\}=\left\{z_{i}^{(k)} \mid k \in\left\{1, \ldots, N_{0}\right\}\right.$ s.t. $D_{k} \geqslant 1$ and $i \in$ $\left.\left\{1, \ldots, D_{k}\right\}\right\}$.

- For $k \in\left\{1, \ldots, N_{0}\right\}$ s.t. $D_{k} \geqslant 1$ and $i \in\left\{1, \ldots, D_{k}\right\}$, we let $w_{k, i}^{\text {micro }} \in$ $H^{1}\left[B\left(z_{i}^{(k)}, h_{\mathrm{ex}}^{-1}\right) \backslash \overline{B\left(x_{i}^{(k)}, \lambda \delta^{2}\right)}, \mathbb{S}^{1}\right]$ be a minimizer of the right hand side of (6.18) with $z_{\varepsilon}=z_{i}^{(k)}, x_{\varepsilon}=x_{i}^{(k)}, R=h_{\mathrm{ex}}^{-1}$ and $r=\lambda \delta^{2}$ (from (7.3) we have $R / r \rightarrow \infty$ ).

We let also $u_{k, i} \in H^{1}\left[B\left(x_{i}^{(k)}, \lambda \delta^{2}\right), \mathbb{C}\right]$ be a minimizer of

$$
u \longmapsto \frac{1}{2} \int_{B\left(x_{i}^{(k)}, \lambda \delta^{2}\right)}|\nabla u|^{2}+\frac{1}{2 \varepsilon^{2}}\left(1-|u|^{2}\right)^{2}
$$

with the Dirichlet boundary condition $u\left(x_{i}^{(k)}+\lambda \delta^{2} \mathrm{e}^{\imath \theta}\right)=\mathrm{e}^{\imath \theta}$.

By considering well chosen constants $\mathrm{Cst}_{k, i}^{(1)}$, $\mathrm{Cst}_{k, i}^{(2)}$ and $\mathrm{Cst}_{k}$, we may glue the above test functions and we define $v \in H^{1}(\Omega, \mathbb{C})$ :

$$
v= \begin{cases}w_{h_{\mathrm{ex}}}^{\text {macro }} & \text { in } \Omega_{h_{\mathrm{ex}}^{-1}(\mathbf{z})} \\ \mathrm{Cst}_{k} & \text { in } B\left(z_{i}^{(k)}, h_{\mathrm{ex}}^{-1}\right) \text { if } D_{k}=0 \\ \mathrm{Cst}_{k, i}^{(1)} w_{k, i}^{\text {micro }} & \text { in } B\left(z_{i}^{(k)}, h_{\mathrm{ex}}^{-1}\right) \backslash B\left(x_{i}^{(k)}, \lambda \delta^{2}\right), \\ \mathrm{Cst}_{k, i}^{(2)} u_{k, i} & k \in\left\{1, \ldots, N_{0}\right\} \text { s.t. } D_{k} \geqslant 1 \text { and } i \in\left\{1, \ldots, D_{k}\right\} \\ & k \in\left\{1, \ldots, x_{0}^{(k)}, \lambda \delta^{2}\right) \\ & \text { in s.t. } D_{k} \geqslant 1 \text { and } i \in\left\{1, \ldots, D_{k}\right\} .\end{cases}
$$

Step 3. The energy of the test function. - We first note that the configuration $(\mathbf{z}, \mathbf{d})$ is s.t. $\hbar(\mathbf{z})>\frac{1}{2} \operatorname{dist}(\Lambda, \partial \Omega)$ and for $i \neq j$ we have $\frac{h_{\mathrm{ex}}^{-1}}{\left|z_{i}-z_{j}\right|} \rightarrow 0$, then we may apply Propositions 6.4, 6.5 and 6.7. We may also use Proposition 6.9. From these propositions we get

$$
\begin{aligned}
& \frac{1}{2} \int_{\Omega_{h_{\mathrm{ex}}-1}(\mathbf{z})}|\nabla v|^{2} \\
& =\pi d \ln h_{\mathrm{ex}}+W_{N_{0}}^{\operatorname{macro}}(\mathbf{p}, \mathbf{D})-\pi \sum_{\substack{k=1 \\
\text { s.t. } \bar{D}_{k} \geqslant 2}}^{N_{0}} \ln \left|z_{i}^{(k)}-z_{j}^{(k)}\right|+o(1) .
\end{aligned}
$$


For $k \in\left\{1, \ldots, N_{0}\right\}$ s.t. $D_{k} \geqslant 1$ and $i \in\left\{1, \ldots, D_{k}\right\}$ with (6.18), (6.19) and (6.20) we get:

$$
\begin{aligned}
& \frac{1}{2} \int_{B\left(z_{i}^{(k)}, h_{\mathrm{ex}}^{-1}\right) \backslash} \frac{{ }_{B\left(x_{i}^{(k)}, \lambda \delta^{2}\right)}}{} \alpha|\nabla v|^{2} \\
& =\pi\left|\ln \left(\lambda \delta h_{\mathrm{ex}}\right)\right|+b^{2} \pi|\ln (\delta)|+W^{\mathrm{micro}}\left(x_{0}\right)+o(1) .
\end{aligned}
$$

Note that $|\nabla v| \leqslant C \varepsilon^{-1}$. From Lemma IX.1 in [4] and (5.8), for $k \in\left\{1, \ldots, N_{0}\right\}$ s.t. $D_{k} \geqslant 1$ we have

$$
\frac{1}{2} \int_{B\left(x_{i}^{(k)}, \lambda \delta^{2}\right)} \alpha|\nabla v|^{2}+\frac{\alpha^{2}}{2 \varepsilon^{2}}\left(1-|v|^{2}\right)^{2}=b^{2} \pi \ln \left(b \lambda \delta^{2} / \varepsilon\right)+b^{2} \gamma+o(1)
$$

where $\gamma \in \mathbb{R}$ is a universal constant.

In conclusion, by combining (E.1), (E.2) and (E.3) (note $\lambda \delta h_{\mathrm{ex}} \rightarrow 0$ ):

$$
\begin{array}{r}
F(v) \leqslant d \pi\left[b^{2}|\ln \varepsilon|+\left(1-b^{2}\right)|\ln (\lambda \delta)|\right]+d\left[W^{\text {micro }}\left(x_{0}\right)+b^{2} \gamma+b^{2} \pi \ln b\right] \\
+W_{N_{0}}^{\operatorname{macro}}(\mathbf{p}, \mathbf{D})-\pi \sum_{\substack{k_{0} \\
\text { s.t. } \bar{D}_{k} \geqslant 2}} \sum_{i \neq j} \ln \left|z_{i}^{(k)}-z_{j}^{(k)}\right|+o(1) .
\end{array}
$$

Step 4. Definition of the magnetic potential and conclusion. - Let $A_{(\mathbf{z}, \mathbb{1})}$ be given by Definition 5.15 with $(\mathbf{a}, \mathbf{d})=(\mathbf{z}, \mathbb{1})$. It is clear that we have

$$
-\pi \sum_{\substack{k=1 \\ \text { s.t. } D_{k} \geqslant 2}}^{N_{0}} \sum_{i \neq j} \ln \left|z_{i}^{(k)}-z_{j}^{(k)}\right| \leqslant C|\ln \delta|
$$

where $C$ depends only on $d$ and $\Omega$.

Consequently, for $\varepsilon>0$ sufficiently small and $C_{0}>\pi d$ we have $F(v) \leqslant$ $C_{0}|\ln \varepsilon|$. Therefore, with Remark 5.16, the configuration $\left(v, A_{(\mathbf{z}, \mathbb{1})}\right) \in \mathscr{H}$ is s.t. $\mathcal{F}\left(v, A_{(\mathbf{z}, \mathbb{1})}\right) \leqslant \mathcal{F}(v, 0)+o(1) \leqslant C_{0}|\ln \varepsilon|^{2}+\mathcal{H}^{2}(\Omega) h_{\mathrm{ex}}^{2}$.

Using Proposition 5.7 and Lemma 5.14 we get

$$
\mathcal{F}\left(v, A_{(\mathbf{z}, \mathbb{1})}\right)=h_{\mathrm{ex}}^{2} \mathbf{J}_{\mathbf{0}}+2 \pi h_{\mathrm{ex}} \sum_{i=1}^{d} \xi_{0}\left(z_{i}\right)+F(v)+\widetilde{V}\left[\zeta_{(\mathbf{z}, \mathbb{1})}\right]+o(1)
$$

where $\zeta_{(\mathbf{z}, \mathbb{1})}$ is the unique solution of $(5.25)$ with $(\mathbf{a}, \mathbf{d})=(\mathbf{z}, \mathbb{1})$.

We now use Assertion 3 of Proposition 5.19 in order to get $\widetilde{V}\left[\zeta_{(\mathbf{z}, 1)}\right]=$ $\widetilde{V}\left[\zeta_{(\mathbf{p}, \mathbf{D})}\right]+o(1)$ and then

$$
\mathcal{F}\left(v, A_{(\mathbf{z}, \mathbb{1})}\right)=h_{\mathrm{ex}}^{2} \mathbf{J}_{\mathbf{0}}+2 \pi h_{\mathrm{ex}} \sum_{i=1}^{d} \xi_{0}\left(z_{i}\right)+F(v)+\widetilde{V}_{(\mathbf{z}, \mathbb{1})}\left[\zeta_{(\mathbf{p}, \mathbf{D})}\right]+o(1) .
$$


We claim that, from the choice of the points $z_{i}^{(k)}, \widetilde{z}_{i}^{(k)}$ we have $\xi_{0}\left(z_{i}^{(k)}\right)-$ $\xi_{0}\left(\widetilde{z}_{i}^{(k)}\right)=\mathcal{O}\left(\delta / \sqrt{h_{\mathrm{ex}}}\right)$. Thus with Proposition 6.10 we have

$$
\begin{aligned}
& -\pi \sum_{\substack{k=1 \\
\text { s.t. } D_{k} \geqslant 2}}^{N_{0}} \sum_{i \neq j} \ln \left|z_{i}^{(k)}-z_{j}^{(k)}\right|+2 \pi h_{\mathrm{ex}} \sum_{k=1}^{N_{0}} \sum_{i} \xi_{0}\left(z_{i}^{(k)}\right)-2 \pi d h_{\mathrm{ex}} \min _{\Omega} \xi_{0} \\
& =\sum_{\substack{k=1 \\
\text { s.t. } D_{k} \geqslant 1}}^{N_{0}}\left[-\pi \sum_{\substack{i, j \in\left\{1, \ldots, D_{k}\right\} \\
i \neq j}} \ln \left|\widetilde{z}_{i}^{(k)}-\widetilde{z}_{j}^{(k)}\right|+2 \pi h_{\mathrm{ex}} \sum_{i=1}^{D_{k}}\left[\xi_{0}\left(\widetilde{z}_{i}^{(k)}\right)-\min _{\Omega} \xi_{0}\right]+o(1)\right. \\
& =\sum_{\substack{k=1 \\
\text { s.t. } \bar{D}_{k} \geqslant 1}}^{N_{0}}\left[\frac{\pi}{2}\left(D_{k}^{2}-D_{k}\right) \ln \left(\frac{h_{\mathrm{ex}}}{D_{k}}\right)+C_{p_{k}, D_{k}}\right]+o(1) .
\end{aligned}
$$

We may now conclude:

$$
\begin{gathered}
\mathcal{F}(v, B)=h_{\mathrm{ex}}^{2} \mathbf{J}_{\mathbf{0}}+d M_{\Omega}\left[-h_{\mathrm{ex}}+H_{c_{1}}^{0}\right]+\frac{\pi}{2} \ln h_{\mathrm{ex}} \sum_{\substack{k=1 \\
\text { s.t. } \bar{D}_{k} \geqslant 1}}^{N_{0}}\left(D_{k}^{2}-D_{k}\right) \\
+\overline{\mathcal{W}}_{d}+\frac{\pi}{2} \sum_{\substack{k=1 \\
\text { s.t. } \bar{D}_{k} \geqslant 1}}^{N_{0}}\left(D_{k}-D_{k}^{2}\right) \ln D_{k}+o(1) .
\end{gathered}
$$

This estimate ends the proof of the proposition.

\section{Appendix F. Proof of Proposition 8.1}

Let $h_{\mathrm{ex}}$ and $\left(v_{\varepsilon}, A_{\varepsilon}\right)$ be as in Proposition 8.1. Note that we may assume that $A_{\varepsilon}=A_{v_{\varepsilon}}$ given by Lemma 5.8 and then $\left\|A_{\varepsilon}\right\|_{L^{\infty}(\Omega)}=\mathcal{O}\left(h_{\mathrm{ex}}\right)$. We drop the subscript $\varepsilon$. We first note that, by smoothness of $\Omega$, there is $t_{0}>0$, s.t. letting $\Omega_{t_{0}}:=\left\{x \in \mathbb{R}^{2} \mid \operatorname{dist}(x, \Omega)<t_{0}\right\}$, we may extend by reflexion $v \in H^{1}(\Omega, \mathbb{C})$ into $u \in H^{1}\left(\Omega_{t_{0}}, \mathbb{C}\right)$ letting $u=v$ in $\Omega$ and $u=v \circ \mathcal{S}_{\Omega}$ in $\Omega_{t_{0}} \backslash \bar{\Omega}$ where

$$
\begin{aligned}
\mathcal{S}_{\Omega}: \Omega_{t_{0}} \backslash \bar{\Omega} & \longrightarrow \Omega \\
x & \longmapsto \Pi(x)-\operatorname{dist}(x, \partial \Omega) \nu_{\Pi(x)} .
\end{aligned}
$$

Here $\Pi: \Omega_{t_{0}} \backslash \bar{\Omega} \rightarrow \partial \Omega$ is the orthogonal projection on $\partial \Omega$ and, for $\sigma \in \partial \Omega$, $\nu_{\sigma}$ is the normal outward at $\sigma$.

LemmA F.1. - Let $C_{0} \geqslant 1$ and let $\left\{\left(v_{\varepsilon}, A_{\varepsilon}\right) \mid 0<\varepsilon<1\right\}$ be a family in the Coulomb gauge of quasi-minimizers of $\mathcal{F}$ in $\mathscr{H}$ for an intensity of the applied field $h_{\mathrm{ex}}=h_{\mathrm{ex}}(\varepsilon) \geqslant 0$ s.t. $\|\nabla|v|\|_{L^{\infty}(\Omega)} \leqslant C_{0} \varepsilon^{-1}$. 
Under these hypotheses, for $\eta \in(0,1)$ there exists $\varepsilon_{\eta}, C_{\eta}>0$ (depending on $C_{0}$ ) s.t. for $0<\varepsilon<\varepsilon_{\eta}$, if $z \in \Omega$ is s.t.

$$
b^{2} \int_{B(z, \sqrt{\varepsilon} / 2)}|\nabla u|^{2}+\frac{b^{2}}{\varepsilon^{2}}\left(1-|u|^{2}\right)^{2} \leqslant \frac{C_{\eta}}{3}|\ln \varepsilon|
$$

with

$$
u= \begin{cases}v & \text { in } \Omega \\ v \circ \mathcal{S}_{\Omega} & \text { in } \Omega_{t_{0}} \backslash \bar{\Omega}\end{cases}
$$

then $|v(z)|>\eta$.

In order to prove Proposition 8.1 we need the following lemma.

LEMmA F.2. - There exists $\varepsilon_{\Omega}>0$ depending only on $\Omega$ s.t. for $0<\varepsilon<$ $\varepsilon_{\Omega}, z \in \Omega$ and $v \in H^{1}(\Omega, \mathbb{C})$, by defining $u$ as in Lemma F.1, the following inequality holds:

$$
\int_{B(z, \sqrt{\varepsilon} / 2)}|\nabla u|^{2}+\frac{b^{2}}{\varepsilon^{2}}\left(1-|u|^{2}\right)^{2} \leqslant 3 \int_{B(z, \sqrt{\varepsilon}) \cap \Omega}|\nabla v|^{2}+\frac{b^{2}}{\varepsilon^{2}}\left(1-|v|^{2}\right)^{2} .
$$

Proof of Lemma F.2. - In order to prove the lemma it suffices to check that by smoothness of $\Omega$ we have $\left\|\nabla\left(\mathcal{S}_{\Omega}^{-1}\right)\right\|_{L^{\infty}(\Omega)},\left\|\operatorname{Jac}\left(\mathcal{S}_{\Omega}^{-1}\right)\right\|_{L^{\infty}(\Omega)}=1+$ $o(1)$. We then immediately obtain

$$
\begin{aligned}
\int_{B(z, \sqrt{\varepsilon} / 2) \backslash \Omega}|\nabla u|^{2}+ & \frac{b^{2}}{\varepsilon^{2}}\left(1-|u|^{2}\right)^{2} \\
& \leqslant[1+o(1)] \int_{\mathcal{S}_{\Omega}[B(z, \sqrt{\varepsilon} / 2) \backslash \Omega]}|\nabla v|^{2}+\frac{b^{2}}{\varepsilon^{2}}\left(1-|v|^{2}\right)^{2} .
\end{aligned}
$$

On the other hand, if $x \in B(z, \sqrt{\varepsilon} / 2) \backslash \Omega$ then $\left|\mathcal{S}_{\Omega}(x)-z\right| \leqslant[1+o(1)] \sqrt{\varepsilon} / 2 \leqslant$ $\sqrt{\varepsilon}$ for sufficiently small $\varepsilon>0$ (depending only on $\Omega$ ). Then $\mathcal{S}_{\Omega}[B(z, \sqrt{\varepsilon} / 2) \backslash$ $\Omega] \subset B(z, \sqrt{\varepsilon}) \cap \Omega$. The lemma follows from the monotonicity of the integral.

By combining both lemmas we get Proposition 8.1.

Proof of Lemma F.1. - We argue by contradiction and we assume the existence of $\eta \in(0,1), \varepsilon=\varepsilon_{n} \downarrow 0$ s.t. for all $n \geqslant 1$ there are $(v, A)=$ $\left(v_{n}, A_{n}\right) \in \mathscr{H}, z=z_{n} \in \Omega$ and $h_{\mathrm{ex}}=h_{\mathrm{ex}}^{(n)} \geqslant 0$ s.t. $(v, A)$ is a quasiminimizers of $\mathcal{F}$ in $\mathscr{H}$ satisfying:

$$
\int_{B(z, \sqrt{\varepsilon} / 2)}|\nabla u|^{2}+\frac{b^{2}}{\varepsilon^{2}}\left(1-|u|^{2}\right)^{2} \leqslant \frac{|\ln \varepsilon|}{n}
$$

with

$$
u=u_{n}= \begin{cases}v & \text { in } \Omega \\ \varepsilon \circ \mathcal{S}_{\Omega} & \text { in } \Omega_{t_{0}} \backslash \bar{\Omega}\end{cases}
$$


and $|v(z)| \leqslant \eta$. Up to replacing $v$ by $\underline{v}$ we may assume $|v| \leqslant 1$ in $\Omega$.

We are going to prove that (F.1) implies

$$
\frac{1}{\varepsilon^{2}} \int_{B\left(z, \varepsilon^{3 / 4}\right) \cap \Omega}\left(1-|v|^{2}\right)^{2}=o(1) .
$$

On the other hand, $\|\nabla|v|\|_{L^{\infty}(\Omega)}=\mathcal{O}\left(\varepsilon^{-1}\right)$ and then, from an argument in [4, Theorem III.3], we will get, for sufficiently large $n,|v(z)|>\eta$. Clearly this contradiction will end the proof.

Since for $n \geqslant 1$ we have $\int_{\varepsilon^{3 / 4} / 2}^{\sqrt{\varepsilon} / 2} \frac{\mathrm{d} \rho}{\rho} \rho \int_{\partial B(z, \rho)}|\nabla u|^{2}+\frac{b^{2}}{\varepsilon^{2}}\left(1-|u|^{2}\right)^{2} \leqslant \frac{|\ln \varepsilon|}{n}$, there exists $\rho_{n} \in\left(\varepsilon^{3 / 4}, \sqrt{\varepsilon} / 2\right)$ s.t. $\rho_{n} \int_{\partial B\left(z, \rho_{n}\right)}|\nabla u|^{2}+\frac{b^{2}}{\varepsilon^{2}}\left(1-|u|^{2}\right)^{2} \leqslant \frac{4}{n}$. Then we get:

$$
\rho_{n} \int_{\partial B\left(z, \rho_{n}\right)}\left|\partial_{\tau} u\right|^{2}+\frac{b^{2}}{\varepsilon^{2}}\left(1-|u|^{2}\right)^{2} \leqslant \frac{4}{n} .
$$

We switch in polar coordinate and we denote $\widetilde{u}(\theta):=u\left(z+\rho_{n} \mathrm{e}^{\imath \theta}\right)$. Estimate (F.3) becomes

$$
\int_{0}^{2 \pi}\left|\partial_{\theta} \widetilde{u}\right|^{2}+\frac{b^{2} \rho_{n}^{2}}{\varepsilon^{2}}\left(1-|\widetilde{u}|^{2}\right)^{2} \leqslant \frac{4}{n} .
$$

On the one hand, $\left.\left|\partial_{\theta}\right| \widetilde{u}\right|^{2} \leqslant\left|\partial_{\theta} \widetilde{u}\right|^{2}$ and then $\int_{0}^{2 \pi}\left|\partial_{\theta}\right| \widetilde{u}|| \leqslant \frac{2 \sqrt{2 \pi}}{\sqrt{n}}$. Consequently in $[0,2 \pi]$ we get $\left(1-|\widetilde{u}|^{2}\right)^{2} \geqslant \max _{[0,2 \pi]}\left(1-|\widetilde{u}|^{2}\right)^{2}-\frac{2 \sqrt{2 \pi}}{\sqrt{n}}$. From (F.4) we deduce

$$
\frac{4 \varepsilon^{2}}{n b^{2} \rho_{n}^{2}} \geqslant \int_{0}^{2 \pi}\left(1-|\widetilde{u}|^{2}\right)^{2} \geqslant 2 \pi\left[\max _{[0,2 \pi]}\left(1-|\widetilde{u}|^{2}\right)^{2}-\frac{2 \sqrt{2 \pi}}{\sqrt{n}}\right]
$$

and thus for sufficiently large $n$ we get $0 \leqslant \max _{[0,2 \pi]}\left(1-|\widetilde{u}|^{2}\right)^{2} \leqslant \frac{100}{\sqrt{n}}$.

For a further use we define

$$
\begin{aligned}
\chi_{n}: B\left(z, \rho_{n}\right) & \longrightarrow[0,1] \\
z+\rho \mathrm{e}^{\imath \theta} & \longmapsto(|\widetilde{u}(\theta)|-1) \frac{\rho}{\rho_{n}}+1
\end{aligned}
$$

By direct calculations we have

$$
\int_{B\left(z, \rho_{n}\right)}\left|\nabla \chi_{n}\right|^{2}+\frac{1}{2 \varepsilon^{2}}\left(1-\chi_{n}^{2}\right)^{2}=\mathcal{O}\left(\frac{1}{n}\right) .
$$

On the other hand, for $n$ sufficiently large, $|u|^{2} \geqslant \frac{1}{2}$ in $\partial B\left(z, \rho_{n}\right)$. We thus may compute the degree of $u$ on $\partial B\left(z, \rho_{n}\right)$ and we find $\left|\operatorname{deg}_{\partial B\left(z, \rho_{n}\right)}(u)\right|=$ $\mathcal{O}\left(\frac{1}{n}\right)$ which implies, for sufficiently large $n, \operatorname{deg}_{\partial B\left(z, \rho_{n}\right)}(u)=0$. Consequently, we may write $u=|u| \mathrm{e}^{\imath \varphi}$ with $\varphi=\varphi_{n} \in H^{1}\left(\partial B\left(z, \rho_{n}\right), \mathbb{R}\right)$. Moreover, up to multiplying $u$ by a constant in $\mathbb{S}^{1}$, we may assume $\int_{\partial B\left(z, \rho_{n}\right)} \varphi=0$. 
We then consider $\widetilde{\varphi}:[0,2 \pi] \rightarrow \mathbb{R}$ defined by $\widetilde{\varphi}(\theta)=\varphi\left(z+\rho_{n} \mathrm{e}^{\imath \theta}\right)$, and thus

$$
\mathcal{O}\left(\frac{1}{n}\right)=\rho_{n} \int_{\partial B\left(z, \rho_{n}\right)}|\nabla \varphi|^{2} \geqslant \int_{0}^{2 \pi}\left|\partial_{\theta} \widetilde{\varphi}\right|^{2} .
$$

Since $\int_{0}^{2 \pi} \widetilde{\varphi}=0$, this estimate implies $\int_{0}^{2 \pi} \widetilde{\varphi}^{2}=\mathcal{O}\left(\frac{1}{n}\right)$.

Letting $\psi=\psi_{n}: B\left(z, \rho_{n}\right) \rightarrow \mathbb{R}, z+\rho \mathrm{e}^{\imath \theta} \mapsto \frac{\rho}{\rho_{n}} \widetilde{\varphi}(\theta)$, it is direct to check $\int_{B\left(z, \rho_{n}\right)}|\nabla \psi|^{2}=\mathcal{O}\left(\frac{1}{n}\right)$.

We are now in position to end the proof by considering $V=V_{n}=\chi_{n} \mathrm{e}^{\imath \psi} \in$ $H^{1}\left(B\left(z, \rho_{n}\right), \mathbb{C}\right)$ in order to have $V=v$ on $\partial B\left(z, \rho_{n}\right) \cap \Omega$,

$$
\frac{1}{2} \int_{\Omega \cap B\left(z, \rho_{n}\right)}|\nabla V|^{2}+\frac{1}{2 \varepsilon^{2}}\left(1-|V|^{2}\right)^{2}=\mathcal{O}\left(\frac{1}{n}\right) .
$$

and (with $\left.\|A\|_{L^{\infty}(\Omega)}=\mathcal{O}\left(h_{\mathrm{ex}}\right)\right)$

$$
\left|\int_{\Omega \cap B\left(z, \rho_{n}\right)} \alpha(V \wedge \nabla V) \cdot A\right| \leqslant C \frac{h_{\mathrm{ex}} \rho_{n}}{\sqrt{n}}=o(1) .
$$

Since $V=v$ on $\partial B\left(z, \rho_{n}\right) \cap \Omega$ we have

$$
w:=\left\{\begin{array}{ll}
v & \text { in } \Omega \backslash B\left(z, \rho_{n}\right) \\
V & \text { in } B\left(z, \rho_{n}\right) \cap \Omega
\end{array} \in H^{1}(\Omega, \mathbb{C}) .\right.
$$

Considering the comparison configuration $(w, A)$, from the quasi-minimality of $(v, A)$ and the above estimates we get

$$
\begin{aligned}
\int_{\Omega \cap B\left(z, \rho_{n}\right)}|\nabla v|^{2} & +\frac{1}{2 \varepsilon^{2}}\left(1-|v|^{2}\right)^{2} \\
& \leqslant b^{-4} \int_{\Omega \cap B\left(z, \rho_{n}\right)}|\nabla V|^{2}+\frac{1}{2 \varepsilon^{2}}\left(1-|V|^{2}\right)^{2}+o(1)=o(1) .
\end{aligned}
$$

Since $\rho_{n}>\varepsilon^{3 / 4}$ we get (F.2) and thus this estimate ends the proof.

\section{Appendix G. Proof of Proposition 8.3}

The proof of the proposition is an adaptation of the arguments presented in [2, Section V] and also used in [17, Proposition 3.2]. It is also inspired of the bad disk construction in [4]. Let $\mu, \lambda, \delta,(v, A)$ and $h_{\mathrm{ex}}$ be as in the proposition. 
Step 1. A first covering of $\{|v| \leqslant 1 / 2\}$. - For $0<\varepsilon<\varepsilon_{1 / 2}\left(\varepsilon_{1 / 2}>0\right.$ is given by Proposition 8.1 with $\eta=1 / 2$ ) we consider a covering of $\Omega$ by disks $\left\{B\left(x_{1}^{\varepsilon}, 4 \sqrt{\varepsilon}\right), \ldots, B\left(x_{N_{\varepsilon}}^{\varepsilon}, 4 \sqrt{\varepsilon}\right)\right\}$ s.t., for $i \neq j, B\left(x_{i}^{\varepsilon}, \sqrt{\varepsilon}\right) \cap B\left(x_{j}^{\varepsilon}, \sqrt{\varepsilon}\right)=\emptyset$ and $x_{i}^{\varepsilon} \in \Omega$.

For the simplicity of the presentation we omit the dependance in $\varepsilon$.

We say that $B\left(x_{i}, 4 \sqrt{\varepsilon}\right)$ is a bad disk if $\widetilde{E}_{\varepsilon}\left[v, B\left(x_{i}, 8 \sqrt{\varepsilon}\right) \cap \Omega\right]>C_{1 / 2}|\ln \varepsilon|$ where for a disk $B$ we denoted

$$
\widetilde{E}_{\varepsilon}(v, B \cap \Omega):=\int_{B \cap \Omega}|\nabla v|^{2}+\frac{1}{\varepsilon^{2}}\left(1-|v|^{2}\right)^{2}
$$

and $C_{1 / 2}>0$ is given by Proposition 8.1 with $\eta=1 / 2$. Let

$$
J^{\prime}=J_{\varepsilon}^{\prime}:=\left\{i \in\left\{1, \ldots, N_{\varepsilon}\right\} \mid B\left(x_{i}, 4 \sqrt{\varepsilon}\right) \text { is a bad disk }\right\} .
$$

We make two fundamental claims:

(1) There exists $M_{0} \geqslant 1$ (independent of $\varepsilon$ ) s.t. $\operatorname{Card}\left(J^{\prime}\right) \leqslant M_{0}$.

(2) If $B\left(x_{i}, 4 \sqrt{\varepsilon}\right)$ is not a bad disk then $|v| \geqslant 1 / 2$ in $B\left(x_{i}, 4 \sqrt{\varepsilon}\right)$.

The first claim is a direct consequence of $(5.15)$ and $B\left(x_{i}^{\varepsilon}, \sqrt{\varepsilon}\right) \cap B\left(x_{j}^{\varepsilon}, \sqrt{\varepsilon}\right)=$ $\emptyset$ for $i \neq j$.

The second claim is given by Proposition 8.1. Then $\bigcup_{i \in J^{\prime}} B\left(x_{i}, 4 \sqrt{\varepsilon}\right)$ is covering of $\{|v| \leqslant 1 / 2\}$ and $\operatorname{Card}\left(J^{\prime}\right) \leqslant M_{0}$.

Up to droping some disks, we may always assume that for $i \in J^{\prime}$ we have $B\left(x_{i}, 4 \sqrt{\varepsilon}\right) \cap\{|v| \leqslant 1 / 2\} \neq \emptyset$. Consequently using Corollary 8.2, for $i \in J^{\prime}$ and $0<\varepsilon<\min \left\{\varepsilon_{0}, \varepsilon_{1 / 2}\right\} \quad\left(\varepsilon_{0}\right.$ given by Corollary 8.2) we have $\operatorname{dist}\left(x_{i}, \Lambda\right)=\mathcal{O}\left(|\ln \varepsilon|^{-s_{0}}\right)$.

If $|v|>1 / 2$ in $\Omega$ then there is nothing to prove. We then assume $J^{\prime} \neq \emptyset$.

Step 2. Separation process. - We replace the above bad disks with disks having same centers and with a radius $\varepsilon^{\mu}$. Let $\varepsilon_{\mu}^{(1)}>0$ be s.t. $\min \left\{\varepsilon_{0}, \varepsilon_{1 / 2}\right\} \geqslant$ $\varepsilon_{\mu}^{(1)}$, for $0<\varepsilon<\varepsilon_{\mu}^{(1)}$ we have $4 \sqrt{\varepsilon}<\varepsilon^{\mu}$ and

$$
\max _{i \in J^{\prime}} \operatorname{dist}\left(B\left(x_{i}, \varepsilon^{\mu}\right), \Lambda\right) \leqslant \frac{1}{\ln |\ln \varepsilon|} .
$$

In particular $\bigcup_{i \in J^{\prime}} B\left(x_{i}, \varepsilon^{\mu}\right)$ is a covering of $\{|v| \leqslant 1 / 2\}$.

The goal of this step is to get a covering of $\{|v| \leqslant 1 / 2\}$ with disks $B\left(x_{i}, \varepsilon^{s}\right)$ where $i \in \widetilde{J}=\widetilde{J}_{\varepsilon} \subset J^{\prime}, s=s_{\varepsilon}=2^{-K} \mu, K=K_{\varepsilon} \in\left\{0, \ldots, M_{0}-1\right\}$ and s.t. for $i, j \in \widetilde{J}, i \neq j$, we have

$$
\left|x_{i}-x_{j}\right| \geqslant \varepsilon^{s / 2} .
$$


If $\operatorname{Card}\left(J^{\prime}\right)=1$ or (G.1) is satisfied with $s=\mu$ (i.e. $K=0$ ) then we let $\widetilde{J}=J^{\prime}$ and we obtained the desired result of this step. Otherwise, there are $i_{0}, j_{0} \in J^{\prime}$ (with $i_{0}<j_{0}$ ) s.t. $\left|x_{i_{0}}-x_{j_{0}}\right|<\varepsilon^{\mu / 2}$. In this case we let $J^{(1)}:=J^{\prime} \backslash\left\{i_{0}\right\}$ and we claim that $\operatorname{Card}\left(J^{(1)}\right)=\operatorname{Card}\left(J^{\prime}\right)-1$.

If $\operatorname{Card}\left(J^{(1)}\right)=1$ or $\operatorname{Card}\left(J^{(1)}\right)>1$ with (G.1) holds with $s=2^{-1} \mu$ (i.e. $K=1)$ for all $i, j \in J^{(1)}(i \neq j)$ then the goal of this step is done with $\widetilde{J}=J^{(1)}$ and $s=2^{-1} \mu$.

Otherwise, there exists $i_{0}, j_{0} \in J^{(1)}$ (with $\left.i_{0}<j_{0}\right)$ s.t. $\left|x_{i_{0}}-x_{j_{0}}\right|<\varepsilon^{s / 2}$. We then let $J^{(2)}:=J^{(1)} \backslash\left\{i_{0}\right\}$ and thus $\operatorname{Card}\left(J^{(2)}\right)=\operatorname{Card}\left(J^{(1)}\right)-1$.

By noting that $\operatorname{Card}\left(J^{\prime}\right) \leqslant M_{0}$, the above process stops after at most $M_{0}-1$ iteration. We thus get the existence of $K=K_{\varepsilon} \in\left\{0, \ldots, M_{0}-1\right\}$ and $\emptyset \neq J^{(K)}=J_{\varepsilon}^{(K)} \subset J^{\prime}$ s.t. $\operatorname{Card}\left(J^{(K)}\right)=1$ or $(\mathrm{G} .1)$ is satisfied with $s=s_{\varepsilon}=2^{-K} \mu$ and $i, j \in J^{(K)}(i \neq j)$.

We then denote $\widetilde{J}:=J^{(K)}, s=2^{-K} \mu$ and we fix $0<\varepsilon_{\mu}^{(2)} \leqslant \varepsilon_{\mu}^{(1)}$ s.t. for $0<\varepsilon<\varepsilon_{\mu}^{(2)}$ we have

$$
\max _{i \in \tilde{J}} \operatorname{dist}\left(B\left(x_{i}, \varepsilon^{s / 4}\right), \Lambda\right) \leqslant \frac{1}{\ln |\ln \varepsilon|}<10^{-1} \operatorname{dist}(\Lambda, \partial \Omega) .
$$

In particular $B\left(x_{i}, \varepsilon^{s / 4}\right) \subset \Omega$ for $i \in \widetilde{J}$.

Step 3. Definition of $r$. - With Corollary 5.2 in [5], for a.e. $t \in \operatorname{Im}(|v|)$ the set $V(t):=\{x \in \Omega|| v(x) \mid=t\}$ is a finite union of curve. Moreover if a such curve is included in $\Omega$ then it is a Jordan curve.

Following the same strategy as in [2, Lemma V.1], we have the existence of $t_{\varepsilon} \in\left[1-2|\ln \varepsilon|^{-2}, 1-|\ln \varepsilon|^{-2}\right]$ s.t. $V\left(t_{\varepsilon}\right)$ is a finite union of Jordan curves s.t.

$$
\mathcal{H}^{1}\left[V\left(t_{\varepsilon}\right)\right] \leqslant C \varepsilon|\ln \varepsilon|^{5} \text { with } C \text { is independent of } \varepsilon .
$$

We fix $0<\varepsilon_{\mu}^{(3)} \leqslant \varepsilon_{\mu}^{(2)}$ s.t. for $0<\varepsilon<\varepsilon_{\mu}^{(3)}$ we have $C \varepsilon|\ln \varepsilon|^{5} \leqslant 10^{-2} \varepsilon^{s}$.

We denote for $i \in \widetilde{J}$

$$
\mathcal{A}_{i}=\mathcal{A}_{i}^{\varepsilon}:=\left\{\rho \in\left[\varepsilon^{s}, \varepsilon^{2 s / 3}\right]|| v \mid \geqslant t_{\varepsilon} \text { on } \partial B\left(x_{i}, \rho\right)\right\} .
$$

From the continuity of $|v|$, it is clear that $\left[\varepsilon^{s}, \varepsilon^{2 s / 3}\right]=\mathcal{A}_{i} \cup \mathcal{B}_{i} \cup \mathcal{C}_{i}$ where

$$
\mathcal{B}_{i}=\mathcal{B}_{i}^{\varepsilon}:=\left\{\rho \in\left[\varepsilon^{s}, \varepsilon^{2 s / 3}\right] \mid \exists x \in \partial B\left(x_{i}, \rho\right) \text { s.t. }|v(x)|=t_{\varepsilon}\right\}
$$

and

$$
\mathcal{C}_{i}=\mathcal{C}_{i}^{\varepsilon}:=\left\{\rho \in\left[\varepsilon^{s}, \varepsilon^{2 s / 3}\right]|| v \mid<t_{\varepsilon} \text { on } \partial B\left(x_{i}, \rho\right)\right\} .
$$


We first claim that, since the function $\rho \mapsto \rho$ is increasing, we have

$$
\begin{aligned}
\mathcal{O}\left(\varepsilon^{2}|\ln \varepsilon|\right) & =\int_{\mathcal{C}_{i}} \mathrm{~d} \rho \int_{\partial B\left(x_{i}, \rho\right)}\left(1-|v|^{2}\right)^{2} \\
& \geqslant 2 \pi\left(1-t_{\varepsilon}^{2}\right)^{2} \int_{\mathcal{C}_{i}} \rho \mathrm{d} \rho \\
& \geqslant 2 \pi\left(1-t_{\varepsilon}^{2}\right)^{2} \int_{0}^{\mathcal{H}^{1}\left(\mathcal{C}_{i}\right)} \rho \mathrm{d} \rho=\pi\left(1-t_{\varepsilon}^{2}\right)^{2} \mathcal{H}^{1}\left(\mathcal{C}_{i}\right)^{2} .
\end{aligned}
$$

Then $\mathcal{H}^{1}\left(\mathcal{C}_{i}\right)=\mathcal{O}\left(\varepsilon|\ln \varepsilon|^{5 / 2}\right)$.

On the other hand one may prove that if $I$ is a connected components of $\mathcal{B}_{i}$, then there is $\rho_{1}, \rho_{2}$ s.t. $I=\left[\rho_{1}, \rho_{2}\right]$. Since straight lines are geodesics, we obviously get

$$
\mathcal{H}^{1}(I)=\rho_{2}-\rho_{1} \leqslant \mathcal{H}^{1}\left[V\left(t_{\varepsilon}\right) \cap \overline{B\left(x_{i}, \rho_{2}\right)} \backslash B\left(x_{i}, \rho_{1}\right)\right] .
$$

Moreover one may prove that if $\left[\rho_{1}, \rho_{2}\right]$ and $\left[\rho_{1}^{\prime}, \rho_{2}^{\prime}\right]$ are distinct connected component of $\mathcal{B}_{i}$ and if $\Gamma$ is a connected component of $V\left(t_{\varepsilon}\right)$ s.t. $\Gamma \cap \overline{B\left(x_{i}, \rho_{2}\right)} \backslash$ $B\left(x_{i}, \rho_{1}\right) \neq \emptyset$ then $\Gamma \cap \overline{B\left(x_{i}, \rho_{2}^{\prime}\right)} \backslash B\left(x_{i}, \rho_{1}^{\prime}\right)=\emptyset$ (here we used (G.2)). One may conclude: $\mathcal{H}^{1}\left(\mathcal{B}_{i}\right) \leqslant \mathcal{H}^{1}\left(V\left(t_{\varepsilon}\right)\right) \leqslant C \varepsilon|\ln \varepsilon|^{5}$.

Consequently

$$
\begin{aligned}
\mathcal{H}^{1}\left(\mathcal{A}_{i}\right) & \geqslant \mathcal{H}^{1}\left(\left[\varepsilon^{s}, \varepsilon^{2 s / 3}\right]\right)-\mathcal{H}^{1}\left(\mathcal{B}_{i}\right)-\mathcal{H}^{1}\left(\mathcal{C}_{i}\right) \\
& \geqslant \varepsilon^{2 s / 3}-\varepsilon^{s}-\mathcal{H}^{1}\left(V\left(t_{\varepsilon}\right)\right)-\mathcal{O}\left(\varepsilon|\ln \varepsilon|^{5 / 2}\right) .
\end{aligned}
$$

Fix $0<\varepsilon_{\mu}^{(4)} \leqslant \varepsilon_{\mu}^{(3)}$ s.t. for $0<\varepsilon<\varepsilon_{\mu}^{(4)}$ we have $\mathcal{H}^{1}\left(\mathcal{A}_{i}\right) \geqslant \varepsilon^{2 s / 3}-\varepsilon^{s}-\sqrt{\varepsilon}$.

Define

$$
\mathcal{A}=\mathcal{A}_{\mu, \varepsilon}:=\cap_{i \in \tilde{J}} \mathcal{A}_{i} .
$$

It is clear that $\mathcal{H}^{1}(\mathcal{A}) \geqslant \varepsilon^{2 s / 3}-\varepsilon^{s}-M_{0} \sqrt{\varepsilon}$

Since $\rho \mapsto 1 / \rho$ is decreasing we have

$$
\begin{aligned}
\mathcal{O}(|\ln \varepsilon|) & \geqslant \int_{\mathcal{A}} \frac{\mathrm{d} \rho}{\rho} \sum_{i \in \tilde{J}} \rho \int_{\partial B\left(x_{i}, \rho\right)}|\nabla v|^{2}+\frac{1}{\varepsilon^{2}}\left(1-|v|^{2}\right)^{2} \\
& \geqslant \int_{\varepsilon^{2 s / 3}-\mathcal{H}^{1}(\mathcal{A})}^{\varepsilon^{2 s / 3}} \frac{\mathrm{d} \rho}{\rho} \times \inf _{\rho \in \mathcal{A}} \sum_{i \in \tilde{J}} \rho \int_{\partial B\left(x_{i}, \rho\right)}|\nabla v|^{2}+\frac{1}{\varepsilon^{2}}\left(1-|v|^{2}\right)^{2} .
\end{aligned}
$$

Consequently, there exist $r=r_{\mu, \varepsilon} \in \mathcal{A}, C_{\mu} \geqslant 1\left(C_{\mu}\right.$ is independent of $\left.\varepsilon\right)$ and $0<\varepsilon_{\mu}^{(5)} \leqslant \varepsilon_{\mu}^{(4)}$ s.t. for $0<\varepsilon<\varepsilon_{\mu}^{(5)}$ we have

$$
\sum_{i \in \tilde{J}} r \int_{\partial B\left(x_{i}, r\right)}|\nabla v|^{2}+\frac{1}{\varepsilon^{2}}\left(1-|v|^{2}\right)^{2} \leqslant C_{\mu} .
$$

We finally let $J_{\mu}:=\widetilde{J}$, with (G.1) and (G.5) the result is proved. 


\section{Appendix H. Proof of Proposition 8.10}

The proof is an adaptation of the proof of (VI.21) in [2].

Let $\widetilde{\alpha}=\widetilde{\alpha}_{n} \in L^{\infty}\left(\Omega,\left[\beta^{2}, 1\right]\right),(\mathbf{z}, \mathbf{d})=(\mathbf{z}, \mathbf{d})^{(n)} \in\left(\Omega^{N}\right)^{*} \times \mathbb{Z}^{N}$ and $u=$ $u_{n} \in H^{1}(\Omega, \mathbb{C})$ be as in the proposition.

We first claim that up to considering $\underline{u}$ instead of $u$ we may assume $|u| \leqslant 1$ in $\Omega$. Note also that if $\int_{\Omega_{\tilde{r}}}|\nabla u|^{2} \geqslant \beta^{-2} \int_{\Omega_{\tilde{r}}}\left|\nabla w_{\star}^{(\mathbf{z}, \mathbf{d})}\right|^{2}$, then there is nothing to prove. We thus may assume

$$
\int_{\Omega_{\tilde{r}}}|\nabla u|^{2}<\beta^{-2} \int_{\Omega_{\tilde{r}}}\left|\nabla w_{\star}^{(\mathbf{z}, \mathbf{d})}\right|^{2} .
$$

Let $w:=u /|u| \in H^{1}\left(\Omega_{\tilde{r}}, \mathbb{S}^{1}\right)$. From Lemma I.1 in [4] we have $w \wedge \nabla w=$ $\nabla^{\perp} \Phi_{\star}^{(\mathbf{z}, \mathbf{d})}+\nabla H$ with $H=H_{\varepsilon} \in H^{1}\left(\Omega_{\tilde{r}}, \mathbb{R}\right)$ and

$$
\int_{\Omega_{\tilde{r}}}|\nabla H|^{2} \leqslant\left(\beta^{-1}+1\right)^{2} \int_{\Omega_{\tilde{r}}}\left|\nabla \Phi_{\star}^{(\mathbf{z}, \mathbf{d})}\right|^{2} .
$$

Let $\Phi_{\tilde{r}}$ be the unique solution of (D.1). We have $\int_{\Omega_{\tilde{r}}} \nabla H \cdot \nabla^{\perp} \Phi_{\tilde{r}}=0$. Then letting $\rho=|u|$ :

$$
\begin{aligned}
\int_{\Omega_{\tilde{r}}} \widetilde{\alpha} \rho^{2} & \nabla H \cdot \nabla^{\perp} \Phi_{\star}^{(\mathbf{z}, \mathbf{d})} \\
& =\int_{\Omega_{\tilde{r}}}\left(\widetilde{\alpha} \rho^{2}-1\right) \nabla H \cdot \nabla^{\perp} \Phi_{\star}^{(\mathbf{z}, \mathbf{d})}+\int_{\Omega_{\tilde{r}}} \nabla H \cdot\left(\nabla^{\perp} \Phi_{\star}^{(\mathbf{z}, \mathbf{d})}-\nabla^{\perp} \Phi_{\tilde{r}}\right) .
\end{aligned}
$$

But, from (D.12), there exists $C \geqslant 1$ s.t.

$$
\left|\int_{\Omega_{\tilde{r}}} \nabla H \cdot\left(\nabla^{\perp} \Phi_{\star}^{(\mathbf{z}, \mathbf{d})}-\nabla^{\perp} \Phi_{\tilde{r}}\right)\right| \leqslant C\|\nabla H\|_{L^{2}\left(\Omega_{\tilde{r}}\right)} \sqrt{X}
$$

where $X$ is defined in (6.8).

Consequently, letting $\widetilde{C}:=4 C^{2} / \beta^{2}$ we get

$$
\begin{aligned}
2 \int_{\Omega_{\tilde{r}}} \nabla H \cdot \nabla^{\perp} \Phi_{\star}^{(\mathbf{z}, \mathbf{d})} & +\int_{\Omega_{\tilde{r}}} \widetilde{\alpha} \rho^{2}|\nabla H|^{2} \\
& =2 \int_{\Omega_{\tilde{r}}} \nabla H \cdot\left(\nabla^{\perp} \Phi_{\star}^{(\mathbf{z}, \mathbf{d})}-\nabla^{\perp} \Phi_{\tilde{r}}\right)+\int_{\Omega_{\tilde{r}}} \widetilde{\alpha} \rho^{2}|\nabla H|^{2} \\
& \geqslant\|\nabla H\|_{L^{2}\left(\Omega_{\tilde{r}}\right)}\left(\frac{\beta^{2}}{4}\|\nabla H\|_{L^{2}\left(\Omega_{\tilde{r}}\right)}-2 C \sqrt{X}\right) \\
& \geqslant-\widetilde{C} X .
\end{aligned}
$$


Therefore

$$
\begin{aligned}
\int_{\Omega_{\tilde{r}}} \widetilde{\alpha} \rho^{2}|\nabla w|^{2} \geqslant \int_{\Omega_{\tilde{r}}}\left|\nabla \Phi_{\star}^{(\mathbf{z}, \mathbf{d})}\right|^{2} & -\int_{\Omega_{\tilde{r}}}\left(1-\widetilde{\alpha} \rho^{2}\right)\left|\nabla \Phi_{\star}^{(\mathbf{z}, \mathbf{d})}\right|^{2} \\
& -2 \int_{\Omega_{\tilde{r}}}\left(1-\widetilde{\alpha} \rho^{2}\right)|\nabla H|\left|\nabla \Phi_{\star}^{(\mathbf{z}, \mathbf{d})}\right|-\mathcal{O}(X) .
\end{aligned}
$$

On the other hand, using (6.7) and Corollary 6.6, we get

$$
\begin{aligned}
\left.\left|\int_{\Omega_{\tilde{r}}}\left(1-\widetilde{\alpha} \rho^{2}\right)\right| \nabla \Phi_{\star}^{(\mathbf{z}, \mathbf{d})}\right|^{2} \mid & \leqslant\left.\left|\int_{\Omega_{\tilde{r}}}\left(1-\rho^{2}\right)\right| \nabla \Phi_{\star}^{(\mathbf{z}, \mathbf{d})}\right|^{2}|+| \int_{\Omega_{\tilde{r}}}(1-\widetilde{\alpha})\left|\nabla \Phi_{\star}^{(\mathbf{z}, \mathbf{d})}\right|^{2} \mid \\
& \leqslant\left\|\nabla \Phi_{\star}^{(\mathbf{z}, \mathbf{d})}\right\|_{L^{\infty}\left(\Omega_{\tilde{r}}\right)}\left\|\nabla \Phi_{\star}^{(\mathbf{z}, \mathbf{d})}\right\|_{L^{2}\left(\Omega_{\tilde{r}}\right)}(K+L)
\end{aligned}
$$

and with (H.1):

$$
\begin{aligned}
& \left|\int_{\Omega_{\tilde{r}}}\left(1-\widetilde{\alpha} \rho^{2}\right)\right| \nabla H|| \nabla \Phi_{\star}^{(\mathbf{z}, \mathbf{d})}|| \\
& \quad \leqslant\left|\int_{\Omega_{\tilde{r}}}\left(1-\rho^{2}\right)\right| \nabla H|| \nabla \Phi_{\star}^{(\mathbf{z}, \mathbf{d})}||+\left|\int_{\Omega_{\tilde{r}}}(1-\widetilde{\alpha})\right| \nabla H|| \nabla \Phi_{\star}^{(\mathbf{z}, \mathbf{d})}|| \\
& \leqslant\left\|\nabla \Phi_{\star}^{(\mathbf{z}, \mathbf{d})}\right\|_{L^{\infty}\left(\Omega_{\tilde{r})}\right)}\left\|\nabla \Phi_{\star}^{(\mathbf{z}, \mathbf{d})}\right\|_{L^{2}\left(\Omega_{\tilde{r}}\right)}(K+L)\left(2 \beta^{-1}+1\right) .
\end{aligned}
$$

The proposition is thus proved.

\section{Appendix I. Proof of Proposition 9.6}

We prove the first assertion and we assume $\operatorname{Card}\left(J_{\mu}\right) \geqslant 2$. We let $\chi_{1}:=$ $2 h_{\mathrm{ex}}^{-1} \ln h_{\mathrm{ex}}, \chi_{2}:=2 h_{\mathrm{ex}}^{-1 / 2} \ln h_{\mathrm{ex}}$ and $\Omega_{\chi_{2}}=\Omega \backslash \bigcup_{p \in \Lambda} \overline{B\left(p, \chi_{2}\right)}$.

In order to get sufficiently sharp estimates to prove the proposition, we decompose $\Omega_{r}$ in several subdomains. To this aim, we distinguish two cases for $p \in \Lambda$ : either $\operatorname{Card}\left(J_{p}^{(y)}\right) \geqslant 2$ or $\operatorname{Card}\left(J_{p}^{(y)}\right) \in\{0,1\}$ where $J_{p}^{(y)}:=\{k \in$ $\left.J^{(y)} \mid y_{k} \in B\left(p, \chi_{2}\right)\right\}$ (the $y_{k}$ 's are introduced in Definition 9.2).

If $p \in \Lambda$ is s.t. $\operatorname{Card}\left(J_{p}^{(y)}\right) \geqslant 2$, then with Lemma 8.9 (with $P=17$ and $\left.\eta=\chi_{1} / 2\right)$, there are $\kappa_{p}=\kappa_{p, \varepsilon} \in\left\{17^{0}, \ldots, 17^{N_{0}-1}\right\}$ and $\widetilde{J}_{p}^{(y)} \subset J_{p}^{(y)}$ s.t.

$$
\left\{\begin{array}{l}
\bigcup_{k \in J_{p}^{(y)}} B\left(y_{k}, \chi_{1} / 2\right) \subset \bigcup_{k \in \tilde{J}_{p}^{(y)}} B\left(y_{k}, \kappa_{p} \chi_{1} / 2\right) \\
\left|y_{k}-y_{l}\right| \geqslant 8 \kappa_{p} \chi_{1} \text { for } k, l \in \widetilde{J}_{p}^{(y)}, k \neq l .
\end{array}\right.
$$

We then let $\mathcal{D}_{p}:=B\left(p, \chi_{2}\right) \backslash \bigcup_{k \in \tilde{J}_{p}^{(y)}} \overline{B\left(y_{k}, \kappa_{p} \chi_{1}\right)}$ and, for $k \in \widetilde{J}_{p}^{(y)}$, we write $\underline{d}_{k}:=\operatorname{deg}_{\partial B\left(y_{k}, \kappa_{p} \chi_{1}\right)}(v)$. We denote also $D_{p}:=\sum_{k \in \tilde{J}_{p}^{(y)}} \underline{d}_{k}$ 
If $p \in \Lambda$ is s.t. $J_{p}^{(y)}=\{k\}$, then we let $\mathcal{D}_{p}=B\left(p, \chi_{2}\right) \backslash \overline{B\left(y_{k}, \kappa \delta\right)}$ with $\kappa$ given by Definition 9.2. We let also $D_{p}:=\underline{d}_{k}:=\operatorname{deg}_{\partial B\left(y_{k}, \kappa \delta\right)}(v)$.

Recall that we denoted (see Definition 9.2), for $k \in J^{(y)}, \widetilde{d}_{k}:=$ $\operatorname{deg}_{\partial B\left(y_{k}, \kappa \delta\right)}(v)$. Consequently, if $J_{p}^{(y)}=\{k\}$, then $D_{p}=\underline{d}_{k}=\widetilde{d}_{k}$.

If $J_{p}^{(y)}=\emptyset$ then we denote $D_{p}=0$ and $\mathcal{D}_{p}=B\left(p, \chi_{2}\right)$.

The heart of the proof consists in proving that $\underline{d}_{k}=1$ for all $k$. Indeed, we know that if $i \in J_{\mu}$ then $\operatorname{deg}_{\partial B\left(z_{i}, r\right)}(v)=1$. Consequently $\underline{d}_{k}$ is the number of points $z_{i}$ contained in a disk of radius at least $\chi_{1}$.

We let:

- $\mathcal{R}:=\bigcup_{k \in J^{(y)}} B\left(y_{k}, \kappa \delta\right) \backslash \bigcup_{i \in J_{\mu}} \overline{B\left(z_{i}, r\right)}, \kappa$ given in Definition 9.2.

- For $p \in \Lambda$ s.t. $\operatorname{Card}\left(J_{p}^{(y)}\right) \geqslant 2$ and for $k \in \widetilde{J}_{p}^{(y)}$ we denote

$$
\mathcal{Q}_{k, p}:=B\left(y_{k}, \kappa_{p} \chi_{1}\right) \backslash \bigcup_{\substack{l \in J^{(y)} \\ y_{l} \in B\left(y_{k}, \kappa_{p} \chi_{1}\right)}} \overline{B\left(y_{l}, \kappa \delta\right)} .
$$

Moreover, by construction, we have (for sufficiently small $\varepsilon$ )

$$
\bigcup_{\substack{l \in J^{(y)} \\ y_{l} \in B\left(y_{k}, \kappa_{p} \chi_{1}\right)}} B\left(y_{l}, \kappa \delta\right) \subset \bigcup_{\substack{l \in J^{(y)} \\ y_{l} \in B\left(y_{k}, \kappa_{p} \chi_{1}\right)}} B\left(y_{l}, \chi_{1} / 2\right) \subset B\left(y_{k}, \kappa_{p} \chi_{1} / 2\right) .
$$

Thus

$$
\begin{aligned}
\frac{1}{2} \int_{\Omega_{r}} \alpha|\nabla v|^{2} \geqslant & \frac{1}{2} \int_{\mathcal{R}} \alpha|\nabla v|^{2}+\sum_{p \in \Lambda} \frac{1}{2} \int_{\mathcal{D}_{p}} \alpha|\nabla v|^{2} \\
& +\sum_{\substack{p \in \Lambda \\
\operatorname{Card}\left(J_{p}^{(y)}\right) \geqslant 2}} \sum_{k \in \tilde{J}_{p}^{(y)}} \frac{1}{2} \int_{\mathcal{Q}_{k, p}} \alpha|\nabla v|^{2}+\frac{1}{2} \int_{\Omega_{\chi_{2}}} \alpha|\nabla v|^{2} .
\end{aligned}
$$

From (9.11) and (9.12) we have

$$
\frac{1}{2} \int_{\mathcal{R}} \alpha|\nabla v|^{2} \geqslant d \pi\left[b^{2}|\ln r|+\left(1-b^{2}\right)|\ln \lambda|-b^{2}|\ln \delta|\right]+\mathcal{O}(1) .
$$

If $J_{p}^{(y)}=\{k\}$, then with Corollary 8.8(1) we get

$$
\frac{1}{2} \int_{\mathcal{D}_{p}} \alpha|\nabla v|^{2} \geqslant \pi \underline{d}_{k}^{2} \ln \left(\frac{\chi_{2}}{\delta}\right)+\mathcal{O}(1) .
$$

And if $\operatorname{Card}\left(J_{p}^{(y)}\right) \geqslant 2$, still with Corollary 8.8(1):

$$
\frac{1}{2} \int_{\mathcal{D}_{p}} \alpha|\nabla v|^{2} \geqslant \pi \sum_{k \in \tilde{J}_{p}^{(y)}} \underline{d}_{k}^{2} \ln \left(\frac{\chi_{2}}{\chi_{1}}\right)+\mathcal{O}(1) .
$$


We continue by dealing with the case $\operatorname{Card}\left(J_{p}^{(y)}\right) \geqslant 2$. From Corollary 8.8(1) applied in $\mathcal{Q}_{k, p}$ for $k \in \widetilde{J}_{p}^{(y)}$ (with (I.1)) we get

$$
\sum_{k \in \tilde{J}_{p}^{(y)}} \frac{1}{2} \int_{\mathcal{Q}_{k, p}} \alpha|\nabla v|^{2} \geqslant \pi \sum_{k \in \tilde{J}_{p}^{(y)}} \sum_{\substack{l \in J^{(y)} \\ y_{l} \in B\left(y_{k}, \kappa_{p} \chi_{1}\right)}} \widetilde{d}_{l}^{2} \ln \left(\frac{\chi_{1}}{\delta}\right)+\mathcal{O}(1)
$$

In order to end the proof, using Propositions 6.4, 6.5 and 8.10, we get

$$
\frac{1}{2} \int_{\Omega_{\chi_{2}}} \alpha|\nabla v|^{2} \geqslant \pi \sum_{p \in \Lambda} D_{p}^{2}\left|\ln \chi_{2}\right|+\mathcal{O}(1) .
$$

We let

$$
\Delta:=\sum_{\substack{p \in \Lambda \text { s.t. } \\ \operatorname{Card}\left(J_{p}^{(y)}\right) \geqslant 2}} \sum_{k \in \tilde{J}_{p}^{(y)}} \underline{d}_{k}^{2}+\sum_{\substack{p \in \Lambda \text { s.t. } \\ J_{p}^{(y)}=\{k\}}} \underline{d}_{k}^{2} \text { and } \widetilde{\Delta}:=\sum_{k \in J^{(y)}} \widetilde{d}_{k}^{2} .
$$

From (I.2), (I.3), (I.4), (I.5), (I.6) and (I.7) we get

$$
\begin{aligned}
& \frac{1}{2} \int_{\Omega_{r}} \alpha|\nabla v|^{2} \\
& \geqslant \mathcal{O}(1)+d \pi\left[b^{2}|\ln r|+\left(1-b^{2}\right)|\ln \lambda|-b^{2}|\ln \delta|\right]+\pi \sum_{\substack{p \in \Lambda \text { s.t. } \\
J_{p}^{(y)}=\{k\}}} \underline{d}_{k}^{2} \ln \left(\frac{\chi_{2}}{\delta}\right) \\
& +\pi \sum_{\substack{p \in \Lambda \\
\operatorname{Card}\left(J_{p}^{(y)}\right) \geqslant 2}}\left[\sum_{k \in \tilde{J}_{p}^{(y)}} \underline{d}_{k}^{2} \ln \left(\frac{\chi_{2}}{\chi_{1}}\right)+\sum_{\substack{l \in J^{(y)} \\
y l \in B\left(p, \chi_{2}+\lambda \delta\right)}} \widetilde{d}_{l}^{2} \ln \left(\frac{\chi_{1}}{\delta}\right)\right]+\pi \sum_{p \in \Lambda} D_{p}^{2}\left|\ln \chi_{2}\right| \\
& \geqslant d \pi\left[b^{2}|\ln r|+\left(1-b^{2}\right)|\ln (\lambda \delta)|\right]+\pi\left|\ln \chi_{2}\right|\left(\sum_{p \in \Lambda} D_{p}^{2}-\Delta\right)+\pi|\ln \delta|(\widetilde{\Delta}-d) \\
& +\pi\left|\ln \chi_{1}\right| \sum_{\substack{p \in \Lambda \\
\operatorname{Card}\left(J_{p}^{(y)}\right) \geqslant 2}}\left[\sum_{\substack{l \in \tilde{J}_{p}^{(y)} \\
\underline{d}_{k}^{2}-\widetilde{d}_{l}^{(y)}}} \widetilde{d}_{l}^{2}\right]+\mathcal{O}(1) .
\end{aligned}
$$

Since $\underline{d}_{k}, \widetilde{d}_{l} \geqslant 1$ for all $k, l$, from Lemma $9.5(1)$ we have $\sum_{p \in \Lambda} D_{p}^{2} \geqslant \Delta \geqslant$ $\widetilde{\Delta} \geqslant d$ and moreover

$$
\Delta=d \Longleftrightarrow\left(\underline{d}_{k}=1 \text { for all } k\right)
$$

and

$$
\widetilde{\Delta}=d \Longleftrightarrow\left(\widetilde{d}_{l}=1 \text { for all } l\right) .
$$


On the other hand since for $p \in \Lambda$ s.t. $J_{p}^{(y)}=\{k\}$ we have $\underline{d}_{k}=\widetilde{d}_{k}$, we get

$$
\Delta-\widetilde{\Delta}=\sum_{\substack{p \in \Lambda \\ \operatorname{Card}\left(J_{p}^{(y)}\right) \geqslant 2}}\left[\sum_{k \in \tilde{J}_{p}^{(y)}} \underline{d}_{k}^{2}-\sum_{\substack{l \in J^{(y)} \\ y_{l} \in B\left(p, \chi_{2}+\lambda \delta\right)}} \widetilde{d}_{l}^{2}\right] .
$$

Then (9.6) gives

$\frac{\mathscr{L}_{1}(d)}{\pi} \ln h_{\mathrm{ex}} \geqslant\left(\sum_{p \in \Lambda} D_{p}^{2}-\Delta\right)\left|\ln \chi_{2}\right|+(\widetilde{\Delta}-d)|\ln \delta|+(\Delta-\widetilde{\Delta})\left|\ln \chi_{1}\right|+\mathcal{O}(1)$.

Since $\left|\ln \chi_{1}\right|=\ln \left(h_{\mathrm{ex}}\right)+\mathcal{O}\left[\ln \left(\ln h_{\mathrm{ex}}\right)\right]$ and $\left|\ln \chi_{2}\right|=\ln \sqrt{h_{\mathrm{ex}}}+\mathcal{O}\left[\ln \left(\ln h_{\mathrm{ex}}\right)\right]$ we obtain

$$
\begin{aligned}
\left(\frac{\mathscr{L}_{1}(d)}{\pi}\right. & \left.+\frac{d-\sum_{p \in \Lambda} D_{p}^{2}}{2}\right) \ln h_{\mathrm{ex}} \\
& \geqslant(\Delta-\widetilde{\Delta}) \ln \sqrt{h_{\mathrm{ex}}}+(\widetilde{\Delta}-d)\left|\ln \left(\delta \sqrt{h_{\mathrm{ex}}}\right)\right|+\mathcal{O}\left[\ln \left(\ln h_{\mathrm{ex}}\right)\right] .
\end{aligned}
$$

From Lemma $9.5(2)$ and the definition of $\mathscr{L}_{1}(d)$ (see Lemma 7.1), we have

$$
\frac{\mathscr{L}_{1}(d)}{\pi}+\frac{d-\sum_{p \in \Lambda} D_{p}^{2}}{2} \leqslant 0 .
$$

Using (I.9) in (I.8), (1.4), $\widetilde{\Delta}-d \geqslant 0$ and $\Delta-\widetilde{\Delta} \geqslant 0$ we get $\widetilde{\Delta}-d=$ $\Delta-\widetilde{\Delta}=0$ and then $\Delta=d$, i.e. $\underline{d}_{k}=1$ for all $k$.

On the other hand, with the help of (I.8) we may write

$$
0 \geqslant\left(\frac{\mathscr{L}_{1}(d)}{\pi}+\frac{d-\sum_{p \in \Lambda} D_{p}^{2}}{2}\right) \ln h_{\mathrm{ex}} \geqslant \mathcal{O}\left[\ln \left(\ln h_{\mathrm{ex}}\right)\right]
$$

We may thus deduce $\frac{\mathscr{L}_{1}(d)}{\pi}+\frac{d-\sum_{p \in \Lambda} D_{p}^{2}}{2}=0$ and then, with Lemma $9.5(2)$, for $p \in \Lambda$ we have $D_{p} \in\left\{\left\lfloor d / N_{0}\right\rfloor ;\left\lceil d / N_{0}\right\rceil\right\}$.

\section{Bibliography}

[1] A. Aftalion, É. Sandier \& S. Serfaty, "Pinning phenomena in the GinzburgLandau model of superconductivity", J. Math. Pures Appl. 80 (2001), no. 3, p. 339372.

[2] L. Almeida \& F. Bethuel, "Topological methods for the Ginzburg-Landau equations", J. Math. Pures Appl. 77 (1998), no. 1, p. 1-49. 
[3] F. Bethuel, H. Brézis \& F. Hélein, "Asymptotics for the minimization of a Ginzburg-Landau functional", Calc. Var. Partial Differ. Equ. 1 (1993), no. 2, p. 123148.

[4] - Ginzburg-Landau Vortices, Progress in Nonlinear Differential Equations and their Applications, vol. 13, Birkhäuser, 1994.

[5] J. Bourgain, M. V. Korobkov \& J. Kristensen, "On the Morse-Sard property and level sets of Sobolev and BV functions", Rev. Mat. Iberoam. 1 (2013), no. 29, p. 1-23.

[6] M. Dos Santos, "The Ginzburg-Landau functional with a discontinuous and rapidly oscillating pinning term. Part II: the non-zero degree case", Indiana Univ. Math. J. 62 (2013), no. 2, p. 551-641.

[7] — "Microscopic renormalized energy for a pinned Ginzburg-Landau functional", Calc. Var. Partial Differ. Equ. 53 (2015), no. 1-2, p. 65-89.

[8] —, "Explicit expression of the microscopic renormalized energy for a pinned Ginzburg-Landau functional", https://hal.archives-ouvertes.fr/ hal-01684216v2, 2018.

[9] M. Dos Santos \& O. Misiats, "Ginzburg-Landau model with small pinning domains", Netw. Heterog. Media 6 (2011), no. 4, p. 715-753.

[10] D. Gilbarg \& N. S. Trudinger, Elliptic partial differential equations of second order, Classics in Mathematics, Springer, 2015.

[11] A. Kachmar, "Magnetic vortices for a Ginzburg-Landau type energy with discontinuous constraint", ESAIM, Control Optim. Calc. Var. 16 (2009), no. 3, p. 545-580.

[12] L. Lassoued \& P. Mironescu, "Ginzburg-Landau type energy with discontinuous constraint", J. Anal. Math. 77 (1999), p. 1-26.

[13] C. Lefter \& V. RĂdulescu, "Minimization problems and corresponding renormalized energies", Differ. Integral Equ. 9 (1996), no. 5, p. 903-917.

[14] J. Rubinstein, "On the equilibrium position of Ginzburg-Landau vortices", $Z$. Angew. Math. Phys. 46 (1995), no. 5, p. 739-751.

[15] É. Sandier \& S. Serfaty, "Ginzburg-Landau minimizers near the first critical field have bounded vorticity", Calc. Var. Partial Differ. Equ. 17 (2003), no. 1, p. 17-28.

[16] - Vortices in the magnetic Ginzburg-Landau model, Progress in Nonlinear Differential Equations and their Applications, vol. 70, Birkhäuser, 2007.

[17] S. SERfATY, "Local minimizers for the Ginzburg-Landau energy near critical magnetic field: Part I", Commun. Contemp. Math. 1 (1999), no. 2, p. 213-254. 